\title{
Optical Analysis of Synaptic Plasticity Underlying Associative Learning in Drosophila melanogaster
}

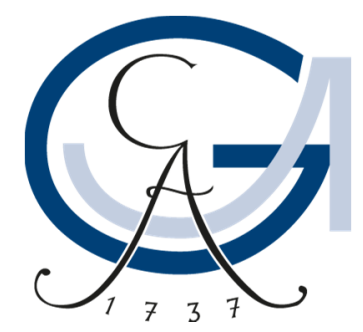

Dissertation

for the award of the degree

"Doctor rerum naturalium"

of the Georg-August-Universität Göttingen

within the doctoral program (Sensory and M otor Neuroscience)

of the Göttingen Graduate School for Neurosciences, Biophysics, and M olecular Biosciences (GGNB)

\section{submitted by \\ Florian Bilz}

from

Wismar, Germany

Göttingen 2018 


\section{PhD Committee Members:}

Prof. Dr. André Fiala (Supervisor, Reviewer)

Dept. of Molecular Neurobiology of Behavior, Georg-August-University, Göttingen

Prof. Dr. Ralf Heinrich (Reviewer)

Dept. of Cellular Neurobiology, Georg-August-University, Göttingen

Camin Dean, PhD

Dept. of Trans-synaptic Signaling, European Neuroscience Institute, Göttingen

\section{Members of the Examination Board:}

Prof. Dr. André Fiala (Supervisor, Reviewer)

Dept. of Molecular Neurobiology of Behavior, Georg-August-University, Göttingen

Prof. Dr. Ralf Heinrich (Reviewer)

Dept. of Cellular Neurobiology, Georg-August-University, Göttingen

Camin Dean, PhD

Dept. of Trans-synaptic Signaling, European Neuroscience Institute Göttingen (ENI), Göttingen

\section{Additional Members of the Examination Board:}

Dr. Marion Silies

AG Visual Processing, European Neuroscience Institute Göttingen (ENI), Göttingen

Prof. Dr. Tim Gollisch

Department of Opthalmology, School of M edicine, Universitätsmedizin Göttingen, Göttingen

Prof. Dr. Nils Brose

Abtl. Molekulare Neurobiologie, Max-Planck-Institut für Experimentelle M edizin, Göttingen

Thesis submission: July 31st, 2018

Thesis defense: September 20th, 2018 


\section{Declaration of academic honesty:}

I hereby declare that the doctoral thesis entitled "Optical Analysis of Synaptic Plasticity Underlying Associative Learning in Drosophila melanogaster" has been written independently and with no other sources and aids than quoted within the text, references and acknowledgements.

Florian Bilz

Göttingen, July 31st, 2018 

***D iese A rbeit widme ich meiner $0 \mathrm{ma}$ J ohanna $\mathrm{E}$ lisabeth $B \quad$ ilz, die für immer in meinem $H$ erzen weiter existieren wirdl*** $* 22.09 .1927-\dagger 25.07 .2014$ 



\section{Table of Contents}

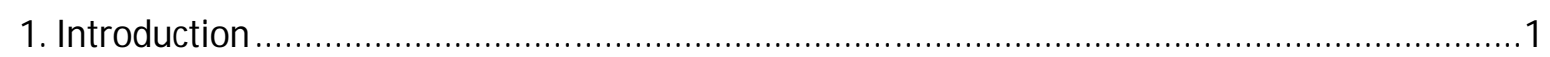

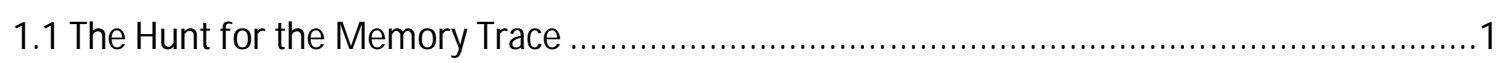

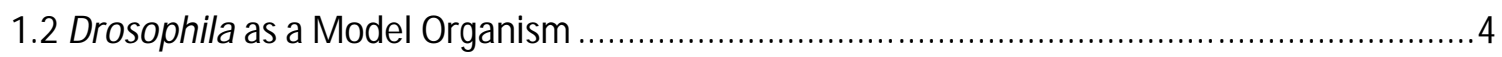

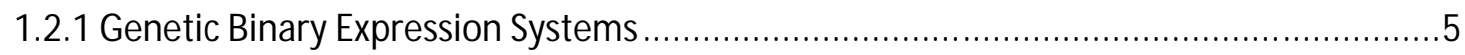

1.2.2 M osaic Analysis with a Repressible Cell M arker ....................................................

1.2.3 Identification and Functional Characterization of Neurons and Circuits in Drosophila ....8

1.3 The Olfactory System of Drosophila.....................................................................10

1.4 The M ushroom Bodies - Key Structures in Associative Learning and M emory .....................12

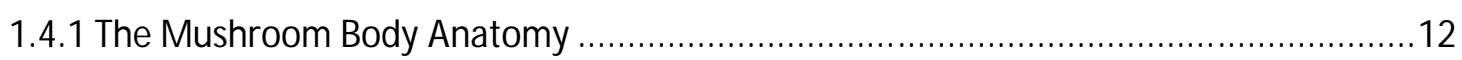

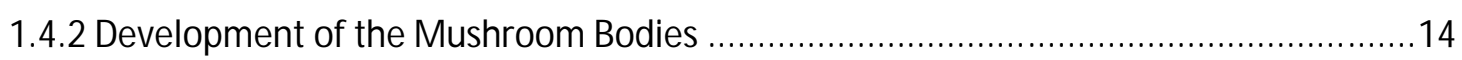

1.4.3 Connectivities of the M ushroom Bodies..................................................................15

1.4.4 Physiology of KCs

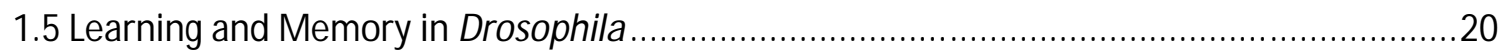

1.5.1 Synaptic Plasticity - The Key to Memory Formation ............................................2

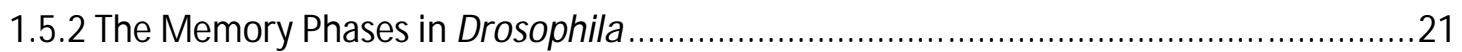

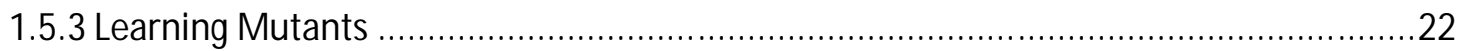

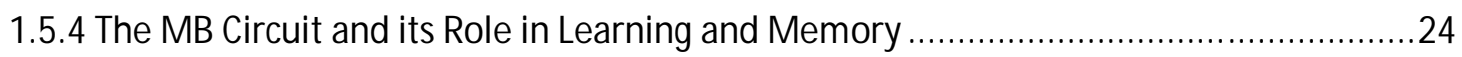



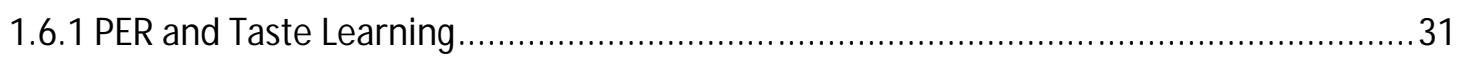

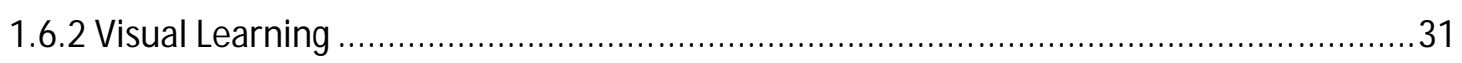

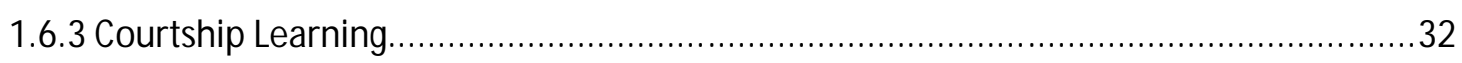



1.7 Scope of the Study

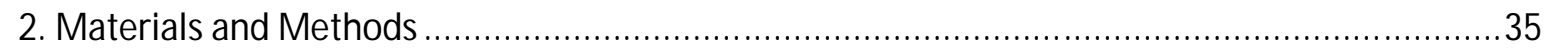

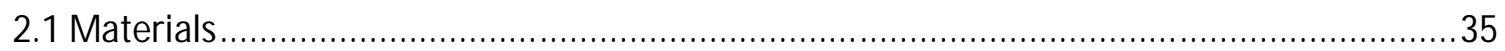

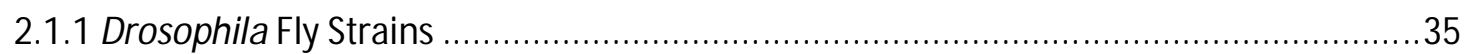

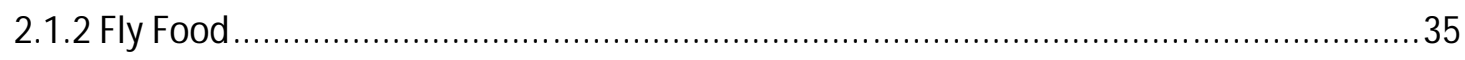

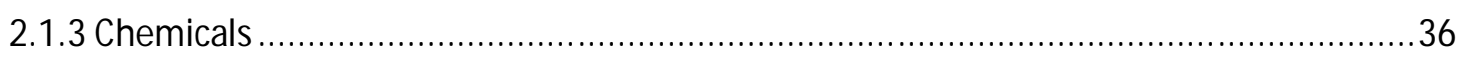

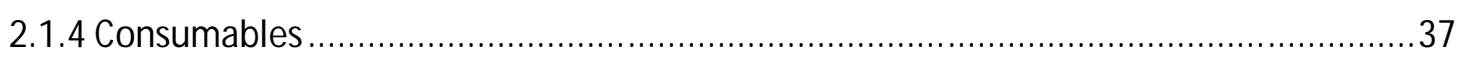

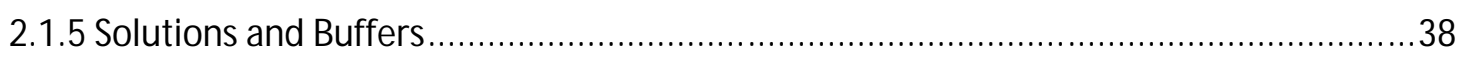

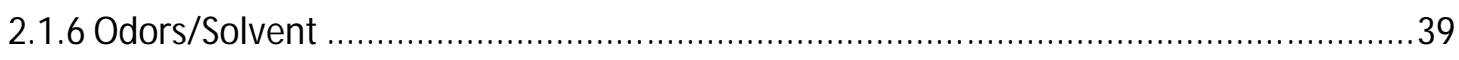

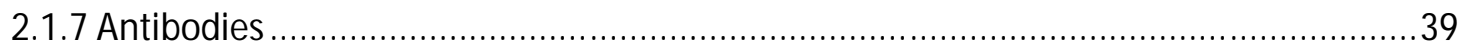




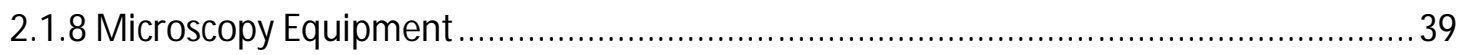

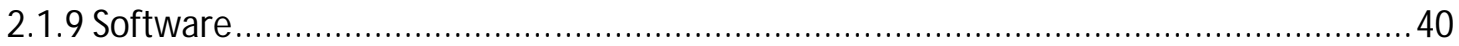

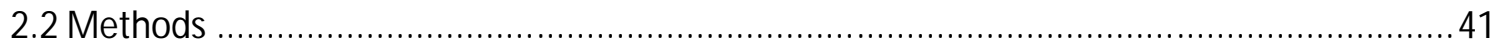

2.2.1 Generation of Fly Strains for M ARCM Experiments ........................................... 41

2.2.2 Generation of Single Cell Clones with the MARCM -Technique ................................. 44

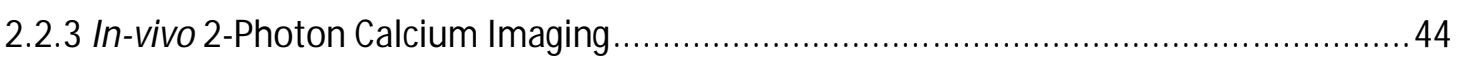

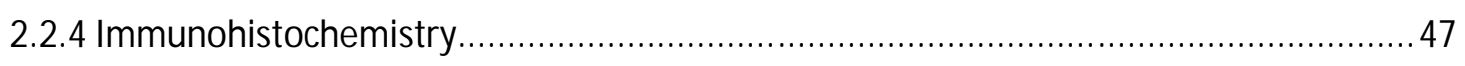

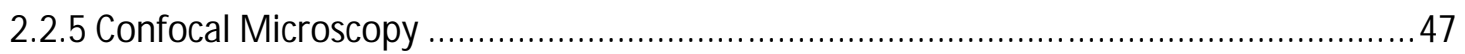

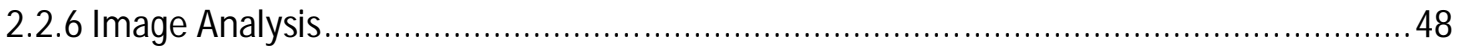

2.2.7 Bouton Similarity Analysis - Activity Corrected Correlation......................................49

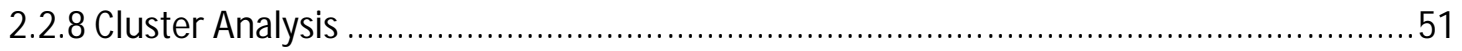

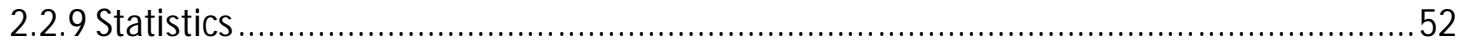

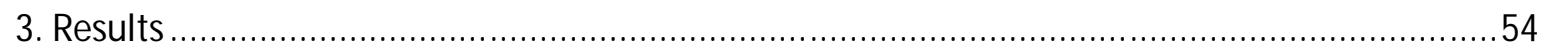

3.1 Establishing Single Kenyon Cell Calcium Imaging......................................................5

3.1.1 Generation of Single $\gamma$-KCS with the M ARCM -Technique ......................................5

3.1.2 Boutons in $\mathrm{y}$-KC Axons are Rich in the Pre-Synaptic Protein Bruchpilot .......................57

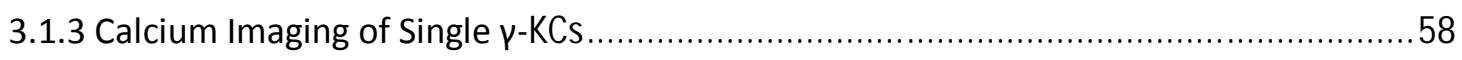

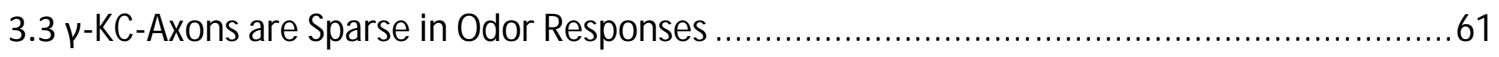

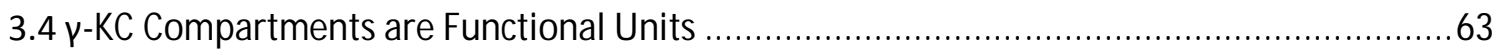

3.4.1 $\gamma$-KCs Exhibit Highly Individual Response Profiles ................................................. 63

3.4.2 A novel Similarity Analysis Demonstrates the Functional Independence of $\gamma$-Lobe

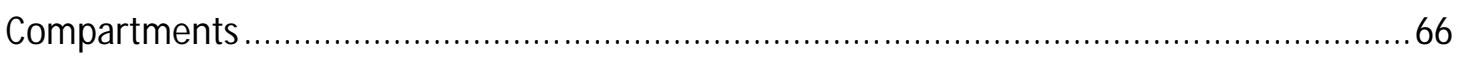

3.5 Olfactory Associative Training Changes the Odor Code ................................................69

3.6 $\mathrm{Y}$-KC Boutons Form Clusters That Are Changed in the Course of Associative Training.......... 76

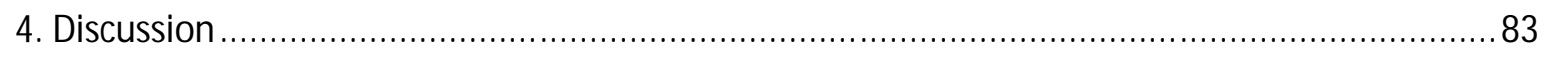

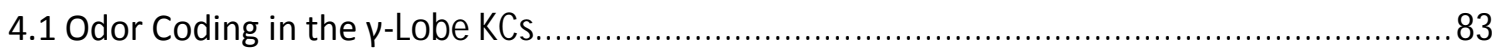

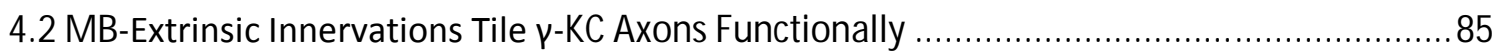

4.3 Aversive Associative Learning Changes the Reinforced Odor Representation at the Synaptic

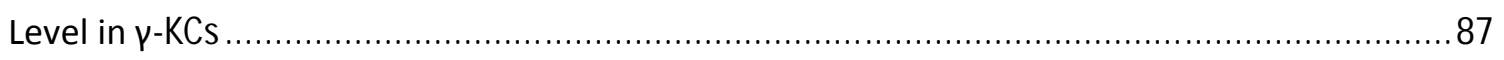

4.4 What Can Drosophila Tell Us About the Engram? ......................................................... 91

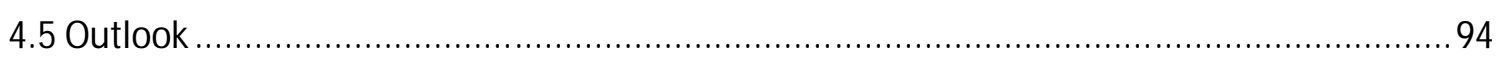

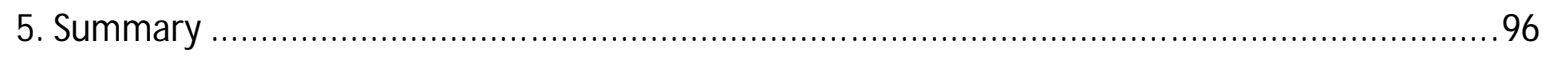

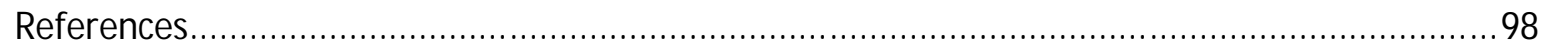

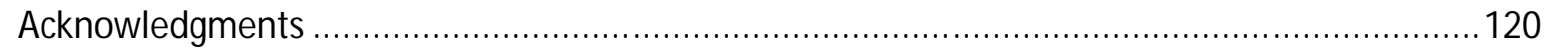

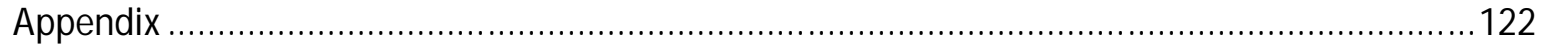


A1 Abbreviations..

Curriculum vitae ... 

Introduction

\section{Introduction}

\subsection{The Hunt for the Memory Trace}

The fascination of how the brain works has always thrilled humans. The vast amount of stimuli and information the brain can process and store, consciously or unconsciously, is nearly intangible. Therefore, it is even more astounding that a given set of cells contacting in uncountable connections creates such an entity capable of storing and recalling this information. In an environment that is selecting for the strongest and fittest individual or group of individuals it is crucial to adapt to changes, memorize information and consequences, and to later recall this information. The storage of such information or memory is, to our best knowledge, localized to the brain. But what is this memory exactly? How and where is it stored specifically? And finally, how can it be retrieved?

Since more than 100 years many milestones were reached on the search for the memory trace and brought scientists closer to unveiling the secrets behind memory formation (summarized in: Josselyn et al., 2015, 2017; Poo et al., 2016). A memory is based on the entirety of physiological changes left in the nervous system induced by external stimulation. Those physiological changes that are required and sufficient to form, store, and retrieve the memory are called memory traces. Memory traces are defined by several criteria (Gerber et al., 2004; M artin et al., 2000; Thompson, 2005): First of all, if a memory is formed it has to be detectable in the form of synaptic plasticity in some neuronal substrate. If this synaptic plasticity is driven by external stimuli, the artificial activation of these inputs should elicit the same memory-induced behavioral output in this particular neuronal substrate. This also implies that the disruption of these learning-relevant inputs to this neuronal substrate should block memory formation. If a neuronal substrate shows synaptic plasticity it should ultimately alter the output of these neurons and therefore change the input to downstream neurons. Therefore, the block of the output of this neuronal substrate should disrupt memory as well. To form a memory is only one side of the coin. As the learned experience is important to change the behavior appropriately in the future, recall of the memory is essential. Therefore, the block of a potential memory-relevant neuronal substrate during memory retrieval should impair the memory-relevant behavioral output. The difficulties in finding memory traces lie in the network architecture of brains. A brain consists of many thousands to many millions of neurons, each of which possess large numbers of synapses. Therefore, the chance to detect memory traces is very low and the task very challenging. Furthermore, there is 


\section{Introduction}

not just one centralized brain structure storing memory traces but a great distribution across neurons and neuronal populations (Davis, 2011; Liu et al., 2006; Thompson and Steinmetz, 2009; Thum et al., 2007). Moreover, many brain structures encode information as sparsely activated neuron ensembles (Honegger et al., 2011; Perez-Orive, 2002; Stettler and Axel, 2009; Stopfer et al., 2003; Turner et al., 2008) that decrease the probability of detecting relevant neurons responding to memory-relevant stimuli.

The principle of a memory trace was first postulated by the German zoologist Richard Semon who called the memory trace the "engram" (Semon, 1904). He hypothesized that certain brain cells triggered by external stimulation can store a memory (engram) and that the reactivation of these cells can recall the memory. But how is this process accomplished by the nervous system? One possible answer was given already in 1894 by Santiago Ramón y Cajal and his famous anatomical drawings of nerve cells who proposed that the outgrowth or modification of existing synaptic structures are the mechanisms to store memory traces (Cajal, 1894). Based on Cajals assumptions, Donald Hebb postulated in 1949 his famous theory about how neurons can strengthen their connections to each other and laid the foundation for the investigation of synaptic plasticity (Hebb, 1949). He suggested that repeated accompanied firing of a neuron and its downstream neuron develops or strengthens a synaptic knob and that this strengthening is the neural translation of association. From these theories one of the best-known sentences in neuroscience emerged: "What fires together, wires together". At the same time, Jerzy Konorski independently proposed a similar mechanism for neural plasticity that is dependent on timed stimulation of two neurons, forming as a result excitatory synapses (Konorski, 1948). Both proposals were confirmed by experiments showing e.g., a long term potentiation of the postsynaptic hippocampal dentate area neurons in anesthetized rabbits (Bliss and Lømo, 1973), short term and long term sensitization in the marine snail Aplysia (Castellucci et al., 1989; Schwartz et al., 1971) and short term plasticity in the VUM mx1 neuron in honeybees (Hammer, 1993). Furthermore, it was shown that the potentiation of synaptic connections is only formed if the first neuron is firing shortly before the second neuron - a mechanism which is known as spike timing-dependent plasticity (see review: Caporale and Dan, 2008). These experiments made Hebb one of the discoverers of synaptic plasticity that opened up completely new fields in neuroscience. However, the rule of "Hebbian plasticity" appears to be too simple when taking into account the huge research field of associative learning. In associative learning, an additional neuronal layer modulates synaptic strength by conveying aversive or appetitive stimuli to the preand post-synaptic neuron via neuromodulators e.g., dopamine in both vertebrates (Janak and Tye, 2015; Kandel et al., 2014) as well as invertebrates (Kandel et al., 2014; Perisse et al., 2013a), 
extending the Hebb-model by the neuromodulatory system. This further indicates that synaptic plasticity not only occurs in the post-synapse but also at the pre-synapse.

Forming and memorizing associations in nature is a crucial ability for the survival of an individual as it allows for adaption to an ever-changing environment and avoidance of harmful or seeking beneficial encounters e.g., predators or toxic substances and food sources or potential mates, respectively. To investigate the mechanism underlying associative learning and memory, experiments utilizing associative conditioning paradigms were developed. Associative conditioning comprises two major forms: classical conditioning and operant conditioning. The basic principle underlying classical conditioning is the association of a stimulus with an involuntary behavioral response. The term operant conditioning describes the association of a self-exerted behavior and its consequence facilitating or diminishing this behavior. In 1898 the first experiments on operant conditioning were described by Edward Lee Thorndike under a different term ("law of effects" or instrumental conditioning) where he observed the learning curve of cats that had to escape a contraption-box by pulling e.g., a rope (Thorndike, 1898). Almost 40 years later famous operant conditioning experiments were conducted by Burrhus Frederic Skinner who designed the so-called "Skinner box" in which a lever is installed that releases food to a tray when pushed appropriately (Skinner, 1938). A rat can be placed into this box and pushes the lever while initially exploring the box. After repeated encounters with the lever the rat learned the consequence of pushing the lever (that is, food delivery) and intentionally pushes the lever to get food. The first prominent experiments on classical conditioning were performed by Ivan Petrovich Pavlov in 1906 who experimented with dogs salivating when food was presented (Pavlov, 1906). He demonstrated that if a natural or unconditioned stimulus (US) e.g., food is presented to a dog, it starts salivating - the unconditioned response (UR). If a neutral stimulus (NS) e.g., a tone is repeatedly presented preceding the US (e.g., food), this stimulus becomes the conditioned stimulus (CS) as its sole presentation can elicit the former UR that therefore becomes the conditioned response (CR).

Classical conditioning has been investigated in many model organisms, as well as humans. One well known experiment in humans was the fear conditioning experiment with the infant Albert in which an initially neutrally perceived rat (NS) was paired with loud, fear evoking (UR) noises (US) eliciting fear responses (CR) when the rat (CS) was later presented alone (Watson and Rayner, 1920). In other experiments with humans, Grant and Adams (1944) conditioned the eyelid reflex (UR) to air puffs (US) with light pulses (CS) that later elicited the eyelid reflex (CR) alone (Grant and Adams, 1944). Similar experiments were carried out by Gormenzano and colleagues who used albino rabbits and conditioned the eyelid reflex to air puffs of the outer lid and the nictitating membrane to a tone (Gormenzano et al., 1962; Schneiderman et al., 1961). In a more 
Introduction

invasive approach, Patterson and colleagues anesthetized cats and conditioned the hind limb flexion reflex by electrically stimulating the skin (US) and the leg nerves (CS) - a method called spinal reflex conditioning (Patterson et al., 1973). An also very famous procedure of classical conditioning is the fear conditioning in animals - including humans - where aversive stimuli (US) are presented together with the CS eliciting fear responses like freezing, startle, flight, etc. involving the amygdala as a key associative center (see reviews: Maren, 2001; Rescorla, 1967, 1968). However, not only vertebrates were shown to be capable of associative learning; also invertebrate model organisms could be used for classical conditioning. One example is the conditioning of the gill withdrawal reflex of the giant marine snail Aplysia californica that showed increased withdrawal responses after a classical conditioning protocol (Carew et al., 1983, 1981; Hawkins, 1984). In these preparations, it could also be shown that the cellular mechanism underlying classical conditioning involves the CAMP/PKA pathway (see. 1.5; and reviewed by Hawkins, 1984; Kahsai and Zars, 2011; Kandel, 2012) that was first described for non-associative sensitization in Aplysia (Byrne and Kandel, 1996; Castellucci et al., 1982; Cedar et al., 1972 and see review: Kandel, 2001). Besides cellular mechanisms, the readout of learning performance was also investigated in invertebrates. A prominent example of invertebrate classical conditioning is the honey bee Apis mellifera that can associate olfactory or visual cues with sucrose or electric shock stimulation, where the proboscis extension reflex (PER) and the sting extension reflex (SER), respectively, are robust measures for the learning performance (Kuwabara, 1957; Takeda, 1961; Vergoz et al., 2007; and see also reviews: Menzel, 2012; M enzel and Müller, 1996). However, the technically most versatile invertebrate model organism even until today is Drosophila melanogaster, which changed the learning field dramatically due to its genetic accessibility that allows for the precise study, monitoring, and manipulation of learning and memory.

\subsection{Drosophila as a Model Organism}

Learning and memory is studied in model organisms as these provide physical and genetic accessibility that humans can't provide. The basic principles of learning and memory remain comparable across animals ranging from vertebrate (e.g., cats, rabbits, rats, and mice) to invertebrate species (e.g., Aplysia, crayfish, honeybees, and fruit flies; reviewed by Kandel et al., 2014). However, the advantage of invertebrate model organisms is their often lower neuronal complexity. Especially, the fruit fly Drosophila melanogaster moved strongly into focus over the past decades. In the search of memory traces it is necessary to investigate the different neuronal substrates that are involved in the process of learning and memory. Vertebrate model organisms, 
with their complex fore-, mid-, and hindbrain structures and millions of neurons, are very difficult to access and comprise very complicated network structures. On the contrary, Drosophila comprises a relatively low number of neurons but is still complex enough to perform well in numerous learning and behavioral tasks (Heisenberg, 2003; Hige, 2018; Pitman et al., 2009; Wolf et al., 1998) and allows for the search of the distributed memory traces. Due to Drosophila's genetic accessibility and the availability of techniques allowing for the manipulation and monitoring of neuronal circuits, neurons, and even synapses (Venken et al., 2011), it is a wellsuited model organism to study associative learning and memory. Therefore, it was used in this study and the following sections and chapters will focus on techniques used to study learning and memory in Drosophila as well as the knowledge that was gathered about learning and memory in Drosophila so far.

\subsubsection{Genetic Binary Expression Systems}

A first step into elucidating the neuronal mechanisms of learning and memory requires the investigation of potential neuronal substrates. One of the great advantages of Drosophila is its elaborated genetic techniques to express transgenes that can monitor or manipulate specifically targetable neurons. A commonly used technique - also utilized in this study - is the binary expression of the yeast GAL4/UAS system (Fig. 1.1). Binary expression systems are genetic tools that rely on the specific interaction of transcriptional proteins and their specific matching DNA binding sites (reviewed Ptashne, 1988). One of the most commonly used tools in Drosophila genetics is the GAL4/UAS-system (Brand and Perrimon, 1993; Fischer et al., 1988) that was derived from yeast. Due to its exclusive expression in only yeast it does not interfere with any Drosophila intrinsic gene expression and can be used with high certainty. Furthermore, it allows for temporal and/or spatial restriction of gene expression in a specific set of cell populations. In this system, the GAL4 protein works as a transcription factor that binds to a specific DNAsequence that is called UAS (upstream activating sequence, Fig. $1.1 \mathrm{a}$ and b). GAL4 expression can be targeted to specific cell types via the cloning of a cell-specific promoter sequence to GAL4 or via using a P-transposase gene vector with GAL4 to randomly integrate into the genome (Fig. 1.1 a), also called enhancer trap (Brand and Perrimon, 1993). Any protein sequence e.g., fluorescent proteins can be cloned into the vector behind the UAS-sequence (Fig. $1.1 \mathrm{a}$ ). Only if the expression of GAL4 and the UAS-reporter sequence come together in a cell the reporter gene can be expressed, allowing for precise cell targeting (Fig. 1.1 b).

Nowadays, there are thousands of GAL4-lines available, but often the GAL4 insertion is not $100 \%$ specific to a desired cell type, as the promoter can be also active in other cells. This is of particular 
concern if a cell type is supposed to be physiologically manipulated. An observed effect can't be unambiguously assigned to the manipulated cells as it could also arise from the desired manipulation of other cells expressed in this GAL4 driver. To overcome this problem, the splitGAL4 system was developed in which two parts of the GAL4 protein can be independently expressed with different promoter sequences only leading to a functioning GAL4 protein if both promoters are active in the same cell providing higher specificity (Luan et al., 2006). The introduction of the repressor GAL80 that binds to GAL4 preventing its transcription initiating function (Fig. $1.1 \mathrm{~b}$ and c) further improved the genetic labelling as the function of GAL4 can be temporally controlled via e.g., a heat shock (Lee and Luo, 1999; Ma and Ptashne, 1987; M cGuire, 2003). Besides the GAL4/UAS-system other binary systems such as LexA/lexAop (Lai and Lee, 2006) and QF/QUAS (Potter et al., 2010) were developed and can be used in combination with each other to allow for non-overlapping cell manipulation and monitoring, respectively.

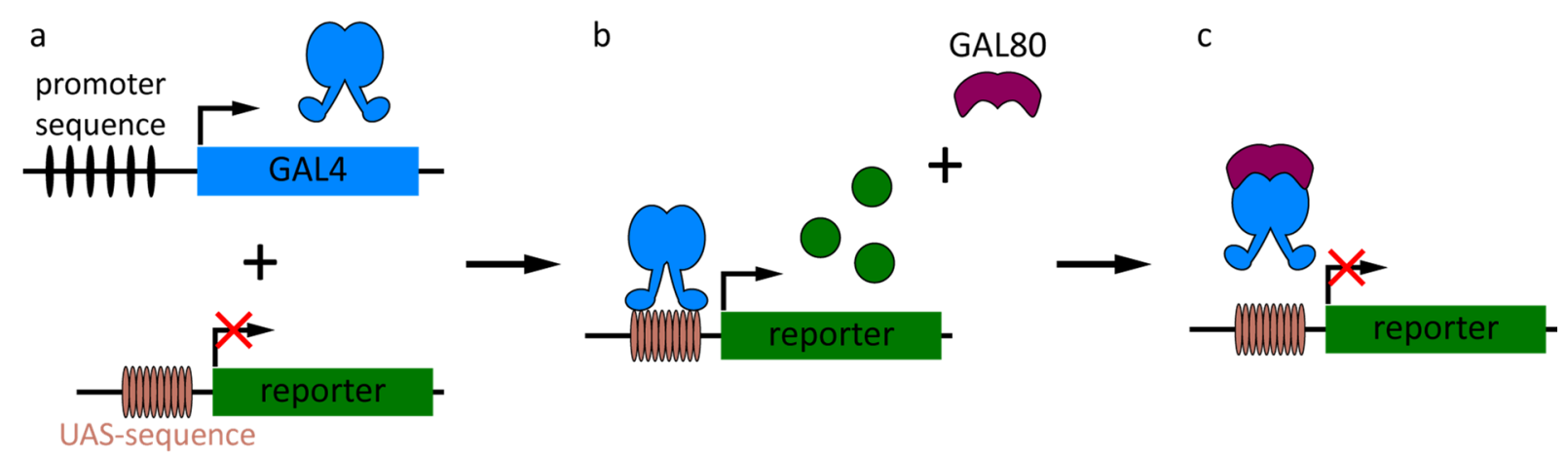

Figure 1.1: Gal4/UAS expression system that allows for cell type specific transgene expression. a The yeast transcription factor GAL4 is inserted downstream of an promoter sequence and expressed in cells in which this promoter is active. An e.g., effector or reporter protein sequence is inserted downstream of the upstream activating sequence (UAS) that does not lead to expression without the GAL4 transcription factor protein. The co-occurrence of both transgenes in one cell leads to the binding of GAL4 to the UAS sequence and consequently to expression of the e.g., reporter (b). If the GAL4 repressor protein GAL80 is expressed as well, it will bind to GAL4 thus leading to inactivation of GAL4 and repression of reporter protein expression (c).

\subsubsection{Mosaic Analysis with a Repressible Cell Marker}

Even though GAL4 driven expression provides a powerful tool to investigate specific types of neurons the localization of neuronal function to single neurons is of great interest. This was achieved with high precision by Lee and Luo (1999) who developed the mosaic analysis with a repressible cell marker (MARCM , Fig. 1.2). The MARCM technique is based on the FLP/FRT system (Golic and Lindquist, 1989) in which the yeast flippase (FLP) recombinase can recombine between two flippase recognition target (FRT) sites (Fig. 1.2 b). To avoid ubiquitous expression under GAL4 control the repressor protein GAL80 was introduced behind the FRT site. If GAL80 is expressed it 
binds to the GAL4 protein, thereby preventing the binding of GAL4 to the UAS-sequence (Fig. 1.2 a). The FLP (hs-FLP) is controlled under a heat shock promoter (hsp70; Ashburner and Bonner, 1979) allowing for desired FLP-expression only if a heat shock is applied (Fig. 1.2 b). To label only a subset of cells the heat shock has to be induced during mitosis of the mother cell. Furthermore, the mother cell has to carry homozygously FLP and FRT and only heterozygously GAL80. In this constellation the heat shock will induce FLP-activity that recombines at the FRT-sites, exchanging the GAL80 between chromosomes (Fig. 1.2 b). If this happens during mitosis, one daughter cell will be homozygous for GAL80, preventing GAL4 activity and subsequent UAS-dependent transgene expression (Fig. 1.2 c, bottom). The other daughter cell will be without GAL80, having full GAL4 activity that allows for the expression of the UAS-combined transgenes in a single neuron (Fig. $1.2 \mathrm{c}$, top). The timing of the heat shock further determines the likelihood of single cell labeling as the heat shock should induce recombination only in the last differentiation state of a cell lineage to remove the GAL80 transgene (Fig. $1.2 \mathrm{~d}$ ). If the daughter cell that has no GAL80 transgene anymore will further differentiate all upcoming cell clones will show transgene expression (Fig. 1.2 e and f).
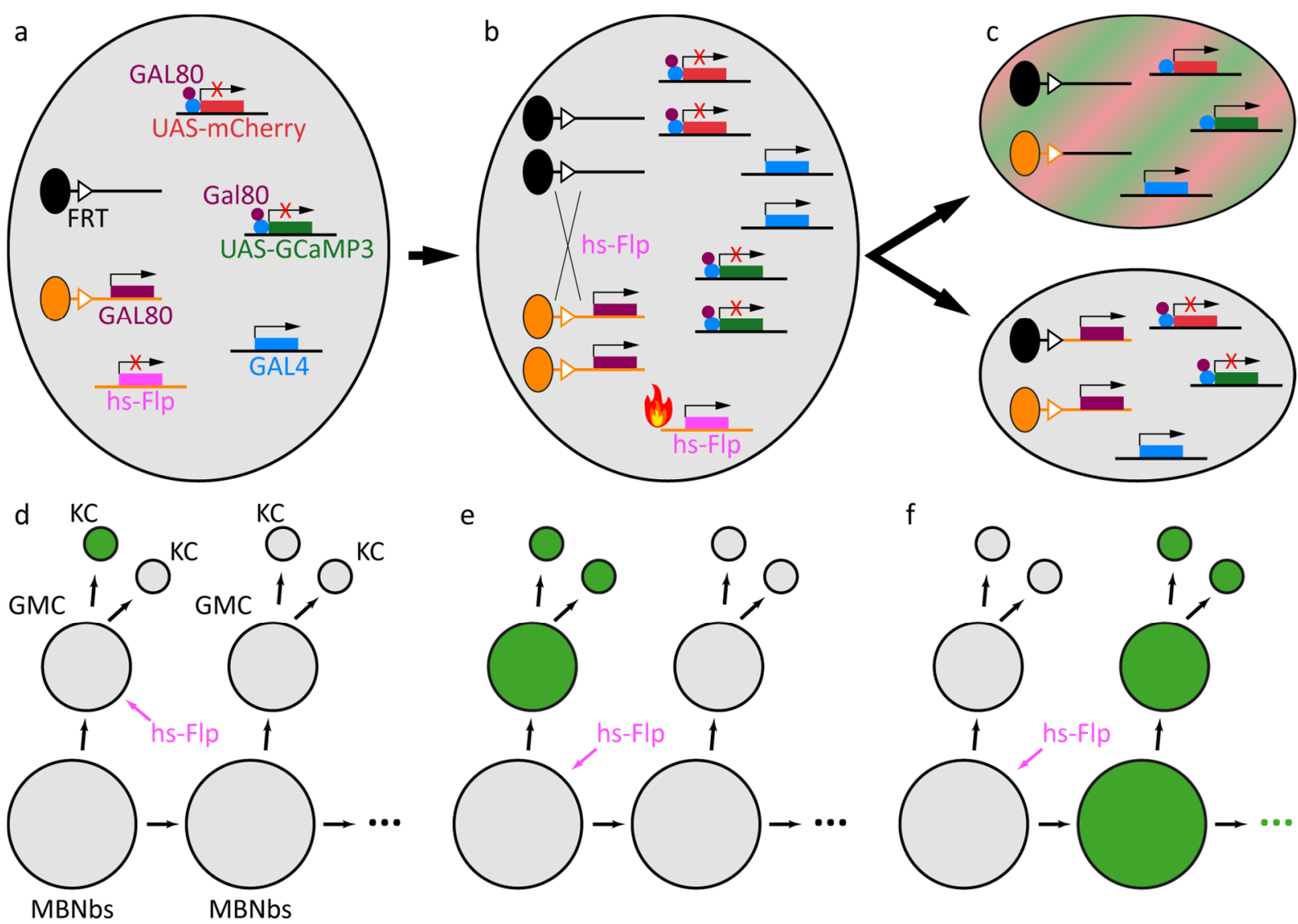

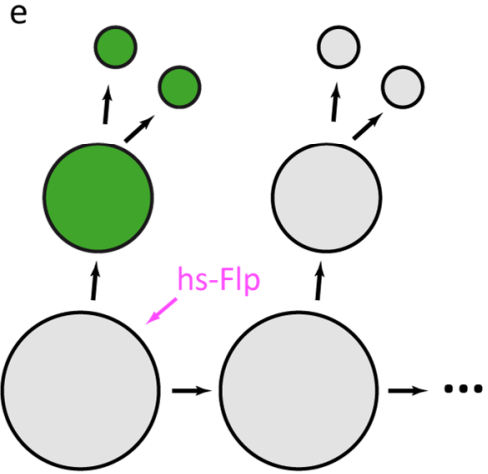

f

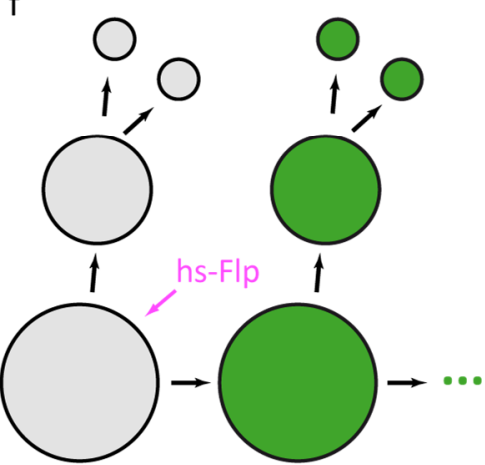

Figure 1.2: Mosaic analysis with a repressible cell marker (MARCM) in the developing mushroom body neuroblasts (M BNbs) modified after Lee and Luo (1999). MARCM can be utilized to drive expression of transgenes in single cell clones and is based on the GAL/UAS system. a Flippase recognition targets (FRT) are 
inserted in a homologous chromosome pair at which a heat shock inducible flippase (hs-FLP) can recombine. Furthermore, the cell expresses GAL4 and heterozygously the repressor GAL80 that inhibits GAL4 function. Thus, the UAS-constructs are not expressed. b During mitosis the chromosomes are duplicating and each daughter cell will get the same set of chromosomes leading to GAL80 mediated repression of UAS-transgene expression. To induce expression in one of the daughter cells the GAL80 has to be removed from this cell. This is done by inducing recombination via the hs-FLP that is under control of a heat shock promoter. This allows for temporal control of hs-FLP activity. If a heat shock is presented to the cell, the hs-FLP will recombine at the FRT sites exchanging chromosome arms. c The hs-FLP recombination leads to one daughter cell homozygous for GAL80 and therefore without UAS-transgene expression (lower panel) and the other daughter cell without GAL80 showing full transgene expression (upper panel). $\mathbf{d}$ This technique can be used to drive transgene expression in e.g., single KCs of the MB. The MB develops from four neuroblasts (MBNbs) each of which divides into a new $M B N b$ and a ganglion mother cell (GMC). The GMC finally differentiates into two KCs. With the new MBNb the differentiation cycle starts again. If the heat shock is applied during GM C division one of the KCs will express the transgenes of desire resulting in a single labeled cell. e If the heat shock is applied during MBNb division either the GMC will express the transgenes of desire and therefore the two differentiated KCs or the new MBNb and therefore the whole following cell lineage (f).

\subsubsection{Identification and Functional Characterization of Neurons and Circuits in Drosophila}

The above mentioned techniques are important milestones in the investigation of neuronal function in Drosophila. They can be utilized to express any protein of desire to manipulate or monitor neuronal functions and development. One protein has become indispensable in science: the green fluorescent protein (GFP) that was first purified from the jellyfish Aequorea victoria by Osamu Shimomura (Shimomura et al., 1962) and further improved and utilized by Martin Chalfie (Chalfie et al., 1994) and Roger Tsien (Heim et al., 1995; Tsien, 1998) for which these three scientist were awarded with "The Nobel Prize in Chemistry" 2008. The utilization of GFP was a breakthrough and changed fluorescence microscopy fundamentally as it was now possible to genetically label any cell of desire and investigate their anatomies. Later, many other fluorescent proteins were isolated emitting in different colors e.g., red fluorescing proteins like RFP and mCherry that were derived from DsRed (also known as drFP583) that was isolated from Discosoma sea anemones (M atz et al., 1999; Shaner et al., 2004; Zhang et al., 2002).

The mere expression of GFP in a neuron or a set of neurons delivers amazing anatomical insights into the neuronal architecture. But who connects to whom forming synaptic contacts in neuronal circuits? This question could be answered by a technique called GFP reconstitution across synaptic partners (GRASP) and was first developed in the nematode Caenorhabditis elegans (Feinberg et al., 2008) and later adopted for Drosophila (Gordon and Scott, 2009). In this technique the GFP protein is split into two non-fluorescent membrane-bound parts (CD4-spGFP1-10 and CD4spGFP11) that can be expressed in different neurons. Only if the two membranes and therefore the two parts come into close proximity the GFP protein will be fully reconstituted and can emit fluorescent light. This technique can reveal connectivity between neurons, but has the downside 
of being potentially non-specific to synaptic contacts. This problem was overcome by tagging one splitGFP part to the vesicle membrane specific protein synaptobrevin (syb:SpGFP1-10) enabling reconstitution only at synaptic sites (M acpherson et al., 2015). Furthermore, the strength of the GFP signal corresponds to the amount of activity of that neuron adding a functional aspect to this technique. In the course of this study additional GRASP variants of different colors were generated (cyan and yellow; (Macpherson et al., 2015).

In order to not only trace a neuron's anatomy but visualize its activity, the investigation of calcium currents are essential as action potentials open voltage gated calcium channels, increasing the intracellular calcium concentration that is needed for the transmitter release at the synapse (Katz and M iledi, 1965; Littleton et al., 1994). Therefore, calcium currents indicate neuronal activity that could be visualized with the development of the calcium-dependent GFP variant GCaMP (Nakai et al., 2001), which has been further developed and adapted thereafter. Here, the calcium-sensitive calmodulin was connected to a circularly permutated enhanced GFP via the M13 fragment of myosin light chain kinase that changes its conformation upon calcium influx leading to a higher fluorescence emission (Nakai et al., 2001). Other neuronal activity monitoring proteins rely on energy transfer from one fluorophore to another due to calcium binding (FRET e.g., cameleon or camgaroo, Fiala et al., 2002; Miyawaki et al., 1999; Yu et al., 2003) or indicate the synaptic vesicle release via detection of pH changes (e.g., synapto-pHluorin, M iesenböck et al., 1998).

Another important step in investigating neuronal function is the artificial activation or silencing of neurons as it allows for manipulation of neuronal activity or synaptic transmission determining the necessity of neurons in e.g., circuit functions. To test whether the function of a neuron is sufficient for a certain e.g., behavioral trait the artificial activation should lead to this behavioral output. If silencing the neuron leads to a disruption of the certain behavior it is furthermore required. These are key techniques to determine if neurons are part of a memory trace as their activation should lead to memory formation and their output block should disrupt memory formation (Gerber et al., 2004). To activate a neuron, light or temperature sensitive ion channels like the heat-activated outward rectifying cation Transient Receptor Potential (dTRPA1) channel (Rosenzweig, 2005; Viswanath et al., 2003), the light-sensitive cation channel Channelrhodospin2 (ChR2; Zhang et al., 2007b), or the red-light sensitive Channelrhodopsin variant CsCrimson (Klapoetke et al., 2014) were utilized. In order to silence a neuron the excitability can be reduced (e.g., Kir2.1, genetically modified $\mathrm{K}^{+}$-channel; Baines et al., 2001; White et al., 2001), the synaptic transmission reversibly altered (e.g., Shibire ${ }^{\text {ts }}\left(\mathrm{Shi}^{\mathrm{ts}}\right)$; thermosensitive, dominant-negative mutant form of dynamin; Kitamoto, 2001) or abolished (e.g., TeTxLC; Sweeney et al., 1995).

These tools can be expressed in neurons of desire by cloning them e.g., behind the UAS-sequence allowing for the investigation of neuronal substrates that are part of the memory trace. 
Introduction

Drosophila olfactory associative conditioning is one of the most intensively studied forms of associative learning as it can be efficiently utilized in the laboratory to investigate the mechanisms of associative learning (Busto et al., 2010; Tully and Quinn, 1985). In order to understand the mechanisms and performance of Drosophila in olfactory associative learning tasks the olfactory system has to be explained in detail.

\subsection{The Olfactory System of Drosophila}

Drosophila as a model organism has great advantages for the study of associative learning and memory. Besides the above mentioned genetic tools to manipulate and monitor neurons their nervous system is simpler compared to vertebrates comprising $\approx 100,000$ neurons of which many are identifiable and addressable. Odors play an essential role in the behavior of Drosophila as they are important to detect e.g., foraging sites, potential mates, and predators or hazards. Furthermore, it was shown that Drosophila is capable of learning contexts in olfactory association tasks (reviewed by Busto et al., 2010).

On the head of Drosophila two paired appendages are used to detect odor cues - the antennae and maxillary palps (Fig. 1.3 a, yellow circles). The antennae play the major role in odor detection and consist of 4 segments. Especially the third antennal segment (funiculus) as it houses approximately 420 sensory hairs, called sensilla (Fig. 1.3 b). The sensilla are divided into three types - basiconic, coeloconic, and trichoid housing, dependent on the type, two to four olfactory sensory neurons (OSNs, in total $\approx 1200$ ) expressing the odorant receptors (ORs) involved in odor detection (de Bruyne et al., 2001; Shanbhag et al., 1999; Stocker, 1994). The maxillary palps house 60 exclusively basiconic sensilla housing always pairs of OSNs (in total $\approx 120$, de Bruyne et al., 1999; Nayak and Singh, 1985). Odor molecules enter the sensilla through pores, where they reach the dendrites of OSNs and bind to the receptors. The co-expression of the odorant receptor coreceptor (ORCO or OR83b) is essential for OSN activation through odor binding (Larsson et al., 2004; Vosshall et al., 2000) but its exact role is still under debate (Nakagawa and Vosshall, 2009). OSNs that express the same or similar types of ORs (62 different receptor proteins; Robertson et al., 2003) project their axons onto the same one or two spherical structures (called glomeruli, GL) in the first olfactory brain center, the antennal lobe (AL, Fig. 1.3 b, Couto et al., 2005; Shanbhag et al., 1999; Vosshall et al., 2000). An AL consists of $\approx 54$ glomeruli (Grabe et al., 2015) that show combinatorial odor-dependent spatiotemporal activity patterns that are distinct for dissimilar odors and highly overlapping for similar odors (Barth et al., 2014; Gao et al., 2000; Hallem and Carlson, 2004; Wang et al., 2003). OSNs in the AL synapse onto $\approx 200$ projection neurons (PNs) 
that convey the odor information through three tracts (iACT, mACT, oACT) to the next level brain centers the mushroom bodies (MB) and the lateral horns (LH; Jefferis et al., 2001; Marin et al., 2002; Stocker et al., 1990; Wong et al., 2002). The dendrites of PNs are mainly uniglomerular (Stocker et al., 1990) providing a stereotypic response pattern for identifiable PNs across individuals. The $A L$ is furthermore innervated by $\approx 100$ local interneurons ( $L N$, excitatory or inhibitory) that mostly globally innervate all glomeruli and are involved in gain control, sharpening odor contrast, and modulation of odor sensitivity and discrimination ( $\mathrm{Ng}$ et al., 2002; Olsen and Wilson, 2008; Olsen et al., 2010; Parnas et al., 2013; Root et al., 2008; Stocker et al., 1990; Wilson and Laurent, 2005).

All PNs terminate in the LH (Fig. 1.3 b), which is involved in innate olfaction-guided behaviors (de Belle and Heisenberg, 1994; Gupta and Stopfer, 2012; Heimbeck et al., 2001) and has furthermore been shown to be stereotypically subdivided based on chemical properties, valence, and stimulus concentration (Fişek and Wilson, 2014; Grabe and Sachse, 2018; Jefferis et al., 2007). The majority of the PNs ( $\approx 150$, projecting in the $\mathrm{IACT}$ ) pass the MBs (Fig. $1.3 \mathrm{~b}$ ), forming pre-synaptic terminals (called boutons) in the dendritic region of the MB, the calyx (Aso et al., 2009; Jefferis et al., 2007; Marin et al., 2002; Stocker et al., 1990; Wong et al., 2002). The M Bs were shown to be the key structures in olfactory associative learning (reviewed by: Davis, 2005; Heisenberg, 2003; Keene and Waddell, 2007; M cGuire et al., 2005; Waddell, 2013) and will therefore be described in detail in the next section.
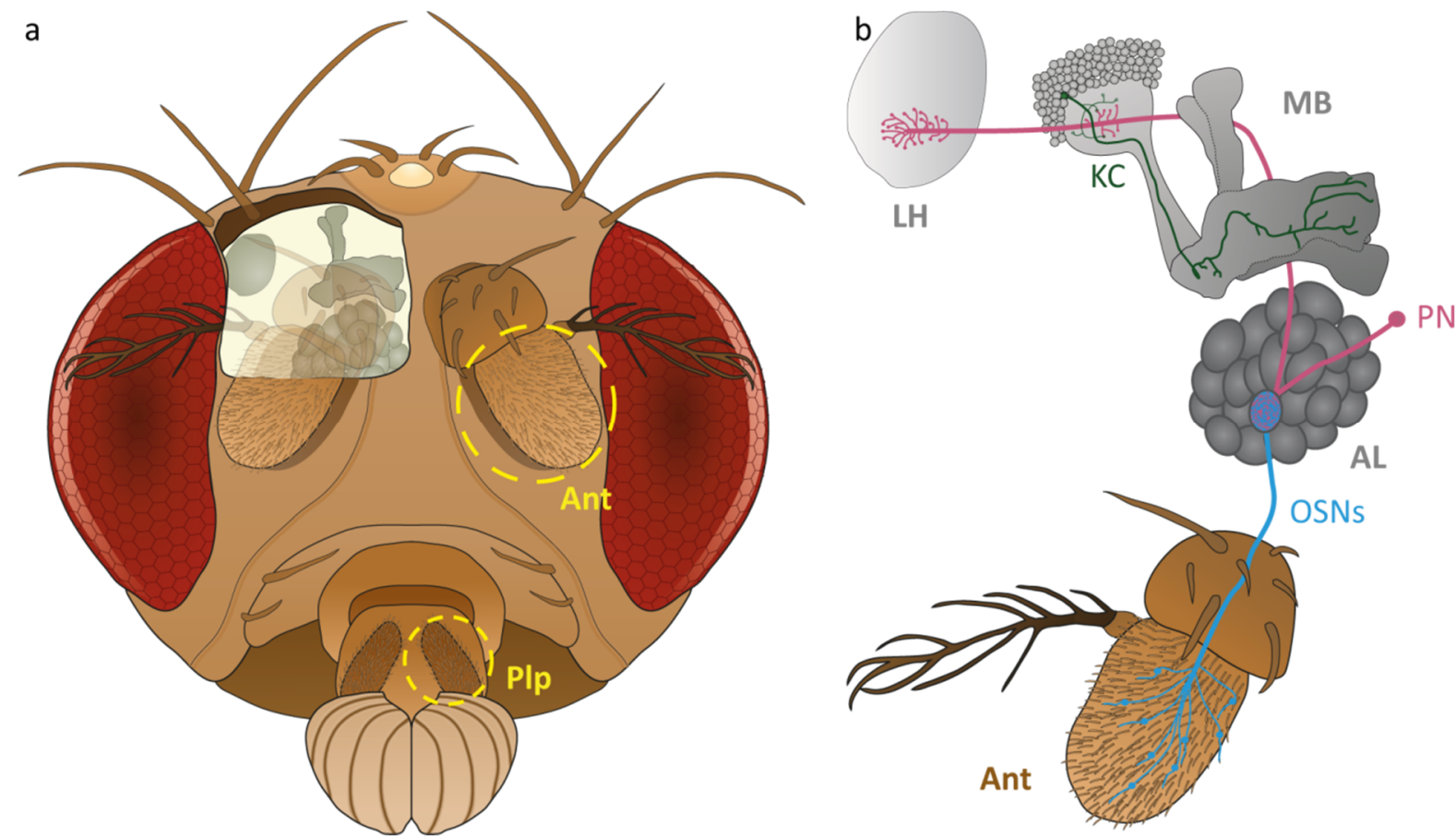

Figure 1.3: Schematic illustration of the Drosophila olfactory system. a Frontal view of the Drosophila head showing the two paired appendages (yellow dotted circles), the antennae (Ant) and palps (PIp). The upper left depicts a head opening exposing the brain and the main brain centers involved in olfaction (b). $\mathbf{b}$ 
Exemplified olfactory pathway of Drosophila. The dendrites of olfactory sensory neurons (OSNs) that take up odor information are housed in sensilla distributed over the Ant. OSNs that express the same OR converge in the first brain center of the olfactory pathway, the antennal lobe (AL) forming glomeruli with the dendrites of second order projection neurons (PN). PNs convey the information to the higher order brain centers. Most PNs pass the mushroom bodies (MB) forming bouton-like axon terminals conveying odor information to the MB intrinsic Kenyon cells (KC). The PN axons project further and terminate in the lateral horn (LH). A smaller set of PNs bypass the MBs projecting directly to the LH (not shown).

\subsection{The Mushroom Bodies - Key Structures in Associative Learning and Memory}

\subsubsection{The Mushroom Body Anatomy}

The mushroom bodies were first described as early as 1850 by Félix Dujardin (Dujardin, 1850). Kenyon first described the MB intrinsic cells in detail after whom they were called Kenyon cells (Kenyon, 1896a, 1896b). The MBs are common among insects and other arthropods (except crustaceans) and some annelids; however, they show diverse shapes and numbers of intrinsic KCs (Strausfeld, 1998; Strausfeld et al., 1998, 2009).

In the insect phylum, Drosophila is of course not an exception. The M Bs of Drosophila consist of $\approx$ 2000-2500 KCs (Fig. 1.4) whereby the numbers differ because of different counting techniques but can also change dependent on the flies' environmental conditions (Aso et al., 2009; Technau, 1984). The KC somata are located on the posterior dorsal surface of the brain surrounding their dendritic field (calyx) in a cap like shape (comparable to a mushroom cap, Fig. 1.4 a and c). They further project in a dense bundle (peduncle, no branching) straight to the anterior brain surface (Fig. $1.4 \mathrm{a}$ and $\mathrm{c}$ ) where they bend at a $90^{\circ}$ angle to the midline, forming the axonal lobe regions (dorsal to the AL, Fig. 1.4 b) where they heavily branch and partially bifurcate (Fig. 1.4). The development of the GAL4/UAS system shed more light onto the KC anatomy as enhancer trap lines showed a genetically predetermined subdivision of KCs (Yang et al., 1995). This classification was further confirmed and extended by antibody stainings against proteins that showed KC type specific expression levels subdividing the MBs into three main KC classes: $\gamma, \alpha^{\prime} / \beta^{\prime}$, and $\alpha / \beta$ (Crittenden et al., 1998). The $\gamma$-KCs form a horizontal lobe with their axonal branches. The $\alpha / \beta-K C S$ bifurcate right behind the peduncle forming the vertical $\alpha$-lobe and the horizontal $\beta$-lobe that is posterior-ventral to the $\gamma^{-}$-lobe. The $\alpha^{\prime} / \beta^{\prime}-K C$ s bifurcate as well, where the vertical $\alpha^{\prime}$-lobe partially winds around the $\alpha$-lobe and the horizontal $\beta^{\prime}$-lobe lays on top of the $\beta$-lobe and posterior to the Y-lobe (Fig. 1.4).

Further antibody stainings and Golgi impregnations as well as GAL4/UAS enhancer trap and promoter studies revealed that the three main KC classes could be further subdivided (Aso et al., 
2009, 2014a; Butcher et al., 2012; Lin et al., 2007; Strausfeld et al., 2003; Tanaka et al., 2008). In total they can be dived into 7 subclasses: $\gamma$-main $\left(\gamma_{m}\right), \gamma$-dorsal $\left(\gamma_{d}\right), \alpha / \beta$-surface $\left(\alpha / \beta_{s}\right), \alpha / \beta$-core $\left(\alpha / \beta_{c}\right), \quad \alpha / \beta$-posterior $\left(\alpha / \beta_{p}\right), \quad \alpha^{\prime} / \beta^{\prime}$-anterior-posterior $\left(\alpha^{\prime} / \beta_{\text {ap }}^{\prime}\right)$, and $\alpha^{\prime} / \beta^{\prime}$-middle $\left(\alpha^{\prime} / \beta_{m}^{\prime}\right)$. Furthermore, $\gamma_{d}$ and $\alpha / \beta_{p}-K C s$ do not project their dendrites in the main calyx but form an exclusive ventral and dorsal accessory calyx, respectively. Alternatively, the $\mathrm{\gamma}$-KCs can be subdivided based on their activity of CAMP response element binding proteins (CREB), showing a population of $\approx 350$ CREB positive $\gamma$-KCs $(\gamma$ CRE- $p$ ) and $\approx 170$ CREB negative $\gamma$-KCs $(\gamma$ CRE- $n$ ) (Yamazaki et al., 2018).
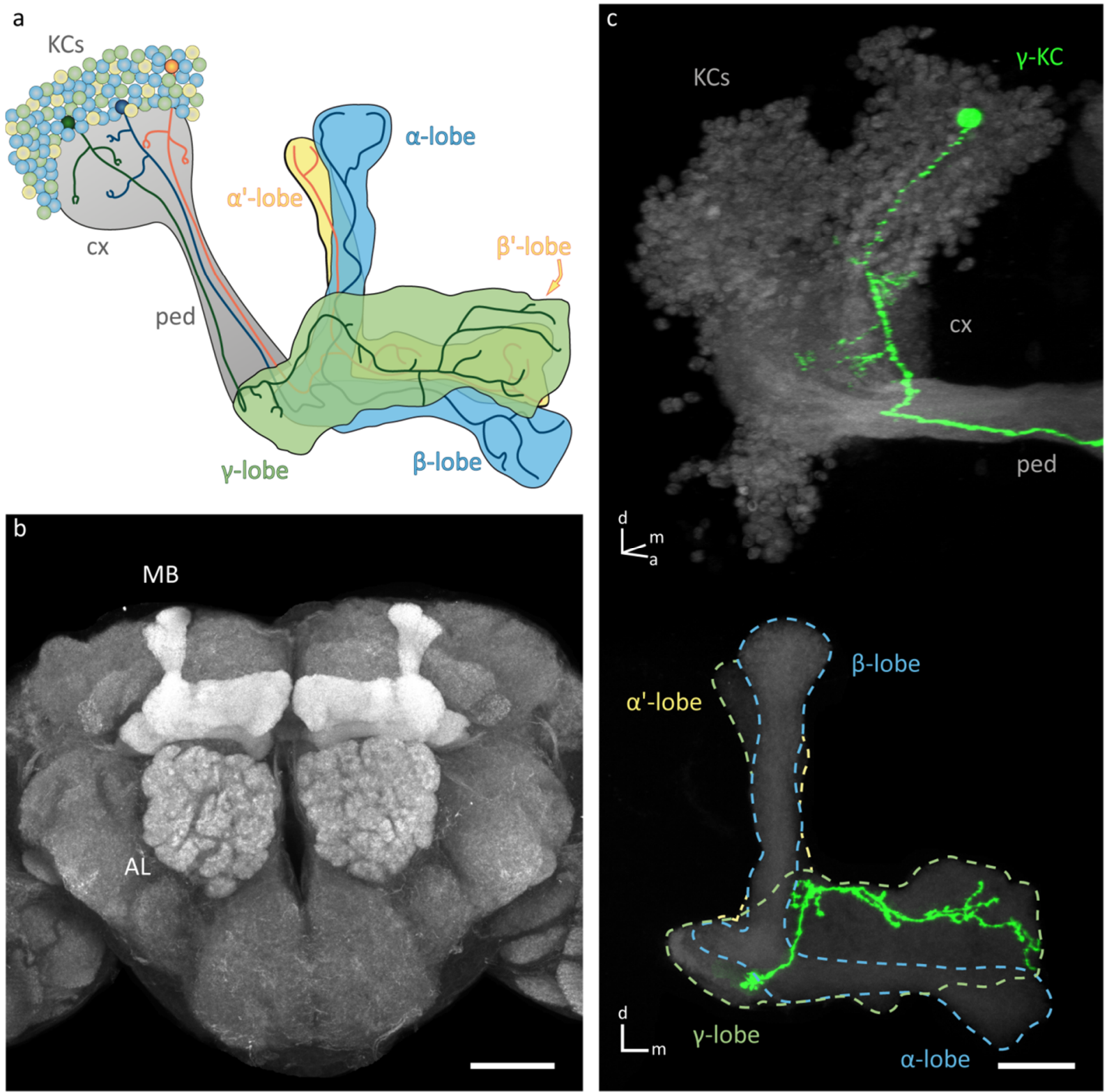

Figure 1.4: $M B$ architecture. a Schematic illustration of the MB architecture. The MB consists of $\approx 2000$ $2500 \mathrm{KCs}$ of which the cell somata of the three KC types $\left(\gamma-\mathrm{KCS}\right.$ - green, $\alpha / \beta-K C s-b l u e$ and $\alpha^{\prime} / \beta^{\prime}-\mathrm{KCS}-$ yellow) randomly distribute in the posterior dorsal surface of the brain. The KCs project their dendrites into the calyx (cx) where they terminate in $\approx 7$ claw-like structures. They further project their axons densely through the peduncle (ped) and form the characteristic lobes at the anterior dorsal brain surface. $\mathrm{Y}$-KCS 
form a horizontal lobe (green), $\alpha / \beta-K C s$ (blue) and $\alpha^{\prime} / \beta^{\prime}-K C s$ (yellow) both bifurcate forming a vertical and horizontal lobe each. Yellow arrow indicates the positioning of the $\beta^{\prime}$-lobe behind the $\gamma$-lobe. $\mathbf{b}$ Maximum projection of a confocal z-stack showing the brain neuropils. Most prominent are the MBs (strong white) and ventral to the MBs the ALs. Scale bar $=50 \mu \mathrm{m}$. c Confocal projections of MB intrinsic GCaMP3 expression (grey) and a single labeled $\mathrm{y}-\mathrm{KC}$ (green). The upper panel shows a 3D-projection of the posterior $\mathrm{KC}$ somata layer turned in an angle to depict the ped. The $\mathrm{\gamma}-\mathrm{KC}$ 's dendritic arbors branch in the $\mathrm{Cx}$ and further project through the ped. The lower panel displays the MB at the anterior level of the lobes showing the axonal branching of the $\gamma-\mathrm{KC}$ (green) inside the $\gamma$-lobe (green dashed outline). Furthermore, the $\alpha / \beta$ lobe (blue dashed outline) and the $\alpha^{\prime}-$ lobe are distinguishable. $a$ - anterior, $d$ - dorsal, $m$ - medial, scale bar $=20 \mu \mathrm{m}$. The 3D-projection in c was created with allowance from confocal data of Dr. David Vasmer.

\subsubsection{Development of the Mushroom Bodies}

The MB KCs derive from four different neuroblasts (MBNbs) in each hemisphere that proliferate from embryonic to late pupal stage and show no further proliferation during adulthood (Fig. 1.5; Ito and Hotta, 1992; Truman and Bate, 1988). The four MBNbs equally proliferate into all KC types but in a sequential manner with sharp transitions between KC types (Lee et al., 1999). They first form $\gamma$-KCs in the first 2.5 days after larval hatching (ALH), which interestingly bifurcate in larvae. Between 2.5 and 3 days ALH the transition between $\gamma^{-K C s}$ and $\alpha^{\prime} / \beta^{\prime}-K C s$ happens. $\alpha^{\prime} / \beta^{\prime}-K C s$ are formed until 4.5 days ALH. Between 4.5 and 5 days ALH the transition to forming $\alpha / \beta-K C s$ happens. This is also the time point at which pupation starts. These findings demonstrated that two KC types are formed during larval and one during pupal development (Lee et al., 1999). But not only the axonal lobes show a layered structure, the peduncle is subdivided as well, as newly born $\mathrm{KCs}$ project their neurites into the core thereby moving older $\mathrm{KC}$ neurites outwards forming concentric layers for all KC subtypes (Kurusu et al., 2002).

Holometabolous insects like Drosophila undergo drastic morphological metamorphosis during their pupal stage. In the MBs of Drosophila especially $\mathrm{Y}$-KCs undergo a drastic change (Lee et al., 1999): the whole calyx almost completely degenerates in the first 9 hours after pupal formation (APF) to the greatest extent in $\mathrm{\gamma}$-KCs. Furthermore, the bifurcating axonal branches of $\mathrm{\gamma}$-KCS completely degenerate until the end of the peduncle and are regrown until 24-36 hours APF, now forming only a horizontal lobe as seen in adult MBs. In contrast, the $\alpha^{\prime} / \beta^{\prime}-K C s$ appear somewhat immature before PF and maturate during the pupal stage. Nine days APF $(\approx 1$ day before eclosion), the MBNbs stop proliferation and the M B has its adult shape. 


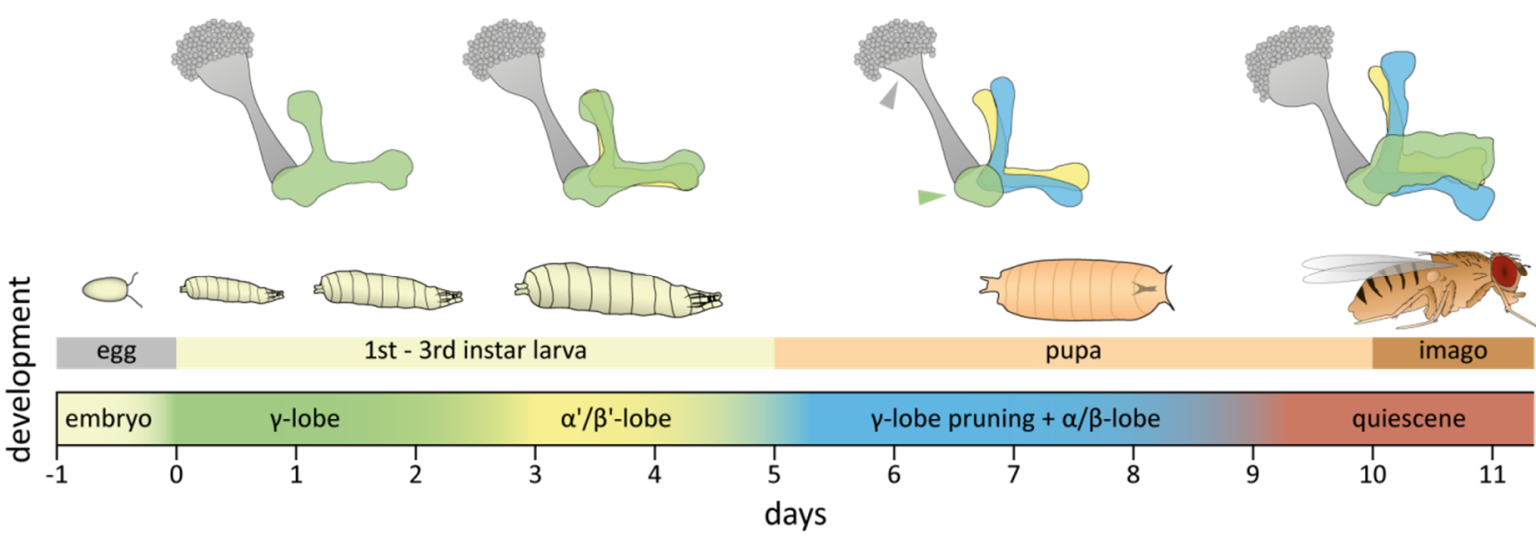

Figure 1.5: Drosophila and MB metamorphosis and development. Drosophila is a holometabolous insect that undergoes four drastic morphological changes during development (middle panel). In the first stage the embryo develops in the fertilized egg $\approx 21 \mathrm{~h}$ (day $-1-0)$. In the second stage the $1^{\text {st }}$ instar larva hatches out of the egg (day 0$)$ and grows over the next 5 days, molting two times $\left(2^{\text {nd }}\right.$ and $3^{\text {rd }}$ instar larva). With the first day of larval life the four MBNbs start to proliferate, forming first a bifurcating $\gamma$-lobe (green, upper panel). Approximately 2.5 days after larval hatching (ALH) the M BNbs switch to differentiate into $\alpha^{\prime} / \beta^{\prime}-K C S$ (yellow, upper panel). Approximately 4.5 days ALH the MBNbs start to differentiate into $\alpha / \beta$-KCs (blue, upper panel) and pupation is initiated where the larva enters the third stage (pupa) at day 5 . In the pupa the body morphology is completely rearranged. Also in the M Bs where shortly after the pupal formation the $\gamma$-KCs start to prune back to the end of the peduncle (green arrow head) and also the calyx shrinks due to dendrite retraction (grey arrow head). One day later the $\mathrm{Y}$-KCS start to regrow, now forming only a horizontal lobe. During pupa stage the MBs further develop until $\approx$ day 9 where the MBNbs stop proliferation. On day 10 the fly imago is fully developed and hatches, starting its adult life (fourth stage).

\subsubsection{Connectivities of the Mushroom Bodies}

The MBs get mainly olfactory input, which is conveyed by excitatory cholinergic PNs from the AL (Fig. 1.6 a; Turner et al., 2008; Yasuyama et al., 2002). PNs that project their axons through the iACT pass the MB calyx where 2-11 bouton terminals per PN connect to the $\mathrm{KC}$ dendrites (Wong et al., 2002). The number of PN boutons is stereotypic in similar PN classes but variable between different PN classes (Wong et al., 2002). There are three types of bouton shapes described, of which several can occur within a single PN axon (Butcher et al., 2012). The dendritic terminals of KCs form claw-like structures (5-7 per $\mathrm{KC}$ ) of which several claws ( $\approx 11$ per bouton) originating from different KCs grasp a single PN bouton (Butcher et al., 2012; Leiss et al., 2009; Yasuyama et al., 2002). The shape and number of claws is dependent on KC type (Butcher et al., 2012). Additionally, the GABAergic anterior paired lateral (APL) neuron projects into the calyx, synapsing onto KC claws and PN boutons, altogether forming the so-called micro glomeruli (Liu and Davis, 2009; Yasuyama et al., 2002). On one hand the connectivity of PNs to KCs was thought to be random by investigating anatomical features (Caron et al., 2013). On the other hand there is evidence that the connection is not completely random as PNs with the same physiological features tend to project onto the same KC (Gruntman and Turner, 2013) and furthermore, certain PN types show regionalized projection patterns into the calyx (Tanaka et al., 2004). Similarly, 
Introduction

dendritic arbors of KCs also show zonal project patterns based on KC type and birth order (Lin et al., 2007).

The GABAergic APL neuron is special in the sense that it is a single neuron per hemisphere. Its dendrites are inside the MB lobes and its axons project to the calyx and the peduncle, forming an MB intrinsic loop of information flow (Liu and Davis, 2009; Tanaka et al., 2008). Besides the cholinergic PN and GABAergic APL input to the calyx, OA immunoreactive processes were also found in the calyx (Strausfeld et al., 2003).

A second single neuron (per hemisphere), the dorsal paired medial neuron (DPM), projects exclusively inside the MB lobes (Ito et al., 1998; Tanaka et al., 2008; Waddell et al., 2000). Its transmitters are a neuropeptide processed from the amnesiac (amn) gene (Waddell et al., 2000), serotonin (Lee et al., 2011), and GABA (Haynes et al., 2015).

Immunoreactivity, as well as GRASP, experiments have shown that the MBs are heavily innervated by aminergic neurons e.g., dopamine (DA), serotonin (5HT), and octopamine (OA). Octopaminergic neurons only sparsely innervate the MBs; however, the KCs themselves show high and lobe-specific OA-receptor expressions (Pech et al., 2013; Sinakevitch and Strausfeld, 2006; Zhou et al., 2012) and are shown to be involved in appetitive memory (Huetteroth et al., 2015; Zhou et al., 2012). Besides the DPM neuron, other serotonergic neurons innervate the MBs as well, showing coarse segregated innervation patterns throughout the M Bs (Pech et al., 2013) and were shown to be involved in place memory and memory consolidation (Lee et al., 2011; Sitaraman et al., 2008).

Some of the most intensively studied aminergic neurons are the dopaminergic neurons (DAN) because of their immense importance in classical conditioning where they convey the punishing or rewarding stimuli. The innervation of DANs is special in the sense that two different DAN populations innervate specific regions in the MB lobes, tiling all lobes into five compartments (Fig. 1.6 b-e): $\gamma 1-\gamma 5, \alpha^{\prime} 1-\alpha^{\prime} 3+\beta^{\prime} 1-\beta^{\prime} 2$, and $\alpha 1-\alpha 3+\beta 1-\beta 2$ (Aso et al., 2014a; Claridge-Chang et al., 2009; Ito et al., 1998; Mao and Davis, 2009; Pech et al., 2013; Tanaka et al., 2008). One DAN cluster is the protocerebral anterior medial (PAM) cluster that innervates $\gamma^{3}, \gamma^{4}, \gamma^{\prime}, \beta^{\prime} 1, \beta^{\prime} 2, \alpha 1, \beta 1$, and $\beta 2$ (Aso et al., 2014a). The activity of PAM DANs was shown to be involved in appetitive memory formation (Burke et al., 2012; Kim et al., 2007; Liu et al., 2012). In contrast, the protocerebral posterior lateral (PPL) DAN cluster 1 innervates $\gamma^{1}, \gamma^{2}, \alpha^{\prime} 1, \alpha^{\prime} 2, \alpha^{\prime} 3, \alpha 2$, and $\alpha 3$ (Aso et al., 2014a) and was implicated in playing a major role in aversive memory formation (Aso et al., 2010, 2012; Claridge-Chang et al., 2009; Mao and Davis, 2009; Riemensperger et al., 2005). Additionally, a second PPL cluster (PPL2ab) innervates the calyx of the M Bs (M ao and Davis, 2009) and might be involved in trace conditioning rather than classical conditioning (Lüdke et al., 2018). Interestingly, recent studies, using electron microscope reconstructions, have shown that DANs are connected 
to $\mathrm{KCS}$ via reciprocal synapses, indicating a recurrent information flow between both synapses (Fig. 1.8 b; Eichler et al., 2017; Takemura et al., 2017).

A very important part of the MB circuitry is the output. Here, 34 MB output neurons (MBON), falling into 21 different cell types, were found having dendritic innervations in the MB lobes in a compartment-specific manner (Fig. 1.6 b-e; Aso et al., 2014a; Séjourné et al., 2011; Tanaka et al., 2008). The MBONs can be subdivided into three classes based on their main transmitters. There are eight glutamatergic MBONs innervating $\gamma^{4}, \gamma^{5}, \beta^{\prime} 2, \alpha 1, \beta 1$, and $\beta 2$; eleven GABAergic M BONs innervating the peduncle, $\gamma_{1}, \gamma^{2}, \gamma^{3}$, and $\beta^{\prime} 1$; thirteen cholinergic MBONs innervating $\gamma^{2}, \alpha^{\prime} 1, \alpha^{\prime} 2$, $\alpha^{\prime} 3, \alpha 2$, and $\alpha 3$; two of unknown transmitter where one is innervating $\gamma 1+\gamma 2$ and the other one $\gamma 4+\gamma 5$; an additional MBON of unknown transmitter expression is innervating the calyx. Of the 21 MBON types, 13 extend their arborizations to a single compartment and the remaining 8 types to two compartments. 13 M BON types innervate the whole volume of a compartment, whereas the remaining MBON types only innervate sub-regions of a compartment. M ost MBONs project their axons outside the MB except from 3 MBONs projecting to other MB lobe compartments, likely providing feedforward information flow. In addition, one DAN is also interconnecting MB lobe compartments (Aso et al., 2014a). Interestingly, the compartmentalization of MBONs highly overlaps with the tiled innervation pattern of DANs that is stereotypic across flies, suggesting a segregated functional role of these KC-DAN-MBON units (Aso et al., 2014a). Indeed, the compartmentalization has functional implications in the behavior of the fly. Depending on the M B lobe compartment innervated, the output of the respective MBONs drive approach or avoidance behavior. It is believed that the net output will determine the behavioral trait. This output can be modulated in the course of associative learning, shifting the output activity towards approach or avoidance (Aso et al., 2014b; Hige et al., 2015a, 2015b; Owald et al., 2015; Pai et al., 2013; Perisse et al., 2016; Plaçais et al., 2013; Séjourné et al., 2011).

In addition to the above mentioned neuronal innervations of the MBs, glia cells are wrapping around the MB lobes, the peduncle, and also intrude the calyx in a mesh-like shape (Aso et al., 2014a; Ito et al., 1998; Leiss et al., 2009). 

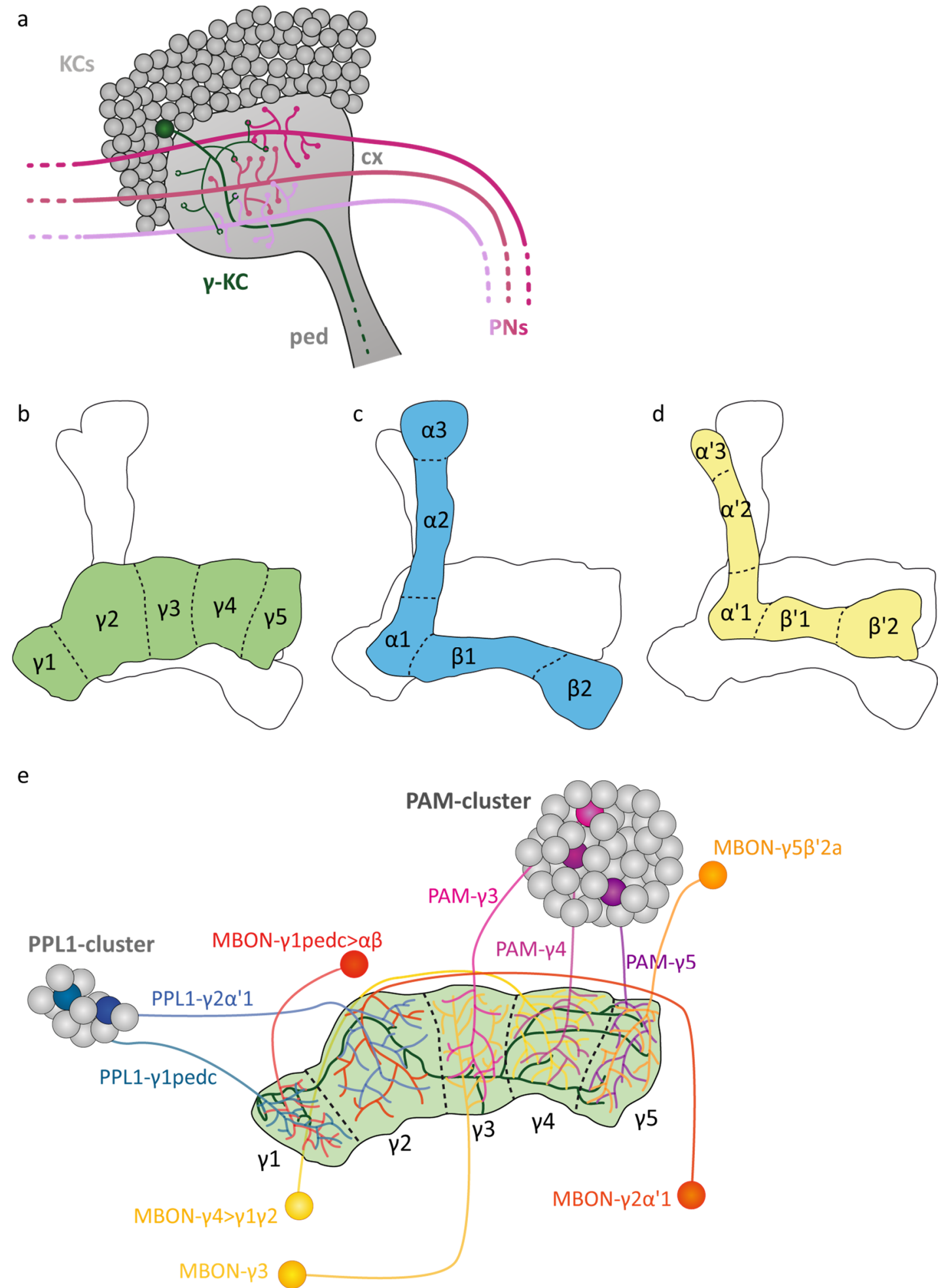

Figure 1.6: Exemplified connectivities and compartment-specific innervations of the $y$-lobe. a Schematic illustration of the olfactory input via PNs projecting their axons in the calyx of the MB. A KC has $\approx 7$ dendritic claws wrapping around PN axonal boutons. A KC receives rather random input from several different PNs. b-d Highly stereotypic extrinsic innervation of dopaminergic neurons (DAN) and MB output neurons (M BON) tiles the $\gamma^{-}(\mathbf{b}), \alpha / \beta$ - (c) and $\alpha^{\prime} / \beta^{\prime}$-lobes (d) in five distinct compartments each. e Exemplary extrinsic innervation of the $\gamma$-lobe showing the DAergic protocerebral posterior lateral cluster 1 (PPL1, blue) and the protocerebral anterior medial (PAM, purple) cluster as well as five different MBONs (orange). For simplicity only the axonal DAN and dendritic MBON innervations are illustrated (for further details see: Aso et al., 2014a). The axonal arborizations of PPL1 DANs are innervating $\gamma 1-2$, conveying aversive stimuli, whereas PAM DANs innervate $\mathrm{\gamma}^{3}-5$, conveying appetitive stimuli in a compartment specific, non-overlapping manner. Likewise, specific MBONs have compartment-specific dendritic innervation in the $y$-lobe, showing 
strong overlap with the respective DANs. Activation of MBONs innervating $11-3$ mediates approach behavior while activation of M BONs innervating $\gamma 4-5$ mediates avoidance behavior.

\subsubsection{Physiology of KCs}

The majority of KC input is olfactory, conveyed by cholinergic PNs (Turner et al., 2008; Yasuyama et al., 2002). Approximately $60 \%$ of the PNs respond stereotypically to an odor, coding the odor identity as a function of their spatiotemporal stereotypic OSN inputs (Wang et al., 2003; Wilson et al., 2004). PN axonal boutons semi-randomly connect to the dendritic claws of KCs (Caron et al., 2013; Gruntman and Turner, 2013; Lin et al., 2007; Tanaka et al., 2004). KC dendrites possess on average 5-7 claws (Butcher et al., 2012; Lee et al., 1999; Leiss et al., 2009) of which multiple must receive input to elicit spiking in KCs (Gruntman and Turner, 2013). This connectivity leads to a transformation from broadly tuned PN odor response to a sparse odor representation in the MBS where only $\approx 5 \%$ of the $\approx 2000 \mathrm{KCS}$ respond to one specific or several odors (Honegger et al., 2011; M urthy et al., 2008; Turner et al., 2008) similar to other insects e.g., the locusts (PerezOrive, 2002; Stopfer et al., 2003). In both Drosophila and locusts, not only the responsiveness is sparse in KCs but also spiking rates are low during odor stimulations and KCs show barely any spontaneous activity. The sparse odor code is further established and modulated through the GABAergic inhibitory feedback loop of the APL neuron (Lin et al., 2014a).

The advantage of such a sparse coding system lies in the high coding space - the fewer elements of a coding system are used for a codon, the more non-overlapping combinations are possible. Furthermore, sparse coding reduces synaptic interference that is important for memory formation. If a KC would respond to multiple odors and is trained to one, the presentation of the other odors could disturb the memory readout (Hige et al., 2015a; M asse et al., 2009; Olshausen and Field, 2004; Spanne and Jörntell, 2015). Sparse coding is also important for the learned discrimination of similar odors that is mediated by the APL neuron (Lin et al., 2014a). The block of APL-output via Shits expression showed a strong decrease in the discrimination performance (Lin et al., 2014a). Sparse odor coding can also be found in vertebrates in the piriform cortex that also receives strong olfactory input in a non-spatially distinct manner where only small nonstereotypic subsets of neurons are activated upon odor stimulation (Poo and Isaacson, 2009; Stettler and Axel, 2009). Additionally, the piriform cortex was implicated in aversive and appetitive associative learning that is independent of spatially distributed neuronal activation (Choi et al., 2011).

If a KC is finally activated by e.g., an odor, what is the neurotransmitter conveying the information to e.g., MBONs? It was shown just recently that the main neurotransmitter of KCs is acetylcholine 
Introduction

(ACh), regardless of KC type (Barnstedt et al., 2016). Furthermore, KCs were shown to co-express short neuropeptide $F$ (SNPF), a homolog of the mammalian NPY (Brown et al., 1999), but interestingly not in $\alpha^{\prime} / \beta^{\prime}$ - and $\alpha / \beta_{c}-K C s$ (Johard et al., 2008).

\subsection{Learning and Memory in Drosophila}

\subsubsection{Synaptic Plasticity - The Key to Memory Formation}

In a natural situation, the adaptation of behavior to certain environmental stimuli requires neural computations relying on sensory processing and experience. The integration of sensory inputs and experience-based memory components requires neuronal circuits that store and process these memory traces. Memory traces can potentially be activity, molecular or structural changes occurring in neurons. These changes have to be plastic in order to react to newly appearing changes. The groundwork for the investigation of synaptic plasticity was laid in the first half of the last century by Hebb and Konorski, and even before them by Cajal (Cajal, 1894; Hebb, 1949; Konorski, 1948). Their work proposed mechanisms in which small synaptic structures change in the course of learning. These changes occur on a short term and long term scale involving alterations of the efficiency of voltage gated calcium channels, the probability of vesicle release and pool size, number of release sites and the number and efficiency of post-synaptic receptors (see reviews: Dudai, 2004; Goyal and Chaudhury, 2013; Kandel, 2001; Kandel et al., 2014; Takeuchi et al., 2014; Wang et al., 1997). Synaptic plasticity is a prerequisite for the formation of memory traces (Martin et al., 2000; Takeuchi et al., 2014) and is mainly mediated by the CAMP/PKA pathway (Kahsai and Zars, 2011; Kandel, 2012). Furthermore, the stabilization of long term synaptic plasticity requires de novo protein synthesis (Bailey et al., 1996; Dudai, 2004; Tully et al., 1994). However, synaptic plasticity does not necessarily have to lead to only facilitation synaptic depression is also an essential mechanism of reducing synaptic efficiency that is important in the context of learning (Bear and Malenka, 1994). Synaptic plasticity provides the key mechanism to reorganize the neuronal network on a physiological and anatomic level, allowing for the appropriate behavioral output to a change in the environment. In Drosophila, synaptic plasticity is located mainly in the MB circuit and will be explained in detail in the following sections. 
Introduction

\subsubsection{The Memory Phases in Drosophila}

The field of learning and memory in Drosophila took off in the '70s of the past century, now almost 50 years ago, when it was shown that Drosophila can perform in operant conditioning paradigms (Quinn et al., 1974). In later experiments (Tully and Quinn, 1985) it was shown that Drosophila is capable of performing in association tasks in classical conditioning paradigms that Pavlov described already in his famous experiments with salivating dogs (Pavlov, 1906). They paired an odor (neutral stimulus - NS) with an electric shock (US) or paired light of a certain wavelength (NS) to the bitter tasting quinine (US) and found that flies learned to avoid the odor or wavelength that was paired with the aversive stimuli (CS+) in a two-choice situation (Quinn et al., 1974). Shortly after that, two memory components were found: one of which is resistant to cold shock anesthesia (ARM), whereas the other component is cold shock sensitive (ASM; Quinn and Dudai, 1976). Flies cannot only be trained to associate aversive stimuli to odors but also appetitive stimuli like sucrose (Tempel et al., 1983). However, appetitive learning is dependent on the feeding state. Fed flies do not perform well in appetitive conditioning, so flies need to be starved (Tempel et al., 1983). After all, four different memory phases could be described for Drosophila (Fig. 1.7): short term memory (STM ), that is protein synthesis independent, lasting up to an hour; middle term memory (MTM) lasting up to three hours; a cold shock sensitive long term memory (LTM) that is dependent on de novo protein synthesis, lasting up to 7 days; and a cold shock anesthesia resistant, protein synthesis independent long term memory (ARM) lasting up to 3 days (Tully and Quinn, 1985; Tully et al., 1994; see also review by: Heisenberg, 2003). STM is usually induced by single training trials, either aversive or appetitive. The induction of LTM formation differs in terms of which reinforcement is used. To induce aversive LTM , 5-10 training trials are needed that are spaced with 15 min resting periods (Tully et al., 1994). To induce appetitive LTM, only a single training trial is needed, but flies have to be starved (Krashes and Waddell, 2008; Tempel et al., 1983). 5-10 training trials without resting periods in between (massed training) induce protein synthesis independent ARM. Furthermore, single trial ARM is established in aversive training after $\approx 30 \mathrm{~min}$ and in appetitive training after $\approx 100 \mathrm{~min}$ (Tempel et al., 1983). Taken together, these findings suggest differential mechanisms for aversive and appetitive memory formation.

Clearly, it is remarkable that such a small organism with a comparably low number of neurons is capable of complex learning tasks but is also telling us again that size or numbers don't matter but what the system can accomplish. And of course, the question arose which structure in the brain is involved in learning and memory tasks?! 


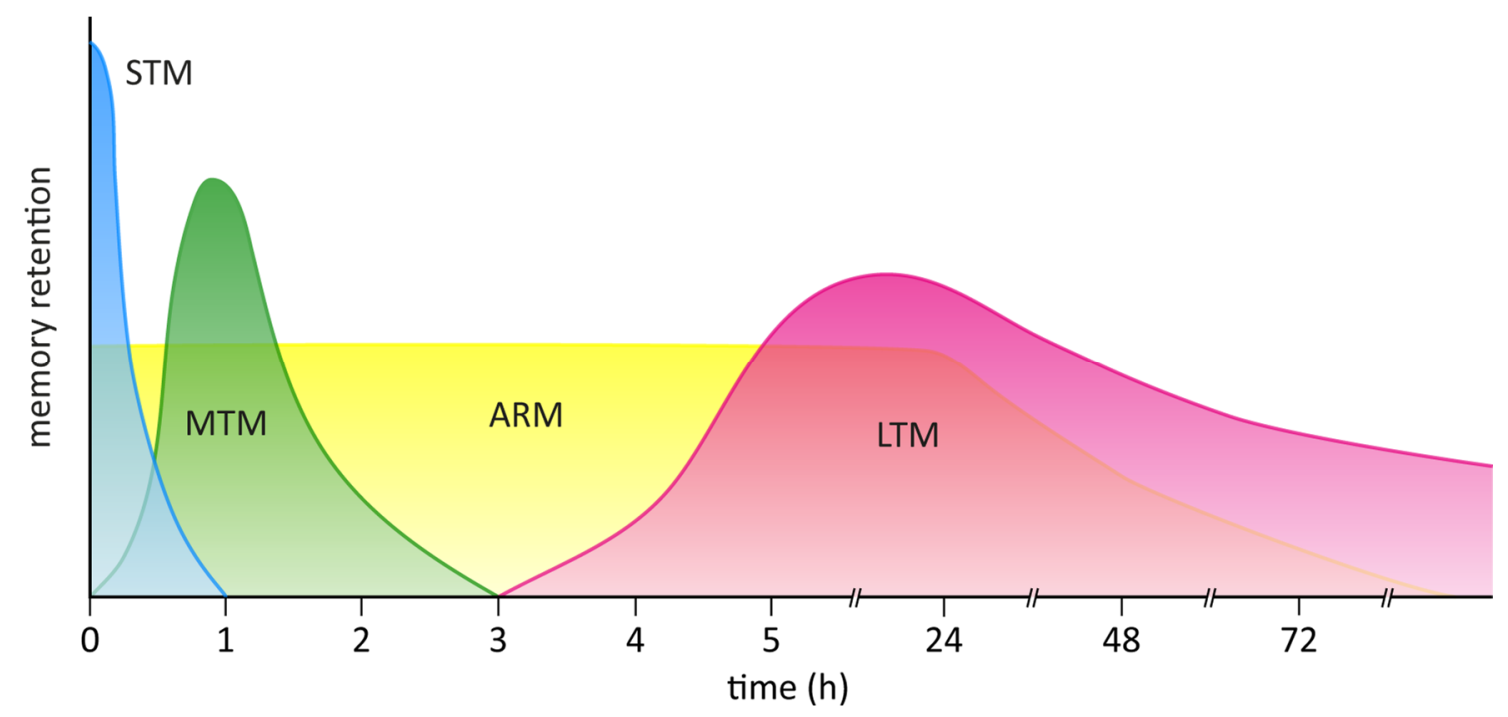

Figure 1.7: Memory phases of Drosophila modified after Heisenberg (2003) and Davis (2011). Drosophila shows different memory phases after olfactory associative conditioning: A short term memory (STM) component lasting up to an hour induced by single training trials; a middle term component (M TM) lasting up to three hours; and a long term, protein synthesis dependent component (LTM) that can last up to 7 days. In aversive associative conditioning LTM is formed after 10 training trials spaced by 15 min but needs only 1 training trial in appetitive conditioning. All of these memory phases are anesthesia sensitive and can be erased by e.g., a cold shock. However, Drosophila exhibits an additional protein synthesis independent memory component that is furthermore anesthesia resistant (ARM) and present throughout the first $24 \mathrm{~h}$ after training decaying over the next two days. It can be formed only after 10 massed training trials with no pauses in between.

\subsubsection{Learning M utants}

Quickly, the MBs became the focus of attention as the analysis of learning mutants and their protein products could be assigned to the MBs (Davis, 1993; Han et al., 1992; Nighorn et al., 1991; Skoulakis et al., 1993). By ethylmethanesulfonate treatment, causing mutagenesis, the first learning mutants that performed weakly after aversive olfactory conditioning were discovered in flies having a mutation in a gene named dunce (Dudai et al., 1976). It was found that dunce encodes for a CAMP phosphodiesterase and that its mutation causes elevated CAMP levels, implicating a role for CAM P in learning (Byers et al., 1981; Chen et al., 1986; Davis and Kiger, 1981)

Another classical learning mutant is rutabaga (rut), which was found to affect the responsiveness of adenylate cyclase (AC) to $\mathrm{Ca}^{2+} /$ calmodulin ( $\mathrm{Ca}^{2+} / \mathrm{CaM}$, a calcium binding protein motif), leading to loss of cyclase activity and in turn low levels of CAM P, causing poor performance in associative learning tasks (Levin et al., 1992; Livingstone et al., 1984).

A mutation in the DCO gene that encodes the catalytic subunit DCO, as well as in the RI catalytic subunit of the CAMP-dependent protein kinase (PKA), showed a reduction in PKA activity and 
reduction in learning performance especially in STM and M TM (Foster et al., 1988; Goodwin et al., 1997; Skoulakis et al., 1993).

The downstream target of PKA is the CAM P response element binding protein (CREB), which acts as a transcription factor for genes with CRE-binding sequences (see review by: Tully, 1996). The two isoforms (CREB-a and CREB-b) act antagonistically to each other, either activating or repressing CRE-dependent transcription, respectively. The activation of CREB-b suppressed expression of LTM, but interestingly the activation of CREB-a induced LTM already after a single training trial without changing LTM performance levels (Tully, 1996; Yin and Tully, 1996; Yin et al., 1995, 1994). The feeding of cyclohexamide (CXM ), a protein synthesis inhibitor, led to disruption of LTM formation (Tully et al., 1994), further demonstrating the necessity of protein synthesis to form LTM.

A mutated G-protein $\left(G \alpha_{s}\right)$ that was restrictively expressed in the MBs led to a constitutive activation of $A C$ in the KCS, which in turn abolished olfactory learning, indicating the role of $\mathrm{G}$ proteins and the MB itself in learning and memory (Connolly et al., 1996).

The above mentioned mutation analyses and the findings that products of dunce, rut, and DCO are enriched in the MBs (Nighorn et al., 1991; Skoulakis et al., 1993) highlight the importance and involvement of a cellular calcium-dependent CAMP pathway in learning and memory that is M B intrinsic.

Another important mutation that affects learning was found in flies in which the dopadecarboxylase (Ddc) gene was mutated (Livingstone and Tempel, 1983; Wright et al., 1981). These flies showed temperature-dependent decrease in DA and $5 \mathrm{HT}$ levels that proportionally affected the learning performance (Tempel et al., 1984). These findings indicated the importance of neurotransmitters in learning and memory.

Two additional learning mutants were discovered that first linked learning to the MBs: The mushroom body deranged and mushroom body miniature mutants were shown to have gross defects in the structure of the MBs and perform weakly after aversive and appetitive conditioning both in larvae and adults (Heisenberg et al., 1985).

Further evidence came from experiments in which larvae were fed hydroxyurea, killing MBNbs. This treatment led to flies having, in most cases, no M Bs (M B-less) which in turn did not learn in aversive conditioning experiments (de Belle and Heisenberg, 1994).

As mentioned above the elimination of DA production leads to learning deficits (Tempel et al., 1984). The G-protein coupled DA-receptor dDA1 was shown to be enriched in the MBs (Kim et al., 2003) and a mutation in this receptor, named dumb, showed the importance of DA signaling in learning as it impaired aversive and appetitive memory (Kim et al., 2007). 
Introduction

\subsubsection{The MB Circuit and its Role in Learning and Memory}

One of the most exiting questions in neuroscience is: how is learning and memory accomplished in the brain? And furthermore, how can two stimuli be associated with each other at the physiological level? As described above, the MBs were shown to be involved in learning and memory, so it appears likely that the KCs are one site at which learning potentially happens. However, the MB circuitry suggests the involvement of other neuron groups such as DANs and MBONs, as they heavily innervate the M Bs (Aso et al., 2014a).

\section{The role of DANs}

It was shown in classical conditioning experiments that flies can associate aversive or appetitive stimuli - the US - to an odor - the CS (Quinn et al., 1974; Tempel et al., 1983), whereby the KCS carry the odor information and DANs were shown to convey the information of the US. Interestingly, compartmentalized DAN innervation was found in the MB (Aso et al., 2014a), showing distinct functions. The DANs of the PPL1 cluster play a major role in aversive associative memories as they were shown to convey the electric shock stimulus (Cohn et al., 2015; Riemensperger et al., 2005) and are necessary for aversive conditioning (Aso et al., 2010; Riemensperger et al., 2005; Schwaerzel et al., 2003). Artificial activation of these DANs via the THGAL4 (tyrosine hydroxylase) driver line could substitute the natural US application in aversive conditioning (Aso et al., 2010, 2012; Claridge-Chang et al., 2009), indicating the importance of the PPL1 cluster in aversive memory formation. Furthermore, the PPL1 cluster was also shown to convey other noxious or unpleasant stimuli information to the M B e.g., heat (Galili et al., 2014) or bitter taste (Das et al., 2014). Interestingly, different DANs in the PPL1 cluster are involved in different memory retention times after aversive conditioning (Aso et al., 2012).

On the contrary, the DANs of the PAM cluster were shown to play a major role in appetitive memory as they convey the rewarding stimulus to the KCs (Burke et al., 2012; Cohn et al., 2015; Liu et al., 2012). In addition, OA is also necessary for appetitive memory (Schroll et al., 2006; Schwaerzel et al., 2003), but interestingly not via those OA neurons (OAN) directly projecting onto KCs (Burke et al., 2012). Instead, OANs connected to DANs expressing the OA receptor OAM B in PAM-DANs are required to signal the sweet taste of the sugar stimulus (Burke et al., 2012). Interestingly, there is a difference in memory formation dependent on sweet taste and nutritional value. Olfactory conditioning with non-nutritional sweet-tasting arabinose can form only STM and weak LTM whereas nutritional sweet-tasting sucrose or fructose induced robust STM and LTM indicating the role of nutritional value in enhancing sweet-taste conditioning (Burke and Waddell, 2011). Sweet taste memory is induced via the OAN to DAN connection (Burke et al., 2012), 
whereas nutritional memory is $\mathrm{OA}$ independent and signaled via different PAM-neurons projecting to $\gamma 5$ (Burke et al., 2012; Huetteroth et al., 2015).

The type of memory - either appetitive or aversive - depends on the type of US (e.g., sugar or electric shock, respectively) that is paired with the odor. As mentioned above (and see also 1.4.3), the US is conveyed by distinct DAN clusters that tile the MB lobes into 15 compartments. A comprehensive study has shown the involvement of these DANs by investigating subpopulations within those clusters (Aso and Rubin, 2016). Aso and Rubin (2016) showed that subpopulations of PPL1 DANs innervating $\gamma_{1}, \gamma^{2}, \alpha^{\prime} 1, \alpha^{\prime} 2, \alpha 2$, and $\alpha 3$ are sufficient to induce aversive memories and subpopulations of PAM DANs innervating $\gamma 5, \beta^{\prime} 2 a, \beta 1$, and $\beta 2$ are sufficient to induce appetitive memories. Interestingly, each subpopulation of either PPL1 or PAM is different in its formation and retrieval of STM or LTM as well as its stability against memory decay after repeated odor exposure, indicating very specific roles for the different MB compartments (for detailed description see: Aso and Rubin, 2016). Moreover, DANs do not only simply convey punishment or reward, their signaling of the US is also dependent on the internal state e.g., feeding status, thirst, or arousal which modulate DAN activity (Cohn et al., 2015; Huetteroth et al., 2015; Lin et al., 2014b).

\section{Coincidence detection}

Because KCs signal the odor and the DANs the US it is likely that the MB-DAN circuit acts as the coincidence detector responsible for modifying the output of KCs to their downstream targets the M BONs (Fig. 1.8 c). This assumption was emphasized by a study imaging CAM P levels in the MBS showing that the coincident activation of KCs and DA application leads to a synergistic rut-AC mediated increase in CAMP levels that is greater than the sum of the mere $\mathrm{KC}$ activation or DA application (Tomchik and Davis, 2009). The odor-induced activation of KCs leading to calcium influx in the axons (Akalal et al., 2010; Wang et al., 2008; Yu et al., 2006) presumably activating $\mathrm{Ca}^{2+} / \mathrm{CaM}$ and the coincident US-induced G-protein activation via the DA-receptor dDA1, leads to the synergistic activation of the rut-AC activating the downstream CAMP pathway, which is important for learning (Connolly et al., 1996; Dudai et al., 1976; Gervasi et al., 2010; Goodwin et al., 1997; Kim et al., 2007; Levin et al., 1992; Neve et al., 2004; Tomchik and Davis, 2009; Tully, 1996). Thus, the CAMP pathway is needed for coincidence detection and is therefore the basis for classical conditioning in general, independent of retention time (Blum et al., 2009). It is important to note that the timing of the US to the CS is essential for the type of memory that is formed (Tanimoto et al., 2004). When DANs that usually convey aversive stimulation (e.g., PPL1- $p 1$ pedc) are paired shortly after odor onset, an aversive memory is formed. However, if the same DANS are activated 20 - $60 \mathrm{~s}$ before odor onset an appetitive memory is formed indicating the 
Introduction

predictive nature of the odor, which seemingly signals the end of the aversive stimulation (Aso and Rubin, 2016; Tanimoto et al., 2004).

\section{The role of KC-types}

The fact that the MB consists of different KC types lets one assume that these types might play distinct roles in learning and memory. And indeed, a functional division was found for the different KC types. The CAMP pathway was shown to play a major role in STM as rut-rescues could restore STM especially in $\mathrm{Y}$-KCS (Akalal et al., 2006; Blum et al., 2009; Zars et al., 2000). The $\mathrm{\gamma}$-KCS were also found to be required for a rut-dependent aversive MTM, which is formed and retrieved from $y$-KCs (Xie et al., 2013). Important to note, the $\gamma_{d}$-KCs - which get only visual input from visual projection neurons in the ventral accessory calyx - are only involved in visual but not olfactory learning (Vogt et al., 2016). The functional division of $\psi$-KCs into $\gamma C R E-p$ and $\gamma C R E-n$ (see 1.4.1) showed opposing effects of these two types in learning and memory (Yamazaki et al., 2018):

Output blockage of $ү$ CRE-p by expression of Shit ${ }^{\text {ts }}$ showed that these subtypes are required for acquisition, consolidation and retrieval of aversive MTM and LTM. When $\gamma$ CRE- $n$ are blocked, appetitive M TM consolidation and retrieval is impaired. Artificial activation of $\gamma C R E-p$ with dTrpA1 showed a decrease in appetitive memory but an enhancement of aversive memory and vice versa if $\gamma$ CRE-n were artificially activated. Furthermore, the two subtypes were shown to inhibit each other upon their activation. Interestingly, the activation or the respective converse inhibition of a subtype could, to a lesser degree, substitute an aversive ( $\gamma$ CRE- $p$ ) or an appetitive ( $\gamma C R E-n)$ US. LTM formation was found to also be dependent on the CAMP pathway, specifically in $\alpha / \beta-K C S$, as the rut-rescue restored LTM, which was even increased in concert with rut-rescues in $y-K C S$, demonstrating the synergistic effect of $\gamma-K C s$ and $\alpha / \beta-K C S$, suggesting a transformation of memory phases from one KC type to another (Blum et al., 2009; Cervantes-Sandoval et al., 2013). Experiments with Shits -expression in MB subsets (blocking neuronal transmission) showed that the $\alpha / \beta-K C S$ are needed for memory retrieval of all retention times in aversive and appetitive conditioning (Cervantes-Sandoval et al., 2013; Dubnau et al., 2001; McGuire, 2001; Xie et al., 2013). Furthermore, if the output of both $\gamma-\mathrm{KCS}$ and $\alpha / \beta$-KCs is blocked simultaneously, memory retrieval of STM and MTM is completely abolished (Xie et al., 2013). The release of SNPF by $\mathrm{Y}^{-K C S}$ and $\alpha / \beta-K C S$ is required for appetitive memory presumably acting on downstream M BONs rather than having an auto-regulatory effect on KCs themselves (Knapek et al., 2013). A more detailed investigation on the $\alpha / \beta-K C$ subtypes revealed distinct functions for these subtypes: $\alpha / \beta_{c}-K C s$ are required for appetitive memory retrieval only and $\alpha / \beta_{s}-K C s$ for both aversive and appetitive memory retrieval (Perisse et al., 2013b). The output of $\alpha^{\prime} / \beta^{\prime}-K C s$, tested with Shit $^{\text {ts }}$ expression, 
showed that this subset is required for appetitive and aversive STM and MTM formation and consolidation, but not retrieval or LTM formation (Cervantes-Sandoval et al., 2013; Krashes et al., $2007)$. The consolidation of memory in $\alpha^{\prime} / \beta^{\prime}-K C s$ works in concord with the MB extrinsic DPM neuron (Cervantes-Sandoval and Davis, 2012; Keene et al., 2004, 2006; Krashes et al., 2007; Waddell et al., 2000; Yu et al., 2005). A structural MB phenotype of the alpha lobe absent (ala) mutant (Boquet et al., 2000), in which randomly either $\alpha / \alpha^{\prime}$ or $\beta / \beta^{\prime}$ are lacking, showed that if $\alpha / \alpha^{\prime}$ but not $\beta / \beta^{\prime}$ are missing, LTM (but not STM, MTM, or ARM) is abolished (Pascual and Preat, 2001).

The role of MBONs

As previously described, the compartment-specific DAN innervation comprises differential memory formation and properties (see this section and 1.4.3). Therefore, it is not surprising that the highly overlapping compartmentalization of MBONs with DANs (see 1.4.3) also exhibits functional differences. Furthermore, given the fact that the above mentioned KC types' output is needed for certain forms of memory formation and memory retrieval (see this section), it is likely that this output affects downstream MBONs. A functional study supported for the first time the assumed model of the valence-dependent behavioral guidance after classical conditioning (Séjourné et al., 2011). This study found that the output of two cholinergic M BONs innervating the vertical lobes in $\alpha 2$ and $\alpha^{\prime} 3$ was required for retrieval of aversive STM, MTM, LTM, and ARM but not acquisition or consolidation (Séjourné et al., 2011). Calcium imaging of those neurons in the same study revealed that their initial naïve odor responses were decreased after conditioning to the odor paired with the electric shock. Another study investigated the role of the MBON innervating $\alpha 3$ (Pai et al., 2013). They found that the output of this MBON is required only for consolidation and retrieval of protein-dependent aversive LTM at a very specific time period after training. On the contrary to the previous study, calcium imaging in this MBON showed an increase of calcium to the CS+presentation when compared to the naïve odor response (Pai et al., 2013). A second study investigating the same MBON confirmed the role of time period-specific LTM retrieval from this MBON additionally in appetitive conditioning and found this MBON to be cholinergic (Plaçais et al., 2013). Calcium imaging of this MBON showed again the increase in calcium transients in response to the CS+ after appetitive conditioning. However, this increase was not just a simple readout of an increased activity in the upstream $\alpha$-KCS as they showed no increase after conditioning, indicating the role of plasticity at the KC-MBON synapse in enhancing M BON responses (Plaçais et al., 2013). The aforementioned studies indicate that memory traces are not just found in KCs themselves but are seemingly transferred to MBONs. 


\section{Introduction}

An extensive study made it its business to investigate the roles of all 34 MBONs in learning and memory via activation with light-activatable CsChrimson or silencing with temperature sensitive Shits (Aso et al., 2014b):

The artificial activation of MBONs can provide insights into their roles in driving approach or avoidance behavior. And indeed, the activation of MBONs elicited approach behavior when induced in some GABAergic and cholinergic MBONs and avoidance behavior when induced in glutamatergic MBONs. Interestingly, the innervation pattern of these MBONs is complementary to each other and contrary in line with DANs conveying punishment or reward, respectively. This means: DANs conveying punishment are connected to MBONs driving approach and the other way around. They further showed that approach or avoidance phenotypes become stronger with the number of co-activated MBON types of the same transmitter type.

The artificial silencing of MBONs can provide insights into the necessities of those MBONs in olfactory learning and memory. In this set of experiments one MBON innervating $\gamma 1$ and the peduncle was found to be required for aversive MTM. This MBON is one of the few projecting its axons back to the MB ( $\alpha$ and $\beta$ ) but also outside of the MB, indicating an information transfer from one lobe to another. Furthermore, this MBON was the only one found to be required for visual aversive memory. For aversive LTM the output of MBONs innervating $\alpha^{\prime} 1, \alpha^{\prime} 3$, and $\alpha 2$ were required.

In appetitive MTM several M BONs were found to be involved innervating e.g., $\gamma^{2}, \alpha^{\prime} 1, \alpha^{\prime} 2, \beta^{\prime} 2, \alpha 1$, and $\alpha 3$. The requirement of MBONs in appetitive visual memory differed slightly from those required in olfactory appetitive memories as they innervate e.g., $\gamma^{4}, \gamma^{5}, \alpha^{\prime} 1, \alpha^{\prime} 3, \beta^{\prime} 2$, and $\alpha 2$. Interestingly the $\gamma 4 \mathrm{MBON}$ projects back to $\gamma 1$ and $\gamma 2$, indicating a role of an MB internal feedforward modulation.

This comprehensive study makes it clear that the orchestration of MBON ensemble output - and presumably not a single MBON - is driving the appropriate behavioral response.

An interesting and striking MB-related switch in behavioral responses after associative conditioning was found in flies where a mild ethanol intoxication was paired with an odor (Kaun et al., 2011). They found that the odor paired with ethanol elicited avoidance on a short term scale but turned into attraction to the odor on a long term scale. The $\mathrm{y}$-lobe was shown to be required for the memory acquisition, $\alpha^{\prime} / \beta^{\prime}-$ lobe for consolidation, and $\alpha / \beta$-lobe for retrieval of long term attraction. The output of a combination of the $\psi$-lobe and $\alpha / \beta$-lobe was required for acquisition and consolidation of short term aversion. These findings indicate the dual role of the $M B$ in opposing behaviors. Later, MBONs innervating $\gamma^{2}, \gamma^{4}, \gamma^{5}, \alpha^{\prime} 1, \alpha^{\prime} 2, \alpha^{\prime} 3$, and $\beta^{\prime} 2$ were shown to be required for the expression of the appetitive long term component (Aso et al., 2014b). 
Subsequent studies concentrated more on smaller sets of MBONs. One of these studies investigated the role of a MBON innervating $\gamma 5$ and $\beta^{\prime} 2$ and showed that this neuron was required for retrieval of MTM, MT-ARM and LT-ARM (Bouzaiane et al., 2015). The MT component was dependent on $\gamma$-lobe output, whereas the LT-ARM component was dependent on $\alpha^{\prime} / \beta^{\prime}$-lobe output. Only simultaneous output blockage of this MBON and another MBON innervating only $\beta^{\prime} 2$ could impair STM.

Another study investigated the plasticity of the MBON innervating $\gamma 1$ and the peduncle (Hige et al., 2015a). They substituted the electric shock by artificially activating a single PPL1- $p 1$ pedc DAN with CsChrimson and paired this activation with odor stimuli (CS+), inducing robust aversive memories. Electrophysiological recordings revealed that the $p 1$ pedc-MBON response to the CS+ was suppressed for several hours after pairing and that this suppression did not arise from suppression in the upstream KCs. However, calcium transients of KCs were measured in the cell body layer and were not $\mathrm{Y}$-KC specific. A later study showed that artificial activation of this M BON with $\mathrm{dTrpA} 1$ could induce appetitive memory, although flies were fed indicating the dual roles of M BONs (Perisse et al., 2016)

A study investigating the role of the $\gamma-\mathrm{KC}$ subtypes $\gamma$ CRE- $p$ and $\gamma$ CRE- $n$ first showed that the M BON innervating $\gamma_{2}$ and $\alpha^{\prime} 1$ was required for appetitive MTM and the MBON innervating $\gamma^{5}$ and $\beta^{\prime} 2$ was required for aversive M TM (Yamazaki et al., 2018). Second, these M BONs were assumed to be putative downstream targets of the two $\gamma-\mathrm{KC}$ subtypes, as silencing of $\gamma 2 \alpha^{\prime} 1-\mathrm{MBON}$ rescued the $\gamma$ CRE-p phenotype (see above, "The role of KC-types") and silencing the $\gamma 5 \beta^{\prime} 2-\mathrm{MBON}$ rescued the $\gamma$ CRE-n phenotype (see above, "The role of KC-types").

The balance of MBONs driving approach or avoidance determines the animal's decision to go towards or away from an odor source. Even though the overall M BON type-specific functions are stereotypic across flies, some MBONs show high experience dependent variability in their odor tuning properties if compared between flies (Hige et al., 2015b). This inter-fly variability (strongest in a2sc-MBONs) was not seen within a fly. Comparing MBONs of both hemispheres in the same animal showed high correlations in their odor tuning properties. This effect was decreased in rut mutants indicating a plasticity and experience-driven mechanism that stabilizes MBON tuning properties within a fly which can therefore be different between flies (Hige et al., 2015b).

In conclusion, the stereotypic and broadly overlapping odor code of the $A L(\approx 50 \mathrm{GL})$ is transformed and expanded into a sparse and highly non-overlapping odor code in KCs $(\approx 2200)$. Coincidence of stimulus-dependent DAN input to KCs and the KC activation due to odor stimulation can form olfactory associative memory traces of different retention times dependent on $\mathrm{KC}$ type and MB compartment. The sparse activity code of $\mathrm{KCS}$ then converges onto a low 
number of broadly tuned MBONs $(\approx 34)$ and is thereby transformed into a valence code in which the ensemble net output of MBONs determines the ultimate behavioral decision.

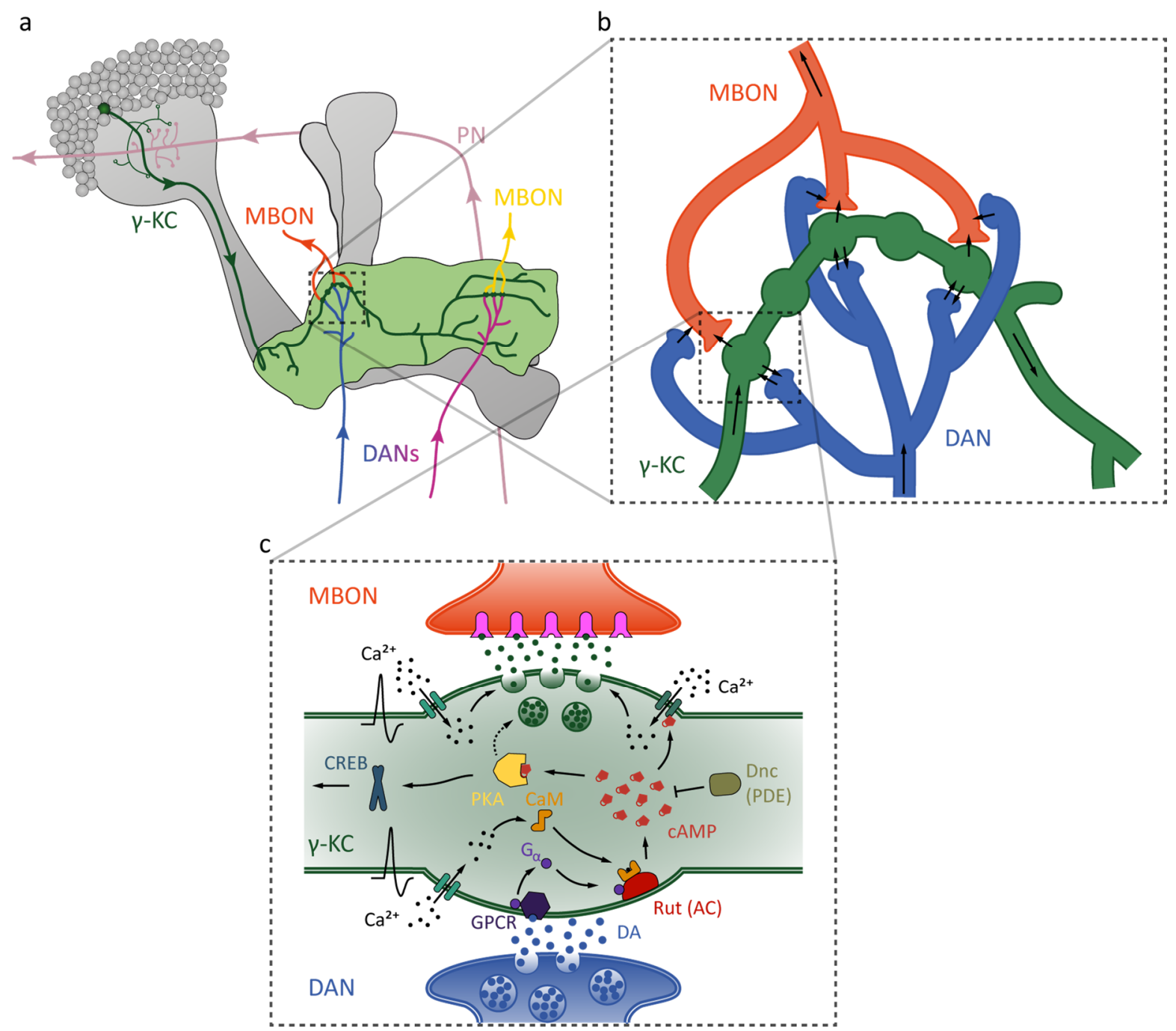

Figure 1.8: Simplified schematic of the information flow and the molecular machinery involved in synaptic plasticity and coincidence detection in the MB circuit of the $\gamma$-lobe. a Olfactory projection neurons convey odor information to $\gamma$-KCs (green) in the calyx of the MB. $\gamma$-KCs project their axons to the lobe region of the MB. DANs convey aversive (blue) and appetitive (purple) stimuli to $\gamma$-KCs compartment specifically. M BONs (orange) take up information conveying it to higher brain centers or partially back to the MB. Dotted rectangle indicates magnified region shown in $\mathbf{b}$. b Simplified connectivity of MB microcircuits. Recent connectome studies (Eichler et al., 2017; Takemura et al., 2017) have shown that DANs are connected to $\gamma^{-}$ $\mathrm{KCS}$ via reciprocal synapses and further form synapses with MBONs. M BONs are further post-synaptic to $\gamma^{-}$ $\mathrm{KC}$ and project to higher brain centers. Dotted rectangle indicates magnified synaptic connectivity shown in c. c Schematic illustration of the molecular pathway involved in olfactory classical conditioning. Voltage gated calcium channels open upon action potential propagation induced by odor stimulation (CS+) leading to $\mathrm{Ca}^{2+}$ influx into the $\mathrm{\gamma}-\mathrm{KC}$. The $\mathrm{Ca}^{2+}$ can bind to calmodulin (CaM). DA release from DANs (US) activates GProtein couple receptors (GPRC) that leads to $G$-protein subunit dissociation $\left(G_{\alpha}\right)$ from the receptor. The coincident activation of $\mathrm{CaM}$ and $\mathrm{G}_{\alpha}$ leads to activation of the adenylate cyclase (AC) Rutabaga (Rut) that is believed to be the coincidence detector mechanism involved classical conditioning. Rut activation leads to an increase of the intracellular CAMP level that can be antagonistically regulated by the phosphodiesterase (PDE) Dunce (Dnc). CAM P has various downstream effects modulating e.g., cyclic nucleotide gate calcium channels that can enhance vesicle release of $y$-KCs on a short term scale. CAMP can also act on protein kinase A (PKA) that can phosphorylate e.g., CAM P response element binding protein (CREB) that alters gene expression or act indirectly on the vesicle release, both inducing long term plastic changes in the presynapse. 
Introduction

\subsection{Further Roles of the MBs}

\subsubsection{PER and Taste Learning}

Olfactory associative learning performances can not only be monitored by the choice of walking into one arm of a T-maze but also by the proboscis extension response (PER), which is altered in the course of aversive (Dejianne et al., 1985; M édioni et al., 1978; Vaysse and M édoni, 1976) and appetitive (Chabaud et al., 2006; Fresquet et al., 1998; Holliday and Hirsch, 1986) learning and involves the rut-dependent CAMP pathway in the MBs (Chabaud et al., 2006). Furthermore, pairing of odors with sweet or bitter tastants involves, self-evidently, taste receptors promoting taste learning that requires the M B output (Masek and Scott, 2010) and the corresponding DANs conveying the taste qualities to the MBs (Burke et al., 2012; Das et al., 2014; Huetteroth et al., 2015).

\subsubsection{Visual Learning}

Besides classical olfactory associative conditioning the MBs are also capable of associating punishment or reward with different visual cues e.g., visual patterns and shapes relative to landmarks or the horizon (Dill et al., 1993; Guo and Götz, 1997), color relative to shapes (Tang and Guo, 2001), colors per se (Aso et al., 2014b; Schnaitmann et al., 2013), or shape orientation, and can be retrieved up to 48 hours after training (Xia et al., 1997). Furthermore, these capabilities allow flies to orient in a visual maze and let them develop spatial memory; however, this kind of learning does not involve the MBs, rather the ellipsoid body and the central complex play an important role (Ofstad et al., 2011). Many of the above mentioned conditioning procedures are accounted to operant conditioning rather than classical conditioning and might be therefore executable without involvement of the MBs (Wolf et al., 1998). However, there are several visual tasks that require the MB circuits, such as context generalization (Liu et al., 1999), decision making in a color-shape dilemma (Tang and Guo, 2001), decision making in color-positioning choices (Zhang et al., 2007a), visual attention (van Swinderen et al., 2009), and associative color conditioning (Aso et al., 2014b; Vogt et al., 2014, 2016). 
Introduction

\subsubsection{Courtship Learning}

Further to olfactory and visual learning, flies can also form a courtship memory that is dependent on previous experience (Siegel and Hall, 1979). Male flies that are placed together with mated female flies experience more rejection than when paired to virgin female flies. This memory of several experienced rejections affects the male flies in subsequent mating experiments, where they then show less courtship to even virgin female flies. This courtship memory involves the MBs because amnesiac learning mutant male flies do not remember the previous rejection periods courting normal virgin female flies afterwards (Siegel and Hall, 1979). Courtship memory usually lasts for approximately 1 hour but can be extended by specific protocols to a 9 day retained LTM (McBride et al., 1999). MB ablation by feeding hydroxyurea (HU) in male flies showed that they have a stronger reduction in memorizing previous rejection periods, being indistinguishable from control flies (no rejection periods) 30 min after training indicating a role of the MBs in courtship memory (McBride et al., 1999). This courtship memory requires the signaling of OA onto $\alpha / \beta-K C S$ expressing the OA receptor OAMB as blockage of their output with $\mathrm{Shi}^{\text {is }}$ reduced the courtship memory performance (Zhou et al., 2012). Furthermore, the $\gamma$-KCs could be shown to be involved in courtship conditioning as the inhibition of $\mathrm{y}$-KC specific expression of the male specific transcription factor Fruitless ${ }^{M}$ (Hall, 1994; Ryner et al., 1996) reduced the conditioning performance (Manoli et al., 2005).

\subsubsection{Roles Besides Learning}

Of course, learning and memory are some of the main roles of the MBs but they comprise also other functions unrelated to learning but of course still influencing learning.

One important aspect of the MB function is the regulation of sleep homeostasis. Sleep in Drosophila (Hendricks et al., 2000; Shaw, 2000) can be altered by manipulation of the MBs e.g., chemical ablation, Shits mediated output block, or increased PKA activity in KCs involving different KC types, especially $\gamma_{d}$ (Joiner et al., 2006; Pitman et al., 2006; Sitaraman et al., 2015). Additionally, sleep can also be altered by the activation of MBONs, where glutamatergic MBONs suppress sleep and GABAergic and cholinergic MBONs promote sleep (Aso et al., 2014b; Sitaraman et al., 2015).

Besides sleep, aggression behavior - especially between male flies (Chen et al., 2002) - was also linked to the MBs as their output (Baier et al., 2002), but also alterations in their volumes and shape, especially $\alpha$-lobes, led to changes in aggression behavior (Edwards et al., 2009; Rollmann et al., 2008). 
The MBs were also shown to be involved in locomotor outputs as ablation experiments revealed an increased duration of walking bouts, but no effect on the number or initiation of these bouts, implying an inhibitory role of the MBs on the walking pattern generator and that MB-defective flies have troubles in terminating walking (M artin et al., 1998).

The manipulation of the PAM -DAN - $\beta / \beta^{\prime}-K C s-\beta / \beta^{\prime}-M B O N$ circuits has been shown to play a role in cold avoidance acting primarily on $\beta^{\prime}-\mathrm{KCs}$ and their downstream MBONs and which is also age dependent (Shih et al., 2015).

\subsection{Scope of the Study}

During the past 42 years many studies have shown that the mushroom bodies are the main site of olfactory associative learning. Although a lot of progress was made in the last decades revealing cellular mechanisms (Kahsai and Zars, 2011) and complex anatomical features (Eichler et al., 2017; Takemura et al., 2017) of the circuit, it is still not fully understood how memory is formed, stored, and ultimately translated into a behavioral response. What was shown is that the $\mathrm{KC}$ intrinsic CAMP pathway, activated and enhanced by the coincident arrival of odor-induced calcium influx and dopamine-induced G-protein activation plays a major role in memory acquisition (see review by Kahsai and Zars, 2011). The activation of its downstream partners stabilizes short term memory components and leads in later stages to de novo protein synthesis, which preserves the formation of long term memory components. The most remarkable anatomical and functional feature of the mushroom body circuit is the overlapping compartmentalized innervation of extrinsic dopaminergic and mushroom body output neurons (Aso et al., 2014a). As this compartmentalization is stereotypic and distinct it is not surprising that aversive or appetitive learning paradigms drive certain MBONs leading to approach or avoidance behavior (Aso et al., 2014b). Interestingly, the compartmentalization of DANs conveying aversive and appetitive stimuli (Aso et al., 2010; Burke et al., 2012; Claridge-Chang et al., 2009; Cohn et al., 2015; Liu et al., 2012; Riemensperger et al., 2005) applies not to specific KCs that only arbor into those compartments. Instead, distinct DANs innervate the whole axon of the same KC. This implies that both aversive and appetitive memories are formed and stored in different regions of the cell, functionally uncoupling branches of the same neuron. So far, functional imaging studies always only investigated the whole KC population (Akalal et al., 2010; Barth et al., 2014; Cohn et al., 2015; Wang et al., 2008; Yu et al., 2006), and single cell studies focused only on KC somata or calical micro glomeruli (Gruntman and Turner, 2013; Honegger et al., 2011; Pech et al., 2015). As DANs and MBONs implicated in associative learning innervate the axonal regions, the 


\section{Introduction}

investigation of the cell somata or calices do not necessarily represent learning-induced changes in the lobes. In terms of learning studies, the whole cell population imaging furthermore lacks single cell resolution that also prevents the investigation of single synapses that are thought to undergo plasticity in the course of learning. So far it is not fully understood how KC synapses change their activity in the course of olfactory associative conditioning on a short term scale. This refers to the first criterion of defining a memory trace: that there has to be synaptic plasticity in the neuronal substrate (Gerber et al., 2004; Martin et al., 2000; Thompson, 2005). Furthermore, the sparse coding of the KC population (Honegger et al., 2011; Turner et al., 2008) has so far hampered the detection of memory traces in KCs.

In this study, I aimed to tackle this task by monitoring calcium transients in single KCs of the Drosophila MB and investigate synaptic plasticity on a short term scale. Drosophila has an elaborate olfactory system and performs well in olfactory associative conditioning (Tully and Quinn, 1985), allowing for a detailed investigation of memory traces. In addition to that, the versatile set of genetic tools enabling the monitoring of synaptic plasticity prompted me to use Drosophila as a model organism to bring our insight into associative learning forward. In order to investigate associative learning and memory at the single cell level I used the M ARCM technique (Lee and Luo, 1999), which randomly labels one or a few KCs. I aimed at investigating short term memory and therefore restricted the expression to the $y$-lobe, which was shown to be mainly involved in short term memory (Akalal et al., 2006; Blum et al., 2009; Zars et al., 2000). Studies that investigate learning and memory on a physiological level by using calcium imaging usually train flies in learning chambers (Tully and Quinn, 1985) and later subjected them to the imaging procedure (Akalal et al., 2010, 2011; Wang et al., 2008). This procedure allows only for calcium imaging after at least 10 to $15 \mathrm{~min}$ after testing (time for handling and preparation) and precedes anesthesia and preparation stress, which might influence the brain physiology and therefore learning induced changes. In my study, I trained the flies under the microscope, allowing me to monitor the immediate short term changes after associative training without any further disturbance of memory consolidation, which would not be possible if trained outside. 


\section{Materials and Methods}

\subsection{Materials}

\subsubsection{Drosophila Fly Strains}

The following table lists the parental fly strains that were used in this study. For fly strains generated through combinatorial crosses and used for experiments see 2.2.1:

\begin{tabular}{|c|c|c|}
\hline Genotype & Donor & Reference \\
\hline y1, w', FRT19A; ; & Bloomington (\#1744) & Xu and Rubin, 1993 \\
\hline $\begin{array}{l}\text { hsFLP, tubP-Gal80, w-, neoFRT19A; } \\
\text {; }\end{array}$ & Bloomington (\#5132) & $\begin{array}{l}\text { Golic, 1991; Lee and Luo, } \\
\text { 1999; Xu and Rubin,1993 }\end{array}$ \\
\hline 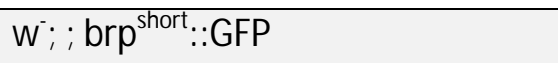 & Stephan Sigrist & Fouquet et al., 2009 \\
\hline $\begin{array}{l}\text { y, w; Sp/CyO, Wee-P; 20XUAS- } \\
6 \text { XmCherry }\end{array}$ & Bloomington (\#52268) & Shearin et al., 2014 \\
\hline$w^{\circ} ; ; 5 H T 1 B-G A L 4$ & Bloomington (\#27637) & Yuan et al., 2005 \\
\hline w;; ; 20XUAS-GCaMP3 & Bloomington (\#32237) & Tian et al., 2009 \\
\hline W־; 20XUAS-GCaM P3; & Toshihara & Tian et al., 2009 \\
\hline
\end{tabular}

\subsubsection{Fly Food}

The following table lists the ingredients for 20 I standard cornmeal food that was used throughout this study:

\begin{tabular}{|l|l|l|}
\hline Ingredient (quantity) & Company & Address \\
\hline thread agar (205 g) & Gourvita GmbH & $\begin{array}{l}\text { Adam-Opel-Str. 19 } \\
\text { D-63322 Rödermark }\end{array}$ \\
\hline soy flour $(200 \mathrm{~g})$ & $\begin{array}{l}\text { Pflanzensaftwerk GmbH \& Co. } \\
\text { KG }\end{array}$ & $\begin{array}{l}\text { Hutwiesenstraße 14 } \\
\text { D-71106 Magstadt }\end{array}$ \\
\hline brewer's yeast $(360 \mathrm{~g})$ & Gourvita GmbH & $\begin{array}{l}\text { Adam-Opel-Str. 19 } \\
\text { D-63322 Rödermark }\end{array}$ \\
\hline
\end{tabular}




\begin{tabular}{|l|l|l|}
\hline cornmeal $(1600 \mathrm{~g})$ & ZlELER \& CO. GmbH & $\begin{array}{l}\text { Liebigstraße 101 } \\
\text { D-22113 Hamburg }\end{array}$ \\
\hline sugar beet syrup $(440 \mathrm{~g})$ & Obermühle Rosdorf & $\begin{array}{l}\text { Obere M ühlenstraße 3 } \\
\text { D-37124 Rosdorf }\end{array}$ \\
\hline malt $(1600 \mathrm{~g})$ & MeisterM arken - Ulmer Spatz & $\begin{array}{l}\text { M ainzer Straße 152-160 } \\
\text { D-55411 Bingen am Rhein }\end{array}$ \\
\hline propionic acid $(126 \mathrm{ml})$ & Carl Roth GmbH + Co. KG & $\begin{array}{l}\text { Schoemperlenstr. 1-5 } \\
\text { D-76185 Karlsruhe }\end{array}$ \\
\hline nipagin (30 g) & Sigma-Aldrich Chemie GmbH & $\begin{array}{l}\text { Eschenstraße 5 } \\
\text { D-82024 Taufkirchen }\end{array}$ \\
\hline ethanol $(140 \mathrm{ml})$ & VWR International GmbH & $\begin{array}{l}\text { Hilpertstraße 20a } \\
\text { D-64295 Darmstadt }\end{array}$ \\
\hline
\end{tabular}

\subsubsection{Chemicals}

\begin{tabular}{|c|c|c|}
\hline Name & Company & Address \\
\hline $\mathrm{KCl}$ & Carl Roth GmbH +Co. KG & $\begin{array}{l}\text { Schoemperlenstr. 1-5 } \\
\text { D-76185 Karlsruhe }\end{array}$ \\
\hline $\mathrm{NaCl}$ & AppliChem GmbH & $\begin{array}{l}\text { Ottoweg } 4 \\
\text { D-64291 Darmstadt }\end{array}$ \\
\hline $\mathrm{MgCl}_{2}$ & Carl Roth $\mathrm{GmbH}+\mathrm{Co} . \mathrm{KG}$ & $\begin{array}{l}\text { Schoemperlenstr. 1-5 } \\
\text { D-76185 Karlsruhe }\end{array}$ \\
\hline $\mathrm{CaCl}_{2}$ & Carl Roth $\mathrm{GmbH}+\mathrm{Co} . \mathrm{KG}$ & $\begin{array}{l}\text { Schoemperlenstr. 1-5 } \\
\text { D-76185 Karlsruhe }\end{array}$ \\
\hline Hepes & Carl Roth GmbH +Co. KG & $\begin{array}{l}\text { Schoemperlenstr. 1-5 } \\
\text { D-76185 Karlsruhe }\end{array}$ \\
\hline sucrose & Carl Roth $\mathrm{GmbH}+\mathrm{Co} . \mathrm{KG}$ & $\begin{array}{l}\text { Schoemperlenstr. 1-5 } \\
\text { D-76185 Karlsruhe }\end{array}$ \\
\hline $\mathrm{NaH}_{2} \mathrm{PO}_{4}$ & Carl Roth GmbH +Co. KG & $\begin{array}{l}\text { Schoemperlenstr. 1-5 } \\
\text { D-76185 Karlsruhe }\end{array}$ \\
\hline $\mathrm{Na}_{2} \mathrm{HPO}_{4}$ & Carl Roth GmbH +Co. KG & $\begin{array}{l}\text { Schoemperlenstr. 1-5 } \\
\text { D-76185 Karlsruhe }\end{array}$ \\
\hline Triton X 100 & Carl Roth $\mathrm{GmbH}+\mathrm{Co} . \mathrm{KG}$ & $\begin{array}{l}\text { Schoemperlenstr. 1-5 } \\
\text { D-76185 Karlsruhe }\end{array}$ \\
\hline
\end{tabular}




\begin{tabular}{|l|l|l|}
\hline $\begin{array}{l}\text { Albumin Fraktion V } \\
\text { (bovine serum albumin - BSA) }\end{array}$ & Carl Roth GmbH +Co. KG & $\begin{array}{l}\text { Schoemperlenstr. 1-5 } \\
\text { D-76185 Karlsruhe }\end{array}$ \\
\hline paraformaldehyde & Carl Roth $\mathrm{GmbH}+$ Co. KG & $\begin{array}{l}\text { Schoemperlenstr. 1-5 } \\
\text { D-76185 Karlsruhe }\end{array}$ \\
\hline $\mathrm{HCl}$ & Carl Roth GmbH +Co. KG & $\begin{array}{l}\text { Schoemperlenstr. 1-5 } \\
\text { D-76185 Karlsruhe }\end{array}$ \\
\hline $\mathrm{NaOH}$ & Carl Roth GmbH +Co. KG & $\begin{array}{l}\text { Schoemperlenstr. 1-5 } \\
\text { D-76185 Karlsruhe }\end{array}$ \\
\hline
\end{tabular}

\subsubsection{Consumables}

\begin{tabular}{|c|c|c|}
\hline Name & Company & Address \\
\hline KENTOFLOW (UV-glue) & Kent Express Limited & $\begin{array}{l}\text { Unit 9, Studland Road } \\
\text { U.K.-NN2 6NE Northhampton }\end{array}$ \\
\hline pipette tips & Sarstedt AG \& Co & $\begin{array}{l}\text { Sarstedtstraße } 1 \\
\text { D-51588 Nümbrecht }\end{array}$ \\
\hline $\begin{array}{l}\text { cover glasses } \\
18 \mathrm{~mm} \times 18 \mathrm{~mm}\end{array}$ & Thermo Fisher Scientific GmbH & $\begin{array}{l}\text { Im Steingrund 4-6 } \\
\text { D-63303 Dreieich }\end{array}$ \\
\hline $\begin{array}{l}\text { cover glasses } \\
24 \mathrm{~mm} \times 60 \mathrm{~mm}\end{array}$ & Th. Geyer GmbH \& Co. KG & $\begin{array}{l}\text { Dornierstr. 4-6 } \\
\text { D-71272 Renningen }\end{array}$ \\
\hline microscope slides & Carl Roth $\mathrm{GmbH}+\mathrm{Co} . \mathrm{KG}$ & $\begin{array}{l}\text { Schoemperlenstr. 1-5 } \\
\text { D-76185 Karlsruhe }\end{array}$ \\
\hline $\begin{array}{l}\text { Austerlitz INSECT PINS (0.1 } \\
\mathrm{mm})\end{array}$ & Pin Service - Lucie Hrabovská & $\begin{array}{l}\text { Čsl. Červeného kříže } 967 \\
\text { CZE-68401 Slavkov u Brna }\end{array}$ \\
\hline transparent tape rings & Avery Zweckform GmbH & $\begin{array}{l}\text { M iesbacher Str. } 5 \\
\text { D-83626 Oberlaindern }\end{array}$ \\
\hline $\begin{array}{l}\text { VECTASHIELD (mounting } \\
\text { medium) }\end{array}$ & Vector Laboratories, Inc. & $\begin{array}{l}30 \text { Ingold Road } \\
\text { USA-Burlingame, Ca } 94010\end{array}$ \\
\hline transparent nail polish & L'Oréal International & $\begin{array}{l}\text { 41, Rue Martre } \\
\text { F-92117 Clichy Cedex }\end{array}$ \\
\hline forceps & Fine Science Tools GmbH & $\begin{array}{l}\text { Vangerowstraße } 14 \\
\text { D-69115 Heidelberg }\end{array}$ \\
\hline food vials & Sarstedt AG \& Co & $\begin{array}{l}\text { Sarstedtstraße } 1 \\
\text { D-51588 Nümbrecht }\end{array}$ \\
\hline
\end{tabular}




\begin{tabular}{|l|l|l|}
\hline stab knife (5 mm blade) & Sharpoint & $\begin{array}{l}\text { 1100 Berkshire Blvd \#308 } \\
\text { USA-Wyomissing, PA } 19610\end{array}$ \\
\hline surgical disposable scalpel (11) & BRAUN - Aesculap AG & $\begin{array}{l}\text { Am Aesculap-Platz } \\
\text { D-78532 Tuttlingen }\end{array}$ \\
\hline $\begin{array}{l}\text { hypodermic-needle (1.1 x 50 } \\
\text { mm) }\end{array}$ & $\begin{array}{l}\text { Sterican - B. Braun M elsungen } \\
\text { AG }\end{array}$ & $\begin{array}{l}\text { Carl-Braun-Straße 1 } \\
\text { D-34212 M elsungen }\end{array}$ \\
\hline scintillation vial (20 ml) & Sarstedt AG \& Co & $\begin{array}{l}\text { Sarstedtstraße 1 } \\
\text { D-51588 Nümbrecht }\end{array}$ \\
\hline
\end{tabular}

\subsubsection{Solutions and Buffers}

\begin{tabular}{|c|c|c|}
\hline Name & Ingredient & Comment \\
\hline Ringer's solution & $\begin{array}{l}5 \mathrm{mM} \mathrm{KCl} \\
130 \mathrm{mM} \mathrm{NaCl} \\
2 \mathrm{mM} \mathrm{M} \mathrm{gCl} 2 * 2 \mathrm{H}_{2} \mathrm{O} \\
2 \mathrm{mM} \mathrm{CaCl}_{2} \\
5 \mathrm{mM} \text { Hepes } \\
36 \mathrm{mM} \text { sucrose }\end{array}$ & $\begin{array}{l}\mathrm{pH} 7.3 \text { (adjusted with } \mathrm{HCl} \text { or } \\
\mathrm{NaOH} \text { ) } \\
\text { stored at }-20^{\circ} \mathrm{C} \\
\text { after use at } 4{ }^{\circ} \mathrm{C}\end{array}$ \\
\hline $\begin{array}{l}\text { PBS (phosphate buffered } \\
\text { saline) }\end{array}$ & $\begin{array}{l}15 \mathrm{mM} \mathrm{NaH}_{2} \mathrm{PO}_{4} \\
100 \mathrm{mM} \mathrm{NaCl} \\
85 \mathrm{mM} \mathrm{Na}_{2} \mathrm{HPO}_{4}\end{array}$ & $\begin{array}{l}\mathrm{pH} 7.4 \text { (adjusted with } \mathrm{HCl} \text { or } \\
\mathrm{NaOH} \text { ) } \\
\text { stored at } 4{ }^{\circ} \mathrm{C}\end{array}$ \\
\hline PBST (PBS + Triton X 100) & $\begin{array}{l}\text { PBS } \\
0.6 \% \text { Triton } \times 100\end{array}$ & stored at $4{ }^{\circ} \mathrm{C}$ \\
\hline blocking solution & $\begin{array}{l}\text { PBST } \\
2 \% \text { bovine serum albumin }\end{array}$ & stored at $4{ }^{\circ} \mathrm{C}$ \\
\hline PFA (paraformaldehyde) & $\begin{array}{l}\text { PBS } \\
4 \% \text { paraformaldehyde } \\
0.1 \% \mathrm{NaOH}\end{array}$ & $\begin{array}{l}\text { pH } 7.4 \text { (adjusted with } \mathrm{HCl} \text { or } \\
\mathrm{NaOH} \text { ) } \\
\text { ingredients are mixed at } 70^{\circ} \mathrm{C} \\
\text { and pH adjusted at } 20^{\circ} \mathrm{C} \\
\text { stored at }-20^{\circ} \mathrm{C}\end{array}$ \\
\hline
\end{tabular}




\subsubsection{Odors/Solvent}

\begin{tabular}{|l|l|l|}
\hline Name & Company & Address \\
\hline mineral oil (M 8410) & SIGM A-ALDRICH & $\begin{array}{l}\text { 3050 Spruce St. } \\
\text { USA- St. Louis, M O } 63103\end{array}$ \\
\hline $\begin{array}{l}\text { 4-M ethylcyclohexanol (MCH - } \\
\text { 1:750, 153095) }\end{array}$ & SIGM A-ALDRICH & $\begin{array}{l}\text { 3050 Spruce St. } \\
\text { USA- St. Louis, M O } 63103\end{array}$ \\
\hline $\begin{array}{l}\text { 3-Octanol (3-Oct - 1:500, } \\
\text { 1-Octanol (1-Oct - 1:400, } \\
\text { 297887) }\end{array}$ & SIGM A-ALDRICH & $\begin{array}{l}\text { 3050 Spruce St. } \\
\text { USA- St. Louis, M O } 63103\end{array}$ \\
\hline
\end{tabular}

\subsubsection{Antibodies}

\begin{tabular}{|c|c|c|c|c|c|}
\hline & Antigen & $\begin{array}{l}\text { Raise } \\
\text { d in }\end{array}$ & Fluorophore & $\begin{array}{l}\text { Concentration } \\
\text { used }\end{array}$ & Source, catalogue \# \\
\hline \multirow[t]{2}{*}{$\begin{array}{l}\text { primary } \\
\text { antibodies }\end{array}$} & DLG - discs large & $\begin{array}{l}\text { mous } \\
\mathrm{e}\end{array}$ & - & $1: 200$ & $\begin{array}{l}\text { Developmental } \\
\text { Studies Hybridoma } \\
\text { Bank, 4F3 }\end{array}$ \\
\hline & $\begin{array}{l}\text { GFP - green } \\
\text { fluorescent } \\
\text { protein }\end{array}$ & rabbit & - & $1: 2000$ & Invitrogen, A6455 \\
\hline \multirow[t]{2}{*}{$\begin{array}{l}\text { secondary } \\
\text { antibodies }\end{array}$} & mouse - IgG & goat & $\begin{array}{l}\text { Alexa Fluor } \\
633\end{array}$ & $1: 300$ & Invitrogen, A21050 \\
\hline & rabbit - IgG & goat & $\begin{array}{l}\text { Alexa Fluor } \\
488\end{array}$ & $1: 300$ & $\begin{array}{l}\text { Life Technologies, } \\
\text { A11034 }\end{array}$ \\
\hline
\end{tabular}

\subsubsection{Microscopy Equipment}

2-Photon microscopy:

\begin{tabular}{|l|l|l|}
\hline Name & Company & Address \\
\hline LSM 7MP & Carl Zeiss AG & $\begin{array}{l}\text { Carl-Zeiss-Straße 22 } \\
\text { D-73447 Oberkochen }\end{array}$ \\
\hline mode-locked Ti-sapphire laser & Coherent Inc. & 5100 Patrick Henry Drive \\
\hline
\end{tabular}




\begin{tabular}{|l|l|l|}
\hline & & USA- Santa Clara, CA 95054 \\
\hline $\begin{array}{l}\text { dichroic mirror (500-550/650- } \\
660 \mathrm{~nm} \text { BP-filter) }\end{array}$ & Carl Zeiss AG & $\begin{array}{l}\text { Carl-Zeiss-Straße 22 } \\
\text { D-73447 Oberkochen }\end{array}$ \\
\hline $\begin{array}{l}\text { plan-Apochromat 20x (NA =1) } \\
\text { water immersion objective }\end{array}$ & Carl Zeiss AG & $\begin{array}{l}\text { Carl-Zeiss-Straße 22 } \\
\text { D-73447 Oberkochen }\end{array}$ \\
\hline
\end{tabular}

Confocal microscopy:

\begin{tabular}{|l|l|l|}
\hline Name & Company & Address \\
\hline $\begin{array}{l}\text { TSC SP8 confocal laser } \\
\text { scanning microscope }\end{array}$ & Leica M icrosystems GmbH & $\begin{array}{l}\text { Ernst-Leitz-Straße 17-37 } \\
\text { D-35578 Wetzlar }\end{array}$ \\
\hline $\begin{array}{l}\text { PL FLUOTAR 10x (NA =0.3) air } \\
\text { objective }\end{array}$ & Leica M icrosystems GmbH & $\begin{array}{l}\text { Ernst-Leitz-Straße 17-37 } \\
\text { D-35578 Wetzlar }\end{array}$ \\
\hline $\begin{array}{l}\text { PL APO 20x (NA =0.75) } \\
\text { glycerol/water objective }\end{array}$ & Leica M icrosystems GmbH & $\begin{array}{l}\text { Ernst-Leitz-Straße 17-37 } \\
\text { D-35578 Wetzlar }\end{array}$ \\
\hline Argon-laser (488 nm) & Leica M icrosystems GmbH & $\begin{array}{l}\text { Ernst-Leitz-Straße 17-37 } \\
\text { D-35578 Wetzlar }\end{array}$ \\
\hline DPSS-laser $(561 \mathrm{~nm})$ & Leica M icrosystems GmbH & $\begin{array}{l}\text { Ernst-Leitz-Straße 17-37 } \\
\text { D-35578 Wetzlar }\end{array}$ \\
\hline HeNe-laser $(633 \mathrm{~nm})$ & Leica M icrosystems GmbH & $\begin{array}{l}\text { Ernst-Leitz-Straße 17-37 } \\
\text { D-35578 Wetzlar }\end{array}$ \\
\hline
\end{tabular}

\subsubsection{Software}

\begin{tabular}{|l|l|l|}
\hline Name & Company & Address \\
\hline $\begin{array}{l}\text { M icrosoft Office 2010 (Excel, } \\
\text { Word, PowerPoint) }\end{array}$ & M icrosoft Corporation & $\begin{array}{l}\text { One M icrosoft Way } \\
\text { USA-Redmond, WA } 98052\end{array}$ \\
\hline Image) & National Institutes of Health & $\begin{array}{l}9000 \text { Rockville Pike } \\
\text { USA-Bethesda, M aryland } \\
20892\end{array}$ \\
\hline OriginPro 8.5G & OriginLab Corporation & $\begin{array}{l}\text { One Roundhouse Plaza } \\
\text { USA-Northampton, M A 01060 }\end{array}$ \\
\hline Adobe IIlustrator CS6 & Adobe System Incorporated & $\begin{array}{l}345 \text { Park Avenue } \\
\text { USA-San Jose, CA 95110-2704 }\end{array}$ \\
\hline ZEN 2011 SP2 & Carl Zeiss AG & Carl-Zeiss-Straße 22 \\
\hline
\end{tabular}




\begin{tabular}{|c|c|c|}
\hline & & D-73447 Oberkochen \\
\hline Leica Application Suite X (LAS) & Leica M icrosystems GmbH & $\begin{array}{l}\text { Ernst-Leitz-Straße 17-37 } \\
\text { D-35578 Wetzlar }\end{array}$ \\
\hline M ATLAB (R2012b) & MathWorks & $\begin{array}{l}1 \text { Apple Hill Drive } \\
\text { USA-Natick, MA 01760-2098 }\end{array}$ \\
\hline Zotero 5.0 .44 & $\begin{array}{l}\text { Roy Rosenzweig Center for } \\
\text { History and New M edia - } \\
\text { George M ason University }\end{array}$ & $\begin{array}{l}4400 \text { University Dr } \\
\text { USA-Fairfax, VA } 22030\end{array}$ \\
\hline $\begin{array}{l}\text { GraphPad Prism } 7.04 \text { (Trial } \\
\text { Version) }\end{array}$ & GraphPad Software & $\begin{array}{l}7825 \text { Fay Avenue, Suite } 230 \\
\text { La Jolla } \\
\text { USA. CA } 92037\end{array}$ \\
\hline
\end{tabular}

\subsection{Methods}

\subsubsection{Generation of Fly Strains for M ARCM Experiments}

All flies were kept on standard cornmeal food at $25^{\circ} \mathrm{C}$ and $60 \%$ humidity under a $12 / 12 \mathrm{~h}$ lightdark cycle.

The following fly strains were provided by the lab and derived from the initial fly strains mentioned above (2.1.1) and used for further crossing (see section below the parental strains):

Parental strains:

$P_{1} \frac{h s F l p, t u b P-G A L 80, w-, \text { neoFRT19A }}{h s F l p, t u b P-G A L 80, w-, \text { neoFRT19A }} ; \frac{\text { Gla }}{\text { Cyo }} ; \frac{+}{+}$

$P_{2} \frac{h s F l p, t u b P-G A L 80, w-, \text { neoFRT } 19 A}{h s F l p, t u b P-G A L 80, w-, \text { neoFRT } 19 A} ; \frac{+}{+} ; \frac{T M 3}{T M 6}$

$P_{3} \frac{h s F l p, t u b P-G A L 80, w-, \text { neoFRT } 19 A}{h s F l p, t u b P-G A L 80, w-, \text { neoFRT } 19 A} ; \frac{20 X U A S-6 X m \text { Cherry }}{C y O} ; \frac{+}{+}$

$P_{4} \frac{h s F l p, t u b P-G A L 80, w-, \text { neoFRT } 19 A}{h s F l p, t u b P-G A L 80, w-, \text { neoFRT } 19 A} ; \frac{+}{+} ; \frac{20 x U A S-G C a M P 3}{T M 3}$

$P_{5} \frac{y 1, w-, \text { neoF } R T 19 A}{y 1, w-, \text { neoFRT } 19 A} ; \frac{ \pm}{+} ; \frac{5 H T 1 B-G A L 4}{5 H T 1 B-G A L 4}$

$P_{6} \frac{h s F l p, t u b P-G A L 80, w-, \text { neoFRT19A }}{\text { hsFlp,tubP-GAL80,w-,neoFRT19A }} ; \frac{20 X U A S-6 X m C h e r r y}{C y O} ; \frac{U A S-b r p^{\text {short }}:: G F P}{T M 6}$ 
The parental fly strains $P_{1}-P_{4}$ and $P_{6}$ carried hsFlp, tubP-GAL80, neoFRT19A on the first chromosome that are one part necessary for the MARCM-single cell clone system. $P_{5}$ carried only neoFRT19A on the first chromosome as well that is the second part for this system. Both parts had to be combined to induce expression in single KCs (see below). $P_{1}$ and $P_{2}$ carried two balancers on either the second or the third chromosome to prevent unintended recombination events. $P_{3}$ carried the red fluorescence protein mCherry that was used as a cell tracer. $\mathrm{P}_{4}$ carries the $\mathrm{Ca}^{2+}$-indicator GCaMP3 to monitor the activity of the KCs. $\mathrm{P}_{5}$ carried the 5HT1B-GAL4 that mainly restricts the expression of proteins of interest to the $\mathrm{y}$-lobe KCs. $\mathrm{P}_{6}$ carries mCherry for cell tracing and a GFP-construct that is tagged to the pre-synaptic protein Bruchpilot (BRP) to label the pre-synapses of single KCs with GFP.

Generation of MARCM fly strain for 2-photon imaging:

$\mathrm{P}_{1} \times \mathrm{P}_{2}$

$\Varangle \frac{h s F l p, t u b P-G A L 80, w-, \text { neoFRT19A }}{h S F l p, t u b P-G A L 80, w-, \text { neoFRT } 19 A} ; \frac{G l a}{C y o} ; \frac{+}{+}$

$\times$

§ $\frac{h S F l p, t u b P-G A L 80, w-, \text { neoFRT } 19 A}{Y} ; \frac{+}{+} ; \frac{T M 3}{T M 6}$

$\Rightarrow \mathrm{F}_{1} \frac{h s F l p, t u b P-G A L 80, w-, n e o F R T 19 A}{Y} ; \frac{G l a}{+} ; \frac{+}{T M 6}$

$\Rightarrow \mathrm{F}_{2} \frac{h s F l p, t u b P-G A L 80, w-, \text { neoFRT19A }}{Y} ; \frac{+}{C y O} ; \frac{T M 3}{+}$

$\mathrm{P}_{3} \times \mathrm{F}_{1}$

市 $\frac{\text { hsFlp,tubP-GAL80,w-,neoFRT19A }}{\text { hsFlp,tubP-GAL80,w-,neoFRT19A }} ; \frac{20 X U A S-6 \text { XmCherry }}{\text { CyO }} ; \frac{+}{+}$

$\times$

§ $\frac{h s F l p, t u b P-G A L 80, w-, \text { neoFRT } 19 A}{Y} ; \frac{\text { Gla }}{+} ; \frac{+}{T M 6}$

$\Rightarrow \mathrm{F}_{3} \frac{h s F l p, t u b P-G A L 80, w-, \text { neoFRT19A }}{h s F l p, t u b P-G A L 80, w-, \text { neoFRT } 19 A} ; \frac{20 X U A S-6 \text { XmCherry }}{\text { Gla }} ; \frac{+}{T M 6}$

$\mathrm{P}_{4} \times \mathrm{F}_{2}$

$\Varangle \frac{h s F l p, t u b P-G A L 80, w-, \text { neoFRT19A }}{h s F l p, t u b P-G a A L 0, w-, \text { neoFRT } 19 A} ; \frac{+}{+} ; \frac{20 x U A S-G C a M P 3}{T M 3}$

$\times$

§. $\frac{h s F l p, t u b P-G A L 80, w-, n e o F R T 19 A}{Y} ; \frac{+}{C y O} ; \frac{T M 3}{+}$ 


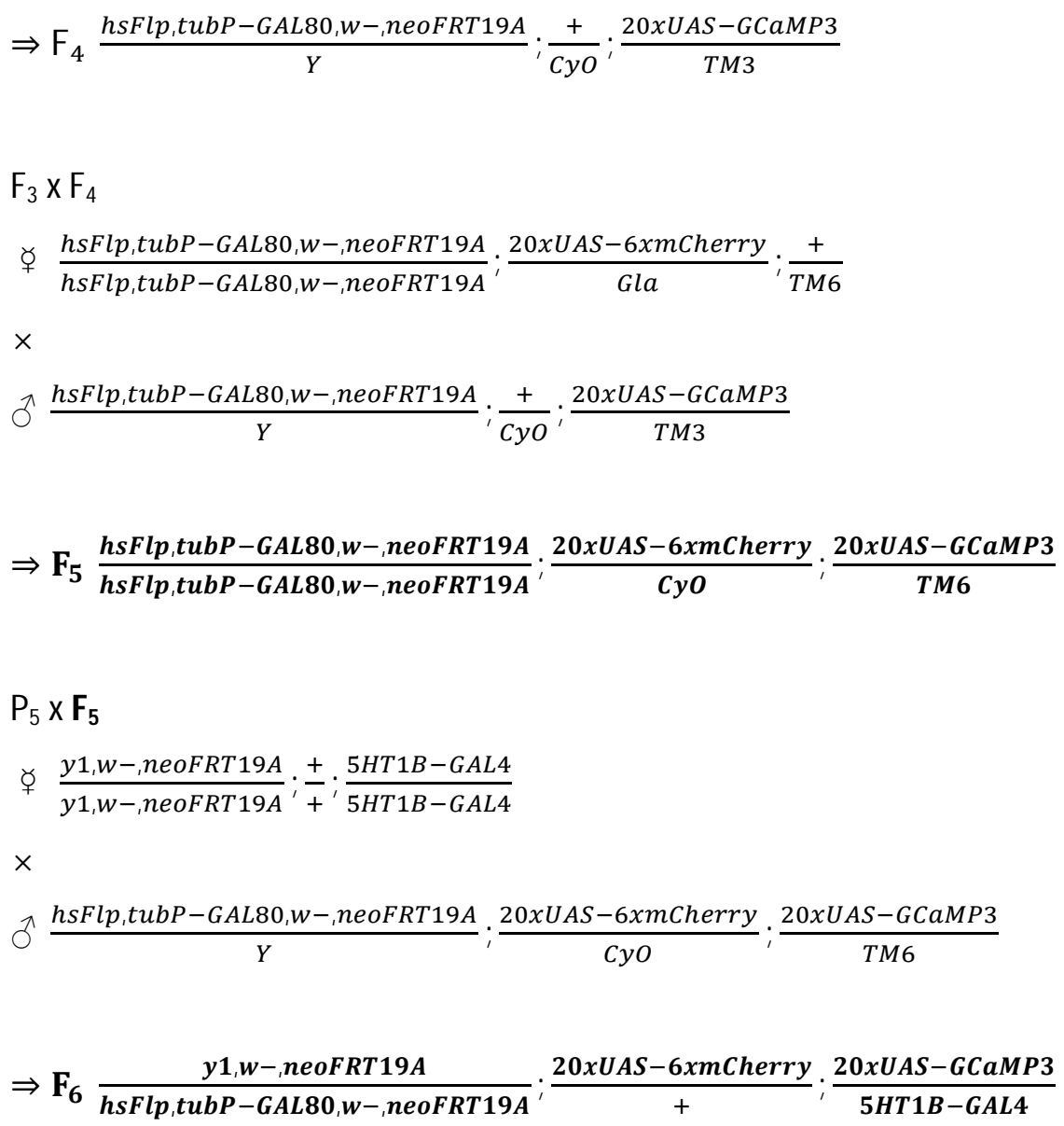

$\mathbf{F}_{5}$ carried balancers on the 2 nd and $3 r$ chromosome to prevent recombination events and is therefore considered a stable fly strain that was used as parental strain (highlighted in bold letters) for follow up crosses. $\mathbf{F}_{6}$ was the fly strain of which female flies were used for 2 -photon imaging experiments (highlighted in bold letters) to investigate the odor responses and the effect of olfactory associative learning in single $\mathrm{\gamma}$-KCs. As it did not carry balancers and unintended recombination could have taken place it was considered as an unstable fly strain that had to be crossed $\left(P_{5} \times F_{5}\right)$ constantly throughout the study.

Generation of MARCM fly strain for confocal imaging:

$\mathrm{P}_{5} \times \mathrm{P}_{6}$

ఫ $\frac{y 1, w-, \text { neoFRT } 19 A}{y 1, w-, \text { neoFRT } 19 A} ; \frac{+}{+} ; \frac{5 H T 1 B-G A L 4}{5 H T 1 B-G A L 4}$

$\times$

○ी $\frac{\text { hsFlp,tubP-GAL80,w-,neoFRT19A }}{Y} ; \frac{20 X U A S-6 \text { XmCherry }}{\text { CyO }} ; \frac{U A S-\text { brp }{ }^{\text {short }}:: G F P}{\text { TM6 }}$

$\Rightarrow \mathrm{F}_{7} \frac{y 1, w-, \text { neoFRT19A }}{\text { hsFlp,tubP-GAL80,w-,neoFRT19A }} ; \frac{20 x U A S-6 x m C h e r r y}{+} ; \frac{U A S-\text { brp }^{\text {short }}:: G F P}{5 H T 1 B-G A L 4}$ 
F7 was the fly strain of which female flies were used for confocal imaging experiments (highlighted in bold letters) to investigate the co-localization of the pre-synaptic protein Bruchpilot with synaptic boutons in single KCs. However, it was an unstable fly strain as well and had to be crossed $\left(P_{5} \times P_{6}\right)$ constantly.

\subsubsection{Generation of Single Cell Clones with the M ARCM-Technique}

Parental fly strains $\left(P_{5} \times F_{5}\right.$ and $\left.P_{5} \times P_{6}\right)$ were crossed and allowed to lay eggs for $4 \mathrm{~h}$. $48 \mathrm{~h}$ later the larvae were exposed to a heat shock (Fig. 2.1) in a $37{ }^{\circ} \mathrm{C}$ water bath for $2 \times 45 \mathrm{~min}$ with a $30 \mathrm{~min}$ break in between to induce FLP activity during the development of $y$-lobe KCs (Lee et al., 1999). As the MARCM -technique requires heterozygous GAL80 expression (see 1.2.2 and 2.2.1) on the first chromosome, only female flies could be used for calcium imaging and confocal imaging experiments.
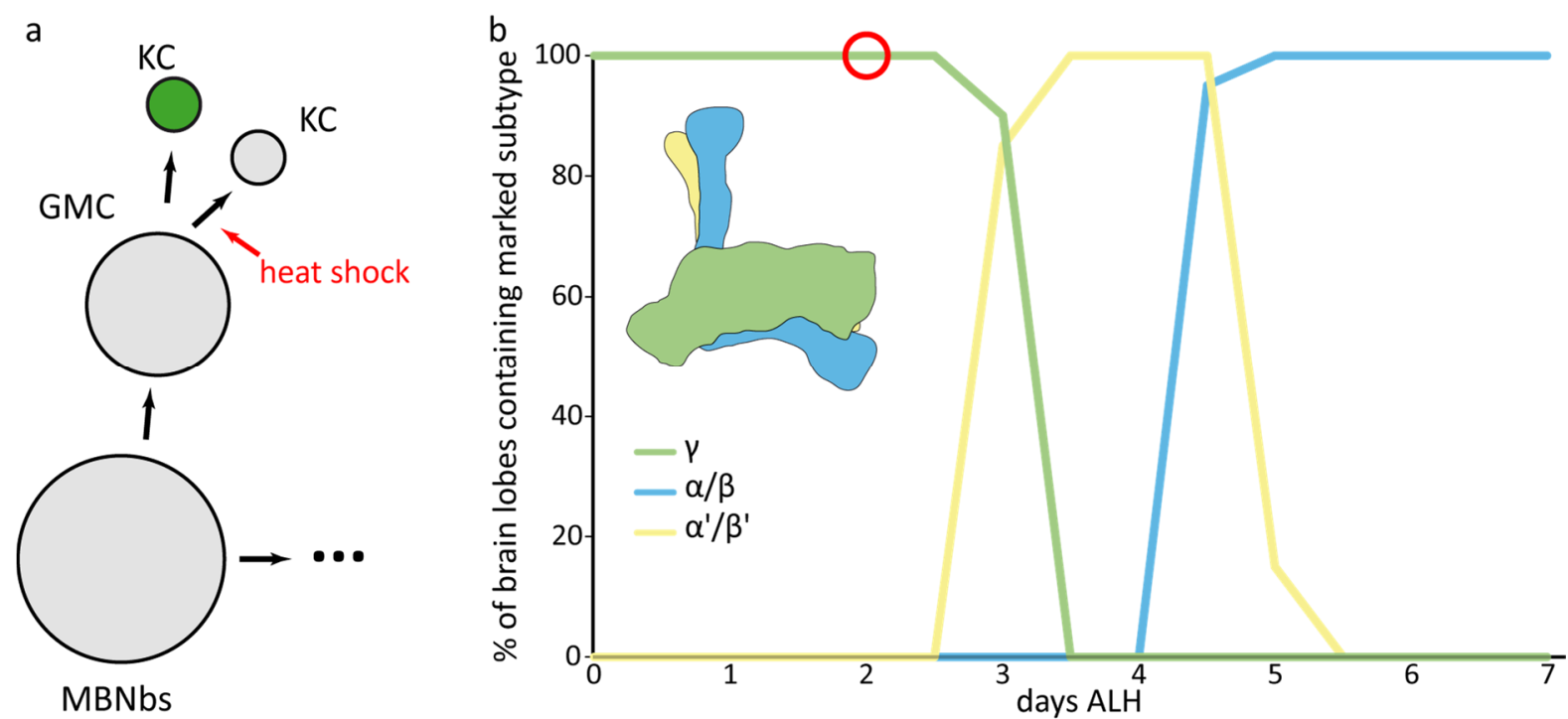

MBNbs

Figure 2.1: Generation of M ARCM single-cell clones. a M Bs develop from four mushroom body neuroblasts (MBNbs) that differentiate into ganglion mother cell (GMC) and ultimately in two KCs. In order to induce expression in a single $\mathrm{KC}$ the heat shock had to be given during GMC division. For further detail see 1.2.2, Fig. 1.2). $\mathbf{b}$ The three main $K C$ types develop sequentially during larval and pupal development ( $\gamma$-lobe green, $\alpha^{\prime} / \beta^{\prime}$-lobe - yellow, $\alpha / \beta$-lobe - blue; see also 1.4 .2 and Fig. 1.5). In order to restrict the transgene expression to $y$-KCs the heat shock was applied 2 days after larval hatching (ALH, red circle).

\subsubsection{In-vivo 2-Photon Calcium Imaging}

For calcium imaging experiments, female transgenic flies of the $F_{6}$ generation ( $3-7$ days old) were briefly anesthetized on ice and placed in a custom build shock-delivery chamber (Fig. 2.2) and fixed with a transparent tape. A hole was cut into the tape to expose the head and fix it by using UV-hardening dental glue. A drop of Ringer's solution (room temperature) was placed on 
top of the head. The cuticle of the head capsule was then opened with a fine stab knife to access the brain. To reveal the brain a very fine forceps was used to remove fat tissue and tracheae. The flies were then placed under the 2-photon microscope (20x water immersion objective) and checked for M ARCM -positive cell clones using a xenon lamp. In cases were the fixation of the fly and the head couldn't restrain the fly's movement they had to be excluded from the imaging experiment.

To monitor the fluorescence in the boutons of single KCs the laser was set to $940 \mathrm{~nm}$ to excite the calcium sensor GCaMP3 and the red fluorescent protein mCherry at the same time. A dichroic mirror was combined with a 500 to $550 \mathrm{~nm}$ and a 650 to $660 \mathrm{~nm}$ BP filter to record GCaM P3 and mCherry fluorescence simultaneously. Each imaging sequence was recorded at a frame rate of 4 $\mathrm{Hz}$ and 85 frames were taken (= $21.25 \mathrm{~s}$ ) at a resolution of $512 \times 512$ pixels. A custom build olfactometer was attached to the imaging chamber. A fine hypodermic-needle (diameter $=1.1$ $\mathrm{mm}$ ) was inserted into the imaging chamber to convey the odorized air to the fly's antennae at a flow rate of $1 \mathrm{ml} / \mathrm{s}$. The odor delivery was controlled with a custom-written LABVIEW program (Dr. Shubham Dipt) presenting subsequently the solvent M ineral oil (M Oil), 4-M ethylcyclohexanol (M CH - 1:750), 3-Octanol (3-Oct - 1:500) and 1-Octanol (1-Oct - 1:400). The odor onset was set to $6.25 \mathrm{~s}$ after recording onset and the odor stimulus lasted $2.5 \mathrm{~s}$. All imaging settings and recordings were controlled by the ZEN software of Zeiss. Depending on the complexity of the single KCS several planes in the lobe region of the MB were recorded to capture most parts of the axons. The imaging region was adjusted to capture either one or both hemispheres depending on the respective KCs being expressed on either one or both hemispheres, respectively. Each odor recording was separated by a $20 \mathrm{~s}$ break. If a fly had no responding cell all necessary planes were monitored and subsequently a z-stack was recorded. In a fly having at least one responding cell all necessary planes were monitored for their naïve odor responses and subsequently the training protocol was carried out (Fig. $2.2 \mathrm{f}-\mathrm{g}$ ). In the training $10 \mathrm{~s}$ after measurement onset the first odor (M CH or 3-Oct) was presented for $1 \mathrm{~min}$. With a time shift of $5 \mathrm{~s} 12$ electric shocks (90 V, $1.25 \mathrm{~s}$ shock, $3.85 \mathrm{~s}$ pause, total $60 \mathrm{~s})$ were paired with the first presented odor (CS+). After a resting period of $1 \mathrm{~min}$ the second odor (3-Oct or MCH, respectively) was presented for $1 \mathrm{~min}$ without the shocks (CS-). After the CS- offset the measurement went on for additional $30 \mathrm{~s}$. The control group received the same protocol but omitting the shock = no US control. To eliminate effects of odor identity in an associative training paradigm the animals were trained reciprocally (in one half of experiments $\mathrm{MCH}$ paired with the shocks, in the other half 3-0ct). After the training, flies were given a 3 min resting period to consolidate a short term memory. All previously measured planes were again monitored for their odor responses. Subsequently, a z-stack (resolution: 1024 x 1024 pixels) was recorded to capture the whole anatomy of the KC. Afterwards, the fly's brain was 
explanted and subjected to an immunohistochemical staining (see 2.2.4). Those flies that died during or after the training procedure were also subjected to an immunohistochemical staining but only analyzed for their pre-responses.
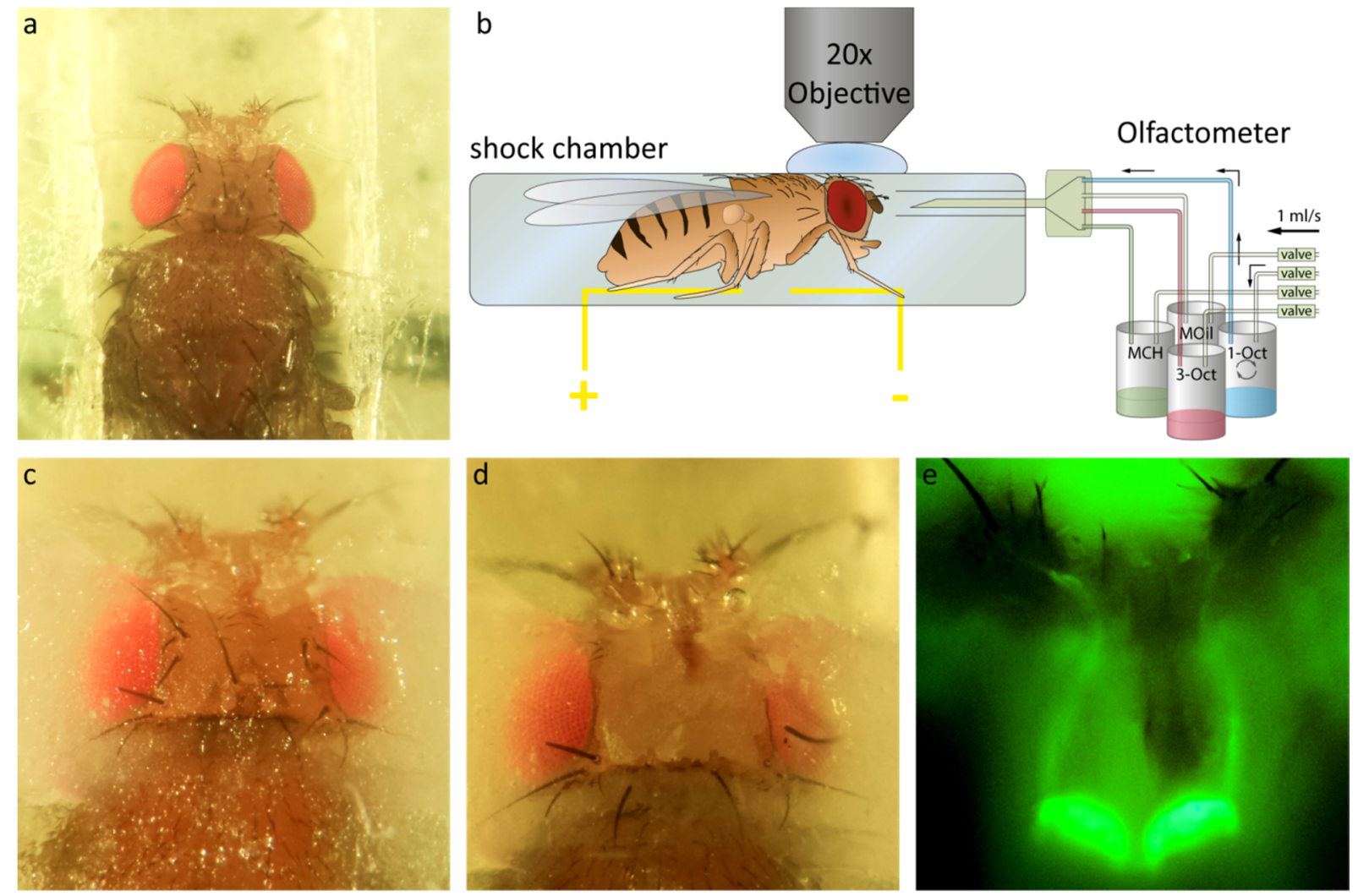

$\mathrm{f}$

associative training

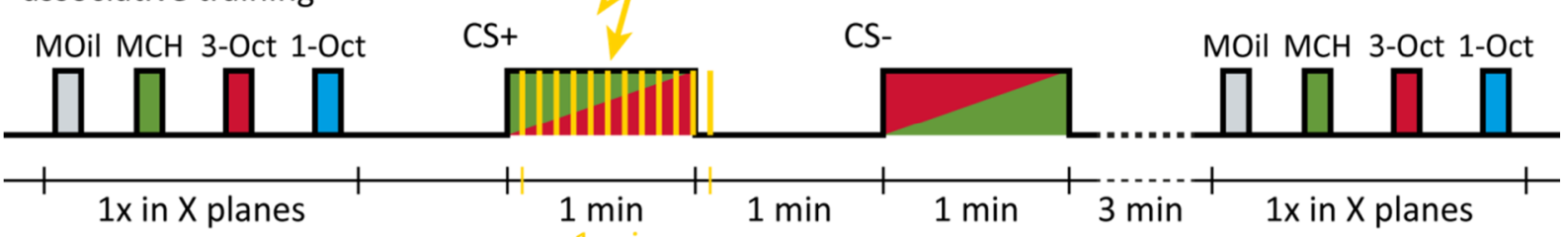

g

CS only control



Figure 2.2: 2-photon imaging setup with a custom build shock-delivery-chamber. a female flies are placed in the chamber and covered with transparent tape. $\mathbf{b}$ Schematic drawing of the 2-photon imaging setup with the attached olfactometer. c A hole of the size of the fly's head is cut into the tape still covering the antennae. UV-glue is positioned around the eyes and the neck to fix the head in the chamber. $\mathbf{d}$ A drop of Ringer's solution is put on top of the head and with a fine scalpel incisions are made along the eyes and at the back of the head. The cuticle piece was removed with a fine forceps and fat tissue and tracheae were removed to expose the brain. e Exemplary image of a brain expressing GFP in the antennal lobe excited with a xenon-lamp. $\mathbf{f}$-g schematic of the olfactory associative training protocol. In all necessary planes $(X)$ the solvent and the odors were presented. After odor presentations either $\mathrm{M} \mathrm{CH}$ (green) or 3-Oct (red) were presented for $1 \mathrm{~min}$ and paired with electric shocks (CS+, f). After a 1 min break the respective other odor was presented without a shock (CS-). For short term memory formation a 3 min resting period was given to 
the fly and subsequently the odors were presented in the same X planes. As a control the same procedure was applied to the control flies but omitting the shock pairing (g).

\subsubsection{Immunohistochemistry}

In order to investigate the anatomy and to assign the axonal branches of the single $\mathrm{y}$-KCs to the corresponding $\gamma$-lobe compartment, flies were removed from the imaging chamber and anesthetized on ice. Subsequently, the flies were pinned to a Sylgard-dish and their brains were dissected in ice cold Ringer's solution. Afterwards, the brains were fixed in $4 \%$ PFA for $2 \mathrm{~h}$ at $4^{\circ} \mathrm{C}$. To remove PFA residues, brains were rinsed three times for 20 min each in phosphate buffered saline (PBS) and subsequently incubated in blocking solution (see 2.1.5) for $2 \mathrm{~h}$ at room temperature. The brains were then incubated with the primary antibodies ( $\alpha$ GFP and $\alpha \mathrm{DLG}$ ) that were diluted in blocking solution overnight at $4^{\circ} \mathrm{C}$. Subsequently, the brains were rinsed three times for 20 min each in PBST (see 2.1.5) at room temperature. After rinsing, the secondary antibodies (Alexa Fluor 488 and Alexa Fluor 633) were diluted in blocking solution as well and the brains were incubated over night at $4^{\circ} \mathrm{C}$. The brains were rinsed again in PBST for 20 min and afterwards two times in PBS for 20 min each at room temperature. Two transparent tape rings were stuck onto microscope slides to form a chamber for the brain. After the last washing step, the brains were placed into these chambers pointing the anterior site upwards and embedded in Vectashield mounting medium. The chamber was covered with a cover slip and sealed with nail polish. The embedded brains were then imaged under a confocal microscope.

\subsubsection{Confocal Microscopy}

Confocal microscopy was performed using a SP8 laser scanning microscope of Leica. The brains were positioned with the help of a 10x air objective. A 20x glycerol/water objective was used for detailed image acquisition. Microscope settings were adjusted in the Leica software LSAX. To image the GFP-A488 fluorescence the Argon-laser was set to $488 \mathrm{~nm}$. The DPSS-laser (561 nm) was used to excite the intrinsic expressed mCherry-protein and the HeNe-laser $(633 \mathrm{~nm})$ for excitation of DLG-A633. The laser power was adjusted for each probe accordingly to get the optimal intensity without bleaching the probe. The zoom was set to 2 and the resolution to $1024 \mathrm{x}$ 1024 pixels resulting in a pixel dwell time of $600 \mathrm{~ns}$ with a pixel size of $0.283 \mu \mathrm{m}$. For a better reduction of noise 2 frames per z-position were recorded and averaged. Pinhole size was kept constant at $1 \mathrm{AU}$. For fluorescence detection the implemented two hybrid detectors were used. 
As the brains contain three different fluorophores the image acquisition was done in a sequential manner. The z-step size was kept constant at $1.04 \mu \mathrm{m}$.

\subsubsection{Image Analysis}

All image analysis was performed using Imagej. A custom-written plugin that is based on the StackReg-plugin of ImageJ ("Color Batch Processing5", Dr. Ulrike Pech \& Dr. Shubham Dipt) was used to align the recorded stacks of the 2-photon microscopy. Thereby, the plugin used the red channel (mCherry recording) to align the frames to a reference frame of that sequence and applied the alignment steps to the green channel (GCaMP3 recording). Each aligned stack was monitored for alignment errors and if necessary re-aligned using the ImageJ-plugin "Template Matching" published by Qingzong Tseng. In this plugin the "Align slices by cvMatchTemplate" mode was used (matching method: normalized correlation coefficient, search area $=0$, bilinear interpolation for subpixel translation) to re-align the green channel. With a custom-written macro ("Realignment by Result Table", Dr. Carlotta Martelli) the x-y correction was applied to the red channel.

With the confocal imaging stacks each $\mathrm{Y}$-KC was reconstructed by marking all parts belonging to that $\mathrm{y}-\mathrm{KC}$ and cutting it out from the background. In case of having more than one $\mathrm{y}-\mathrm{KC}$ in one hemisphere the $\gamma$-KCs had to be disentangled and were separately reconstructed by marking the cell parts belonging to only the respective $\mathrm{Y}-\mathrm{KC}$. With the 3D projection function of Image the cells were three dimensionally visualized by interpolating the steps in z-direction. Thereby, they could be rotated over one axis in single degree steps, to better identify the $\mathrm{Y}$-KC axon branches and compare them to the in-vivo imaging.

Each identified bouton was marked with a circular region of interest (ROI) containing always 12 pixels. If applicable (post training analyzable) the same bouton was marked in the imaging sequence after the training for later comparison. With the help of the 3D-reconstrucion and the in-vivo z-stack the boutons were assigned to the $y$-lobe compartment they belong to. The mean fluorescence intensities of all identified boutons were measured for each imaging frame in the GCaMP3 recordings as well as in the mCherry recordings. In Excel 2010 the $\Delta F / F_{0}$ values were calculated by subtracting the background $\left(F_{0}\right)$ for each bouton (average of time frame $3-22$ before the stimulus) $\Delta \mathrm{F}=\mathrm{F}-\mathrm{F}_{0}$ and subsequently divided by the background. To further reduce movement artefacts in z-direction the $\Delta F / F_{0}$ values of mCherry were subtracted from $\Delta F / F_{0}$ values of GCaMP3 and afterwards a sliding average (3 frames) was applied to smooth the calcium transients. The boutons of each imaging plane were sorted by $\mathrm{y}$-lobe compartment. The response 
of a bouton was calculated by averaging 5 frames around the peak value. To prevent false positive peaks that can occur due to movement artefacts or spontaneous activity that is stimulus unrelated, only those peaks were taken into account that occurred around the stimulus phase (frame $25-44,=5 \mathrm{~s}$ ).

\subsubsection{Bouton Similarity Analysis - Activity Corrected Correlation}

In order to analyze the synchrony of each $\mathrm{y}$-lobe compartment, a new similarity measurement was derived in collaboration with Dr. Bart Geurten, called Amplitude Corrected Correlation (ACC) index. For each bouton the cross-correlation coefficient (normalized to the autocorrelation, see Equ. 1) to each other bouton of the same cell was calculated, resulting in a number between 0 and 1 representing the fraction of the autocorrelation. Subsequently, the median of all crosscorrelation coefficients for all boutons in each possible combination of y-lobe compartments was calculated. The median of the internal and external cross-correlation coefficient of all boutons assigned to this $y$-lobe compartment was calculated as well. As the boutons within each y-lobe compartment can respond differently, they do not necessarily have a $100 \%$ correlation within the compartment. At the end of this stage the internal and external $y$-lobe compartment similarity is represented by a number between zero and one.

The following factors focus the ACC on the absolute response amplitude and the contrast between response amplitudes. This was necessary because respiration or swallow movements might produce precisely synchronous noise with low amplitudes in all boutons, especially for those odors, that the $\mathrm{y}$-KCS did not respond to. Therefore the median amplitude of both $\mathrm{y}$-lobe compartments normalized to the maximum of the cell (see Equ. 2-3) were factored in. The high correlation coefficients resulting from synchronous noise are decreased by their overall low amplitude of response. Furthermore, the ACC index for $y$-lobe compartment comparisons is adjusted by a contrast measure of the respective compared median amplitudes (see Equ. 4). The results from a Michelson contrast computation of both median amplitudes were subtracted from 1 , so that identical amplitudes equaled a factor of 1 . The cross-correlation coefficient and the two respective correction factors resulted in a product (the ACC index, see Equ. 5), which is numerically bound between 0 and 1 , where 1 represents identity of both responses. Importantly, this does not have to necessarily be the case for internal ACCs. Internal ACCs would only reach 1 if all boutons of the given $y$-lobe compartment show the exact same response, as the correlation coefficient is the median of all cross correlations inside the lobe. Hence, this kind of analysis presents a robust quantification of similarity between calcium dynamics that combines the temporal domain of the response as well as its amplitude. 
Equation 1: normalized cross-correlation

$C I_{1} \star C I_{2}=\frac{\int C I_{1}^{*}(\tau) C I_{2}(t+\tau) \delta \tau}{\sqrt{\sum_{\alpha \epsilon C I_{1}} \alpha^{2}} \cdot \sqrt{\sum_{\beta \epsilon A C I_{2}} \beta^{2}}}$

$\mathrm{Cl}_{1} \star \mathrm{Cl}_{2}$ is the normalized cross-correlation where $\mathrm{Cl}_{1}$ and $\mathrm{Cl}_{2}$ are two bouton calcium response time series, with $\star$ denoting the cross-correlation between two time series. $\mathrm{t}$ is the time point of the response and $\tau$ is the phase lag of the correlation. $A_{1}$ and $A_{2}$ are the respective maximum amplitudes of $\mathrm{Cl}_{1}$ and $\mathrm{Cl}_{2}$. $\alpha$ and $\beta$ denote each sample of the respective response, and $\mathrm{n}$ is the entirety of all bouton response amplitudes of one cell. The quotient returns a number in the range 0 - 1 .

Equation 2: Amplitude correction for intra lobe comparisons

$A_{a}=\frac{A_{n}}{\max \left(A_{1 \rightarrow n}\right)}$

Equation 3: Amplitude correction for inter lobe comparisons:

$A_{a}=\frac{\frac{A_{1}+A_{2}}{2}}{\max \left(A_{1 \rightarrow n}\right)}$

Equation 4: Amplitude contrast for inter lobe comparisons:

$A_{c}=1-\left(\frac{A_{1}-A_{2}}{A_{1}+A_{2}}\right)$

Equation 5: Amplitude Corrected Correlation:

$A C C=C I_{1} \star C I_{2} \times A_{a} \times A_{c}$

\section{ACC index contrast}

To test the hypothesis that the $y$-lobe compartments are individual functional units, another Michelson contrast was calculated. This time the internal ACC was subtracted from the median external ACCs of this $\gamma$-lobe compartment and divided by the sum of both (Equ. 6). The resulting value should be 1 if the $\psi$-lobe compartment is highly correlated to itself but not to all the other $\psi$ lobe compartments. Negative values arise if all boutons in the $\psi$-lobe compartment are correlated stronger to boutons of other $\gamma$-lobe compartments than to themselves. This contrast value was calculated for all $\mathrm{y}$-KCS and its median and the corresponding $95 \%$ confidence interval $(\mathrm{Cl})$ derived. If this $\mathrm{Cl}$ does not cross the zero value, the $\mathrm{y}$-lobe compartment is significantly more correlated to itself (positive median contrast) than to the other $\gamma$-lobe compartments. If this is the 
case but the median contrast is negative the $y$-lobe compartment is not correlated to itself but more to other $\mathrm{y}$-lobe compartments and ceases to be a functionally independent unit. If the $95 \%$ $\mathrm{Cl}$ crosses the zero value, there is no statistical evidence that this $\gamma$-lobe compartment is a functional independent unit.

Equation 6: ACC index contrast:

$A C C_{C}=\frac{A C C_{\text {intra }}-\overline{A C C_{\text {lnter }}}}{A C C_{\text {intra }}+\overline{A C C_{\text {lnter }}}}$

\section{ACC difference}

In order to investigate the effect of associative learning on the synchrony of the different $y$-lobe compartments the ACC was employed again. The influence of learning could be uncovered by simply subtracting the combined pre-training ACC for each $y$-lobe compartment from the ACC after a training trial. Such a difference matrix was calculated for each cell and individually for CS+, CS- and control conditions. The medians and the $95 \% \mathrm{Cl}$ of the median between all cells of a given condition were calculated. The $95 \% \mathrm{Cl}$ was used again to test for significance. If the $\mathrm{Cl}$ did not cross the zero value the $y$-lobe compartment had significantly changed its synchrony.

\subsubsection{Cluster Analysis}

To test whether synchrony or asynchrony of boutons arises from boutons grouping together an agglomerative hierarchical cluster analysis (AHC) was performed in collaboration with Dr. Bart Geurten, using the peak response amplitude ( $3 x$ higher than the standard deviation) and its latency as the feature space. The AHC was based on the Euclidean distance between feature sets and the Ward's criterion (reviewed: Murtagh and Contreras, 2012, 2017). In order to compare both features the data was scaled by dividing each data point by the respective data standard deviation (z-score). Each bouton response was treated in the beginning as a single cluster and then merged in order of their minimal distance to the next cluster (Ward's minimum variance method, Ward, 1963). When the cost to merge two clusters rose rapidly, distinct clusters were merged and the native segregation of the data was reached. This point was determined in all cases to be 4 clusters. These clusters were called bouton response classes (BRCs) and the respective means of all responses assigned to those clusters were their centroids. The cluster borders were determined by creating the Voronoi cells around the centroids of each cluster. These borders were used to categorize how the bouton responses after the training (CS+, CS- and control) fall into the built clusters. The amounts of boutons of the $y$-lobe compartments that fell 
into a BRC were calculated and plotted as color coded matrix fields for the pre- and post-training conditions. The median fractions of boutons across $\gamma$-lobe compartments falling into those clusters were plotted as bar graphs (error bars indicate the $95 \% \mathrm{Cl}$ ). For all plots the CS+ condition is colored in red, CS- in green and control in blue. To investigate the internal variance of the clusters after the training an internal Euclidean distances matrix between all boutons of a BRC was calculated for each $\gamma$-KC. In each $\gamma$-KC the mean Euclidean distance of each BRC (1-4) was calculated and subsequently summed up. These sums for each $\mathrm{\gamma}-\mathrm{KC}$ were plotted as box plots (upper and lower quartiles) for the three post-training conditions. Grey lines indicate median, whiskers $1.5 \mathrm{x}$ interquartile distances. In order to determine the dispersion of the BRCs in the $\mathrm{\gamma}$ lobe compartments Shannon's information entropy was calculated using the MatLab Implementation of Will Dwinnell. It measured how many bits are needed to encode the combination of BRCS in each $y$-lobe compartment. The median information entropy for each training condition of each $\gamma$-lobe compartment was plotted as color coded matrix. The median information entropy across $\psi$-lobe compartments was quantified and plotted as boxplots (same as for BRC fractions).

\subsubsection{Statistics}

Boxplots indicate the lower and upper quartiles color coded by $\gamma$-lobe compartment ( $\gamma 2$ - green, p3 - yellow, p4 - purple, p - blue). Notches indicate median and $95 \% \mathrm{Cl}$. Whiskers indicate $1.5 \mathrm{x}$ STD. Black squares indicate the means. Statistics were made using OriginPro 8.5G and GraphPad Prism 7.04. Tests for normal distribution were done using the Shapiro-Wilk test. If at least one group showed no normal distribution the Mann-Whitney-U test was applied; for more than two independent groups, the Kruskal-Wallis ANOVA with the post hoc Dunn's multiple comparison test was applied. If more than two dependent non-normally distributed groups were compared the Friedman ANOVA with the post hoc Dunn's multiple comparison test.

ACC and cluster analysis were established, plotted and statistically analyzed in MatLab in collaboration with Dr. Bart Geurten. To test the significance of the difference before to after the training in the cross-correlation analysis and the ACC indices the median and its $95 \% \mathrm{Cl}$ of the post-conditions were calculated. The median of the pre-condition was subtracted from each postcondition. If the $95 \% \mathrm{Cl}$ of the difference was not crossing the 0 -value a $5 \%$ significance could be assumed (asterisk in the matrix fields, see Fig. 3.14). The fractions of BRCs in the three training conditions (CS+, CS- and control) were compared using a multi nominal $x^{2}$-test (Fig. 3.16 b). Fisher's permutation test was used to compare the inner BRC variance (Fig. $3.16 \mathrm{c}$ ) and the Shannon's information entropy of the $\gamma$-lobe compartments in the three training conditions (Fig. 52 
Materials and M ethods

3.17 b). All multiple comparisons were corrected with the Benjamini-Hochberg false discovery rate (FDR) procedure. 


\section{Results}

\subsection{Establishing Single Kenyon Cell Calcium Imaging}

\subsubsection{Generation of Single $\mathrm{y}$-KCs with the MARCM -Technique}

In order to investigate how single KCs code for odors and how aversive olfactory associative conditioning alters synaptic plasticity the Mosaic Analysis with a Repressible Cell Marker (MARCM, Lee and Luo, 1999) was employed (see 1.2.2 and Fig. 1.2). In brief, this genetic system induces the expression of transgenes on the single cell level based on the FLP/FRT system. Each cell contains the genes of interest and the GAL4 repressor Gal80 heterozygously repressing the expression of those genes. Furthermore, the cells are carrying a heat shock inducible flippase (hsFLP) and the FRT19A on the first chromosome. The FLP/FRT recombination is heat shock inducible through the heat shock protein hsp70 (Ashburner and Bonner, 1979). If the heat shock is given during a division of the ganglion mother cell (Fig. 1.2) the hs-FLP recombines at the FRT sites thereby producing one daughter cell homozygous for the genes of interest and the other daughter cell homozygous for the repressor which leads to expression in only a single cell clone (Fig. 3.1. d). If the heat shock occurs during the division of a neuroblast more than one cell clone is labeled (Fig. 3.1 b-c). In this study, the 5HT1B-GAL4 (Yuan et al., 2005) was used to restrict the expression to the $\mathrm{y}$-KCs (Fig. 3.1. a). To trace the KCs, a hexameric mCherry inserted behind 20 copies of UAS (Shearin et al., 2014) was used. To monitor activity-dependent calcium changes in the $y$-KCs GCaM P3 inserted behind 20 copies of UAS (Tian et al., 2009) was used. 

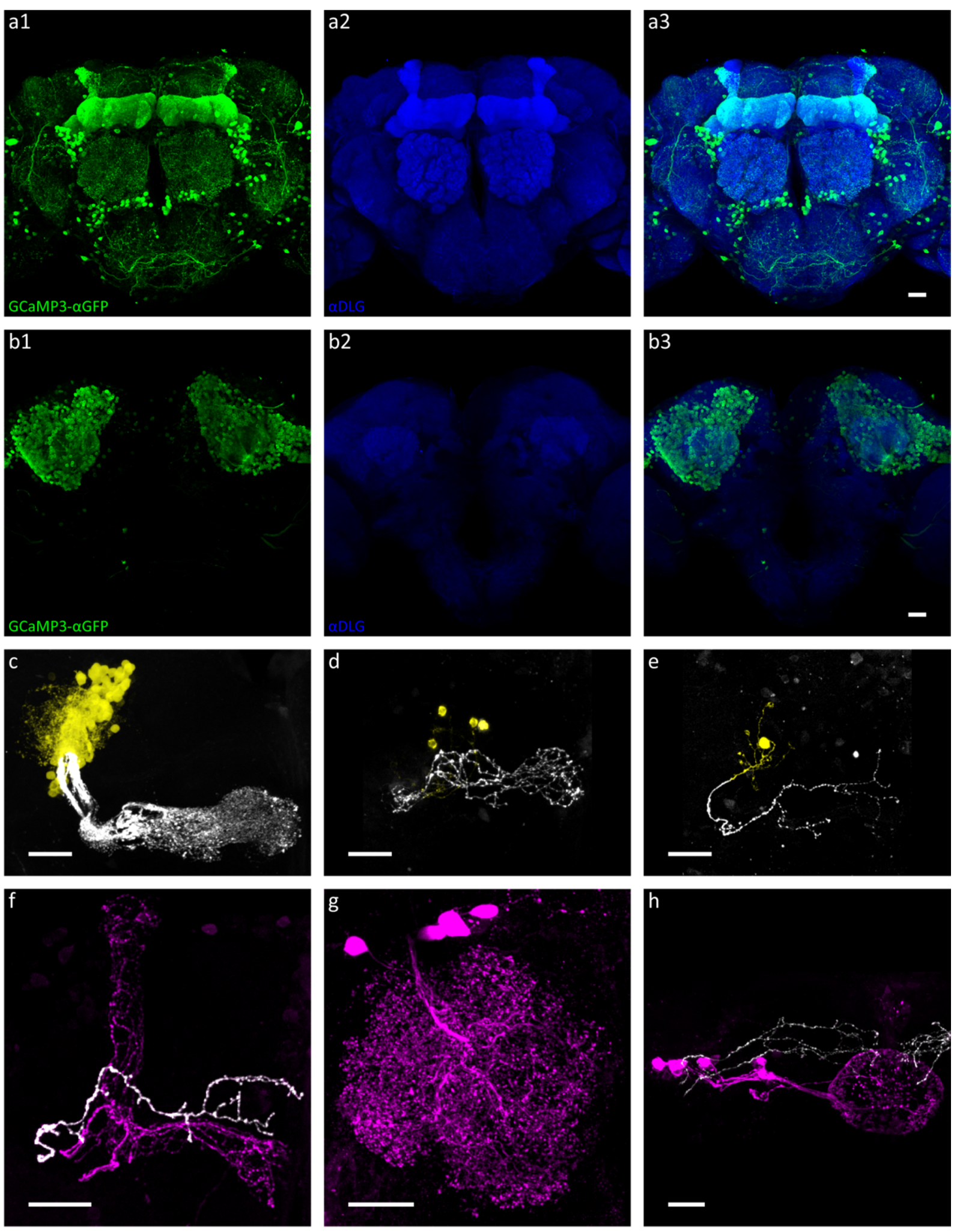

Figure 3.1: Generation of single $y$-KC clones with MARCM driven by 5-HT1B-GAL4. All images show the maximum projection of a confocal z-stack. a-b Whole mount brain preparation of a fly expressing UASGCaMP3 under 5-HT1B-GAL4 control showing in the MB predominantly expression in the $y$-lobe and additional innervations outside the MB. Immunohistochemistry was applied to enhance fluorescence intensity of GCaMP3 ( $\alpha$-GFP-Alexa488, green, $\mathbf{a l}$ and $\mathbf{b} \mathbf{1}$ ) and stain the post-synaptic protein DLG ( $\alpha$-DLGAlexa633, blue, a2 and b2) to visualize the neuropils of the brain. The merge of both channels is shown in a3 and b3. The anterior part of the brain including the MB-lobes is shown in $\mathbf{a}$. The posterior part of the brain including the calyx and cell body region of the MB is shown in $\mathbf{b}$. c-e Labelling of $\mathrm{Y}-\mathrm{KC}$ clones with the $M A R C M$ technique. In white the anterior part of the MB including the lobe region and peduncle is shown, 
whereas yellow shows the posterior part of the MB including the calyx and cell body region. Depending on the timing of heat shock during larval development different numbers of $\mathrm{y}-\mathrm{KC}$ clones are produced. These numbers can range from multiple $(\approx 40, \mathbf{c})$, few $(4, \mathbf{d})$ or to ultimately a single clone $(\mathbf{e})$. $\mathbf{f}$ - $\mathbf{h}$ intrinsic mCherry expression of $\gamma$-KCs (white) and $\gamma$-lobe extrinsic neurons (magenta) $\mathbf{f}$ In rare cases the MARCM technique additionally labeled $\alpha / \beta-K C$ s that are partially part of the $5-H T 1 B-G a l$ driver. In this example a single $\gamma-K C$ clone (white) plus $6 \alpha / \beta-K C$ clones (magenta) were labeled. $\mathbf{g}$ in one sample a $\gamma$-lobe extrinsic cell cluster innervating the AL was additionally expressing mCherry (magenta). $\mathbf{h}$ in another sample mCherry was additionally expressed in cells innervating the ellipsoid-body (magenta) besides having labeled $2 \gamma$-KC clones in the left hemisphere and $2 \gamma$-KC clones in the right hemisphere (white). Scale bars $=20 \mu \mathrm{m}$.

The GAL4 driver line 5HT1B leads predominantly to transgene expression in $\mathrm{Y}^{\mathrm{K} C \mathrm{~K}}$ (Fig. $3.1 \mathrm{a}$ ). However, it additionally expresses transgenes in some $\alpha / \beta-K C$, some $\alpha^{\prime} / \beta^{\prime}-K C s$, as well as neurons outside the MB (e.g., neurons innervating the antennal lobes or ellipsoid body, Fig. $3.1 \mathrm{f}$ h). In most cases the M ARCM technique resulted in $\mathrm{Y}$-KC specific transgene expression (Fig. $3.1 \mathrm{c}-$ h) plus in rare cases $\alpha / \beta$-KCs (Fig. $3.1 \mathrm{f}$ ) and/or neurons outside the MB (Fig. $3.1 \mathrm{~g}$-h). The overall efficiency of M ARCM-mediated $\gamma$-KC expression was $\approx 64.4 \%$ ( 275 out of 427 prepared flies). For the data set of $y$-KCs expressing cytosolic GCaM P3 the efficiency was $\approx 62.9 \%$ (151 out of 240 ). Of these 151 animals $120(\approx 79.5 \%$ ) had transgene expression in a sufficiently low number of KCs so that they could be differentiated ( 1 - 3 KCs per hemisphere) and were therefore used for calcium imaging experiments. In rare cases some $\mathrm{y}$-KCs had only mCherry expression without detectable GCaMP3-fluorescence and were therefore not used for calcium imaging experiments. The $\mathrm{Y}$-KCS showed highly variable axonal anatomies where the axons extended side branches in different parts of the lobe (Fig. 3.2) ranging from more simple axons (less branches, e.g., Fig. 3.2 KC7) to more complex axons (many branches, e.g., Fig. $3.2 \mathrm{KC19}$ ). Nonetheless, in all cases $\mathrm{y}$-KCs projected their axons along the entire length of the $\mathrm{y}$-lobe. 

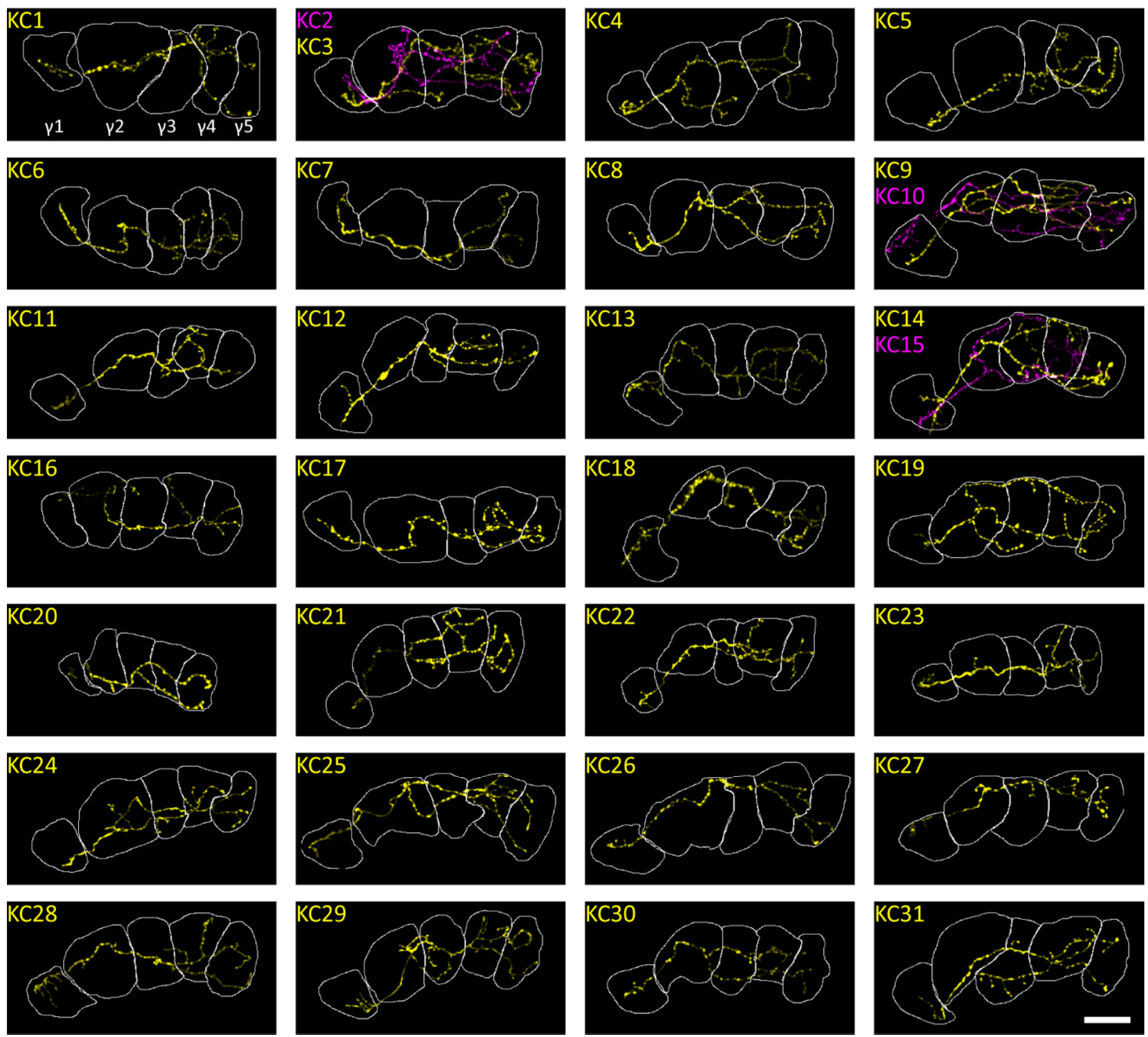

Figure 3.2: Z-stack reconstruction of single $\gamma$-KCs indicating their diverse anatomies. Each panel shows the maximum projection of the reconstructed $\mathrm{y}$-KCS (yellow), which responded reliably and were analyzed in this study. In three cases $2 \gamma$-KCs were expressed in the same MB on the same hemisphere (magenta $\gamma$-KCs in addition). The white outlines indicate the borders of $\gamma$-lobe borders ( $\gamma 1-\gamma 5)$. Scale bar $=20 \mu \mathrm{m}$.

\subsubsection{Boutons in $\gamma$-KC Axons are Rich in the Pre-Synaptic Protein Bruchpilot}

The axons of $y-K C s$ provide the pre-synaptic output of the MB to the M BONs that in turn guide the behavior towards approach or avoidance (Aso et al., 2014b). Calcium imaging (Fig. 3.4) as well as confocal z-stacks (Fig. $3.1 \mathrm{c}-\mathrm{f}, \mathrm{h}$ ) revealed that single $\mathrm{y}$-KCs exhibited spherical structures along their axons. These structures will be called boutons from here and after. To test whether these boutons are putative synaptic connections to downstream neurons (such as MBONs) the BRP-GFP (Fouquet et al., 2009) construct was expressed in single $\mathrm{Y}^{\mathrm{K} C \mathrm{C}}$ (Fig. 3.3).

The expression of BRP-GFP in single $\mathrm{\gamma}$-KCs has shown that the axonal boutons were enriched in BRP (Fig. 3.3). Therefore, it was of great interest to investigate single boutons as they were likely the synaptic site of the microcircuit between KCs, DANs, and MBONs, which change their synaptic 
activity in the course of olfactory associative learning (Cognigni et al., 2018; Davis, 2011; Kaun and Rothenfluh, 2017). Hence, single boutons in each $\mathrm{y}-\mathrm{KC}$ were analyzed for their odor responses and training-induced changes in the calcium dynamics (next sections).
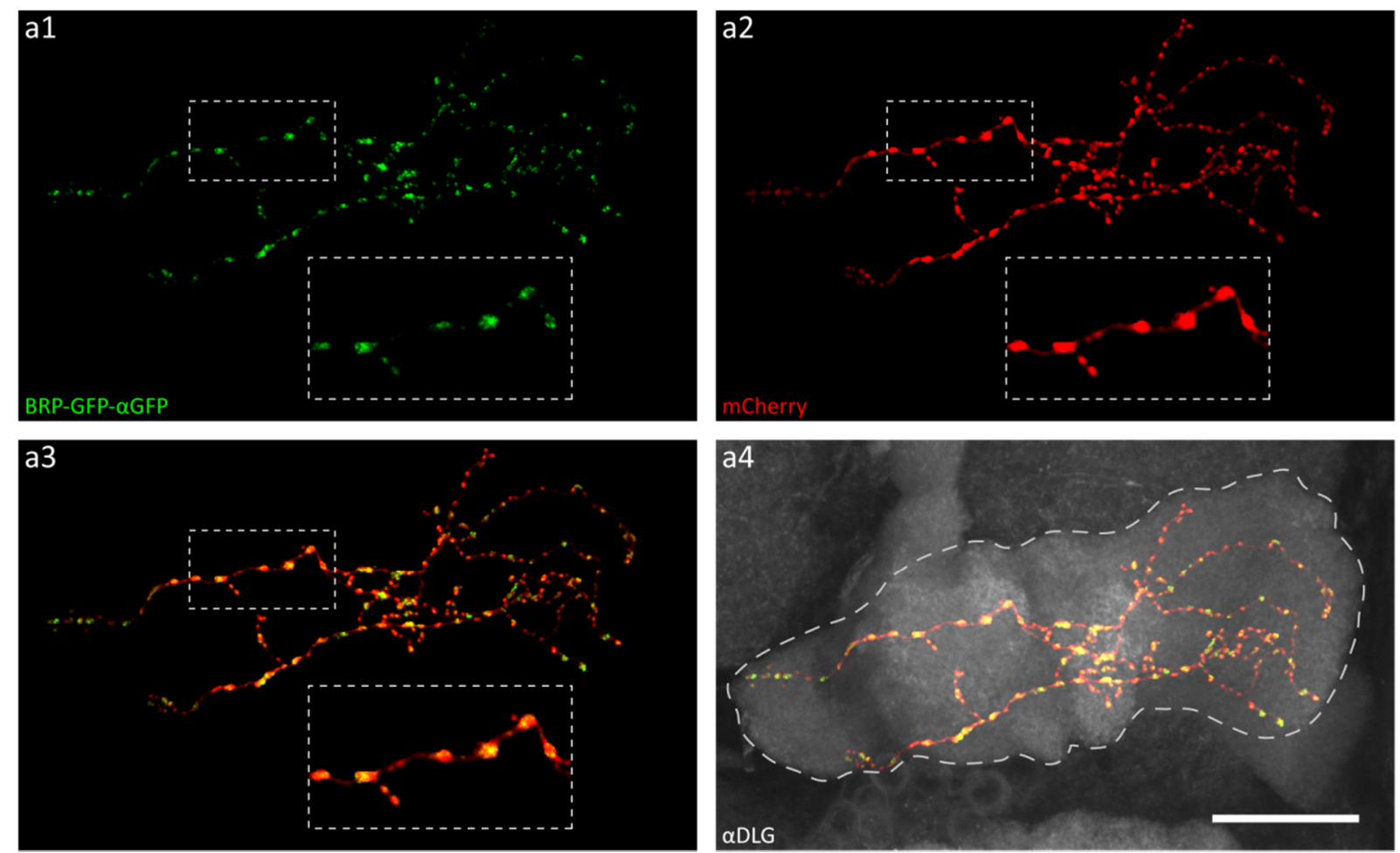

Figure 3.3: M aximum projection of immunohistochemical staining shows that $\gamma-\mathrm{KC}$ boutons are enriched in BRP. a1 BRP-GFP expressed in two single $\gamma$-KCs stained against GFP (green) showing only labeling in spheric structures. a2 Intrinsic mCherry expression (red) of the same two $\gamma$-KCs showing the axonal branches with its boutons. a3 Merge of al and a2 showing that BRP-GFP is mainly present in the boutons (yellow color mixture) and not in between. Insets of a1-a3 (dashed rectangles) show a 1.8x magnification of an axonal branch. a4 Overlay of a3 and the staining against the post-synaptic protein DLG (grey) indicating the M B and the $\gamma$-lobe (dashed outline). Scale bar $=20 \mu \mathrm{m}$.

\subsubsection{Calcium Imaging of Single $\mathrm{p}-\mathrm{KCs}$}

The calcium reporter GCaM P3 (Tian et al., 2009) is a convenient tool to monitor neuronal activity as it shows fluorescence changes upon calcium influx into a neuron (Katz and Miledi, 1965; Littleton et al., 1994; Nakai et al., 2001). The above mentioned MARCM technique (see 1.2.2, 2.2.2 and 3.1.1) allows monitoring of the activity of single neurons such as KCs. The $y$-KCs were shown to be involved in short term memory (Blum et al., 2009; Qin et al., 2012; Zars et al., 2000). To investigate how these KCs convey the odor information and how the activity is changed in the course of associative olfactory memory the serotonin receptor driver 5-HT1B-GAL4 was used to predominantly restrict the expression of GCaMP3 and mCherry to the $y$-lobe KCs. 
An imaging plane contained several branches of either one or several $\mathrm{\gamma}$-KC-axons in which boutons were detectable (Fig. 3.3, Fig. 3.4 a-b, top). Plotting the time course of the calcium dynamics of such a bouton revealed if it was responding to none, one, or several odors (Fig. 3.4 ab bottom). Only the boutons that could be identified before and after the training were taken into the analysis. In the case of $\mathrm{p}$-KCs only responding before the training, all identifiable boutons were taken into the analysis. This resulted in on average $\approx 62.74$ boutons ( \pm 12.6 STD) per $\gamma$-KC axon. Thereby, the base line fluorescence as well as the maximum amplitude of an axon differed between cells. As the MB $\mathrm{\gamma}$-lobe is anatomically compartmentalized by the extrinsic innervation of DANs and MBONs and functionally distinct in these regions (Aso et al., 2014a), it is of great importance to assign the monitored boutons to these compartments. This was achieved with the help of the anti-DLG staining and the three dimensional reconstruction of each $\gamma-\mathrm{KC}$ (see methods 2.2.4-2.2.6; Fig. $3.4 \mathrm{c}-\mathrm{d}$ ). The borders of $\mathrm{y}$-lobe compartments could be defined because less innervation of extrinsic neurons exists between the compartments. Due to the dorsal imaging angle it was not possible to monitor the branches and corresponding boutons inside the $\gamma 1$ compartment in most flies. Therefore, the $\gamma 1$ compartment was left out in this study. All other identified boutons were assigned to and sorted by the corresponding $\gamma$-compartment (Fig. 3.5). 

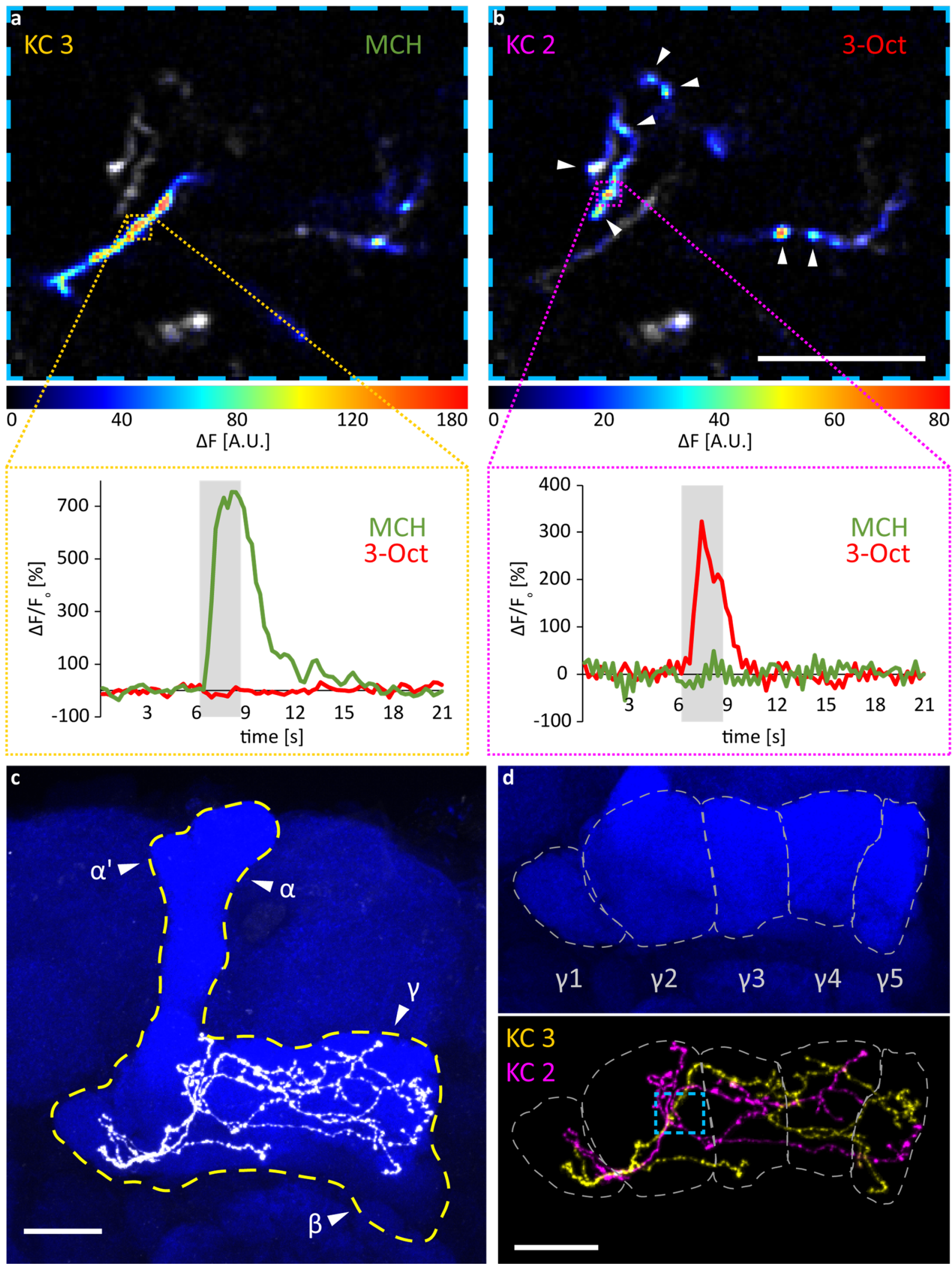

Figure 3.4: Calcium imaging of axonal boutons in single $\mathrm{\gamma}$-KCs. a exemplary imaging plane of a fly expressing GCaMP3 and mCherry in two cells in one hemisphere. False-color coded odor evoked calcium activity to $\mathrm{MCH}$-stimulation is superimposed on the mCherry background fluorescence (top, gray). Blue dashed outline refers to the imaging plane location shown in $\mathbf{d}$. In the bottom row the calcium dynamics of a single bouton measurement from one axonal branch is depicted showing an increase in fluorescence only for $\mathrm{MCH}$ stimulation (grey shaded area shows odor stimulus window). b Same imaging plane as in a showing the odor-evoked activity to 3-Oct stimulation superimposed on the mCherry background with white arrow 
heads indicate exemplary boutons (top). In the bottom row the calcium dynamics of a single bouton measurement from second $\mathrm{y}-\mathrm{KC}$ axonal branch is depicted showing an increase in fluorescence only for 3Oct stimulation (grey shaded area shows odor stimulus window). c immunohistochemical z-projection of the same fly brain after the calcium imaging procedure showing the antibody staining against the postsynaptic protein DLG (blue) and the intrinsic fluorescence of mCherry in $3 \gamma$-KCs (white). Yellow dashed outline indicates M B-lobe region. White arrow heads indicate the respective MB-lobes $\left(\alpha, \beta, \gamma\right.$, and $\left.\alpha^{\prime}\right)$. d The top row shows the same DLG-staining (blue, top) used to define the five $\gamma$-lobe compartments (dashed outlines). The bottom row shows the reconstruction of two $\mathrm{\gamma}$-KCs (colors correspond to a). The third cell was expressing only mCherry and therefore excluded from the analysis and for reasons of clarity and comprehensibility not shown. The compartment outlines could be used to assign the $\mathrm{\gamma}$-KC branches and their respective boutons to the corresponding $\gamma$-lobe compartments. Blue dashed rectangle indicates the imaging plane shown in $\mathbf{a}$ and $\mathbf{b}$. Scale bars $=20 \mu \mathrm{m}$.

a

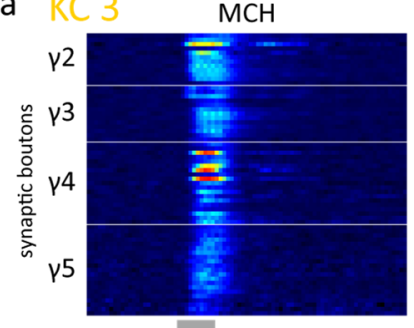

b $\mathrm{KC} 2$

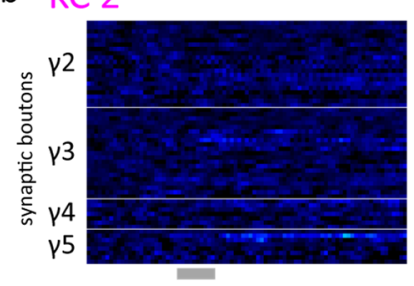

3-Oct
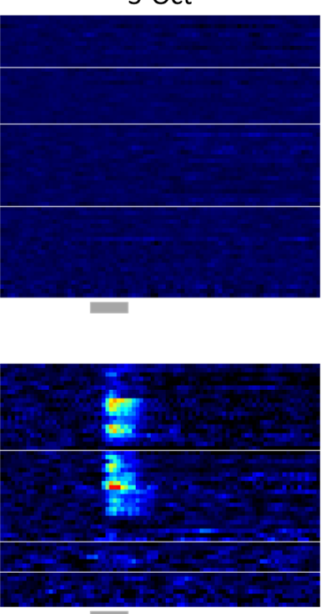

1-Oct
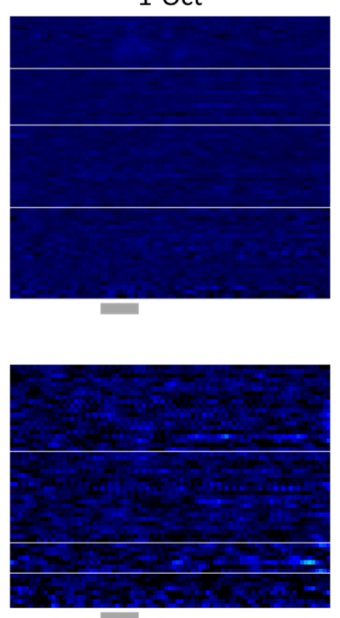

MOil
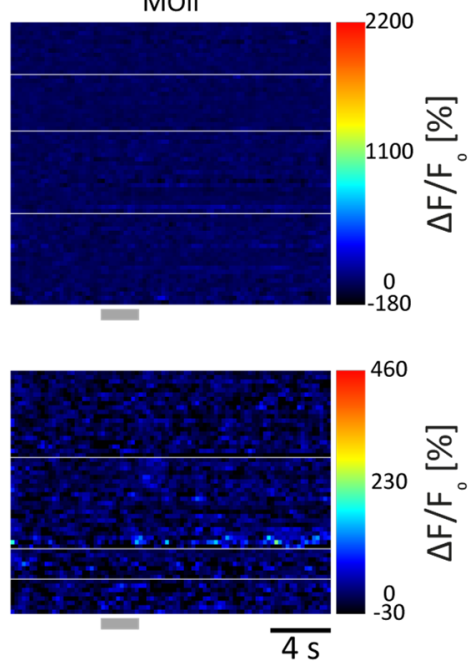

Figure 3.5: False color coded heat maps showing single bouton responses in single $\gamma$-KCs. a Response profile of the yellow $\gamma$-KC from Fig. 3.4. Each row shows the $\Delta F / F_{0}[\%]$ over time of a single bouton sorted by its $\gamma$ lobe compartment $(\gamma 2-\gamma 5)$. This cell showed only responses to the $\mathrm{MCH}$ stimulation and no responses to the other odors tested. The responses were mainly uniformly distributed along the axonal branch, having a few boutons with a very strong response in $\gamma 4$. $\mathbf{b}$ Same as a for the magenta $\gamma$-KC from Fig. 3.4. This $\gamma$-KC showed only responses to the 3-Oct stimulation which were furthermore not uniformly distributed. This cell had only responses in $\gamma 2$ and $\gamma 3$ but none in $\gamma 4$ and $\gamma 5$. Grey bars indicate odor stimulation window. White lines indicated borders between $\gamma$-lobe compartments.

\section{3 $y$-KC-Axons are Sparse in Odor Responses}

The MB receives mainly olfactory input from olfactory projection neurons in the calyx. Here, only a small subset of $\approx 5 \%$ of $K C$ s gets activated due to odor stimulation (Honegger et al., 2011; Murthy et al., 2008; Turner et al., 2008). However, these results were drawn from calyx or cell body studies. As the MB-lobes are the main MB output region, it is important to understand how the odor signals propagate to the lobe region and if the proportion of responding KCs remains at $\approx$ $5 \%$. Furthermore, the different KC-subtypes are not differentiable in the calyx or the cell body layer. The question remains open if the sparse activation of $\approx 5 \%$ is also true for $\gamma$-KCs. Therefore, 
this study first investigated how many ${ }^{-}-\mathrm{KCS}$ respond to the monomolecular odors $\mathrm{MCH}, 3-\mathrm{Oct}$, 1Oct, as well as to their solvent mineral oil (M Oil).

The cytosolic calcium sensitive protein GCaM P3 was used to monitor the odor-evoked activity in single $\gamma$-KCs. A $\gamma$-KC was considered responsive if it was responding reliably to the odor stimulus in all imaged focal planes and its responses were greater than 3 times STD of the baseline before the odor onset.

In total it was possible to monitor odor responses in $270 \gamma$-KCs. In this set of $\gamma$-KCs 49 were reliably responding to one or more odors ( $\approx 18.15 \%)$. Only a small number of $\mathrm{\gamma}$-KCs responded to a single odor. MCH was represented by $13 \gamma$-KCs (=4.81\%), 3-Oct by $14 \gamma$-KCs $(\approx 5.19 \%), 1$-Oct by $3 \gamma$-KCs ( $\approx 1.11 \%)$, and MOil by $3 \mathrm{y}$-KCs $(\approx 1.11 \%)$. These results are comparable to previous studies showing an average number of responding KCs of $\approx 5 \%$ and in addition a response rate dependent on the odor identity (Honegger et al., 2011). In addition to $\mathrm{y}$-KCs responding to only one odor, a certain set of cells responded to more odors in various combinations (see Tab. 3.1 below). These numbers impressively represented the sparse coding of the MB also seen in other studies (Honegger et al., 2011; Turner et al., 2008).

Table 3.1: Odor response profiles of $\mathrm{y}$-KCS

\begin{tabular}{|l|c|c|}
\hline Odor response type & \# of $\boldsymbol{\gamma}$-KCs responding & \% of $\boldsymbol{\gamma}$-KCs \\
\hline $\mathrm{MCH}$ & 13 & 4.81 \\
\hline 3-Oct & 14 & 5.19 \\
\hline 1-Oct & 3 & 1.11 \\
\hline M Oil & 3 & 1.11 \\
\hline M CH/3-Oct & 3 & 1.11 \\
\hline M CH/1-Oct & 3 & 1.11 \\
\hline 3-Oct/1-Oct & 4 & 1.48 \\
\hline 3-Oct/M Oil & 2 & 0.74 \\
\hline M CH/3-Oct/ 1-Oct & 1 & 0.37 \\
\hline M CH/3-Oct/M Oil & 2 & 0.74 \\
\hline M CH/3-Oct/ 1-Oct/M Oil & 1 & 0.37 \\
\hline
\end{tabular}

$\mathrm{Y}$-KCs were assigned unreliable if they responded just once to one of the odors or responded to different odors at different imaging time points or responded outside the odor stimulus window (before or late after). In this data set 78 -KCs were found responding unreliably, leaving 143 nonresponding cells. 


\section{4 p-KC Compartments are Functional Units}

\subsection{1 $\gamma$-KCs Exhibit Highly Individual Response Profiles}

The M B is compartmentalized by the innervation of extrinsic DANs and MBONs (Aso et al., 2014a). But the question remains open regarding the functional implication this extrinsic innervation has on single $y$-KCs. Does the odor stimulus-initiated activation of $y$-KCs propagate along the entire axon or are parts of $\mathrm{y}$-KCs differently active depending on the $\mathrm{y}$-lobe compartment they are in? To address this question the single bouton responses were assigned to the $\gamma$-compartments they are part of. In this study, 31 p-KCs (from 27 flies) could be analyzed for their naïve odor responses. Here, on average, 15.67 ( \pm 5.99 STD) boutons per $\psi$-lobe compartment could be reliably identified. The $31 \gamma$-KCs showed highly individual responses. Not only did they respond to different odors but also had different predominantly active $\gamma$-lobe compartments. The pattern of these active compartments ranged from one predominant compartment to all four being uniformly active (Fig 3.5). Thereby, the number of active $\gamma$-compartments did not necessarily increase from proximal to distal compartments (starting always with $\gamma 2$ and ending with $\gamma 5$ ); there were also cases in which other combinations of $\gamma$-lobe compartments showed grouped activation. 

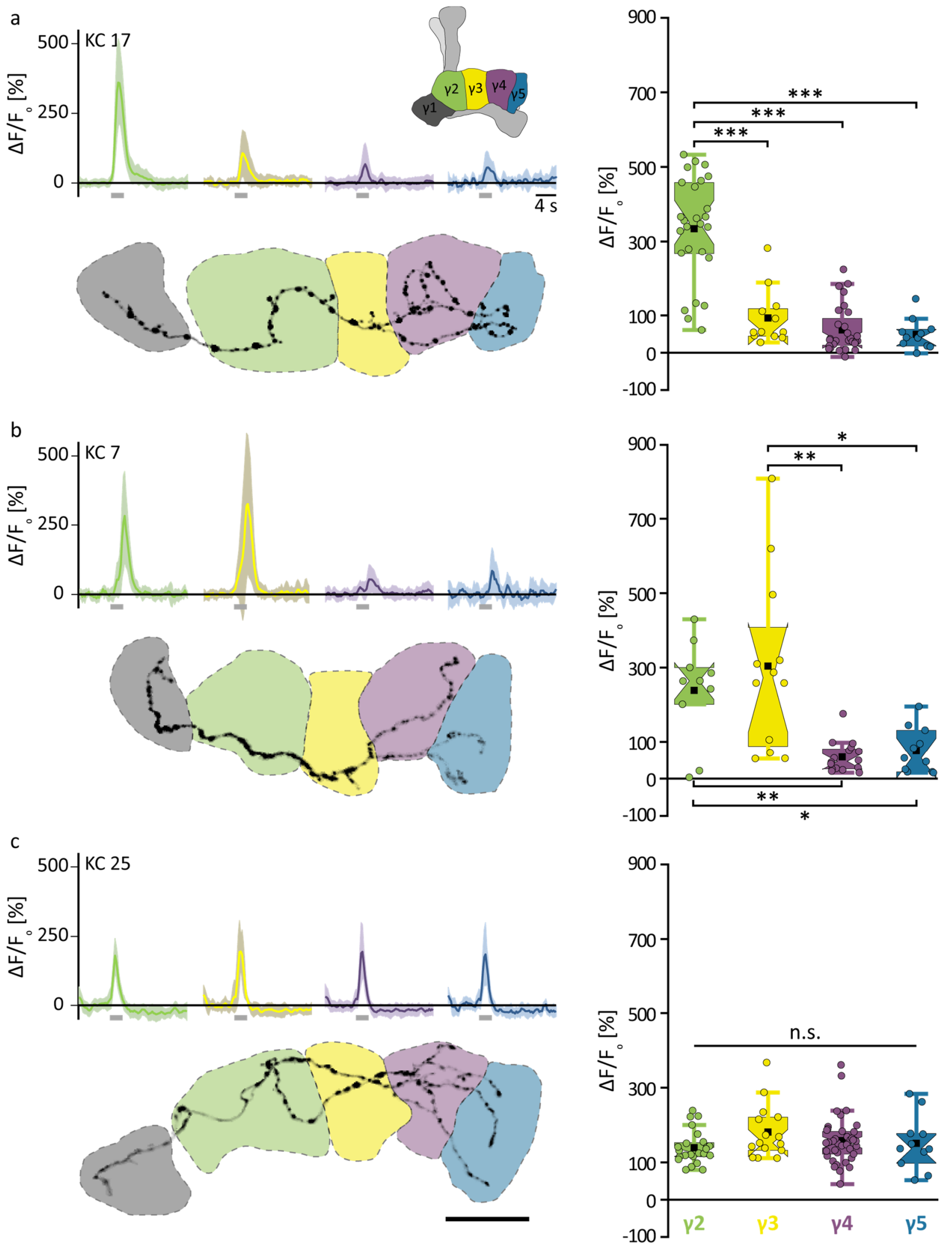

Figure 3.6: Mean responses of $\gamma$-lobe compartments in different $\mathrm{p}-\mathrm{KCS}$. a-c M ean calcium dynamics of single identified boutons to MCH stimulation in single $\psi$-KCs color coded by $\gamma$-lobe compartment as in schematic inset (upper left). The $\mathrm{\gamma}$-KC numbers correspond to position in the heat map of Fig. 3.8. Shaded areas show STD. Grey bar indicates odor stimulus window. Schematic inset in a illustrates the color code for $y$-lobe compartments used throughout the study. As boutons of the $\gamma 1$ compartment couldn't be monitored this region is left out of analysis and shaded in grey. Lower left illustrates the $\mathrm{\gamma}$-KC anatomy and its 
corresponding $y$-lobe compartments. Right box plots show the distribution of the peak response amplitudes of the boutons for each $\gamma$-lobe compartment of the respective $\gamma-K C$. a $A \gamma$-KC responding significantly stronger in the $\gamma 2$ compartment than compared to $\gamma 3-\gamma 5$. b A $\gamma-K C$ responding significantly stronger in $\gamma 2$ and $\gamma 3$ than compared to $\gamma 4$ and $\gamma 5$. C A $\gamma-K C$ responding uniformly across all $\gamma$-lobe compartments (no significant difference, n.s.). Kruskal-Wallis ANOVA was used for comparison of $\gamma$-lobe compartments and the post hoc Dunn's Test with correction for multiple comparisons. $* p \leq 0.05, * * p \leq 0.01, * * * p \leq 0.001$.

Interestingly, this phenomenon was not necessarily a cell-specific feature, because the same $\mathrm{\gamma}$-KC could show a different response pattern for another odor it was responding to as well (Fig.3.7). Figure 3.7 displays again the response pattern of $\mathrm{y}$-KC7 (see Fig. 3.6 b). This $\mathrm{y}$-KC was responding to $\mathrm{MCH}$, being predominantly active in $\gamma 2$ and $\gamma 3$ (Fig. 3.6 a). Additionally, this cell responded to 3Oct (Fig. 3.7 b), but this time being predominantly active in the distal $\gamma$-lobe compartments ( $\gamma 3$ $\mathrm{p5}$ ). Even though this was the same $\mathrm{\gamma}-\mathrm{KC}$, it showed a different response pattern that was dependent on the odor identity. For the 3-Oct stimulus the distal $\gamma$-lobe compartments $\gamma 4$ and $\gamma 5$ were significantly more active than for the MCH stimulation (Fig. 3.7 comparison between boxes in $a$ and $b$, colored asterisks).
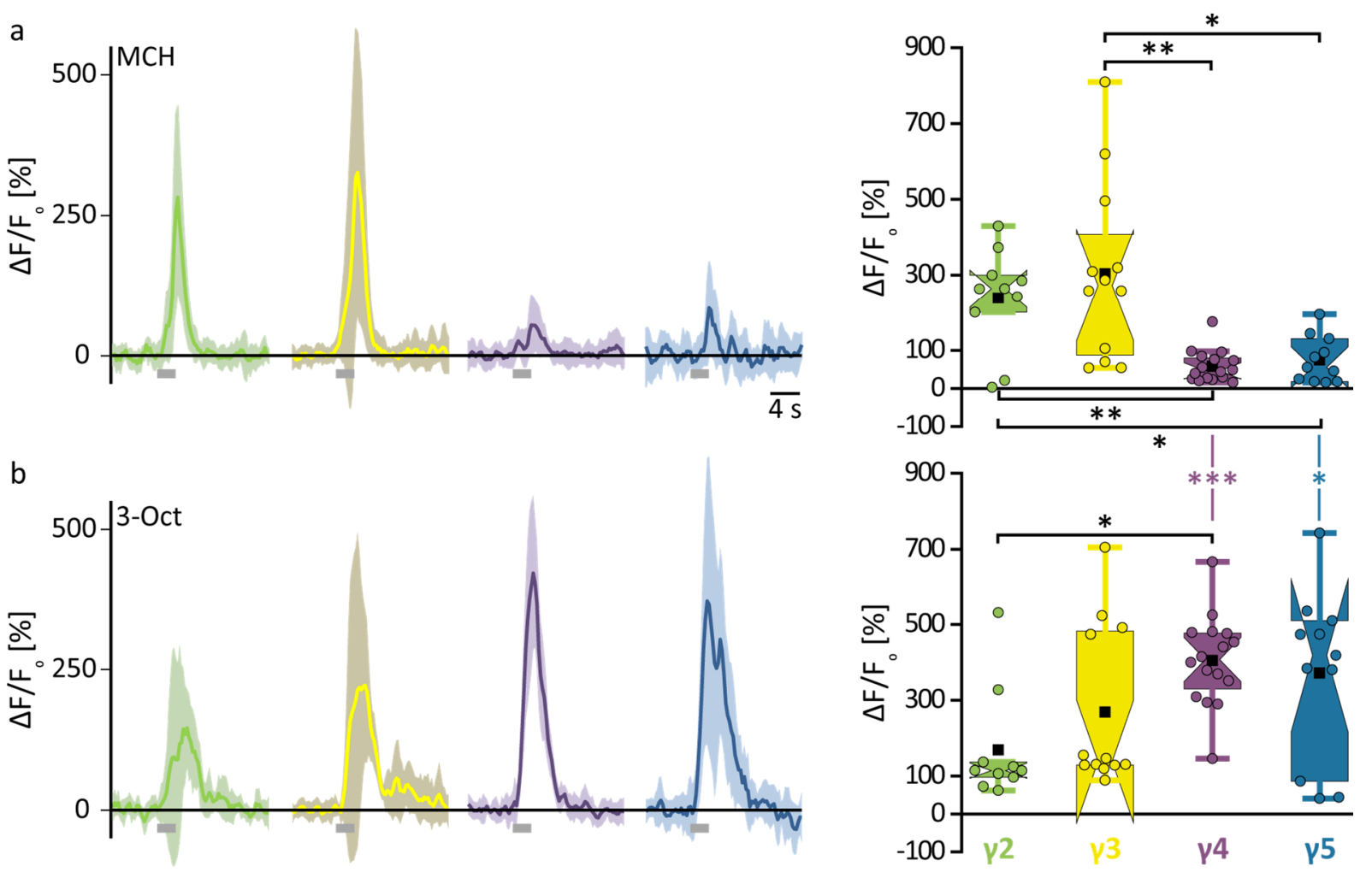

Figure 3.7: Comparison of the response profiles of the same $\mathrm{\gamma}-\mathrm{KC}(\mathrm{KC}$, Fig. $3.6 \mathrm{~b}$ ) responding to $\mathrm{MCH}$ and 3Oct stimulation. $\mathbf{a}-\mathbf{b}$ Left, mean calcium dynamics of single identified boutons. Shaded areas show STD. Grey bar indicates odor stimulus window. Right, box plots show the bouton response distribution for each $y$-lobe compartment to the respective odor stimulation. Kruskal-Wallis ANOVA was used for comparison of $\gamma$-lobe compartments and the post hoc Dunn's Test with correction for multiple comparisons. * $p \leq 0.05,{ }^{* *} p \leq$ 0.01 . The comparison of $y$-lobe compartments between $\mathbf{a}$ and $\mathbf{b}$ (colored asterisks) was done with the Paired Wilcoxon signed-rank test $(* \mathrm{p} \leq 0.05, * * * \mathrm{p} \leq 0.001)$. This $\mathrm{\gamma}-\mathrm{KC}$ is responding to $\mathrm{MCH}$ and 3 -Oct but shows different response profiles. To the $\mathrm{MCH}$ stimulation $\gamma 2$ and $\gamma 3$ are significantly more highly active than $\gamma 4$ and $\gamma 5$ (a). To the 3-Oct stimulation $\gamma 2$ is significantly less active than $\gamma 4$, and $\gamma 4$ and $\gamma 5$ are now as 
responsive as $\gamma 2$ (b). Comparing the response to both odors within a $\gamma$-lobe compartment, 3-Oct elicited a significant higher response in $\gamma 4$ and $\gamma 5$ than compared to $\mathrm{MCH}$ in the same boutons of the same $\gamma-\mathrm{KC}$ (colored asterisks).

Taken together, the responses to the given odor set were sparsely represented in the set of $\mathrm{Y}$-KC measured in this study. Those $\gamma$-KCs that responded to the odors showed a complex $\gamma$-lobe compartment specific response profile (Fig. 3.8). Furthermore, $\gamma$-KCs did not solely respond to only a single odor but showed varying combinations of odor responses (Fig. 3.8).
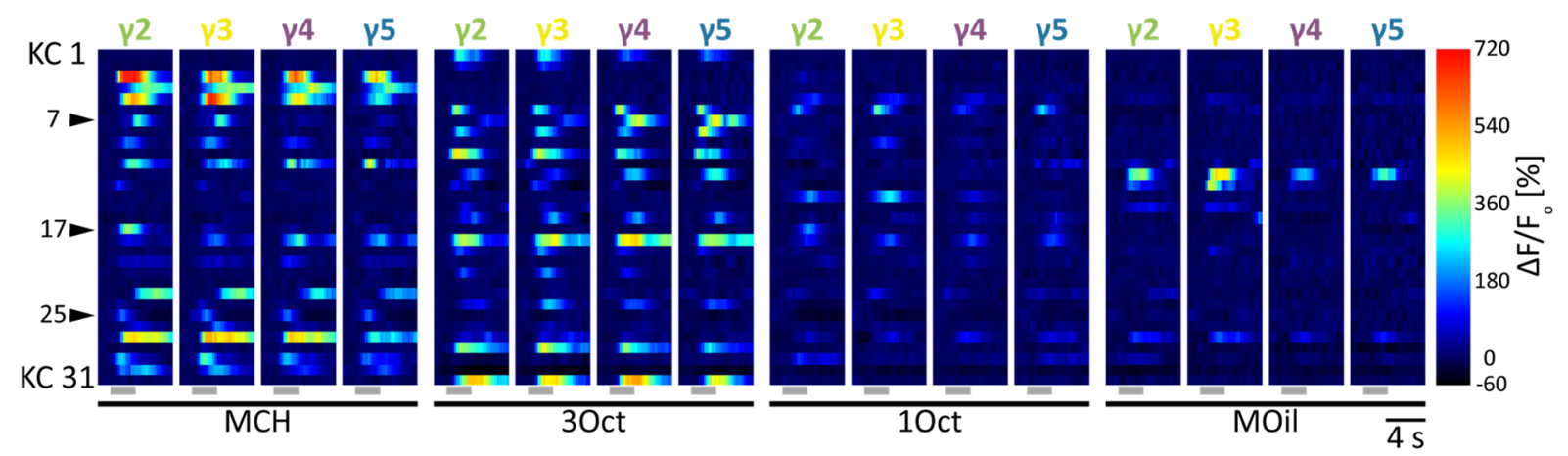

Figure 3.8: Median response profiles of $31 \mathrm{\gamma}$-KCs. Each row shows a section of the time trace ( 30 frames (7.25 s) $5 \mathrm{~s}$ after imaging onset) of the $\Delta \mathrm{F} / \mathrm{F}_{0}$ [\%] values false color coded as indicated in the calibration bar (right). Each panel corresponds to a $\mathrm{v}$-lobe compartment grouped by odor stimulation. Grey bars underneath indicate odor stimulus window. The three arrow heads on the left refer to the $\mathrm{\gamma}$-KCs in Fig. 3.6 and Fig. 3.7.

\subsubsection{A novel Similarity Analysis Demonstrates the Functional Independence of $\gamma$-Lobe Compartments}

The data has shown that $\mathrm{\gamma}$-KCs exhibit $\mathrm{\gamma}$-lobe compartment-specific profiles, but are these compartments functionally independent units? To address this question a new analysis was introduced (see 2.2.7). This analysis compared the similarities between $\gamma$-lobe compartments. First, the peak responses (average of 5 time frames, see Methods 2.2.6) for each of the 1945 boutons were calculated. To compare the activity between the $\gamma$-lobe compartments, the median of each responding compartment was calculated. This resulted in 45 odor responses per $\mathrm{y}$-lobe compartment. Even though each $\mathrm{\gamma}-\mathrm{KC}$ responded individually along $\mathrm{\gamma}$-lobe compartments, the median responses showed no differences between compartments (Fig. 3.9 a). Only a slight tendency was observed for $\gamma 4$ and $\gamma 5$ showing weaker responses. Therefore, the second step was to calculate the normalized cross-correlation coefficient between the boutons of a $y-K C$ within (internal) a compartment and between (external) compartments (Fig. 3.9 b). Boutons that showed responses to an odor were highly correlated even though their peak intensities varied in magnitude (Fig. 3.9 b). To compare the correlations between $\gamma$-lobe compartments, the median 
correlation coefficient was calculated for each compartment in each $\gamma-K C$. Here as well, the compartments that were responding showed high correlations and did not reflect the differences in amplitude (Fig 3.8 e). To combine both features of the bouton responses and compare the similarities of $y$-lobe compartments the cross-correlation coefficients of each bouton pair was multiplied by the amplitude contrast of the respective bouton pair (see methods 2.2.7). The bouton responses were subsequently normalized to the maximum of the response, to be able to compare the values between $\mathrm{\gamma}$-KCs. This amplitude corrected correlation (ACC) index reflected the pattern of similarity within the $\gamma$-lobe compartment (Fig. $3.9 \mathrm{f}$, highlighted diagonal matrix fields) and between compartments (Fig. 3.9 f, remaining matrix fields).
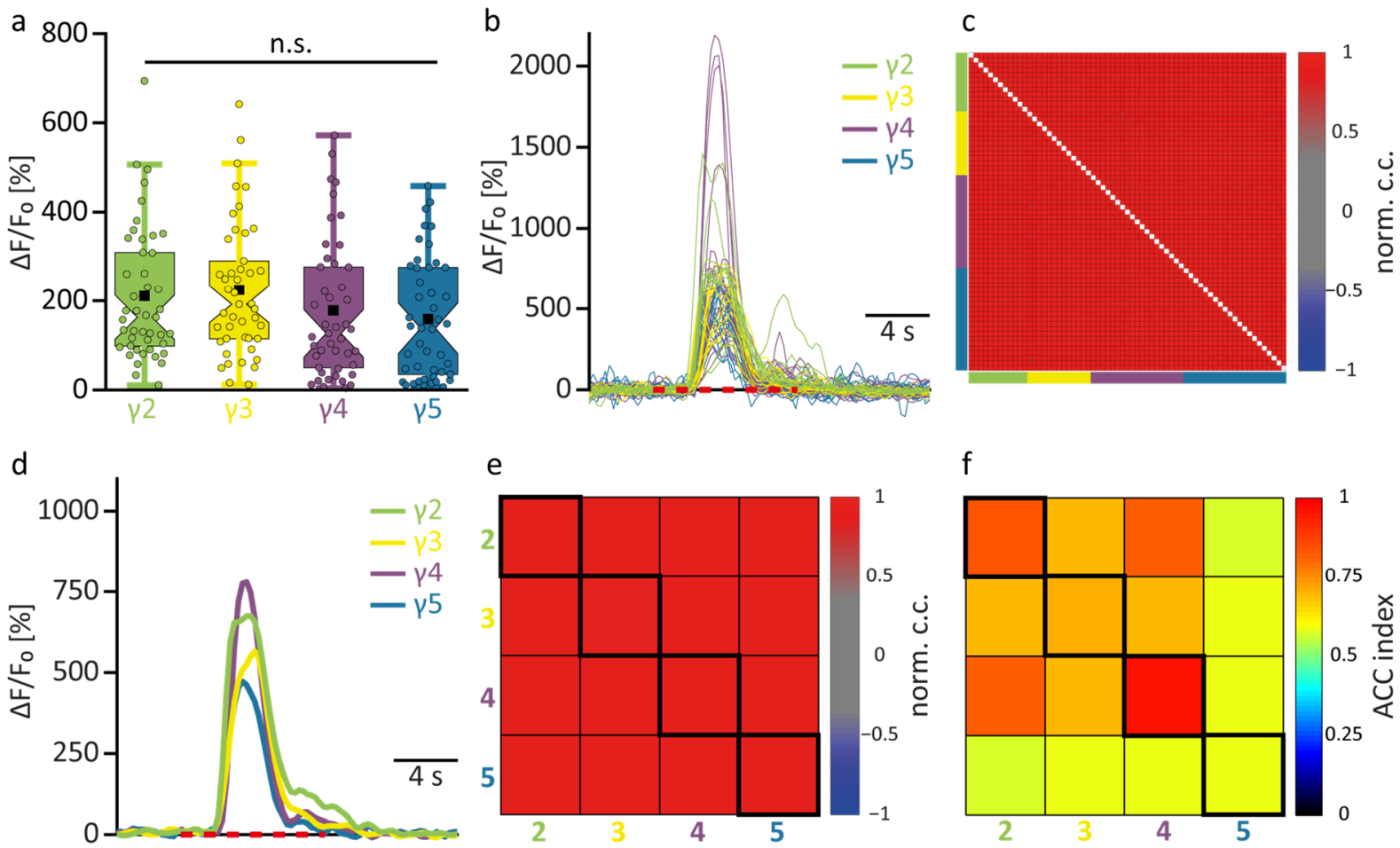

e

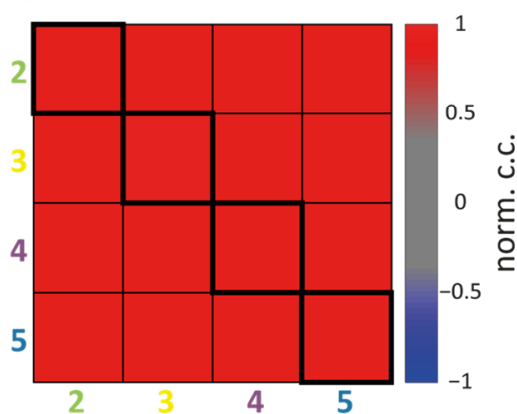

f

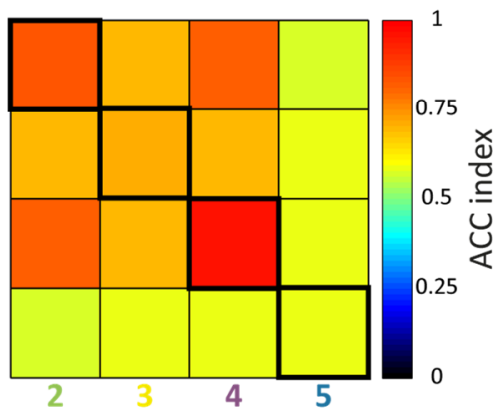

Figure 3.9: Activity corrected correlation as a new measure for similarity. a Box-plot shows the 45 median responses of the $31 \mathrm{\gamma}$-KCS. No significant difference (n. s.) was found between the $\gamma$-lobe compartments. There is a tendency for $\gamma 4$ and $\gamma 5$ being lower in intensity than $\gamma 2$ and $\gamma 3$. b Single bouton calcium dynamics of an exemplary $\mathrm{y}-\mathrm{KC}(\mathrm{KCl}$, yellow, in Fig. 3.4) responding to $\mathrm{MCH}$ stimulation. Colored time traces correspond to the respective $\gamma$-lobe compartment. Different boutons responded in different intensities to the same stimulation. Red dashed line indicates the correlation window that was used for further analysis. c M atrix showing the normalized cross-correlation coefficient (norm. c.c.) of the time traces of each bouton in b. As all boutons showed an increase in intensity, the norm. c.c. were high (red). The norm. c.c. could not reflect the differences in the peak intensities (b). Colored bars left and beneath the matrix indicate which boutons belong to which $\gamma$-lobe compartment. $\mathbf{d}$ Median calcium dynamics of $\mathbf{b}$ for the four $\gamma$-lobe compartments. On average all compartments showed a response to the $\mathrm{MCH}$ stimulation with different peak intensities. e Median norm. C.c. of c plotted in a matrix showing the $\gamma$-lobe compartments' internal correlation on the diagonal (highlighted in thick black contours) and the correlations between compartments. Here as well, the differences in peak intensities (d) could not be reflected. $\mathbf{f}$ After introduction of the amplitude corrected correlation (ACC, see methods 2.2.7) index the matrix could reflect the strength of similarities (color map from black to red). 
With the ACC index it was now possible to visualize the relationships between the $y$-lobe compartments and address the question if they are functionally independent units. First, the medians of all ACC indices were plotted in a colored matrix (Fig. 3.10 a, values in Tab. 3.2). This matrix showed that the naïve odor responses, of the $31 \mathrm{\gamma}$-KCs (45 odor responses) measured, were more similar within a compartment than between them. Specifically, $\gamma 2$ and $\gamma 3$ showed the highest internal similarities (yellow ACC index), thereby grouping together. The $\mathrm{v}$-lobe compartments 4 and 5 had lower internal similarities (cyan ACC index), thereby also grouping together. This tendency was comparable to the trend in the median bouton fluorescence intensities (Fig. 3.9 a). In order to determine the functional independence of the $\gamma$-lobe compartments, the contrast of the $y$-lobe internal ACC index to the external ACC indices was calculated. If the median contrast would have resulted in 0 there would have been no difference between the internal and the external similarities. This would have meant that the boutons of one $\gamma$-lobe compartment were not distinguishable from boutons of other compartments. For $\gamma 2-\gamma 4$ the $95 \% \mathrm{Cl}$ of the median ACC index contrasts were above the 0 -value. Therefore, it could be demonstrated that the $\mathrm{\gamma}^{2-}, \mathrm{\gamma} 3-$, and $\mathrm{\gamma}^{4}$-compartment can be assumed to be functionally

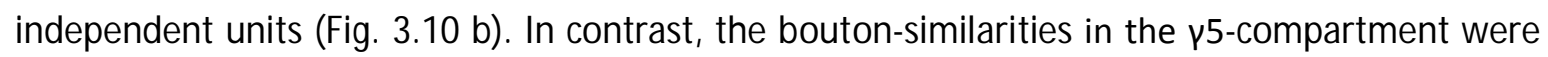
not distinguishable from boutons of other $\gamma$-lobe compartments. This finding demonstrates for the first time that $\gamma$-KCs are not binarily coding for an odor by simply being active or not active. They comprise functional units that could be independently active and might code for different modalities of a stimulus. This finding demonstrates that the number of coding combinations is increased by the number of $\gamma$-lobe compartments. Hence, it increases the odor coding space enormously.
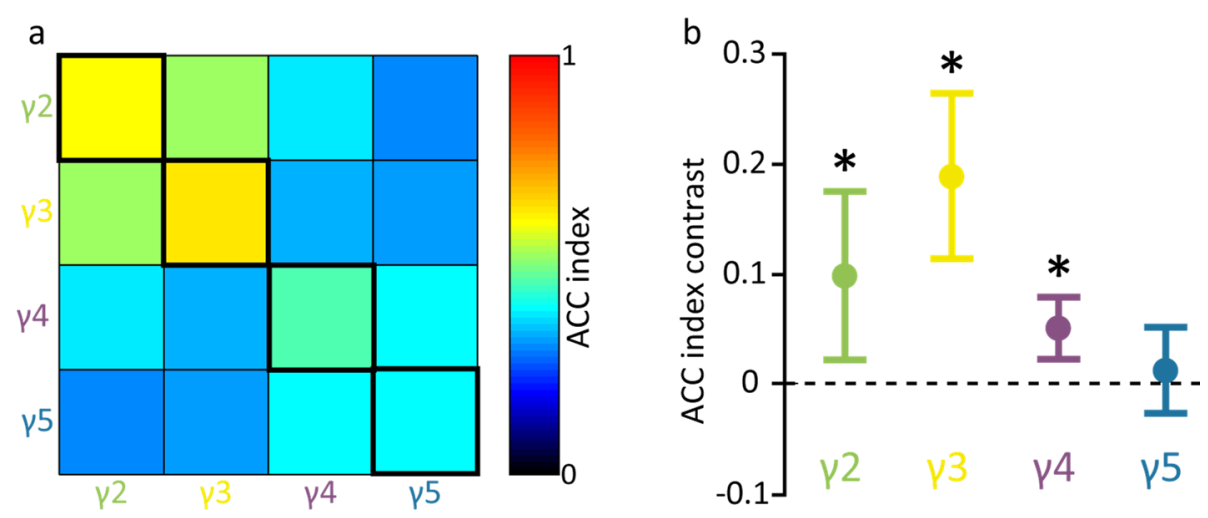

Figure 3.10: $\gamma$-lobe compartments are functionally independent units. a M edian similarity (ACC index) of all $31 \mathrm{v}$-KCs illustrated as color coded matrix (values in Tab. 3.2). Diagonal indicates the internal similarity (highlighted as black contour). Remaining fields indicate the external similarities between $\gamma$-lobe compartments. b Contrast of the internal ACC index to the external ACC index. The circles indicate the median ACC index contrast; whiskers indicate the $95 \% \mathrm{Cl}$. The Cls of $\gamma 2-\gamma 4$ do not cross the 0 -value, resulting in a $5 \%$ significance being different from 0 . 
Table 3.2: Median ACC indices of the 31 y-KCs plotted in Fig. 3.10

\begin{tabular}{|c|c|c|c|c|}
\hline & ү2 & v3 & v4 & ү5 \\
\hline ү2 & 0.60431 & 0.5233 & 0.38328 & 0.30769 \\
\hline ү3 & 0.5233 & 0.60462 & 0.34005 & 0.31706 \\
\hline ү4 & 0.38328 & 0.34005 & 0.46602 & 0.39657 \\
\hline p5 & 0.30769 & 0.31706 & 0.39657 & 0.4014 \\
\hline
\end{tabular}

\subsection{Olfactory Associative Training Changes the Odor Code}

The coincidence of a conditioned stimulus (CS) with an unconditioned stimulus (US) is thought to change the synaptic strength (Kahsai and Zars, 2011). Here, an odor (CS+) was paired with an electric shock (US) and the question arises: What changes in the course of aversive olfactory associative learning? To address this question 18 flies (20 $y$-KCs, 24 odor responses) were subjected to a reciprocal aversive olfactory learning paradigm under the microscope (see methods 2.2.3). As $y$-KCs did not respond to all odors, each odor response was decided to be trained as CS+ or CS- to balance the number of conditions (CS+: $4 \times \mathrm{M} \mathrm{CH}+4 \times 3-0 \mathrm{ct}=8 \times \mathrm{CS}+$ responses; CS-: $4 \times 3$ $\mathrm{Oct}+4 \mathrm{xMCH}=8 \mathrm{x}$ CS- responses). Three of these $\mathrm{y}$-KCs responded to both odors. In these cases $\mathrm{CS}+$ and CS- could be monitored simultaneously (1 $y-\mathrm{KC}$ : CS $+=\mathrm{MCH}, \mathrm{CS}-=3-0 \mathrm{ct} ; 2 \mathrm{y}-\mathrm{KCs}$ : CS $+=3-$ Oct, $\mathrm{CS}-\mathrm{=}$ MCH). In the control condition the same protocol was applied but without presenting a shock and the order of the presented odors was changed reciprocally (CS1: $2 x \mathrm{M} \mathrm{CH}+2 \times 3-0 \mathrm{ct}$; CS2: $2 \times 3-0 \mathrm{ct}+2 \times \mathrm{MCH}=8 \times$ control responses). One of the control $y-\mathrm{KC}$ responded to both odors $(\mathrm{CS} 1=\mathrm{MCH}, \mathrm{CS} 2=3-0 \mathrm{ct})$. 

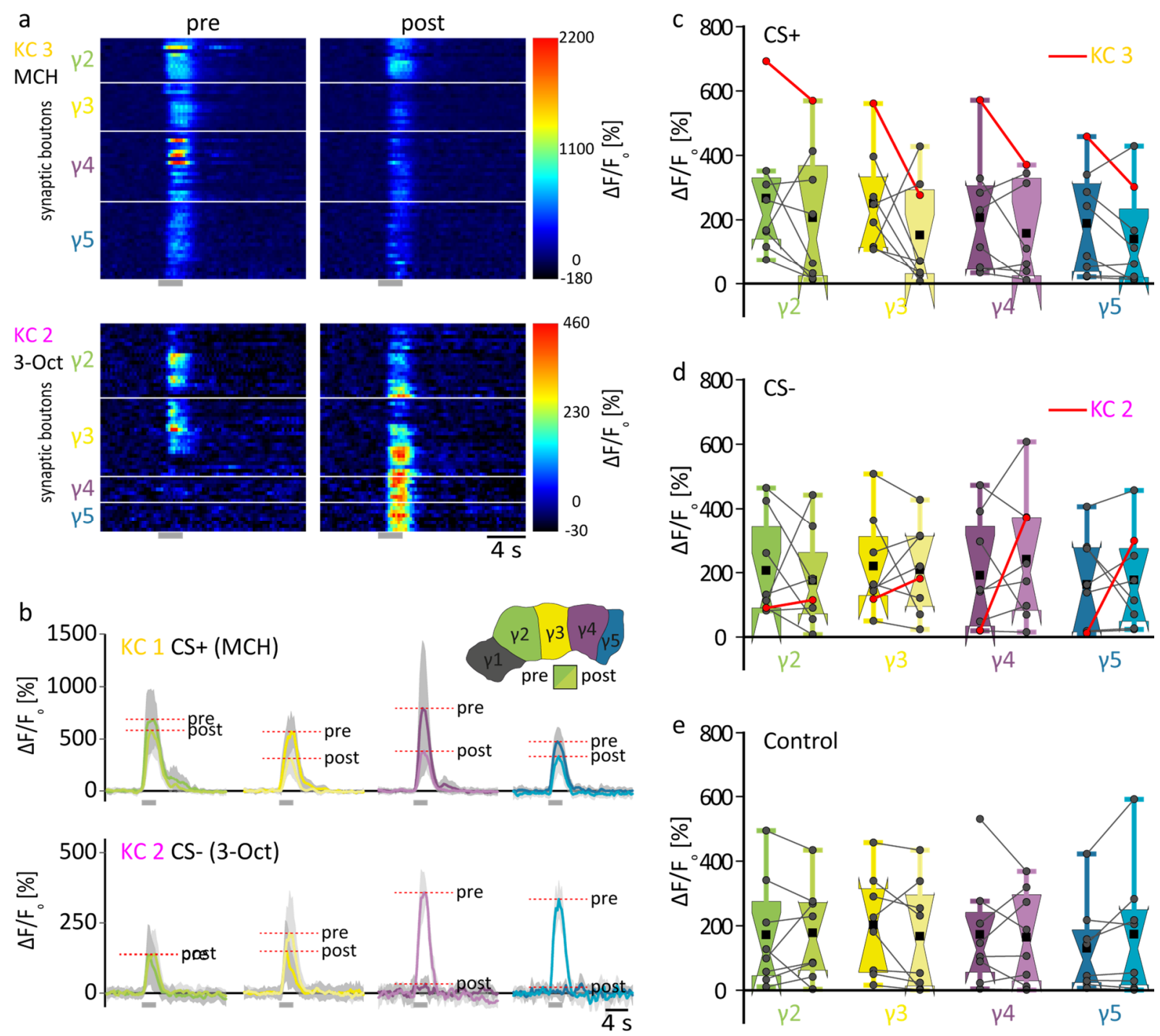

Figure 3.11: Fluorescence intensities for all trained $\gamma-K C s$. a False color coded heat map of the two exemplary $\gamma$-KCS KC1 (top) and KC2 (bottom, see Fig. 3.4). Each row shows the time trace of the single boutons sorted by $\gamma$-lobe compartment for MCH stimulation (CS+, KC1) and 3-0ct stimulation (CS-, KC2) before (pre) and after the training (post). Grey bars indicate odor stimulus window. b Mean calcium dynamics of the same exemplary $\gamma$-KCs (a) showing the change in the response intensities for each $\gamma$-lobe compartment. Schematic inset indicates $\psi$-lobe compartment color code as before. Post condition is colored in respective lighter colors. Shaded areas indicate the STD of the mean traces. Grey bars indicate odor stimulation window. c-e Box plot pairs showing the median response distribution for all odor responses for the three training conditions ( $\mathbf{c}-\mathrm{CS}+\mathbf{d} \mathbf{d}$ - CS- and $\mathbf{e}$ - control; $\mathrm{n}=8$ for each condition). In each pair the left box indicates the pre responses (darker color) and the right box the post responses (lighter color). For each individual odor response the pre and post data point are connected with a grey line. Red lines correspond to the respective exemplary $\mathrm{\gamma}$-KCs of $\mathbf{a}$ and $\mathbf{b}$. All graphs show highly variable changes with no significant difference between groups (paired Wilcoxon signed rank test).

In order to investigate the effect of aversive olfactory associative learning, the medians of all time frames across the boutons of each $\gamma$-lobe compartment were calculated resulting in median time traces (Fig. 3.11 b). In these time traces the average peak responses were determined (see methods 2.2.6). These peak responses of each $\mathrm{y}$-lobe compartment for each $\mathrm{\gamma}$-KC were compared to detect fluorescence intensity changes that might occur after the training (Fig. $3.11 \mathrm{c}$ ). 
Comparing the individual $\mathrm{\psi}-\mathrm{KCS}$, no consistent depression or facilitation was observed. Boutons rather showed individual changes due to the associative training independent of training condition (CS+, CS- or control). Even though individual p-KCs show diverse changes (Fig. 3.11 c-e) in the course of aversive olfactory associative training, no significant changes between the pre and post condition in $y$-lobe compartments in the different training conditions could be detected. Nevertheless, there was a trend in the mean and median responses to decrease after the training in the CS+(Fig. 3.11 c, black squares and notches, respectively).
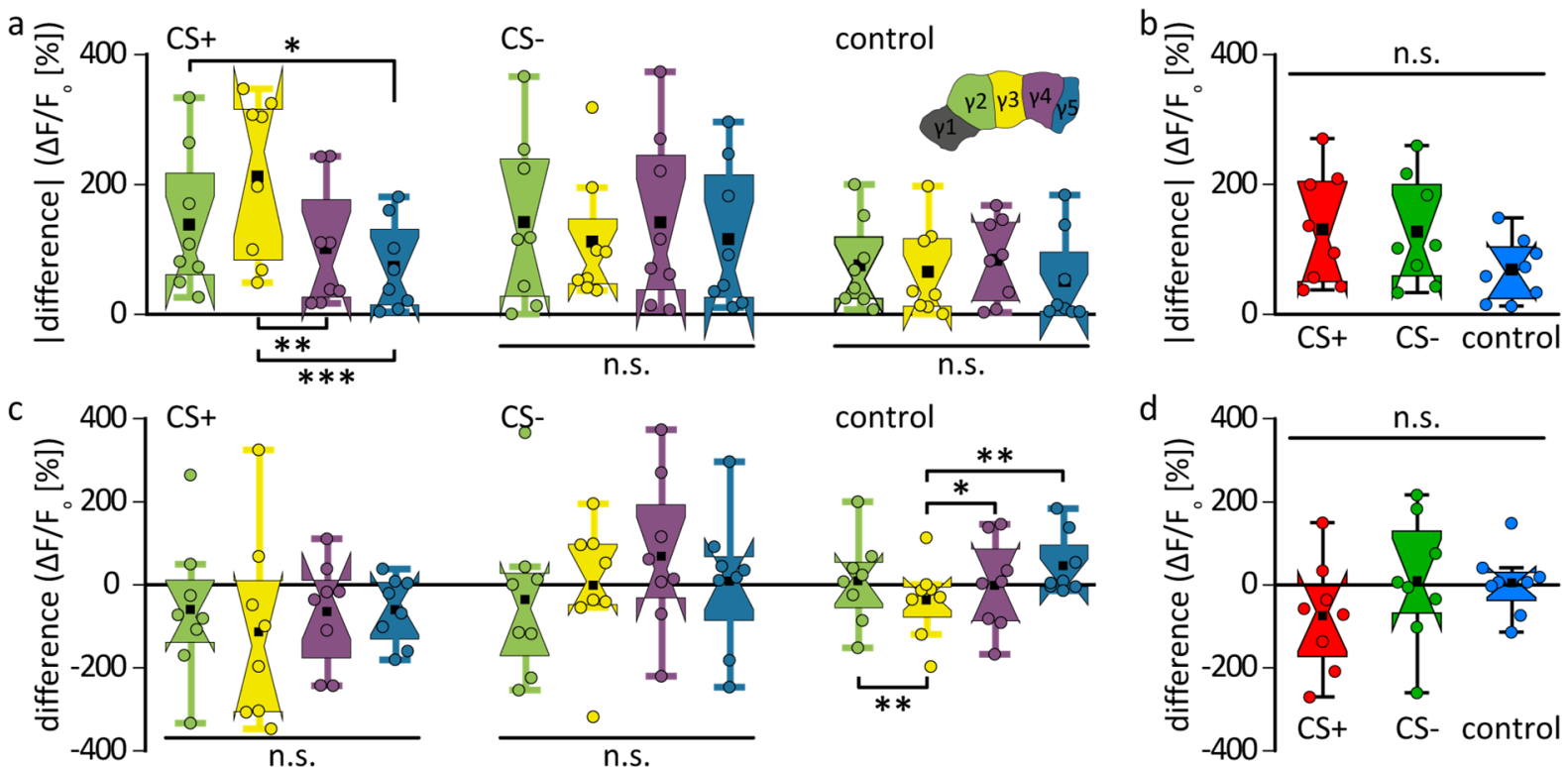

Figure 3.12: Differences in amplitudes after the training. a Absolute differences (| post - pre|) for the three training conditions for all $\gamma$-lobe compartments. Only in the CS+ condition $\gamma 2$ and $\gamma 3$ were significantly different from $\gamma 4$ and $\gamma 5$. In the other two training conditions no significance was detected (n.s., p >0.05; *, $\mathrm{p}<0.05 ; * *, \mathrm{p}<0.01 ; * * *, \mathrm{p}<0.001$; Friedman-ANOVA). b Mean absolute difference (of a) across $\gamma$-lobe compartments in the three training conditions showing no difference between the groups (n.s., $p>0.05$, one-way-ANOVA). c Difference occurring after the training (post - pre) for the three training conditions for all $p$-lobe compartments. None of the groups were significantly different from $0(p>0.05$, Wilcoxon signed rank test against 0 ). In the CS+ and CS- conditions the $\gamma$-lobe compartments are not significantly different from each other. Only in the control condition $\mathrm{\gamma} 3$ was significantly different from the other compartments (n.s., $*, p<0.05 ; * *, p<0.01$, Friedman-ANOVA). All statistics were corrected with Benjamini Hochberg FDR correction. $\mathbf{d}$ Mean difference (of $\mathbf{c}$ ) across $\mathrm{y}$-lobe compartments in the three training conditions showing no difference between the groups (n.S., $p>0.05$, one-way-ANOVA).

To further investigate if synaptic plasticity occurred in the course of olfactory associative learning, first the absolute difference (| post - pre|) in the median peak calcium intensities of each $\gamma$-lobe compartment in each $\mathrm{y}$-KC was calculated (Fig. $3.12 \mathrm{a}$ ). Here, only in the CS+ condition changes occurred in the two proximal $\gamma$-lobe compartments ( $\gamma 2$ and $\gamma 3$ ). Both showed significantly more changes after the training than $\gamma 4$ and $\gamma 5$ did. In the CS- condition changes occurred as well but uniformly across $\gamma$-lobe compartments. In the control condition changes were less drastic than seen in the other conditions. To compare the overall effect in the three training conditions, the 
mean across $\psi$-lobe compartments was calculated for each $\psi$-KC (Fig. 3.12 b). This showed that the changes in the CS+ and CS- conditions were equally strong, with the tendency in both to be greater than in the control condition.

To examine in which direction the calcium intensities changed after training, the difference (post - pre) was calculated for each $\gamma$-lobe compartment (Fig. 3.12. c). No significant change to 0 was found in any of the three training conditions, although the trend in the CS+ condition is again noticeable. Comparing the training effect across $y$-lobe compartments only in the control condition, $\gamma 3$ was significantly different from the other $\gamma$-lobe compartments. The average effect across $\mathrm{\gamma}$-KCS (Fig. $3.12 \mathrm{~d}$ ) indicated a trend where the calcium activity decreased in the CS+ condition after the training.

Comparing the time courses of the calcium dynamics before and after the training (Fig.3.11, heat maps), the shapes of individual time traces were also changed in the course of associative training. For example, in one $\mathrm{\gamma}$-KC (Fig. 3.13 a, odor response $4=$ or 4 ), which was trained as CS+, the time interval between odor onset and peak response was reduced in $\gamma 2$ and $\gamma 3$ after the training but slightly increased in $\gamma 4$ and $\gamma 5$ in the same $\gamma-K C$. In another $\gamma-K C$ that was trained as CS- (Fig. 3.13 b, or 11) the time interval between odor onset and peak response was reduced after the training in $\gamma 2$ and $\gamma 3$ but did not change in $\gamma 4$ and $\gamma 5$. In a second $\gamma-\mathrm{KC}$ that was trained as CS(Fig. 3.13 b, or16), the time interval between odor onset and peak response was extended after the training in in all $\mathrm{y}$-lobe compartments. Furthermore, the response dynamic became more transient by reducing the decay time drastically after the training. An extension of the time interval between odor onset and peak response could be detected as well in the $\gamma 3$ compartment in the control condition for several $\gamma$-KCs (Fig. $3.13 \mathrm{C}$ ). Nevertheless, the median calcium dynamics of the three training conditions in the four $y$-lobe compartments showed no difference between pre and post training (Fig. 3.13, lower panels). There was a slight tendency in the CS+condition that the median calcium dynamics decreased after the training. This effect can also be seen when the median differences were plotted (Fig. 3.12). 

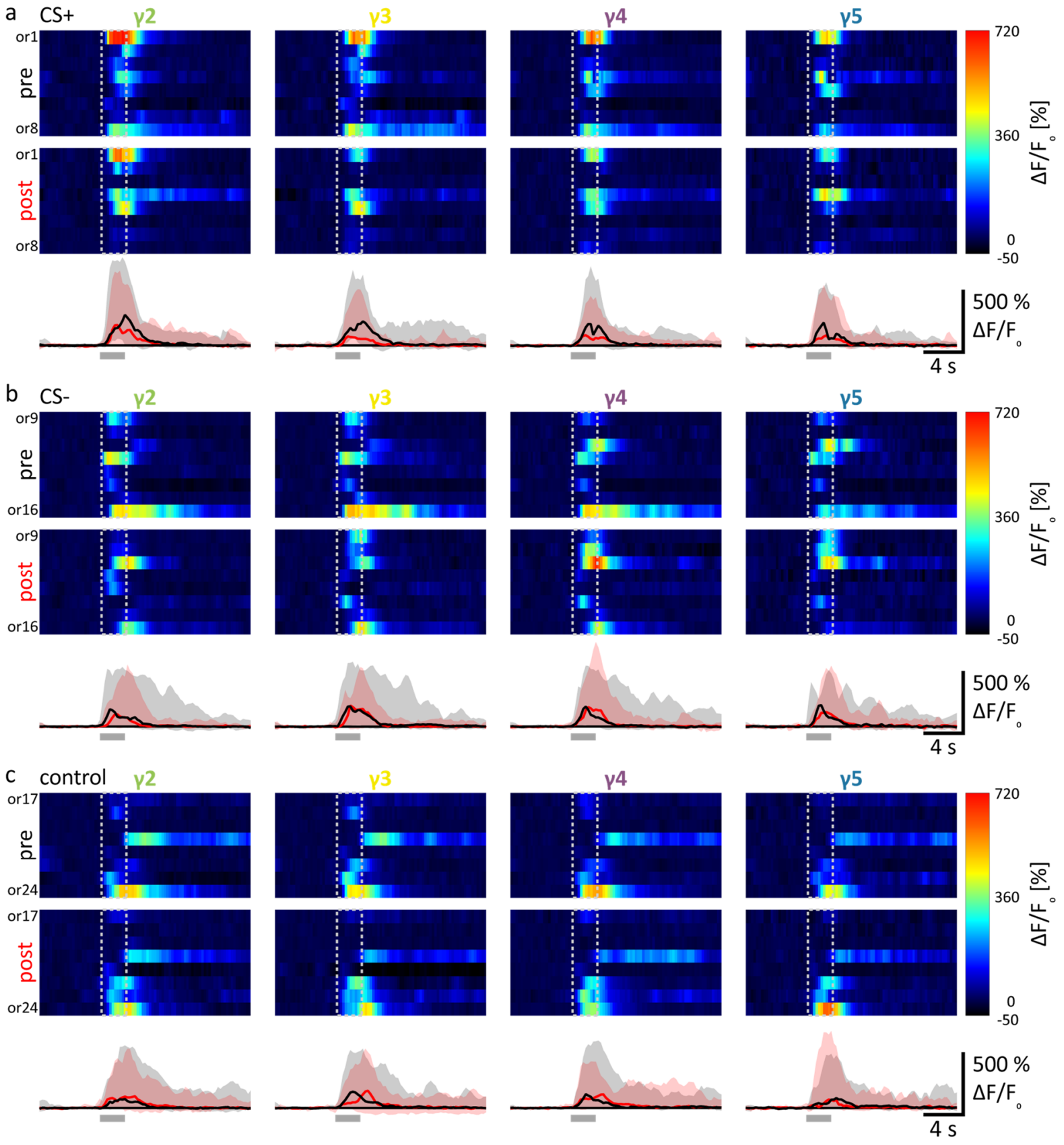

Figure 3.13: Median responses for the 24 odor responses before and after the training. a-c Upper panels show median response traces for each $\psi$-lobe compartment $\left(\gamma_{2}-\gamma 5\right)$ as false color coded heat maps. Each row corresponds to an odor response (or1-24) before (pre) and after (post) the training for the CS+(a), CS(b), and control (c) condition. Lower panel shows the respective median trace (shaded area indicates $95 \%$ $\mathrm{Cl}$ ) before (black) and after (red) the training. Dotted white boxes and grey bars indicate odor stimulus window.

As the mere intensities of the $\mathrm{y}$-KC boutons did not show a significant change in the course of aversive olfactory associative learning and the calcium dynamics indicated a temporal change, the same similarity analysis (as for the pre analysis, see 3.4.2) was employed for the training groups.

To quantify the indications of changes in the calcium dynamics (Fig. 3.13), first, the normalized cross-correlation coefficients of all 1457 boutons of the 20 trained $\mathrm{y}$-KCs and the respective 
medians were calculated (Fig. 3.14 a, values of colored matrices in Tab. 3.3). In order to determine the overall change due to the associative training the coefficients before the training of all three training conditions were pooled and the median internal and external cross-correlation coefficients were plotted in a color coded matrix (Fig. 3.14, left, values in Tab. 3.3). Here, similar to the pre-only analysis (Fig. 3.10), ${ }^{2}-\nu_{4}$ group together showing more comparable correlation coefficients. Attenuated, $\gamma 5$ grouped more with $\gamma 4$, being more different to $\gamma 2$ and $\gamma 3$. The median internal and external cross-correlation coefficients after the training were also calculated for all three training conditions (Fig. 3.14, middle column). The post-training coefficients were subtracted from the pre-training coefficients to calculate the differences induced by associative training (Fig. 3.14 right column). The CS+ condition showed strong decorrelations within the $\gamma^{-}$ lobe compartments, as well as between them. Specifically, the correlation between $\gamma 2$ and $\gamma 3$ decreased significantly, ungrouping these compartments. No changes were detected in the CScondition. Interestingly, there was a strong, though not significant, decorrelation within $\gamma 5$ in the control condition and therefore a decorrelation between $\gamma 5$ and the other $\gamma$-lobe compartments. This finding indicates that aversive olfactory associative training decorrelates bouton responses within and across $y$-lobe compartments after pairing an odor (CS+) with an electric shock. On the contrary, the non-shocked odor (CS-) does not change in the course of associative training. The prolonged presentation of odors without electric shock presentation in the protocol seems to decorrelate the bouton responses within the ${ }^{5}$ compartment, inducing more variable responses within this compartment.

Neurons do not just simply integrate separate response features - like amplitude or onset - from pre-synaptic neurons, but complex signals as a combination of both (Stuart and Spruston, 2015). Therefore, in the next step the correlations were corrected for their amplitudes (see methods 2.2.7) and the ACC indices calculated (Fig. 3.14 b, values of colored matrices in Tab. 3.4). In the pre-training condition the $\gamma 3$ compartment showed the highest internal similarity and grouped with $\gamma 2$ and $\gamma 4$ (Fig. 3.14 b, left column). The $\gamma 5$ compartment had the lowest internal similarity. These findings are in line with the previous pre-only analysis with all $\gamma$-KCs (Fig. 3.10). After the training, a drastic and significant decrease in the ACC indices was observed in the CS+ condition. Only the internal similarity of $\gamma 2$ and the similarity between $\gamma 3$ and $\gamma 5$ did not decrease significantly (Fig. 11 b, right column, upper matrix). These findings indicate that the associative training induced a strong dissimilarity and therefore desynchronization within and between $\gamma$-lobe compartments. No such change was observed in the CS- or the control condition. On the contrary, in the CS- condition a light increase in the internal similarity of $\gamma 4$ and $\gamma 5$ and a strong increase between $\gamma 3$ and $\gamma 5$ was detected, though not significant. Interestingly, this increase between $\gamma 3$ and $\gamma 5$ fits complementarily into the non-significant field in the CS+condition. Furthermore, the 
CS- conditioning led to a grouping of $\gamma 3-\gamma 5$, reducing the similarity to $\gamma 2$ that is the opposite effect seen in the pre-condition.

a

correlation

\section{post training}

CS+

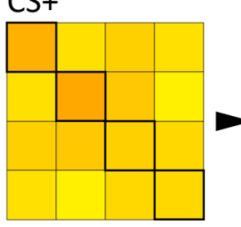

CS-

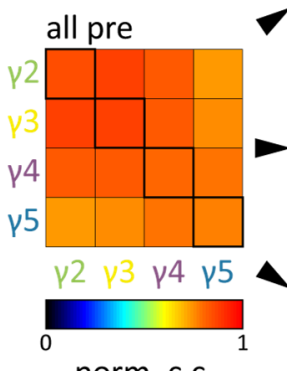

norm. c.c.



control

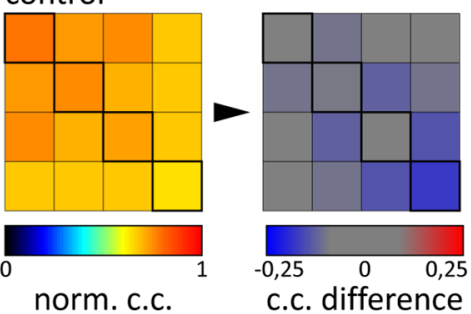

b

similarity

post training

post - pre

$\mathrm{CS}+$

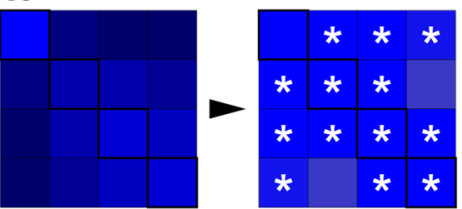

CS-
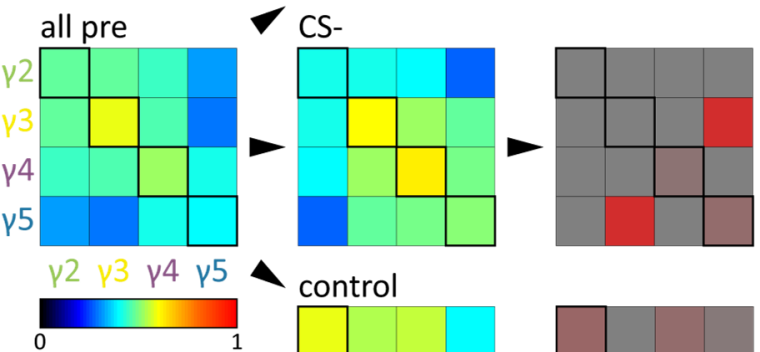

ACC index

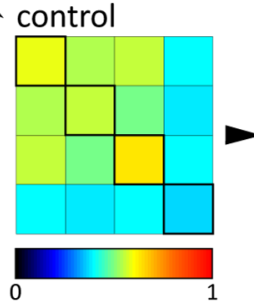

ACC index

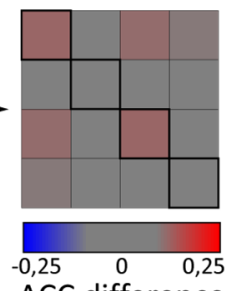

ACC difference

Figure 3.14: The odor response pattern changes in the course of associative training. a Correlation matrices showing the median internal (diagonal, highlighted in black contours) and external normalized crosscorrelation coefficients (norm. c.c.) as color coded maps (values in Tab. 3.3). All 24 pre-training odor responses were pooled (left). Middle column shows the norm. c.c. after the training. Right column shows the difference of the norm. c.c. after the training (pre subtracted from post). Asterisks indicate $5 \%$ significance for $95 \% \mathrm{Cl}$ being different from 0 . b Similarity matrices showing the median internal and external ACC indices (values in Tab. 3.4) on the left - pre-condition, middle - post-conditions, right difference post - pre (as in a). Asterisks indicate $5 \%$ significance for $95 \% \mathrm{Cl}$ being different from 0 . Internal similarity is highlighted in black contours.

In summary, no uniform depression or facilitation was found in the course of aversive olfactory associative training. Rather, the naïve $\gamma$-lobe compartment-specific odor code was altered in a way that the $\gamma$-lobe compartments became dissimilar within and between each other when paired with an electric shock. This demonstrates a new form of synaptic plasticity: a desynchronization of synaptic odor representations. 
Results

Tables 3.3: Median correlation coefficients of the training conditions plotted in Fig. $3.14 \mathrm{a}$

\begin{tabular}{|c|c|c|c|c|}
\hline pre & v2 & ү3 & v4 & v5 \\
\hline ү2 & 0.9006 & 0.9086 & 0.8769 & 0.7991 \\
\hline ү3 & 0.9086 & 0.9173 & 0.8852 & 0.7986 \\
\hline v4 & 0.8769 & 0.8852 & 0.8667 & 0.8520 \\
\hline ү5 & 0.7991 & 0.7986 & 0.8520 & 0.8350 \\
\hline
\end{tabular}

\begin{tabular}{|c|c|c|c|c|}
\hline CS+ & v2 & v3 & v4 & v5 \\
\hline v2 & 0.7543 & 0.6687 & 0.6930 & 0.6675 \\
\hline v3 & 0.6687 & 0.7701 & 0.7094 & 0.6386 \\
\hline v4 & 0.6930 & 0.7094 & 0.6878 & 0.6826 \\
\hline v5 & 0.6675 & 0.6386 & 0.6826 & 0.6829 \\
\hline
\end{tabular}

\begin{tabular}{|c|c|c|c|c|}
\hline CS- & v2 & v3 & v4 & v5 \\
\hline ү2 & 0.8622 & 0.8984 & 0.8573 & 0.8518 \\
\hline ү3 & 0.8984 & 0.9137 & 0.9255 & 0.8872 \\
\hline v4 & 0.8573 & 0.9255 & 0.8974 & 0.8836 \\
\hline v5 & 0.8518 & 0.8872 & 0.8836 & 0.8784 \\
\hline
\end{tabular}

\begin{tabular}{|c|c|c|c|c|}
\hline Ctrl & v2 & v3 & v4 & v5 \\
\hline v2 & 0.8504 & 0.8070 & 0.8145 & 0.7176 \\
\hline v3 & 0.8070 & 0.8260 & 0.7624 & 0.7089 \\
\hline v4 & 0.8145 & 0.7624 & 0.7955 & 0.7100 \\
\hline v5 & 0.7176 & 0.7089 & 0.7100 & 0.6617 \\
\hline
\end{tabular}

Tables 3.4: Median ACC indices of the training conditions plotted in Fig. $3.14 \mathrm{~b}$

\begin{tabular}{|c|c|c|c|c|}
\hline pre & $v^{2}$ & p3 & v4 & p5 \\
\hline$\gamma^{2}$ & 0.4699 & 0.4763 & 0.4443 & 0.3126 \\
\hline$\gamma 3$ & 0.4763 & 0.5639 & 0.4575 & 0.2922 \\
\hline p4 & 0.4443 & 0.4575 & 0.5256 & 0.4074 \\
\hline p5 & 0.3126 & 0.2922 & 0.4074 & 0.4008 \\
\hline
\end{tabular}

\begin{tabular}{|c|c|c|c|c|}
\hline CS+ & v2 & v3 & v4 & v5 \\
\hline v2 & 0.1921 & 0.0967 & 0.0929 & 0.0876 \\
\hline v3 & 0.0967 & 0.1356 & 0.1266 & 0.1184 \\
\hline v4 & 0.0929 & 0.1266 & 0.1642 & 0.1537 \\
\hline v5 & 0.0876 & 0.1184 & 0.1537 & 0.1587 \\
\hline
\end{tabular}

\begin{tabular}{|c|c|c|c|c|}
\hline CS- & v2 & v3 & v4 & v5 \\
\hline v2 & 0.4067 & 0.4177 & 0.3949 & 0.2771 \\
\hline v3 & 0.4177 & 0.5992 & 0.5223 & 0.4843 \\
\hline v4 & 0.3949 & 0.5223 & 0.6310 & 0.4950 \\
\hline v5 & 0.2771 & 0.4843 & 0.4950 & 0.5114 \\
\hline
\end{tabular}

\begin{tabular}{|c|c|c|c|c|}
\hline Ctrl & $v 2$ & v3 & $\gamma 4$ & v5 \\
\hline$v^{2}$ & 0.5893 & 0.5367 & 0.5542 & 0.4053 \\
\hline$\gamma 3$ & 0.5367 & 0.5508 & 0.4989 & 0.3785 \\
\hline$v 4$ & 0.5542 & 0.4989 & 0.6408 & 0.3919 \\
\hline$\gamma^{5}$ & 0.4053 & 0.3785 & 0.3919 & 0.3657 \\
\hline
\end{tabular}

\section{6 $\mathrm{Y}$-KC Boutons Form Clusters That Are Changed in the Course of Associative Training}

In order to further characterize the diverse responses of the 1457 boutons of the 20 measured single $\mathrm{\gamma}$-KCs and determine if synchrony and asynchrony derives from grouping of boutons, an agglomerative hierarchical clustering (AHC) was applied. The AHC revealed that the boutons can be categorized into four different naïve bouton response classes (BRC, Fig. 3.15 a, dendrogram) based on their amplitudes and peak time point (Fig. 3.15 b) as the cost rose rapidly after merging 
cluster 3 and 4 (Fig. 3.15 a, right, derivative of costs). M ost of the boutons fell into BRC4 (53.98 \%) showing an early but comparably low response (Fig. 12 b, turquoise). The second group (20.57 \%) showed lower amplitudes with less sharp peaks (Fig. 3.15 b, BRC3 - dark yellow). The next group (20.78 \%) showed medium-high amplitudes with less sharp peaks (Fig. 3.15 b, BRC2 - purple), and the smallest group of boutons (4.67 \%) showed high amplitudes with comparably long latencies (Fig. 3.15 b, BRC1 - orange). In order to rule out that a cluster, especially the small cluster BRC1, is produced by a single $\mathrm{\gamma}-\mathrm{KC}$ showing this exact response profile, the amount of $\mathrm{\gamma}-\mathrm{KC}$ responses participating in a cluster were revised. $\mathrm{BRC1}$, despite being the smallest, was formed by 6 out of $24 \gamma$-KC responses. In the other BRC the following numbers of $\gamma$-KCs participated: BRC2 - 14, BRC3 - 19, BRC4 - 24. To investigate how olfactory associative training affects the diverse bouton activities, each bouton response of the three post training conditions (CS+, CS- and control) was assigned to one of the four BRCs they are closest to (Voronoi cells, Fig. $3.15 \mathrm{c}-\mathrm{f}$ ). The pre training condition with the naïve bouton responses formed the four clusters with their respective centroids (Fig. 3.15 c, black circles). The borders of each cluster were determined by forming the Voronoi cells around these centroids. How the post training conditions changed is shown and quantified in Fig. 3.16. Here, the fractions of bouton responses, classified to one of the BRCs, are displayed for each $\gamma$-lobe compartment (Fig. 3.16 a, color coded matrices and Tab. 3.4). Before the training, most of the boutons fell in BRC4 where most of those came from $\gamma 4$ followed by $\gamma 2, \gamma 3$, and least amount from $\gamma 5$ (Fig. 3.16 a, pre training). The least boutons fell into $\mathrm{BRC} 1$, the rest distributed between BRC2 and BRC3. All BRCs were formed by boutons from all $\mathrm{y}$-lobe compartments. After the training (Fig. 3.16 a, post training), the distribution of boutons changed in the CS+condition. Here, the amount of boutons decreased in BRC4 and increased in BRC1 and BRC2. In the CS- condition the opposite effect was observed. The amount of boutons decreased in BRC2 but slightly increased in BRC3 and BRC4. In the control, condition another effect was detected: no bouton, except for 1, was falling into BRC1 anymore. Furthermore, the number of boutons decreased in BRC2 and BRC3 but increased in BRC4. The changes of bouton distributions are quantified in Fig. 3.16 b. These results demonstrate that the naïve-formed BRCs were rearranged after the training depending on the training condition. This rearrangement led to a significant difference between training conditions in BRC1 (all groups were highly significantly different from each other). However, in BRC2 and BRC4 it led to equalizing of the CS- and control condition, whereas the CS+ condition was in both cases highly significantly different to them. In BRC3 all three conditions became more equal to each other due to the rearrangement. 

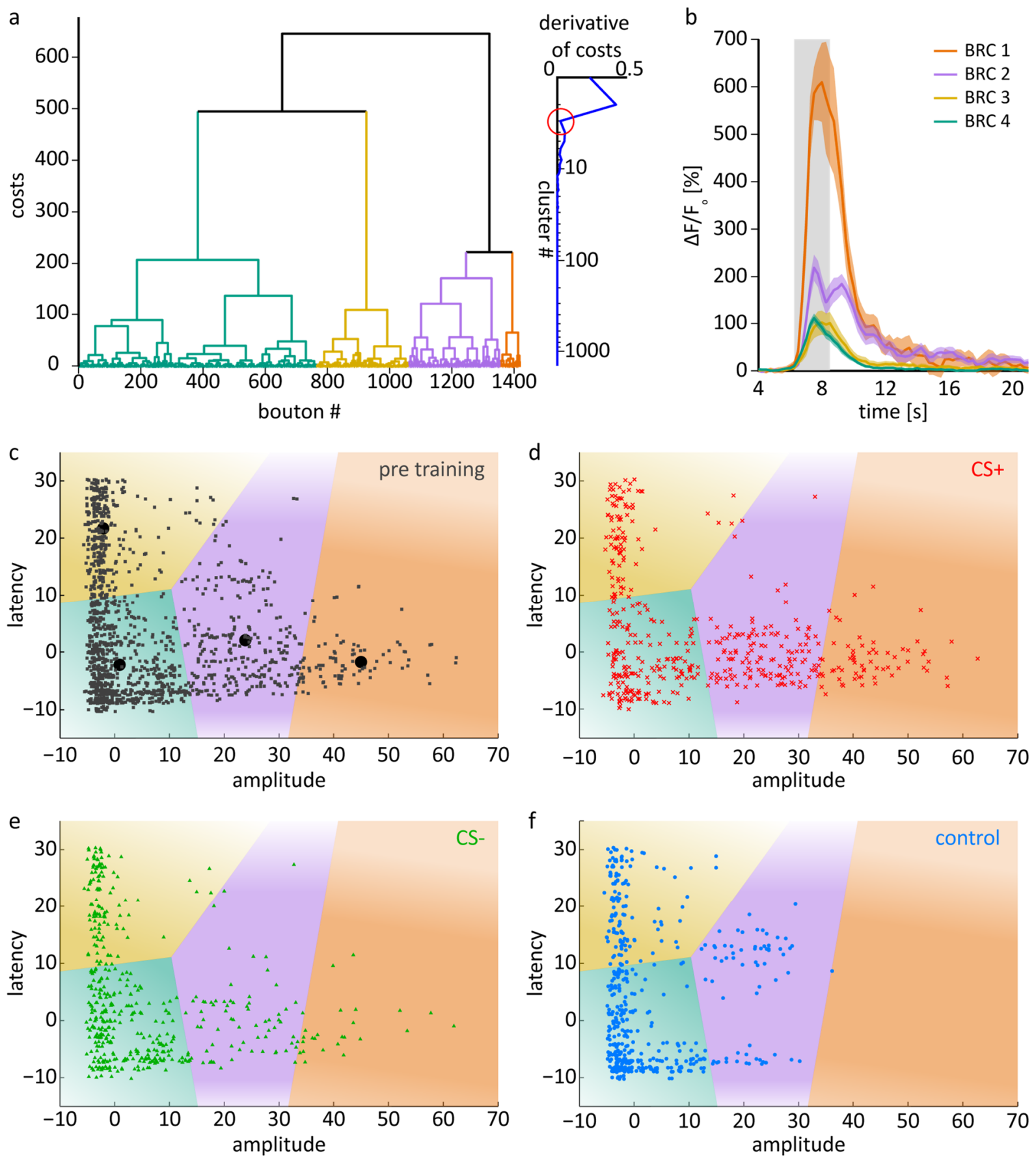

BRC 1

BRC 2

BRC 3

BRC 4

Figure 3.15: Agglomerative hierarchical clustering (AHC) of 1457 measured boutons with the amplitude and amplitude latency as feature space. a The pre training condition was used to cluster the 1434 bouton responses. The dendrogram shows the results of the AHC (left) showing the cost (Ward's criterion), forming four distinct clusters (called BRCs) indicated in different colors. Cluster number of 4 was determined by the derivative of the cost at which point the cost of merging two clusters rose rapidly (right, red circle). $\mathbf{b}$ Centroid response profile of each BRC shown as median traces and their $95 \% \mathrm{Cl}$ color coded based on a. Grey shade indicates odor stimulation window. c-f Voronoi cells based on pre-training-clustering color coded as in a showing the bouton distribution of the training conditions (pre - c, CS+ - d, CS- - e and control - f). Centroids of each pre-cluster are indicated as black circles in $\mathbf{c}$. Both data features were scaled to the respective standard deviation. 
Even though the boutons clustered in the BRCs (being inside a Voronoi cell), these clusters might change in their coherence due to associative olfactory learning. To test this, the variances within BRC clusters were calculated by measuring the Euclidean distances within BRCs (see Methods 2.2.8, Fig. $3.16 \mathrm{c}$ ). This analysis showed that the internal BRC variances were significantly higher in the CS+ and CS- condition than compared to the control, making the clusters within the BRCS more indistinct and variable.

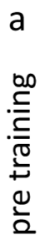
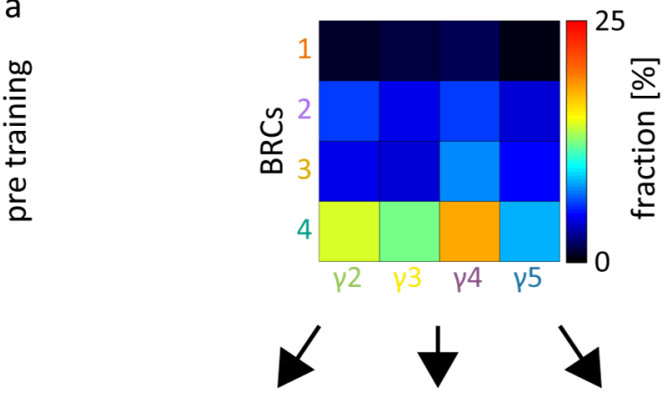

CS

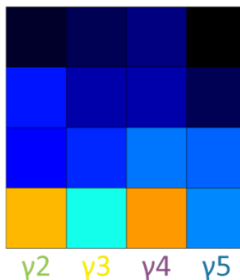

control
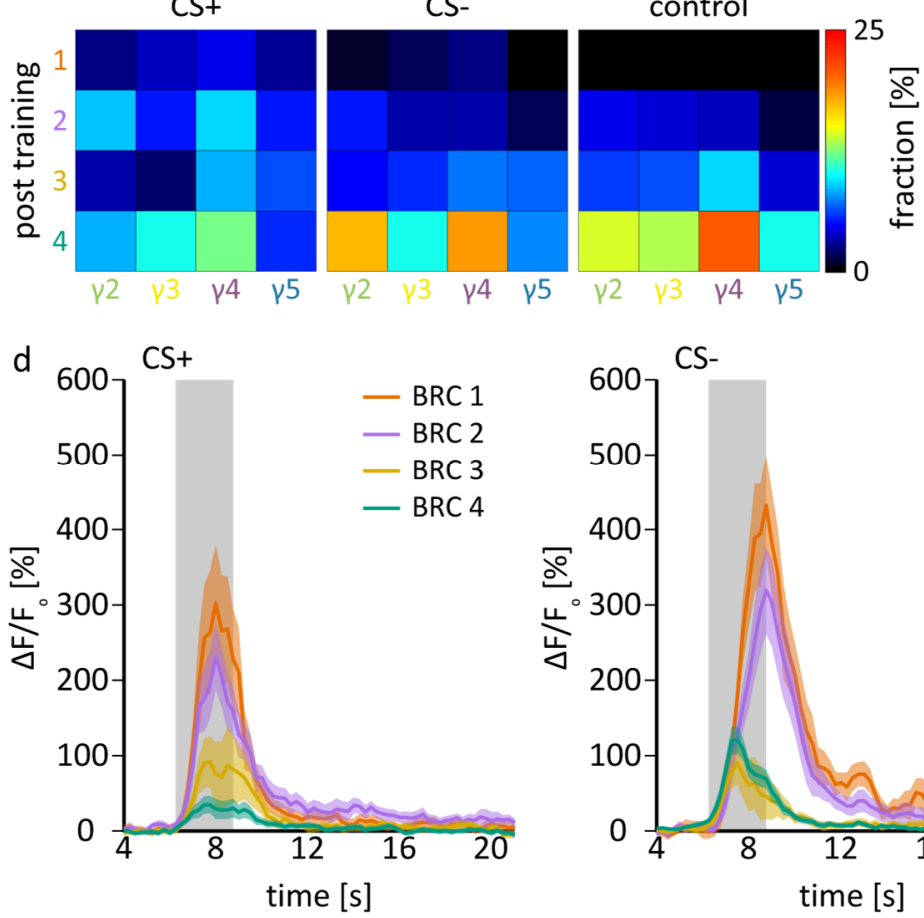

CS-



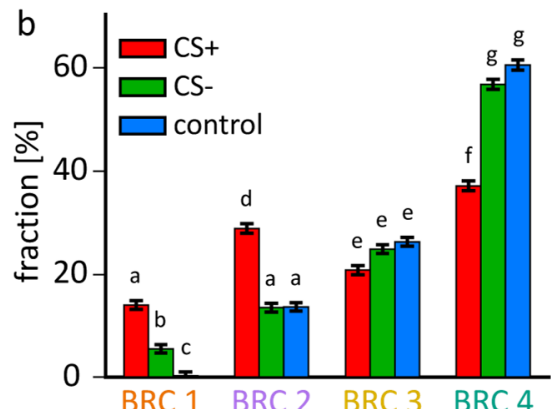

C
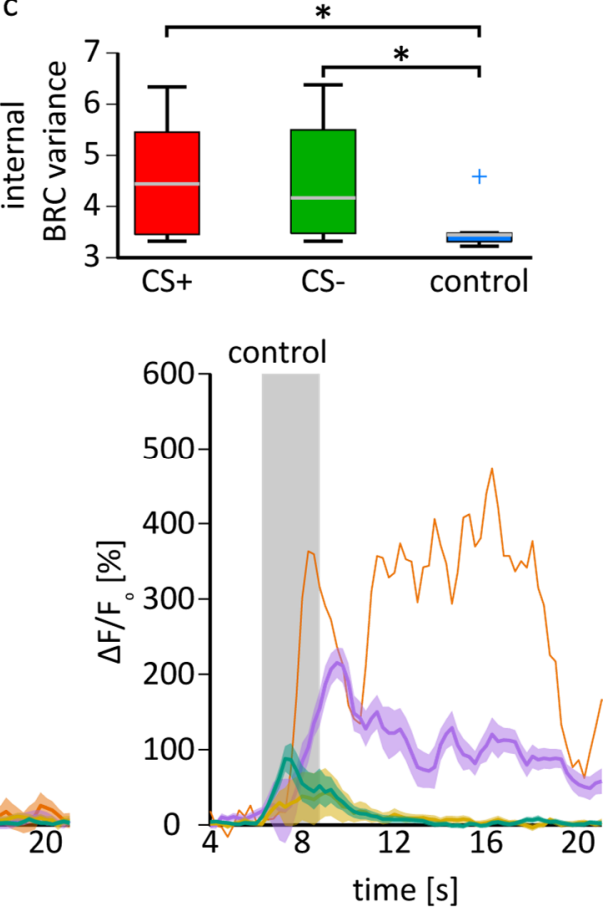

Figure 3.16: Shift of bouton distribution in the BRCs in the course of olfactory associative learning. a BRC fractions of boutons for each $y$-lobe compartment before and after the training (values in Tab. 3.5). $\mathbf{b}$ Quantification of bouton fractions of a for the CS+ (red), CS- (green), and control (blue) condition. Each bar indicates the median sum across $\psi$-lobe compartments (error bars are $95 \% \mathrm{Cl}$ ). As numerous comparisons were tested and significances couldn't be displayed anymore, letters were used to indicate significances. Bars with identical letters above them are not significantly different. All bars with different letters are strongly significant $\left(* * *, p<0.001 ; X^{2}\right.$ - test, corrected with Benjamini Hochberg FDR correction), with exception of a vs. $b, b$ vs. $d$ and $d$ vs. e which are ** significant $(p<0.01)$. c Overall internal BRC variance (sums of mean Euclidean distances matrix) after the training plotted as boxplots ( $25-75 \%$ quartiles). Grey lines indicate median, whiskers $1.5 \mathrm{x}$ interquartile distances. In the CS+ and CS- condition the internal BRC variance was significantly $(*, p<0.05$; Fisher's permutation test on median differences, corrected with Benjamini Hochberg FDR correction) higher than in the control condition. Blue cross indicates outlier. d Median traces (as in Fig. 3.15 b) of the clusters formed after the training within the respective BRCs for the 
training conditions CS+(left), CS- (middle), and control (right). In the control only one bouton fell into BRC1, therefore only the single trace of this bouton is shown.

The previous analyses showed a training specific change in the distribution of boutons in BRCs and an increased variance within BRCs for the CS+ and CS-. But how do the clusters change? To get an idea of how a BRC response looked after the training the median response dynamics of the BRCs were plotted in Fig. 3.16 d. In the CS+ and CS- condition, the median amplitudes of BRC1 decreased compared to before the training. Additionally, the BRC2 response increased in the CScondition so that in both cases BRC1 and BRC2 were converging. In the CS+condition, the median BRC4 responses were decreased in amplitude and showed a flattened plateau-like shape so that BRC3 and BRC4 diverged from each other. The opposite was true for the CS- condition, in which BRC3 changed to converge with BRC4. As already seen in the fractions analysis, the number of boutons drastically decreased in $\mathrm{BRC} 1$ for the control. There was only a single bouton that just fell (right at the border of the Voronoi cell, see Fig. 3.15 f) into $\mathrm{BRC1}$, therefore having only a single calcium trace. BRC2 showed a strong shift in amplitude latency and signal decay, whereas BRC3 decreased its response, converging with BRC4. These findings illustrate the reshaping of bouton odor responses due to aversive olfactory associative training.

Tables 3.5: Bouton fractions plotted in Fig. 3.16 a

\begin{tabular}{|c|c|c|c|c|}
\hline pre & v2 & v3 & v4 & v5 \\
\hline $\mathbf{1}$ & 0.9066 & 1.2552 & 1.7434 & 0.7671 \\
\hline $\mathbf{2}$ & 6.0669 & 4.6722 & 6.0669 & 3.9749 \\
\hline $\mathbf{3}$ & 4.3236 & 4.0446 & 7.4616 & 4.7420 \\
\hline $\mathbf{4}$ & 14.4350 & 12.1340 & 19.1770 & 8.2287 \\
\hline
\end{tabular}

\begin{tabular}{|c|c|c|c|c|}
\hline CS+ & $\boldsymbol{\gamma 2}$ & $\boldsymbol{\gamma 3}$ & $\boldsymbol{\gamma 4}$ & $\boldsymbol{\gamma} \mathbf{5}$ \\
\hline $\mathbf{1}$ & 2.6030 & 3.6876 & 4.5553 & 3.0369 \\
\hline $\mathbf{2}$ & 8.6768 & 5.2061 & 9.3275 & 5.4230 \\
\hline $\mathbf{3}$ & 3.4707 & 2.1692 & 8.4599 & 6.5076 \\
\hline $\mathbf{4}$ & 8.2430 & 10.4120 & 12.3640 & 5.8568 \\
\hline
\end{tabular}

\begin{tabular}{|c|c|c|c|c|}
\hline CS- & v2 & v3 & v4 & v5 \\
\hline $\mathbf{1}$ & 1.0460 & 1.6736 & 2.5105 & 0.2092 \\
\hline $\mathbf{2}$ & 5.2301 & 3.3473 & 3.1381 & 1.6736 \\
\hline $\mathbf{3}$ & 4.8117 & 5.6485 & 7.3222 & 6.9038 \\
\hline $\mathbf{4}$ & 18.4100 & 10.2510 & 20.2930 & 7.5314 \\
\hline
\end{tabular}

\begin{tabular}{|c|c|c|c|c|}
\hline Ctrl & $\boldsymbol{\gamma 2}$ & $\boldsymbol{\gamma 3}$ & $\boldsymbol{\gamma 4}$ & $\boldsymbol{\gamma 5}$ \\
\hline $\mathbf{1}$ & 0 & 0 & 0.2020 & 0 \\
\hline $\mathbf{2}$ & 4.4444 & 4.0404 & 3.6364 & 1.4141 \\
\hline $\mathbf{3}$ & 6.0606 & 6.4646 & 9.2929 & 4.2424 \\
\hline $\mathbf{4}$ & 14.1410 & 13.3330 & 22.2220 & 10.5050 \\
\hline
\end{tabular}

In order to quantify the BRC dispersion across the $y$-lobe compartments, Shannon's information entropy (Shannon, 1948) was calculated, measuring how many bits are needed to encode the combination of BRCS in each $y$-lobe compartment. Specifically, it was asked how associative training redistributes the $B R C$ representation potentially changing the input to $M B O N$ s. Figure 3.17 illustrates the median information entropy calculated for each $\mathrm{p}$-lobe compartment. Before 80 
the training, the four $\gamma$-lobe compartments showed a similar level of entropy, with $\gamma 2$ having the lowest in contrast to $\gamma 5$ having the highest entropy (see Tab. 3.6). This changed in the course of associative olfactory learning. In the CS+ condition the entropy increased in all $y$-lobe compartments, with $\gamma 4$ and $\gamma 5$ showing the highest entropy. In the CS- condition there was a slight decrease in entropy, except for $\gamma 3$, which increased. In the control condition a strong decrease in entropy was detected, being strongest in $\gamma 5$. These results are quantified across $\gamma$ lobe compartments in Fig. 3.17 b, which clearly demonstrates the change in information entropy and therefore the change in the BRC dispersion after the training. All groups were highly significantly different from each other, except the CS- condition which was indistinguishable from the pre-condition. In the CS+ condition the entropy increased indicating a more versatile BRC dispersion whereas in the control the entropy decreased, indicating a less variable BRC distribution. This data is in line with the previous similarity analysis (Fig. 3.14 b) in which the CS+ underwent strong de-synchronization. In contrast, in the control condition, the BRC dispersion became more invariable which is in line with the similarity analysis where the grouping of $\gamma^{2}-\gamma^{4}$ was sharpened after the training.

a

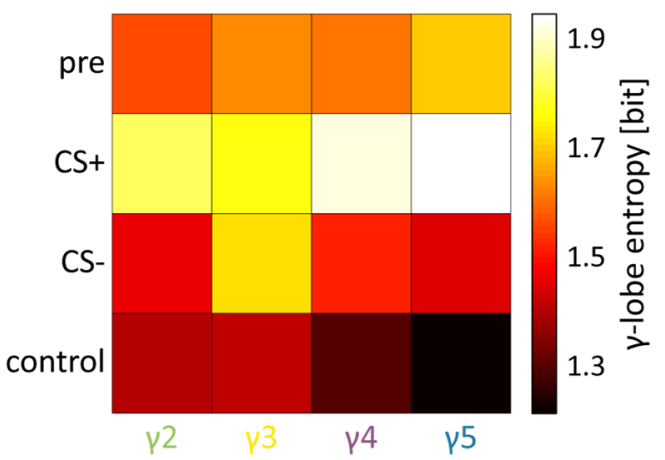

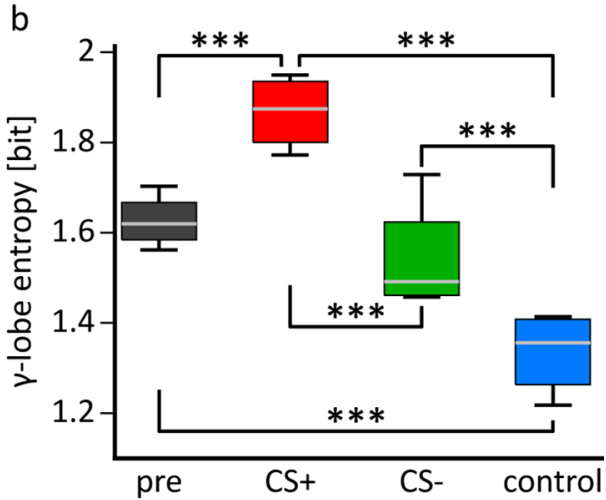

Figure 3.17: BRC dispersion changes in the course of olfactory associative learning. a Shannon's information entropy for each $y$-lobe compartment plotted as color coded matrix (values in Tab. 3.6). b Quantification of a across $\gamma$-lobe compartments in the respective training conditions before (pre, dark grey) and after the training (CS+(red), CS- (green) and control (blue)). Boxplots indicate 25 - $75 \%$ quartiles. Grey lines indicate median, whiskers $1.5 \mathrm{x}$ interquartile distances. All boxes are highly significantly different from each other $(* * *, p<0.001$, Fisher's permutation test on median differences, corrected with Benjamini Hochberg FDR correction) except pre and CS-.

Table 3.6: Shannon's entropy for the $y$-lobe compartments plotted in Fig. 3.17 a

\begin{tabular}{|c|c|c|c|c|}
\hline & v2 & p3 & v4 & p5 \\
\hline pre & 1.5617 & 1.6322 & 1.6075 & 1.7027 \\
\hline CS+ & 1.8287 & 1.7726 & 1.9208 & 1.9497 \\
\hline CS- & 1.4645 & 1.7288 & 1.5183 & 1.4574 \\
\hline ctrl & 1.4032 & 1.4134 & 1.3078 & 1.2180 \\
\hline
\end{tabular}


Results

In summary, $y$-KCs show highly individual odor response profiles with functional subdivision along the axons of the cells. Aversive olfactory associative training induces a de-synchronization of boutons when paired with an electric shock. Furthermore, bouton responses can be grouped into four response classes that are rearranged after the training for all training conditions. In addition, the information content is reduced in the CS+ condition, unchanged in the CS- condition, and increased in the control condition. 


\section{Discussion}

It is fascinating to learn how external stimuli are integrated, associated, and memorized in the brain to adapt the behavioral output appropriately. On this basis it is of high importance to understand how learning and memory is accomplished in neuronal networks that communicate by electrical and chemical information flow in large (e.g., mammalian) as well as small (e.g., insect) brains. Moreover, science has shown that learning and memory is not restricted to humans or other, "higher developed" animals. For example, honeybees - having a very small brain - perform well in complex forms of learning (M enzel, 2012; M enzel and Müller, 1996). That an even smaller insect like Drosophila is capable of performing in a variety of learning tasks is remarkable on its own (Kirkhart and Scott, 2015; Perisse et al., 2013a; Wolf et al., 1998).

As already introduced in the beginning, Drosophila is a well-suited organism to investigate the neuronal mechanisms of olfactory learning and memory. Here, the MB circuit, with its $\approx 2000$ intrinsic KCs per hemisphere (Aso et al., 2009), was shown to be the key structure involved in these processes (see reviews: Hige, 2018; Kaun and Rothenfluh, 2017; Owald and Waddell, 2015). Memories can be divided into different phases based on their retention times (see reviews: Davis, 2011; Heisenberg, 2003) and are formed based on differential processes and neuronal locations (see review: Busto et al., 2010; Davis, 2011; Owald and Waddell, 2015). Here, I show first, that $\gamma^{-}$ KCs are responding sparsely to odor stimulations and are functionally compartmentalized. Second, a short term memory trace located in $\mathrm{\gamma}$-KCs that is expressed in the form of synaptic desynchronization.

\subsection{Odor Coding in the $\mathrm{y}$-Lobe KCs}

The MB intrinsic KCs can be divided into three main classes and further into seven subclasses based on their anatomy, intrinsic protein and gene expression, birth order, and eventually their behavioral roles (Aso et al., 2009; Crittenden et al., 1998; Krashes et al., 2007; Lee et al., 1999; Perisse et al., 2013b, 2013a; Tanaka et al., 2008; Yang et al., 1995). Thereby, each M B-lobe has not just one segregated function but diverse ones depending on lobe type, and these lobes can also interact. The $\mathrm{y}$-lobe KCs were shown to be involved in the formation and retrieval of aversive and appetitive olfactory associative STM, M TM , and LTM (Akalal et al., 2006, 2010; Blum et al., 2009; Boto et al., 2014; Cervantes-Sandoval et al., 2013; Knapek et al., 2013; Scheunemann et al., 2012; Xie et al., 2013; Zars et al., 2000). Their olfactory input comes from PNs semi-randomly connecting 
to the calyx region of the MB (Butcher et al., 2012; Caron et al., 2013; Gruntman and Turner, 2013; Tanaka et al., 2004; Wong et al., 2002; Yasuyama et al., 2002). Each odor activates only a sparse weakly-overlapping set $(\approx 5 \%$ ) of KCs (Honegger et al., 2011; M urthy et al., 2008; Turner et al., 2008).

In a first step of this study it was investigated how odors are coded in the lobe region of $y$-KCs. Studies investigating odor coding so far always looked at the cell soma layer or calyx. However, the question remains if the activity in these layers represents the activity of the axonal regions. It was shown that several of the $\approx 7$ claws per $\mathrm{KC}$ must receive input to elicit spiking measured in the soma (Butcher et al., 2012; Gruntman and Turner, 2013; Lee et al., 1999; Leiss et al., 2009). Is this soma-spiking sufficient to propagate down to the axons in the lobes? This is important to know as the axonal outputs of KCs are the key features in the learning processes. Even though calcium imaging in the cell soma layer shows a convenient possibility to investigate single $\mathrm{KC}$ responses (Honegger et al., 2011), the KC soma layer lacks the information of KC type identity. It might well be that different $K C$ types $\left(\gamma, \alpha^{\prime} / \beta^{\prime}\right.$, and $\left.\alpha / \beta\right)$ have different response probabilities. This was indicated by electrophysiological studies filling single $\mathrm{KCS}$ after patch-clamp recordings to investigate the KC type identity (Turner et al., 2008). They found that the response probabilities were lowest for $\gamma$-KCs, higher in $\alpha / \beta-K C S$, and highest in $\alpha^{\prime} / \beta^{\prime}-K C s$. To investigate the responsiveness of the $M B$-lobes, calcium imaging would be an appropriate tool to visualize odor responses but this completely lacks single cell resolution. To overcome this problem I used the MARCM technique (Lee and Luo, 1999) to induce GCaMP3 (Tian et al., 2009) expression in single cell clones restricted to $\mathrm{y}$-KCs by using 5HT1B-GAL4 (Yuan et al., 2005). I found that 50 of 262 measured $y$-KCS reliably responded to one or a combination of several odors tested in this study and the solvent M Oil. M CH and 3-Oct elicited responses in $\approx 5 \%$ of the cells - in line with previous studies (Honegger et al., 2011; Turner et al., 2008). In contrast, 1-Oct and M Oil only elicited responses in $\approx 1 \%$ of $y$-KCs (see 3.3 and Tab. 3.1). These differences can be due to odor identity, also found by others (Honegger et al., 2011). Interestingly, the response probability to two or more odors were $\approx 1 \%$, and in most cases even below, showing the impressive non-overlap in KCs. The observed small overlap of rather dissimilar odors e.g., MCH and 3-Oct was also seen in another study where these two odors elicited responses in $\approx 30 \%$ of the KCs responding to one of them (Hige et al., 2015a). These results show that odor responses are indeed sparse in the lobes, as well with a low level of overlap, keeping the odor code distinct. This sparse coding gives the opportunity to the system to encode a lot of information in a given set of cells forming a large coding space and is relatively energy saving. The low overlap between odor representations minimizes the synaptic interference as only small sets of cells respond to the same stimulus (Hige et al., 2015a; Olshausen and Field, 2004; Spanne and Jörntell, 2015). 


\subsection{B-Extrinsic Innervations Tile $y-K C$ Axons Functionally}

MB extrinsic DANs and MBONs tile the KCs into stereotypic, highly overlapping compartments each of which could be shown to have specific functions and roles in learning and memory (Aso et al., 2014b; Kaun and Rothenfluh, 2017; Owald and Waddell, 2015; Perisse et al., 2013a). But does this compartmentalization also affect the physiologic properties of a single KC? Specifically, do branches of a $\mathrm{KC}$ residing in one compartment respond differently to odors when compared to branches residing in another compartment of the same cell? Can $\mathrm{KC}$ axons be also subdivided into functional units?

Investigation of single $\mathrm{y}$-KCs in this study has shown that the axons comprise bouton-like structures, which show immunoreactivity to the pre-synaptic protein BRP localized to mainly the boutons (see 3.1.2 and Fig. 3.3). Therefore, it is highly likely that these boutons are the synaptic sites at which DANs and MBONs are connected to the $\mathrm{\gamma}$-KCs and plastic changes in the course of learning occur. The boutons could be assigned to the $\gamma$-lobe compartments they are part of and therefore analyzed compartment-specifically. Comparing the bouton responses in single $\mathrm{y}$-KCs showed an unexpected, though remarkable, individuality in that they did not uniformly respond along the whole axon. Instead, some compartments showed higher numbers of responsive boutons than others, ranging from only one active up to all active compartments (see 3.4.1 and corresponding figures). This effect is in line with other studies (Barth et al., 2014; Caron et al., 2013; M urthy et al., 2008), though never shown on a single cell level. In order to investigate if the extrinsic compartmentalization can also be found to be functionally intrinsic to $\gamma-\mathrm{KCs}$, I, in collaboration with Dr. Bart Geurten, developed a similarity measurement that compared the similarity of boutons within a compartment to boutons of other compartments, taking into account the cross-correlation between boutons and their response intensities (see 2.2.7). This analysis revealed that the boutons of $\gamma$-lobe compartment 2-4 are significantly more similar within their compartments, indicating a functional segregation of single KCs (see 3.4.2 and Fig. 3.10). These results indicate that the odor coding space does not simply arise from the number of KCs that are activated upon odor stimulation (Honegger et al., 2011; Luo et al., 2010; M urthy et al., 2008; Turner et al., 2008) but has to be extended by the number of compartments, implicating a massive expansion of the odor coding space. As the $\gamma 1$ compartment was not unambiguously analyzable it couldn't be investigated; however, this region might potentially be an additional functional unit further extending the odor coding space. Although the response probability of $y$ - 
KCs remains the same in the lobes compared to the cell somata, these findings show the importance of investigating odor responses at the level of the lobes.

How can this functional segregation be mediated? One possibility is that local circuits between KCS, DANs, and MBONs at each bouton site regulate voltage gated calcium channels giving rise to differential calcium influx upon odor stimulation. This possibility is supported by the recent connectome studies of a larva (Eichler et al., 2017) as well as the $\alpha$-lobe of an adult MB (Takemura et al., 2017). These impressive studies revealed two new circuit motifs that are KCs synapsing onto DANs and DANs synapsing onto MBONs. These reciprocal synapses might modulate bouton responsiveness locally. As DANs and MBONs are innervating the KCs compartment specifically, this modulation might be stronger within a compartment than in between. Further evidence comes from a study in which the artificial activation of MBONs leads to the activation of DANs distal to this MBON possibly regulating feedforward loops in MB lobes (Cohn et al., 2015). Additionally, the mutation or downregulation of DA-receptors in KCs led to reshaping of the odor response pattern in the $\mathrm{y}$-lobe (Cohn et al., 2015). Furthermore, KCs are interconnected via chemical synapses (Takemura et al., 2017) and gap-junctions (Liu et al., 2016), which can be further modulated by hormones or neurotransmitters (Marder et al., 2017), adding another level of complexity to the circuit. Although the connectome studies reconstructed so far only the adult $\alpha$-lobe and the larval $y$-lobe, a similar connectivity motif might be true for the adult $y$-lobe. A second possible mechanism for forming functional units in $\gamma$-KCs is the APL circuit that was recently shown to be not just a global inhibitor for the odor input in the calyx (Lin et al., 2014a; Liu and Davis, 2009) but also acts locally at specific KC types (Inada et al., 2017). Furthermore, the APL neuron was shown to be multipolar in terms of dendritic and axonal regions also providing input to MB lobes, including $\mathrm{y}$-KCS (Wu et al., 2013). These features provide a further circuit modulation that might lead to a local as well as compartment-specific odor responsiveness of synaptic sites (boutons). A third possible mechanism was indicated by the manipulation of the $G_{0}$ protein in $\mathrm{y}$-KCs with pertussis toxin (PTX), which influenced the odor responsiveness of $\mathrm{y}$-KCS (Zhang and Roman, 2013). The $G_{0}$ activity might be locally regulated, shaping odor-induced activity in single boutons. In vertebrates the $G_{0}$ protein and $G_{\beta \nu}$ protein sub-unit modulate voltage gated calcium channels (Hescheler et al., 1987; Ikeda, 1996) a mechanism that might also shape calcium-mediated odor responses in Drosophila $\gamma$-KCs. The above described mechanisms are possible explanations for the functional subdivision within $\mathrm{v}$-KCs, but it is likely that it is a combination of all, indicating the immense complexity of the MB circuit. 


\subsection{Aversive Associative Learning Changes the Reinforced Odor Representation at the Synaptic Level in $\mathrm{\gamma}$-KCS}

It was shown in many studies that the CAMP-pathway is the key player in the coincidence detection of CS and US in the MB and especially important in $\mathrm{\gamma}$-KCS (see review: Kahsai and Zars, 2011). In classical conditioning experiments it could be shown that different DAN clusters convey the aversive (PPL1) and appetitive (PAM) stimuli to KCs and if odor-activated calcium influx in KCS precedes DA activated G-protein activation, the US is associated with the CS (see review: Kaun and Rothenfluh, 2017). Electric shocks used as aversive US in olfactory associative conditioning activate DANs innervating the $p$-lobe compartments 1-3 (Aso et al., 2010; Cohn et al., 2015; Riemensperger et al., 2005) whereas sugar presentation used as appetitive US activates DANs innervating the $y$-lobe compartments 4 and 5 (Cohn et al., 2015). This innervation pattern tiles the $\gamma$-lobe in two halves presumably promoting aversive and appetitive memory. MBONs were shown to promote avoidance or approach after associative conditioning in a compartment-specific way (Aso et al., 2014b and review: Owald and Waddell, 2015). Interestingly, $\mathrm{y}$-lobe MBONs that are connected to DANs conveying punishment drive the contrary approach behavior and vice versa, indicating that associative learning might induce depression at those KC-MBON synapses (e.g., ${ }^{1}$ or $\gamma 2$ ) and potentially synaptic facilitation at the opposing synapses (e.g., $\gamma 4$ or $\gamma 5$ ). Indeed, studies using calcium imaging found that the CS+ response in $\gamma 1$ ped-MBON driving approach is depressed (Perisse et al., 2016) and the CS+ response in $\gamma 5 \beta^{\prime} 2 a-M B O N$ driving avoidance is increased (Bouzaiane et al., 2015) after aversive conditioning. Furthermore, a study in our lab using synaptically-tagged GCaMP (Pech et al., 2015) expression in MBONs shows a depression in the $\gamma 1$ ped-MBON and an increase in the $\mathrm{Y} 4-\mathrm{MBON}$ CS+ response $3 \mathrm{~min}$ after aversive conditioning indicating the STM component in the $\gamma$-lobe associated circuit (Clare Hancock, personal communication). How does this change in M BON activation after associative training come about? What happens after associative training to the $\mathrm{y}$-KCs?

In order to investigate the associative training-induced changes in $\mathrm{y}-\mathrm{KCS}$, flies expressing GCaMP in single $\mathrm{\gamma}$-KCs were subjected to a differential aversive training protocol under the microscope (see 2.2.3). The single $\mathrm{KC}$ calcium imaging had the great advantage that associative traininginduced changes could be investigated at the synaptic level (boutons) in respect to the compartmentalization. Calcium imaging studies in the MB so far always lacked the single cell and further single synapse resolution (Akalal et al., 2010; Boto et al., 2014; Wang et al., 2008). Furthermore, training under the microscope enables the investigation of a STM trace of a few minutes after training, which is usually successfully tested in behavioral paradigms (Barth et al., 2014; Tully and Quinn, 1985). In other studies, this investigation was not possible as they trained 
flies in conditioning apparatuses (Tully and Quinn, 1985), subjecting the flies to further stress because of handling, anesthetizing, and imaging preparation procedures (e.g., Akalal et al., 2010; Wang et al., 2008). Analyzing the changes in calcium responses compartment-wise, each $\mathrm{Y}-\mathrm{KC}$ showed individual changes, either increasing or decreasing odor responses, after the associative training when compared to their naïve response (see 3.5 and Fig. 3.11). One fly which had expression in two $\mathrm{Y}$-KCS - one responding to the CS+ and the other to the CS- - showed highly interesting training effects: whereas the responses to the CS+ decreased mainly uniformly across compartments in one cell, the CS- recruited bouton responses in $\mathrm{p}$-lobe compartment 4 and 5 in the other cell. This example demonstrates a compartment-specific modulation of $\gamma$-KC responses within the same axon in the course of associative learning and antagonistic changes within the same fly. Looking at the data across flies, there was no general depression or facilitation in any training condition (CS+, CS- or control); however, a tendency could be detected in the CS+ pointing towards a decrease in calcium responses. Importantly, by applying the similarity analysis to the data before and after training we found a striking effect for the CS+, showing a significant de-synchronization of bouton responses within $\gamma$-lobe compartment 3-5 and between $\gamma$-lobe compartments (see 3.5 and Fig. 3.14 b). In the CS- condition a trend was observable that $\gamma$-lobe compartment 3 and 5 became more similar to each other. Strikingly, this CS- effect fitted complementarily to the CS+ effect, showing no change due to associative conditioning between $\mathrm{Y}$ lobe compartment 3 and 5. This is a possible site at which CS+ is further differentiated from CS-. These results indicate that there is a STM memory trace that can be found in $\mathrm{\gamma}-\mathrm{KCS}$. This memory trace is reflected in a de-synchronization of $\mathrm{y}-\mathrm{KC}$ bouton activity after pairing an odor to an electric shock. How can such compartment specific changes occur? As described in the previous section (4.2), the enormously complex circuit of feedforward, feedback excitation and inhibition is a possible candidate mechanism for not only modulation of naïve odor responses but also reshaping odor responses at single synaptic sites due to modulation of e.g., voltage gated calcium channels. Studies investigating the role of the $G_{0}$ protein in STM formation have shown that $G_{0}$ activity is necessary to form aversive and appetitive STM in $\gamma$-KCs, as well as in $\alpha / \beta$-KCs (Ferris et al., 2006; Madalan et al., 2012; Zhang and Roman, 2013). Besides the direct role of $G_{0}$ in associative learning, it further indicates that the same protein can mediate differential functions in the same neuron depending on localization, as different $y$-lobe compartments are involved in different types of learning.

So far there were only a few studies searching for memory traces in $\mathrm{\gamma}$-KCS, using calcium imaging, which obtained different results. Akalal and colleagues (2010) did not find any memory trace after single trial aversive conditioning in the $\gamma$-lobe, only after five times spaced training (regarded as LTM). Boto and colleagues (2014), artificially activating PPL1-DANs using dTrpA1, detected 
facilitation in the $y$-lobe 5 min after aversive artificial training as well as artificial appetitive training using dTrpA1 in PAM-DANs. Why are studies so different from each other? Results of scientific studies always have to be treated carefully and interpreted in respect to the setup used. Akalal et al. (2010) used GCaM P1.6 - an old version of GCaM P that might not have been sensitive enough to detect calcium changes after single trial conditioning and might have been under the detection threshold by the time they performed calcium imaging. Furthermore, flies were trained outside of the microscope and were therefore subjected to handling stress afterwards. Boto et al. (2014) used artificial activation of DANs to substitute the electric shock. This artificial activation might have led to stronger DA transmission than an electric shock. Furthermore, they did not differentiate the calcium signals by $v$-lobe compartments, which might have shown where this increase came from. These differences, together with the earlier mentioned compartmentspecific functions in learning and memory, emphasize the importance of compartmentsegregated analysis as performed in my study. Furthermore, it shows that the search for memory traces requires the analysis of a combination of parameters. A study performed in our lab (Barth et al., 2014) addressed a partially related question in which similar odors were differentially trained against each other. They could find that a STM memory trace after associative conditioning of similar odors under the microscope is not formed as direct change in calcium levels but could be detected in form of decorrelation of calcium representations. As they also used a whole lobe approach, they could not investigate the changes at a single cell or single synapse level but instead used an elegant pixel based analysis. This decorrelation of pixels underlines my findings that individual $\mathrm{y}$-KCs undergo differential changes in the course of associative learning and that on the population level no concrete changes could be detected. This also indicates that the information about the odor is kept within the MB, as a global depression of all responding $\gamma$-KCS would lead to a loss of odor information. Furthermore, de-synchronization can overcome the problem of synaptic interference (Hige et al., 2015a; Olshausen and Field, 2004; Spanne and Jörntell, 2015), keeping odor information constant but modulating synapses so that they can respond to both CS+ and CS- differentially. This allows for synapses to be assigned to different tasks depending on with which boutons they are correlated. As MBONs innervate the KCS only in their distinct compartments of the axons, it makes sense that synchrony-related plasticity is compartmentalized. This allows synapses of one compartment within a KC to desynchronize from a group of some other KCs and synchrony of synapses of another compartment to other groups of KCs. This plasticity mechanism functionally divides and groups synapses within single $\mathrm{KCS}$, respectively. In vertebrates, it was shown that the calcium-dependent intracellular GTPase (Ras) signaling cascade modulates neighboring synaptic spines in CA1 pyramidal hippocampus neurons (Harvey and Svoboda, 2007; Harvey et al., 2008). A similar mechanism 
could account for synchronization or de-synchronization in neighboring boutons of $\mathrm{\psi}$-KCs as Ras is also present in KCS, where it has important roles in axon growth (see review: Hall and Lalli, 2010) but likely also regulates other cellular functions like synaptic plasticity (Harvey et al., 2008). This modulation of synaptic plasticity can be locally distinct due to the compartment-specific circuitry (see 4.2).

But what does this de-synchronization mean for the KC output e.g., the MBONs? The dendritic tree of a MBON integrates signals from a large population of $\mathrm{KC}$, indicating a summation of dendritic inputs (Hige et al., 2015b; Tanaka et al., 2008 and see review: Stuart and Spruston, 2015). If the inputs to the MBON arrive asynchronously, potential post-synaptic currents might be insufficient to elicit spiking or may alter spike trains in the MBON - reflected in decreased calcium transients (Hige et al., 2015a; Perisse et al., 2016; Séjourné et al., 2011). This reduced odor response in MBONs compared to naïve odor presentation might alter the net drive of the MBON ensemble in a way that the animal will avoid this stimulus. A similar effect in which the timed and spatial integration of spikes alters the synaptic output can be found in the MBs of locusts (Cassenaer and Laurent, 2007) and hippocampus or cerebellum in vertebrates (see review: Roberts and Bell, 2002; Stuart and Spruston, 2015). It has to be noted that measurements in $M B O N s$ are done in the dendritic tree. The effect of dendritic integration in MBONs onto the output has not been investigated yet.

As we saw that boutons have the potential to act in synchrony, we asked whether certain response patterns lead to a functional grouping of boutons. Indeed, we found with agglomerative hierarchical clustering (see 2.2.8) that $\mathrm{p}$-lobe boutons can be divided into four bouton response classes (BRCs, see 3.6 and Fig. 3.15), which form compartment-specific naïve odor response patterns, or odor codes. Most strikingly, this odor code is significantly changed after associative training, separating the BRC pattern in the CS+ condition from the CS- and control (see 3.6 and Fig. 3.16). The fractions of boutons falling into the four BRCs in the CS- and control condition became more similar to each other but, interestingly, in the control condition one class was not formed anymore (except for one bouton). This rearrangement of the odor patterns indicates a change in the odor representation that likely changes the input to the downstream MBONs. In order to investigate how the odor representation is changed in and across $\psi$-lobe compartments, Shannon's information entropy (Shannon, 1948) was calculated. Information entropy is, in other words, the measure of uncertainty of the predictable outcome of an event or a measure for the reliability of a neural code (see review: Borst and Theunissen, 1999). Naïvely, the information entropy is somewhat equal across $y$-lobe compartments but lowest in $y$-lobe compartment 5 . This changed significantly after the training for the CS+ and the control condition but not the CS- (see 3.6 and Fig. 3.17). Interestingly, in the CS+condition, the entropy increased whereas the entropy 
in the control condition decreased significantly. Due to this phenomenon the odor code in the $\gamma^{-}$ lobe becomes more versatile, which might provide more uncertain input to the MBONs after pairing an odor with electric shocks (CS+). This uncertain input likely decreases drive to the MBONs, which in turn changes the net weighted output of the MBON ensemble, modifying the behavioral outcome. This is in line with the previous observation in our similarity analysis where a strong de-synchronization was detected. Both ways of analysis indicate that associative conditioning decreases certainty of the $y$-lobe output. This decrease in output could lead to the depression in MBONs seen in other studies after associative conditioning (e.g., Hige et al., 2015a; Perisse et al., 2016; Clare Hancock, personal communication). In the control condition, where no other stimulus than the odor was presented (no US), the odor code was less variable. This was also indicated in the similarity analysis where the odor response pattern in the control appeared to pronounce the naïve odor representation (see 3.5 and Fig. 3.14). This might strengthen the initial naïve behavioral output to this odor.

In conclusion, both ways of analysis showed that aversive olfactory associative learning changes the representation of odor information in the $\gamma$-lobe, likely affecting the output to MBONs and possibly DANs through reciprocal synapses (Eichler et al., 2017; Takemura et al., 2017). This change in MBON activity is likely changing the net ensemble output of MBONs that guide adequate behavioral responses.

\subsection{What Can Drosophila Tell Us About the Engram?}

The search for memory traces or the engram (Semon, 1904) in the brain is one of the key aspects in neurosciences investigating learning and memory. The engram is located to the brain in which specific neurons integrate external cues that cause plastic changes in these neurons to form a memory. External or internal triggers can retrieve this memory, eliciting behavioral outputs for which these neurons are sufficient and required (Gerber et al., 2004; Josselyn et al., 2015; Semon, 1904). By visualizing neuronal plasticity using calcium imaging, several engrams could be found in Drosophila residing mainly in the MB circuit (Davis, 2011). MB intrinsic KCs exhibit several forms of memory traces that depend on the KC type. STM traces were found in the $\mathrm{\gamma}$-lobe (Boto et al., 2014) and the $\alpha^{\prime} / \beta^{\prime}-$ lobe (Wang et al., 2008), both showing increased calcium responses for the

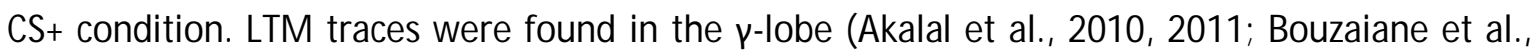
2015) and the $\alpha$-lobe (Yu et al., 2006) showing increased calcium responses to the CS+ presentation as well. However, memory traces are not only stored in KCs. They can be found in MB extrinsic neurons such as MBONs (Hige et al., 2015a; Owald et al., 2015; Pai et al., 2013; 
Perisse et al., 2016; Séjourné et al., 2011), the APL neuron (Liu and Davis, 2009), the DPM neuron (Yu et al., 2005), TH-GAL4 positive DANs (Riemensperger et al., 2005), and, interestingly, already at the level of PNs (Ashraf et al., 2006; Yu et al., 2004). Here I demonstrate another so far never shown form of calcium transient-dependent plasticity that is not explained by mere response intensities but the synchrony of single synaptic sites (boutons). As the above mentioned studies lack single cell and single synapse resolution, they might have missed memory traces. Even though insects are somewhat different from vertebrates, they share many common principles and anatomical similarities involved in learning and memory.

In vertebrates the cerebellum was shown to be the main brain structure involved in classical conditioning of reflexes (e.g., eye-blink conditioning; see review: Thompson and Steinmetz, 2009), showing remarkable similarities to the insect M B circuit (see e.g., Schürmann, 1974; Yasuyama et al., 2002). In brief: glutamatergic mossy fibers convey the CS information (conforming the PN input to MBs) to granule cells with its parallel fibers (conforming MB intrinsic KCs). Glutamatergic climbing fibers convey the US information (resembling dopaminergic PPL1/PAM input to the MB) to the cortical Purkinje cells (can be regarded as the MBONs) that are also connected to granule cells. Purkinje cells, as well as mossy and climbing fibers, project onto deep cerebellar nuclei (e.g., Interpositus) that are required for the CR expression (Schürmann, 1974; Thompson and Steinmetz, 2009; Yasuyama et al., 2002). Two main memory traces were found in the cerebellar circuit: one in the Interpositus nucleus showing increased responses to the CS presentation predicting the US and another one in Purkinje cells showing synaptic depression and facilitation. Both memory traces were found to work somehow independently of each other though the role of the Purkinje cell layer-related memory trace supposedly has more a modulatory role, acting on the Interpositus-related memory trace (see review: Thompson and Steinmetz, 2009).

Besides the cerebellum, other brain areas in vertebrates show memory traces in the course of classical conditioning, e.g., the amygdala in fear conditioning (see review: Maren, 2001). Here, the basolateral complex (BLA) was shown to be required for the acquisition and storage of fear conditioning, whereas the central amygdaloid nucleus is needed for the generation of conditioned fear responses (CR). Visual, auditory, as well as shock stimuli enter the amygdala through thalamic and cortical tracts conveying both the CS and the US. The coincidence of both alters synaptic plasticity in the form of increased responses in neurons of the BLA which show a short term component and a protein synthesis dependent long term potentiation that also involves voltagegated calcium channels (Maren, 2001). Together with the amygdala, the hippocampus is involved in fear conditioning. However, the hippocampus exhibits a more contextual conditioning component that is e.g., the behavioral set up in which the animal is trained. This means, that the placement of the animal into this set up can elicit the CR that is conditioned through the actual 
CS-US pairing (Holland and Bouton, 1999; Maren, 2001). The hippocampus is furthermore a key structure for place learning in which the so-called place cells play the major role and might be related to contextual learning as place cells can store information about location in a certain context (see review: Moser et al., 2015).

The importance of the dopaminergic system in classical conditioning in Drosophila - relaying aversive and appetitive valence - was already described previously (see also: Kaun and Rothenfluh, 2017). The tiling of the Drosophila brain by DAN clusters is a conserved feature that also exists in vertebrates and mainly involves the basal ganglia relaying punishment and reward in learning paradigms (Scaplen and Kaun, 2016). Even though insect (e.g., Drosophila) brain structures resemble those of vertebrates, the insect brain doesn't have this elaborated distribution of forebrain centers involved in different learning and memory tasks. In the insect brain, the MBs are the key structures that serve the many functions involved in learning and memory. This aspect of simplicity and the physiological similarities makes the investigation of learning and memory principles in Drosophila a suitable approach.

In this study, only $\mathrm{\gamma}-\mathrm{KC}$ activity was measured. To investigate what happens on the complete circuit level is a very challenging task as the MB circuit was recently shown to be enormously complex (Eichler et al., 2017; Takemura et al., 2017) and remains to be elucidated. However, this study contributes an important aspect into the field of synaptic plasticity in M B intrinsic KCs that was not shown so far. First, it shows that synaptic plasticity in the MB of Drosophila resides in the pre synapse, where the memory trace is embodied in form of the change in synaptic weights. Second, giving the findings that single spines in Purkinje cells of the vertebrate cerebellum can be activated by single parallel fibers of granule cells (Denk et al., 1995) and that localized, clustered as well as dispersed calcium influx is integrated over time and space in dendrites (Stuart and Spruston, 2015) indicates that an uncorrelated parallel fiber input reduces the response of the Purkinje cell. As parallel fibers resemble the MB KCs and Purkinje cells the MBONs, this mechanism of synchronized or de-synchronized plasticity is likely a shared mechanism. This kind of synaptic plasticity likely influences learning and memory performance in vertebrates and is now shown for the first time in the invertebrate MB. It furthermore fulfills one major criterion of defining a memory trace: synaptic plasticity within the neuronal substrate (Gerber et al., 2004; Martin et al., 2000; Thompson, 2005). Future experiments have to further elucidate the exact circuit mechanism for this kind of synaptic plasticity. 


\subsection{Outlook}

The work presented here provided new insights into a form of synaptic plasticity after aversive olfactory conditioning that is synaptic de-synchronization. Synaptic de-synchronization is the dissimilarity of synaptic activity between synaptic sites within the axon of a neuron with respect to temporal and amplitude aspects. With the techniques used here I was able to image at the single synaptic level and showed that aversive olfactory associative learning de-synchronized activity of synaptic sites in $\mathrm{Y}$-KCs. This was done using the cytosolic calcium sensor GCaM P3 (Tian et al., 2009) that shows the external and internal calcium influx of the cell indicating the activity and integration mechanisms within the axon. To localize calcium transients to the proximity of vesicle release or transmitter receptor sites, GCaMPs that are tagged to specific proteins of the pre-synaptic active zone (vesicle release, e.g., syp-GCaMP3) or the post-synaptic density (transmitter binding to receptors, e.g., homer-GCaM P3) can be used in future experiments (Pech et al., 2015).

As it was described that synaptic de-synchronization is a possible mechanism to reduce synaptic interference and overlap it would be highly interesting to understand how the MB deals with similar odors, especially in the light of the recent publication showing that similar odors can be trained to be differentiated in a differential training paradigm (Barth et al., 2014). Here, the technique using single KC imaging and the analysis of similarity of single synaptic sights can shed light on the mechanism of differentiation.

In order to uncover the mechanism that is leading to synaptic de-synchronization, a strategy for manipulating single cell intrinsic or extrinsic circuit function would be suitable. Using RNA interference (RNAi; Boutros et al., 2004) in combination with MARCM (Lee and Luo, 1999) downregulating the function of certain candidate enzymes of the cellular signaling cascade within a single KC can reveal possible cascade elements that mediate intrinsic modulation of synapses. In order to investigate the role of external mechanisms as indicated by the connectome studies (Eichler et al., 2017; Takemura et al., 2017), RNAi strategies knocking down DA receptors (dDA1, DAMB; Han et al., 1996; Kim et al., 2003) or innexins (Liu et al., 2016) to downregulate gapjunction function would give indications for possible extrinsic influences. Furthermore, the block of voltage gated $\mathrm{Ca}^{2+}$ channels via the application of certain spider toxins (PLTX and HoTX; Leung et al., 1989) can show the impact of calcium influx on synaptic plasticity mechanisms. It is likely that the output of KCs lead to activation of feedback loops that in turn regulate synaptic plasticity. To investigate if the output of the KC is needed for synaptic plasticity the expression of neuronal output blocker in a single KC e.g., Shits (Kitamoto, 2001) or the reduction of neuronal excitability e.g., Kir1.2 (Baines et al., 2001; White et al., 2001) can be used. However, these experiments 
would affect the physiology of the whole cell as current techniques cannot restrict manipulations to single synapses or compartments, yet. Nonetheless, these experiments can indicate the involvement in the mechanism of synaptic de-synchronization.

As a single compartment and synapse manipulation is not yet possible, a bioinformatics approach could investigate the mechanisms behind synaptic de-synchronization. As the connectome studies (Eichler et al., 2017; Takemura et al., 2017) provided anatomical understanding of the circuit, my study can provide and add the physiological basis for such a model. In this model a MB network could be simulated that contains the KCs behaving based on the synaptic physiology measured in this study and the extrinsic circuit anatomy provided by the connectome study. If the network is trained enough to resemble the response profiles of this study, single synaptic contacts can be manipulated and their effect on synchronization investigated. 


\section{Summary}

Learning and memory is a prerequisite for the survival and the appropriate adaptation of behavioral responses in an ever-changing environment. Memories are formed, stored, and retrieved in the brains of animals. The formation of memories leaves traces in the brain, which are formed due to anatomic, physiological, and synaptic alterations in neuronal substrates. Forming and memorizing associations with different stimuli will allow the animal to predict rewarding or punishing conditions in the future. For the detection of environmental stimuli, such as food sources, mates, or harmful substances, olfaction is a commonly used sense. The fruit fly Drosophila melanogaster performs well in olfactory associative learning, assigning valances to former neutral stimuli. This learning performance was attributed to the mushroom bodies, a specialized higher association center in the fly's brain, and also of other arthropods. The mushroom bodies receive heavy olfactory, as well as dopaminergic input, of which the latter conveys mainly aversive and appetitive valence, respectively. The coincidence of odor-induced activation in the mushroom body intrinsic Kenyon cells and the reward- or punishment-induced dopamine release onto these Kenyon cells, is believed to change synaptic plasticity in the mushroom body circuit, leading to the association of both. As higher order brain centers often encode sensory inputs as sparse ensembles of active neurons, which further have a multitude of synapses, it is very challenging to detect memory traces in the brain. Some memory traces were detected in the mushroom body related circuit. However, the synaptic plasticity underlying associative olfactory learning was so far not described for Kenyon cells. Kenyon cells can be subdivided into three main types, which were shown to have certain roles in associative learning and memory. The $y$-type Kenyon cells are mainly involved in short term memory and important for memory acquisition in general.

In the present study, calcium imaging was employed in single $\gamma$-Kenyon cells, to measure odorevoked calcium transients in single synapses, before and shortly after an olfactory aversive associative conditioning. Calcium imaging at the single cell level was accomplished by using mosaic analysis with a repressible cell marker. Single axonal boutons could be identified and monitored in a compartment-specific manner, to analyze synaptic plasticity. The aversive associative conditioning was performed under a 2-photon microscope. After the calcium imaging procedure, flies were subjected to an immunohistochemical protocol to reconstruct single $\mathrm{\gamma}$ Kenyon cells and assign the axonal boutons to their $\mathrm{\gamma}$-lobe compartment. 
In this study it was found, first, that $\psi$-Kenyon cells show compartment-specific odor responses, indicating an expansion of the odor coding space, which is greater than earlier believed. Second, $\gamma$-Kenyon cell synapses de-synchronized in the course of aversive olfactory associative learning, for the odor that was paired with an electric shock. Although the net output of $\psi$-Kenyon cells remained unchanged, synaptic de-synchronization within and across $\gamma$-Kenyon cells tagged stimulus relevant information to those cells. Furthermore, bouton response classes were found across all $y$-lobe compartments, which were rearranged in the course of aversive olfactory associative learning. This rearrangement led to a reduced information output, potentially reducing input to downstream mushroom body output neurons. This form of synaptic desynchronization was now described for the first time in invertebrate mushroom body neurons, showing an essential component of the memory trace left in the brain. Further studies have to show which molecular processes are underlying such a plasticity mechanism and if other Kenyon cell types exhibit similar mechanisms. 


\section{References}

Akalal, D.-B.G., Wilson, C.F., Zong, L., Tanaka, N.K., Ito, K., and Davis, R.L. (2006). Roles for Drosophila mushroom body neurons in olfactory learning and memory. Learning \& Memory 13 , 659-668.

Akalal, D.-B.G., Yu, D., and Davis, R.L. (2010). A Late-Phase, Long-Term M emory Trace Forms in the Neurons of Drosophila Mushroom Bodies after Olfactory Classical Conditioning. Journal of Neuroscience 30, 16699-16708.

Akalal, D.-B.G., Yu, D., and Davis, R.L. (2011). The Long-Term Memory Trace Formed in the Drosophila $\alpha / \beta$ M ushroom Body Neurons Is Abolished in Long-Term M emory M utants. Journal of Neuroscience 31, 5643-5647.

Ashburner, M., and Bonner, J.J. (1979). The induction of gene activity in Drosophila by heat shock. Cell 17, 241-254.

Ashraf, S.I., McLoon, A.L., Sclarsic, S.M., and Kunes, S. (2006). Synaptic Protein Synthesis Associated with Memory Is Regulated by the RISC Pathway in Drosophila. Cell 124, 191-205.

Aso, Y., and Rubin, G.M . (2016). Dopaminergic neurons write and update memories with cell-typespecific rules. ELife 5.

Aso, Y., Grübel, K., Busch, S., Friedrich, A.B., Siwanowicz, I., and Tanimoto, H. (2009). The Mushroom Body of Adult Drosophila Characterized by GAL4 Drivers. Journal of Neurogenetics 23, 156-172.

Aso, Y., Siwanowicz, I., Bräcker, L., Ito, K., Kitamoto, T., and Tanimoto, H. (2010). Specific Dopaminergic Neurons for the Formation of Labile Aversive Memory. Current Biology 20, 14451451.

Aso, Y., Herb, A., Ogueta, M., Siwanowicz, I., Templier, T., Friedrich, A.B., Ito, K., Scholz, H., and Tanimoto, H. (2012). Three Dopamine Pathways Induce Aversive Odor Memories with Different Stability. PLoS Genetics 8, e1002768.

Aso, Y., Hattori, D., Yu, Y., Johnston, R.M., Iyer, N.A., Ngo, T.-T., Dionne, H., Abbott, L.F., Axel, R., Tanimoto, H., et al. (2014a). The neuronal architecture of the mushroom body provides a logic for associative learning. Elife 3, e04577.

Aso, Y., Sitaraman, D., Ichinose, T., Kaun, K.R., Vogt, K., Belliart-Guérin, G., Plaçais, P.-Y., Robie, A.A., Yamagata, N., Schnaitmann, C., et al. (2014b). Mushroom body output neurons encode valence and guide memory-based action selection in Drosophila. ELife 3, e04580.

Baier, A., Wittek, B., and Brembs, B. (2002). Neurobiology of aggression in Drosophila. The Journal of Experimental Biology 205, 1233-1240.

Bailey, C.H., Bartsch, D., and Kandel, E.R. (1996). Toward a molecular definition of long-term memory storage. Proceedings of the National Academy of Sciences 93, 13445-13452. 
Baines, R.A., Uhler, J.P., Thompson, A., Sweeney, S.T., and Bate, M. (2001). Altered Electrical Properties in Drosophila Neurons Developing without Synaptic Transmission. The Journal of Neuroscience 21, 1523-1531.

Barnstedt, O., Owald, D., Felsenberg, J., Brain, R., Moszynski, J.-P., Talbot, C.B., Perrat, P.N., and Waddell, S. (2016). Memory-Relevant Mushroom Body Output Synapses Are Cholinergic. Neuron $89,1237-1247$.

Barth, J., Dipt, S., Pech, U., Hermann, M., Riemensperger, T., and Fiala, A. (2014). Differential Associative Training Enhances Olfactory Acuity in Drosophila melanogaster. Journal of Neuroscience 34, 1819-1837.

Bear, M.F., and Malenka, R.C. (1994). Synaptic plasticity: LTP and LTD. Current Opinion in Neurobiology 4, 389-399.

de Belle, J., and Heisenberg, M. (1994). Associative odor learning in Drosophila abolished by chemical ablation of mushroom bodies. Science 263, 692-695.

Bliss, T.V.P., and Lømo, T. (1973). Long-lasting potentiation of synaptic transmission in the dentate area of the anaesthetized rabbit following stimulation of the perforant path. The Journal of Physiology 232, 331-356.

Blum, A.L., Li, W., Cressy, M., and Dubnau, J. (2009). Short- and Long-Term M emory in Drosophila Require CAM P Signaling in Distinct Neuron Types. Current Biology 19, 1341-1350.

Boquet, I., Hitier, R., Dumas, M., Chaminade, M., and Préat, T. (2000). Central brain postembryonic development in Drosophila: Implication of genes expressed at the interhemispheric junction. Journal of Neurobiology 42, 33-48.

Borst, A., and Theunissen, F.E. (1999). Information theory and neural coding. Nature Neuroscience 2, 947-957.

Boto, T., Louis, T., Jindachomthong, K., Jalink, K., and Tomchik, S.M. (2014). Dopaminergic Modulation of CAMP Drives Nonlinear Plasticity across the Drosophila Mushroom Body Lobes. Current Biology 24, 822-831.

Boutros, M., Kiger, A.A., Armknecht, S., Kerr, K., Hild, M., Koch, B., Hass, S.A., Heidelberg Fly Array Consortium, Paro, R., and Perrimon, N. (2004). Genome-Wide RNAi Analysis of Growth and Viability in Drosophila Cells. Science 303, 832-835.

Bouzaiane, E., Trannoy, S., Scheunemann, L., Plaçais, P.-Y., and Preat, T. (2015). Two Independent Mushroom Body Output Circuits Retrieve the Six Discrete Components of Drosophila Aversive M emory. Cell Reports 11, 1280-1292.

Brand, A.H., and Perrimon, N. (1993). Targeted gene expression as a means of altering cell fates and generating dominant phenotypes. Development 118, 401- 415.

Brown, M.R., Crim, J.W., Arata, R.C., Cai, H.N., Chun, C., and Shen, P. (1999). Identification of a Drosophila brain-gut peptide related to the neuropeptide $Y$ family. Peptides 20, 1035-1042.

de Bruyne, M., Clyne, P.J., and Carlson, J.R. (1999). Odor Coding in a Model Olfactory Organ: The Drosophila M axillary Palp. The Journal of Neuroscience 19, 4520-4532. 
de Bruyne, M., Foster, K., and Carlson, J.R. (2001). Odor Coding in the Drosophila Antenna. Neuron 30, 537-552.

Burke, C.J., and Waddell, S. (2011). Remembering Nutrient Quality of Sugar in Drosophila. Current Biology 21, 746-750.

Burke, C.J., Huetteroth, W., Owald, D., Perisse, E., Krashes, M.J., Das, G., Gohl, D., Silies, M., Certel, S., and Waddell, S. (2012). Layered reward signalling through octopamine and dopamine in Drosophila. Nature 492, 433-437.

Busto, G.U., Cervantes-Sandoval, I., and Davis, R.L. (2010). Olfactory Learning in Drosophila. Physiology 25, 338-346.

Butcher, N.J., Friedrich, A.B., Lu, Z., Tanimoto, H., and M einertzhagen, I.A. (2012). Different classes of input and output neurons reveal new features in microglomeruli of the adult Drosophila mushroom body calyx. The Journal of Comparative Neurology 520, 2185-2201.

Byers, D., Davis, R.L., and Kiger, J.A. (1981). Defect in cyclic AMP phosphodiesterase due to the dunce mutation of learning in Drosophila melanogaster. Nature 289, 79-81.

Byrne, J.H., and Kandel, E.R. (1996). Presynaptic Facilitation Revisited: State and Time Dependence. The Journal of Neuroscience 16, 425-435.

Cajal, S.R.Y. (1894). The Croonian Lecture: La Fine Structure des Centres Nerveux. Proceedings of the Royal Society of London 55, 444-468.

Caporale, N., and Dan, Y. (2008). Spike Timing-Dependent Plasticity: A Hebbian Learning Rule. Annual Review of Neuroscience 31, 25-46.

Carew, T., Hawkins, R., and Kandel, E. (1983). Differential classical conditioning of a defensive withdrawal reflex in Aplysia californica. Science 219, 397-400.

Carew, T.J., Walters, E.T., and Kandel, E.R. (1981). Classical conditioning in a Simple Withdrawal Reflex in Aplysia californica. The Journal of Neuroscience 1, 1426-1437.

Caron, S.J.C., Ruta, V., Abbott, L.F., and Axel, R. (2013). Random convergence of olfactory inputs in the Drosophila mushroom body. Nature 497, 113-117.

Cassenaer, S., and Laurent, G. (2007). Hebbian STDP in mushroom bodies facilitates the synchronous flow of olfactory information in locusts. Nature 448, 709-713.

Castellucci, V.F., Nairn, A., Greengard, P., Schwartz, J.H., and Kandel, E.R. (1982). Inhibitor of Adenosine 3':5'-Monophosphate-Dependent Protein Kinase Blocks Presynaptic Facilitation in Aplysia. The Journal of Neuroscience 2, 1673-1681.

Castellucci, V.F., Blumenfeld, H., Goelet, P., and Kandel, E.R. (1989). Inhibitor of protein synthesis blocks longterm behavioral sensitization in the isolated gill-withdrawal reflex of Aplysia. Journal of Neurobiology 20, 1-9.

Cedar, H., Kandel, E.R., and Schwartz, J.H. (1972). Cyclic Adenosine Monophosphate in the Nervous System of Aplysia californica: I. Increased synthesis in response to synaptic stimulation. The Journal of General Physiology 60, 558-569. 
Cervantes-Sandoval, I., and Davis, R.L. (2012). Distinct Traces for Appetitive versus Aversive Olfactory Memories in DPM Neurons of Drosophila. Current Biology 22, 1247-1252.

Cervantes-Sandoval, I., Martin-Pena, A., Berry, J.A., and Davis, R.L. (2013). System-Like Consolidation of Olfactory Memories in Drosophila. Journal of Neuroscience 33, 9846-9854.

Chabaud, M.-A., Devaud, J.-M., Pham-Delègue, M.-H., Preat, T., and Kaiser, L. (2006). Olfactory conditioning of proboscis activity in Drosophila melanogaster. Journal of Comparative Physiology A 192, 1335-1348.

Chalfie, M., Tu, Y., Euskirschen, G., Ward, W.W., and Prasher, D.C. (1994). Green Fluorescent Protein as a M arker for Gene Expression. Science 263, 802-805.

Chen, C.N., Denome, S., and Davis, R.L. (1986). Molecular analysis of cDNA clones and the corresponding genomic coding sequences of the Drosophila dunce+gene, the structural gene for CAM P phosphodiesterase. Proceedings of the National Academy of Sciences 83, 9313-9317.

Chen, S., Lee, A.Y., Bowens, N.M., Huber, R., and Kravitz, E.A. (2002). Fighting fruit flies: A model system for the study of aggression. Proceedings of the National Academy of Sciences 99, 56645668.

Choi, G.B., Stettler, D.D., Kallman, B.R., Bhaskar, S.T., Fleischmann, A., and Axel, R. (2011). Driving Opposing Behaviors with Ensembles of Piriform Neurons. Cell 146, 1004-1015.

Claridge-Chang, A., Roorda, R.D., Vrontou, E., Sjulson, L., Li, H., Hirsh, J., and Miesenböck, G. (2009). Writing Memories with Light-Addressable Reinforcement Circuitry. Cell 139, 405-415.

Cognigni, P., Felsenberg, J., and Waddell, S. (2018). Do the right thing: neural network mechanisms of memory formation, expression and update in Drosophila. Current Opinion in Neurobiology 49, 51-58.

Cohn, R., Morantte, I., and Ruta, V. (2015). Coordinated and Compartmentalized Neuromodulation Shapes Sensory Processing in Drosophila. Cell 163, 1742-1755.

Connolly, J.B., Roberts, I.J.H., Armstrong, J.D., Kaiser, K., Forte, M., Tully, T., and O'Kane, C.J. (1996). Associative Learning Disrupted by Impaired Gs Signaling in Drosophila Mushroom Bodies. Science 274, 2104-2107.

Couto, A., Alenius, M., and Dickson, B.J. (2005). Molecular, Anatomical, and Functional Organization of the Drosophila Olfactory System. Current Biology 15, 1535-1547.

Crittenden, J.R., Skoulakis, E.M.C., Han, K.-A., Kalderon, D., and Davis, R.L. (1998). Tripartite M ushroom Body Architecture Revealed by Antigenic Markers. Learning \& Memory 5, 38-51.

Das, G., Klappenbach, M., Vrontou, E., Perisse, E., Clark, C.M., Burke, C.J., and Waddell, S. (2014). Drosophila Learn Opposing Components of a Compound Food Stimulus. Current Biology 24, 17231730.

Davis, R.L. (1993). Mushroom bodies and Drosophila learning. Neuron 11, 1-14.

Davis, R.L. (2005). Olfactory Memory Formation in Drosophila: From Molecular to Systems Neuroscience. Annual Review of Neuroscience 28, 275-302.

Davis, R.L. (2011). Traces of Drosophila Memory. Neuron 70, 8-19. 
Davis, R.L., and Kiger, J.A. (1981). Dunce mutants of Drosophila melanogaster: mutants defective in the cyclic AM P phosphodiesterase enzyme system. The Journal of Cell Biology 90, 101-107.

Dejianne, D., McGuire, T.R., and Pruzan-Hotchkiss, A. (1985). Conditioned Suppression of Proboscis Extension in Drosophila melanogaster. Journal of Comparative Psychology 99, 74-80.

Denk, W., Sugimori, M., and Llinas, R. (1995). Two types of calcium response limited to single spines in cerebellar Purkinje cells. Proceedings of the National Academy of Sciences 92, 82798282.

Dill, M., Wolf, R., and Heisenberg, M. (1993). Visual pattern recognition in Drosophila involves retinotopic matching. Nature $365,751-753$.

Dubnau, J., Grady, L., Kitamoto, T., and Tully, T. (2001). Disruption of neurotransmission in Drosophila mushroom body blocks retrieval but not acquisition of memory. 411, 5 .

Dudai, Y. (2004). The Neurobiology of Consolidations, Or, How Stable is the Engram? Annual Review of Psychology 55, 51-86.

Dudai, Y., Jan, Y.N., Byers, D., Quinn, W.G., and Benzer, S. (1976). Dunce, a mutant of Drosophila deficient in learning. Proceedings of the National Academy of Sciences 73, 1684- 1688.

Dujardin, F. (1850). Mémoire sur le système nerveux des insectes. Annales Des Sciences Naturelles, Zoologie 14, 195-206.

Edwards, A.C., Zwarts, L., Yamamoto, A., Callaerts, P., and Mackay, T.F. (2009). M utations in many genes affect aggressive behavior in Drosophila melanogaster. BM C Biology 7, 29.

Eichler, K., Li, F., Litwin-Kumar, A., Park, Y., Andrade, I., Schneider-Mizell, C.M., Saumweber, T., Huser, A., Eschbach, C., Gerber, B., et al. (2017). The complete connectome of a learning and memory centre in an insect brain. Nature 548, 175-182.

Feinberg, E.H., VanHoven, M.K., Bendesky, A., Wang, G., Fetter, R.D., Shen, K., and Bargmann, C.I. (2008). GFP Reconstitution Across Synaptic Partners (GRASP) Defines Cell Contacts and Synapses in Living Nervous Systems. Neuron 57, 353-363.

Ferris, J., Ge, H., Liu, L., and Roman, G. (2006). G(0) signaling is required for Drosophila associative learning. Nature Neuroscience 9, 1036-1040.

Fiala, A., Spall, T., Diegelmann, S., Eisermann, B., Sachse, S., Devaud, J.-M., Buchner, E., and Galizia, C.G. (2002). Genetically Expressed Cameleon in Drosophila melanogaster Is Used to Visualize Olfactory Information in Projection Neurons. Current Biology 12, 1877-1884.

Fischer, J.A., Giniger, E., Maniatis, T., and Ptashne, M. (1988). GAL4 activates transcription in Drosophila. Nature 332, 853-856.

Fişek, M., and Wilson, R.I. (2014). Stereotyped connectivity and computations in higher-order olfactory neurons. Nature Neuroscience 17, 280-288.

Foster, J.L., Higgins, G.C., and Jackson, F.R. (1988). Cloning, Sequence, and Expression of the Drosophila CAMP-dependent Protein Kinase Catalytic Subunit Gene. The Journal of Biological Chemistry 263, 1676-1681. 
Fouquet, W., Owald, D., Wichmann, C., M ertel, S., Depner, H., Dyba, M., Hallermann, S., Kittel, R.J., Eimer, S., and Sigrist, S.J. (2009). Maturation of active zone assembly by Drosophila Bruchpilot. The Journal of Cell Biology 186, 129-145.

Fresquet, N., Fournier, D., and Gauthier, M. (1998). A New Attempt to Assess the Effect of Learning Processes on the Cholinergic System: Studies on Fruitflies and Honeybees. Comparative Biochemistry and Physiology Part B: Biochemistry and M olecular Biology 119, 349-353.

Galili, D.S., Dylla, K.V., Lüdke, A., Friedrich, A.B., Yamagata, N., Wong, J.Y.H., Ho, C.H., Szyszka, P., and Tanimoto, H. (2014). Converging Circuits Mediate Temperature and Shock Aversive Olfactory Conditioning in Drosophila. Current Biology 24, 1712-1722.

Gao, Q., Yuan, B., and Chess, A. (2000). Convergent projections of Drosophila olfactory neurons to specific glomeruli in the antennal lobe. Nature Neuroscience 3, 780-785.

Gerber, B., Tanimoto, H., and Heisenberg, M. (2004). An engram found? Evaluating the evidence from fruit flies. Current Opinion in Neurobiology 14, 737-744.

Gervasi, N., Tchénio, P., and Preat, T. (2010). PKA Dynamics in a Drosophila Learning Center: Coincidence Detection by Rutabaga Adenylyl Cyclase and Spatial Regulation by Dunce Phosphodiesterase. Neuron 65, 516-529.

Golic, K. (1991). Site-specific recombination between homologous chromosomes in Drosophila. Science 252, 958-961.

Golic, K.G., and Lindquist, S. (1989). The FLP recombinase of yeast catalyzes site-specific recombination in the Drosophila genome. Cell 59, 499-509.

Goodwin, S.F., Vecchio, M.D., Velinzon, K., Hogel, C., Russell, S.R.H., Tully, T., and Kaiser, K. (1997). Defective Learning in Mutants of the Drosophila Gene for a Regulatory Subunit of CAMPDependent Protein Kinase. The Journal of Neuroscience 17, 8817-8827.

Gordon, M.D., and Scott, K. (2009). Motor Control in a Drosophila Taste Circuit. Neuron 61, 373384.

Gormenzano, I., Schneiderman, N., Deaux, E., and Fuentes, I. (1962). Nictitating Membrane: Classical Conditioning and Extinction in the Albino Rabbit. Science 138, 33-34.

Goyal, R.K., and Chaudhury, A. (2013). Structure activity relationship of synaptic and junctional neurotransmission. Autonomic Neuroscience 176, 11-31.

Grabe, V., and Sachse, S. (2018). Fundamental principles of the olfactory code. Biosystems 164, 94-101.

Grabe, V., Strutz, A., Baschwitz, A., Hansson, B.S., and Sachse, S. (2015). Digital in vivo 3D atlas of the antennal lobe of Drosophila melanogaster: In Vivo Atlas of Drosophila Antennal Lobe. Journal of Comparative Neurology 523, 530-544.

Grant, D.A., and Adams, J.K. (1944). "Alpha" conditioning in the eyelid. Journal of Experimental Psychology 34, 136-142.

Gruntman, E., and Turner, G.C. (2013). Integration of the olfactory code across dendritic claws of single mushroom body neurons. Nature Neuroscience 16, 1821-1829. 
Guo, A., and Götz, K.G. (1997). Association of visual objects and olfactory cues in Drosophila. Learning \& Memory 4, 192-204.

Gupta, N., and Stopfer, M. (2012). Functional Analysis of a Higher Olfactory Center, the Lateral Horn. Journal of Neuroscience 32, 8138-8148.

Hall, J. (1994). The mating of a fly. Science 264, 1702-1714.

Hall, A., and Lalli, G. (2010). Rho and Ras GTPases in Axon Growth, Guidance, and Branching. Cold Spring Harbor Perspectives in Biology 2, a001818.

Hallem, E.A., and Carlson, J.R. (2004). The odor coding system of Drosophila. Trends in Genetics 20, 453-459.

Hammer, M. (1993). An identified neuron mediates the unconditioned stimulus in associative olfactory learning in honeybees. Nature 336, 59-63.

Han, K.-A., Millar, N.S., Grotewiel, M.S., and Davis, R.L. (1996). DAMB, a Novel Dopamine Receptor Expressed Specifically in Drosophila Mushroom Bodies. Neuron 16, 1127-1135.

Han, P.-L., Levin, L.R., Reed, R.R., and Davis, R.L. (1992). Preferential Expression of the Drosophila rutaga Gene in M ushroom Bodies, Neural Centers for Learning in Insects. Neuron 9, 619-627.

Harvey, C.D., and Svoboda, K. (2007). Locally dynamic synaptic learning rules in pyramidal neuron dendrites. Nature 450, 1195-1200.

Harvey, C.D., Yasuda, R., Zhong, H., and Svoboda, K. (2008). The Spread of Ras Activity Triggered by Activation of a Single Dendritic Spine. Science 321, 136-140.

Hawkins, R.D. (1984). A Cellular Mechanism of Classical Conditioning in Aplysia. Journal of Experimental Biology 112, 113-128.

Haynes, P.R., Christmann, B.L., and Griffith, L.C. (2015). A single pair of neurons links sleep to memory consolidation in Drosophila melanogaster. ELife 4, e03868.

Hebb, D.O. (1949). The Organization of Behavior: A Neurophysiological Theory (New York: JOHN WILEY \& Sons, Inc.).

Heim, R., Cubitt, A.B., and Tsien, R.Y. (1995). Improved green fluorescence. Nature 373, 663-664.

Heimbeck, G., Bugnon, V., Gendre, N., Keller, A., and Stocker, R.F. (2001). A central neural circuit for experience-independent olfactory and courtship behavior in Drosophila melanogaster. Proceedings of the National Academy of Sciences 98, 15336-15341.

Heisenberg, M. (2003). Mushroom body memoir: from maps to models. Nature Reviews Neuroscience 4, 266-275.

Heisenberg, M., Borst, A., Wagner, S., and Byers, D. (1985). Drosophila Mushroom Body M utants are Deficient in Olfactory Learning: Research Papers. Journal of Neurogenetics 2, 1-30.

Hendricks, J.C., Finn, S.M., Panckeri, K.A., Chavkin, J., Williams, J.A., Sehgal, A., and Pack, A.I. (2000). Rest in Drosophila Is a Sleep-like State. Neuron 25, 129-138. 
Hescheler, J., Rosenthal, W., Trautwein, W., and Schultz, G. (1987). The GTP-binding protein, Go, regulates neuronal calcium channels. Nature 325, 445-447.

Hige, T. (2018). What can tiny mushrooms in fruit flies tell us about learning and memory? Neuroscience Research 129, 8-16.

Hige, T., Aso, Y., Modi, M.N., Rubin, G.M., and Turner, G.C. (2015a). Heterosynaptic Plasticity Underlies Aversive Olfactory Learning in Drosophila. Neuron 88, 985-998.

Hige, T., Aso, Y., Rubin, G.M., and Turner, G.C. (2015b). Plasticity-driven individualization of olfactory coding in mushroom body output neurons. Nature 526, 258-262.

Holland, P.C., and Bouton, M.E. (1999). Hippocampus and context in classical conditioning. Current Opinion in Neurobiology 9, 195-202.

Holliday, M., and Hirsch, J. (1986). Excitatory Conditioning of Individual Drosophila melanogaster. Journal of Experimental Psychology 12, 131-142.

Honegger, K.S., Campbell, R.A.A., and Turner, G.C. (2011). Cellular-Resolution Population Imaging Reveals Robust Sparse Coding in the Drosophila Mushroom Body. Journal of Neuroscience 31, 11772-11785.

Huetteroth, W., Perisse, E., Lin, S., Klappenbach, M., Burke, C., and Waddell, S. (2015). Sweet Taste and Nutrient Value Subdivide Rewarding Dopaminergic Neurons in Drosophila. Current Biology 25, 751-758.

Ikeda, S.R. (1996). Voltage-dependent modulation of N-type calcium channels by G-protein $\beta \gamma$ subunits. Nature 380, 255-258.

Inada, K., Tsuchimoto, Y., and Kazama, H. (2017). Origins of Cell-Type-Specific Olfactory Processing in the Drosophila Mushroom Body Circuit. Neuron 95, 357-367.e4.

Ito, K., and Hotta, Y. (1992). Proliferation Pattern of Postembryonic Neuroblasts in the Brain of Drosophila melanogaster. Developmental Biology 149, 134-148.

Ito, K., Suzuki, K., Estes, P., Ramaswami, M., Yamamoto, D., and Strausfeld, N.J. (1998). The Organization of Extrinsic Neurons and Their Implications in the Functional Roles of the Mushroom Bodies in Drosophila melanogaster M eigen. Learning \& M emory 5, 52- 77.

Janak, P.H., and Tye, K.M. (2015). From circuits to behaviour in the amygdala. Nature 517, 284292.

Jefferis, G.S.X.E., Marin, E.C., Stocker, R.F., and Luo, L. (2001). Target neuron prespecification in the olfactory map of Drosophila. Nature 414, 204-208.

Jefferis, G.S.X.E., Potter, C.J., Chan, A.M ., Marin, E.C., Rohlfing, T., M aurer, C.R., and Luo, L. (2007). Comprehensive Maps of Drosophila Higher Olfactory Centers: Spatially Segregated Fruit and Pheromone Representation. Cell 128, 1187-1203.

Johard, H.A.D., Enell, L.E., Gustafsson, E., Trifilieff, P., Veenstra, J.A., and Nässel, D.R. (2008). Intrinsic neurons of Drosophila mushroom bodies express short neuropeptide F: Relations to extrinsic neurons expressing different neurotransmitters. The Journal of Comparative Neurology $507,1479-1496$. 
Joiner, W.J., Crocker, A., White, B.H., and Sehgal, A. (2006). Sleep in Drosophila is regulated by adult mushroom bodies. Nature 441, 757-760.

Josselyn, S.A., Köhler, S., and Frankland, P.W. (2015). Finding the engram. Nature Reviews Neuroscience 16, 521-534.

Josselyn, S.A., Köhler, S., and Frankland, P.W. (2017). Heroes of the Engram. The Journal of Neuroscience 37, 4647-4657.

Kahsai, L., and Zars, T. (2011). Learning and Memory in Drosophila: Behavior, Genetics, and Neural Systems. International Review of Neurobiology 99, 139-167.

Kandel, E.R. (2001). The Molecular Biology of Memory Storage: A Dialogue Between Genes and Synapses. Science 294, 1030-1038.

Kandel, E.R. (2012). The molecular biology of memory: CAMP, PKA, CRE, CREB-1, CREB-2, and CPEB. Molecular Brain 5, 14.

Kandel, E.R., Dudai, Y., and M ayford, M.R. (2014). The Molecular and Systems Biology of Memory. Cell 157, 163-186.

Katz, B., and Miledi, R. (1965). The Effect of Calcium on Acetylcholine Release from M otor Nerve Terminals. Proceedings of the Royal Society B: Biological Sciences 161, 496-503.

Kaun, K.R., and Rothenfluh, A. (2017). Dopaminergic rules of engagement for memory in Drosophila. Current Opinion in Neurobiology 43, 56-62.

Kaun, K.R., Azanchi, R., Maung, Z., Hirsh, J., and Heberlein, U. (2011). A Drosophila model for alcohol reward. Nature Neuroscience 14, 612-619.

Keene, A.C., and Waddell, S. (2007). Drosophila olfactory memory: single genes to complex neural circuits. Nature Reviews Neuroscience 8, 341-354.

Keene, A.C., Stratmann, M., Keller, A., Perrat, P.N., Vosshall, L.B., and Waddell, S. (2004). Diverse Odor-Conditioned Memories Require Uniquely Timed Dorsal Paired Medial Neuron Output. Neuron 44, 521-533.

Keene, A.C., Krashes, M.J., Leung, B., Bernard, J.A., and Waddell, S. (2006). Drosophila Dorsal Paired Medial Neurons Provide a General M echanism for Memory Consolidation. Current Biology 16, 1524-1530.

Kenyon, F.C. (1896a). The brain of the bee. A preliminary contribution to the morphology of the nervous system of the arthropoda. Journal of Comparative Neurology 6, 133-210.

Kenyon, F.C. (1896b). The Meaning and Structure of the So-Called "Mushroom Bodies" of the Hexapod Brain. The American Naturalist 30, 643-650.

Kim, Y.-C., Lee, H.-G., Seong, C.-S., and Han, K.-A. (2003). Expression of a D1 dopamine receptor dDA1/DmDOP1 in the central nervous system of Drosophila melanogaster. Gene Expression Patterns 3, 237-245.

Kim, Y.-C., Lee, H.-G., and Han, K.-A. (2007). D1 Dopamine Receptor dDA1 Is Required in the Mushroom Body Neurons for Aversive and Appetitive Learning in Drosophila. Journal of Neuroscience 27, 7640-7647. 
Kirkhart, C., and Scott, K. (2015). Gustatory Learning and Processing in the Drosophila Mushroom Bodies. Journal of Neuroscience 35, 5950-5958.

Kitamoto, T. (2001). Conditional Modification of Behavior in Drosophila by Targeted Expression of a Temperature-sensitive shibire Allele in Defined Neurons. Journal of Neurobiology 47, 81-92.

Klapoetke, N.C., Murata, Y., Kim, S.S., Pulver, S.R., Birdsey-Benson, A., Cho, Y.K., Morimoto, T.K., Chuong, A.S., Carpenter, E.J., Tian, Z., et al. (2014). Independent optical excitation of distinct neural populations. Nature M ethods 11, 338-346.

Knapek, S., Kahsai, L., Winther, A.M.E., Tanimoto, H., and Nassel, D.R. (2013). Short Neuropeptide F Acts as a Functional Neuromodulator for Olfactory Memory in Kenyon Cells of Drosophila Mushroom Bodies. Journal of Neuroscience 33, 5340-5345.

Konorski, J. (1948). Conditioned Reflexes and Neuron Organisation (Cambridge: Cambridge University Press).

Krashes, M.J., and Waddell, S. (2008). Rapid Consolidation to a radish and Protein SynthesisDependent Long-Term Memory after Single-Session Appetitive Olfactory Conditioning in Drosophila. J ournal of Neuroscience 28, 3103-3113.

Krashes, M.J., Keene, A.C., Leung, B., Armstrong, J.D., and Waddell, S. (2007). Sequential Use of Mushroom Body Neuron Subsets during Drosophila Odor Memory Processing. Neuron 53, 103115.

Kurusu, M., Awasaki, T., M asuda-Nakagawa, L.M., Kawauchi, H., Ito, K., and Furukubo-Tokunaga, K. (2002). Early development of mushroom bodies. Development 129, 409-419.

Kuwabara, M. (1957). Bildung des bedingten Reflexes von Pavlovs Typus bei der Honigbiene, Apis mellifica. Journal of the Faculty of Science Hokkaido UniversitySeries VI. Zoology 13, 458-464.

Lai, S.-L., and Lee, T. (2006). Genetic mosaic with dual binary transcriptional systems in Drosophila. Nature Neuroscience 9, 703-709.

Larsson, M.C., Domingos, A.I., Jones, W.D., Chiappe, M.E., Amrein, H., and Vosshall, L.B. (2004). Or83b Encodes a Broadly Expressed Odorant Receptor Essential for Drosophila Olfaction. Neuron 43, 703-714.

Lee, T., and Luo, L. (1999). Mosaic analysis with a repressible cell marker for studies of gene function in neuronal morphogenesis. Neuron 22, 451-461.

Lee, P.-T., Lin, H.-W., Chang, Y.-H., Fu, T.-F., Dubnau, J., Hirsh, J., Lee, T., and Chiang, A.-S. (2011). Serotonin-mushroom body circuit modulating the formation of anesthesia-resistant memory in Drosophila. Proceedings of the National Academy of Sciences 108, 13794-13799.

Lee, T., Lee, A., and Luo, L. (1999). Development of the Drosophila mushroom bodies: sequential generation of three distinct types of neurons from a neuroblast. Development 126, 4065-4076.

Leiss, F., Groh, C., Butcher, N.J., Meinertzhagen, I.A., and Tavosanis, G. (2009). Synaptic organization in the adult Drosophila mushroom body calyx. The Journal of Comparative Neurology $517,808-824$.

Leung, H.-T., Dale Branton, W., Phillips, H.S., Jan, L., and Byerly, L. (1989). Spider Toxins Selectively Block Calcium Currents in Drosophila. Neuron 3, 767-772. 
Levin, L.R., Han, P.-L., Feinstein, P.G., Davis, R.L., and Reed, R.R. (1992). The Drosophila Learning and Memory Gene rutabaga Encodes a Ca2+/Calmodulin-Responsive Adenylyl Cyclase. Cell 68, 479-489.

Lin, A.C., Bygrave, A.M., de Calignon, A., Lee, T., and M iesenböck, G. (2014a). Sparse, decorrelated odor coding in the mushroom body enhances learned odor discrimination. Nature Neuroscience $17,559-568$.

Lin, H.-H., Lai, J.S.-Y., Chin, A.-L., Chen, Y.-C., and Chiang, A.-S. (2007). A Map of Olfactory Representation in the Drosophila Mushroom Body. Cell 128, 1205-1217.

Lin, S., Owald, D., Chandra, V., Talbot, C., Huetteroth, W., and Waddell, S. (2014b). Neural correlates of water reward in thirsty Drosophila. Nature Neuroscience 17, 1536-1542.

Littleton, J.T., Stern, M., Perin, M., and Bellen, H.J. (1994). Calcium dependence of neurotransmitter release and rate of spontaneous vesicle fusions are altered in Drosophila synaptotagmin mutants. Proceedings of the National Academy of Sciences 91, 10888-10892.

Liu, X., and Davis, R.L. (2009). The GABAergic anterior paired lateral neuron suppresses and is suppressed by olfactory learning. Nature Neuroscience 12, 53-59.

Liu, C., Plaçais, P.-Y., Yamagata, N., Pfeiffer, B.D., Aso, Y., Friedrich, A.B., Siwanowicz, I., Rubin, G.M., Preat, T., and Tanimoto, H. (2012). A subset of dopamine neurons signals reward for odour memory in Drosophila. Nature 488, 512-516.

Liu, G., Seiler, H., Wen, A., Zars, T., Ito, K., Wolf, R., Heisenberg, M., and Liu, L. (2006). Distinct memory traces for two visual features in the Drosophila brain. Nature 439, 551-556.

Liu, L., Wolf, R., Ernst, R., and Heisenberg, M. (1999). Context generalization in Drosophila visual learning requires the mushroom bodies. Nature 400, 753-756.

Liu, Q., Yang, X., Tian, J., Gao, Z., Wang, M., Li, Y., and Guo, A. (2016). Gap junction networks in mushroom bodies participate in visual learning and memory in Drosophila. ELife 5.

Livingstone, M.S., and Tempel, B.L. (1983). Genetic dissection of monoamine neurotransmitter synthesis in Drosophila. Nature 305, 67-70.

Livingstone, M.S., Sziber, P.P., and Quinn, W.G. (1984). Loss of calcium/calmodulin responsiveness in adenylate cyclase of rutabaga, a Drosophila learning mutant. Cell 37, 205-215.

Luan, H., Peabody, N.C., Vinson, C.R., and White, B.H. (2006). Refined Spatial Manipulation of Neuronal Function by Combinatorial Restriction of Transgene Expression. Neuron 52, 425-436.

Lüdke, A., Raiser, G., Nehrkorn, J., Herz, A.V.M., Galizia, C.G., and Szyszka, P. (2018). Calcium in Kenyon Cell Somata as a Substrate for an Olfactory Sensory Memory in Drosophila. Frontiers in Cellular Neuroscience 12, 128.

Luo, S.X., Axel, R., and Abbott, L.F. (2010). Generating sparse and selective third-order responses in the olfactory system of the fly. Proceedings of the National Academy of Sciences 107, 1071310718.

Ma, J., and Ptashne, M. (1987). The carboxy-terminal 30 amino acids of GAL4 are recognized by GAL80. Cell 50, 137-142. 
Macpherson, L.J., Zaharieva, E.E., Kearney, P.J., Alpert, M.H., Lin, T.-Y., Turan, Z., Lee, C.-H., and Gallio, M. (2015). Dynamic labelling of neural connections in multiple colours by trans-synaptic fluorescence complementation. Nature Communications 6, 10024.

Madalan, A., Yang, X., Ferris, J., Zhang, S., and Roman, G. (2012). G(0) activation is required for both appetitive and aversive memory acquisition in Drosophila. Learning \& M emory 19, 26-34.

Manoli, D.S., Foss, M., Villella, A., Taylor, B.J., Hall, J.C., and Baker, B.S. (2005). Male-specific fruitless specifies the neural substrates of Drosophila courtship behaviour. Nature 436, 395-400.

Mao, Z., and Davis, R.L. (2009). Eight different types of dopaminergic neurons innervate the Drosophila mushroom body neuropil: anatomical and physiological heterogeneity. Frontiers in Neural Circuits 3, 5.

Marder, E., Gutierrez, G.J., and Nusbaum, M.P. (2017). Complicating Connectomes: Electrical Coupling Creates Parallel Pathways and Degenerate Circuit Mechanisms. Developmental Neurobiology 77, 597-609.

Maren, S. (2001). Neurobiology of Pavlovian Fear Conditioning. Annual Review of Neuroscience 24, 897-931.

Marin, E.C., Jefferis, G.S.X.., Komiyama, T., Zhu, H., and Luo, L. (2002). Representation of the Glomerular Olfactory Map in the Drosophila Brain. Cell 109, 243-255.

Martin, J.-R., Ernst, R., and Heisenberg, M . (1998). Mushroom Bodies Suppress Locomotor Activity in Drosophila melanogaster. Learning \& M emory 5, 179-191.

Martin, S.J., Grimwood, P.D., and Morris, R.G.M. (2000). Synaptic Plasticity and Memory: An Evaluation of the Hypothesis. Annual Review of Neuroscience 23, 649-711.

Masek, P., and Scott, K. (2010). Limited taste discrimination in Drosophila. Proceedings of the National Academy of Sciences 107, 14833-14838.

Masse, N.Y., Turner, G.C., and Jefferis, G.S.X.E. (2009). Olfactory Information Processing in Drosophila. Current Biology 19, R700-R713.

M atz, M.V., Fradkov, A.F., Labas, Y.A., Savitsky, A.P., Zaraisky, A.G., Markelov, M .L., and Lukyanov, S.A. (1999). Fluorescent proteins from nonbioluminescent Anthozoa species. Nature Biotechnology 17, 969-973.

M cBride, S.M.., Giuliani, G., Choi, C., Krause, P., Correale, D., Watson, K., Baker, G., and Siwicki, K.K. (1999). Mushroom Body Ablation Impairs Short-Term Memory and Long-Term Memory of Courtship Conditioning in Drosophila melanogaster. Neuron 24, 967-977.

McGuire, S.E. (2001). The Role of Drosophila Mushroom Body Signaling in Olfactory Memory. Science 293, 1330-1333.

M cGuire, S.E. (2003). Spatiotemporal Rescue of Memory Dysfunction in Drosophila. Science 302, 1765-1768.

M cGuire, S.E., Deshazer, M., and Davis, R.L. (2005). Thirty years of olfactory learning and memory research in Drosophila melanogaster. Progress in Neurobiology 76, 328- 347. 
Médioni, J., Cadieu, N., and Vaysse, G. (1978). Divergent selection for the rapid acquisition of conditioning in Drosophila (Drosophila melanogaster). Comptes Rendus Des Seances de La Societe de Biologie et de Ses Filiales 172, 961-967.

Menzel, R. (2012). The honeybee as a model for understanding the basis of cognition. Nature Reviews Neuroscience 13, 758-768.

Menzel, R., and Müller, U. (1996). Learning and Memory in Honeybees: From Behavior to Neural Substrates. Annual Review of Neuroscience 19, 379-404.

M iesenböck, G., De Angelis, D.A., and Rothman, J.E. (1998). Visualizing secretion and synaptic transmission with pH-sensitive green fluorescent proteins. Nature 394, 192-195.

Miyawaki, A., Griesbeck, O., Heim, R., and Tsien, R.Y. (1999). Dynamic and quantitative Ca2+ measurements using improved cameleons. Proceedings of the National Academy of Sciences 96, 2135-2140.

Moser, M.-B., Rowland, D.C., and Moser, E.I. (2015). Place Cells, Grid Cells, and Memory. Cold Spring Harbor Perspectives in Biology 7, a021808.

Murtagh, F., and Contreras, P. (2012). Algorithms for hierarchical clustering: an overview. Wiley Interdisciplinary Reviews: Data M ining and Knowledge Discovery 2, 86-97.

M urtagh, F., and Contreras, P. (2017). Algorithms for hierarchical clustering: an overview, II. Wiley Interdisciplinary Reviews: Data M ining and Knowledge Discovery 7, e1219.

Murthy, M., Fiete, I., and Laurent, G. (2008). Testing Odor Response Stereotypy in the Drosophila Mushroom Body. Neuron 59, 1009-1023.

Nakagawa, T., and Vosshall, L.B. (2009). Controversy and consensus: noncanonical signaling mechanisms in the insect olfactory system. Current Opinion in Neurobiology 19, 284-292.

Nakai, J., Ohkura, M., and Imoto, K. (2001). A high signal-to-noise Ca2+ probe composed of a single green fluorescent protein. Nature Biotechnology 19, 137-141.

Nayak, S.V., and Singh, R.N. (1985). Primary sensory projections from the labella to the brain of Drosophila melanogaster Meigen (Diptera: Drosophilidae). International Journal of Insect M orphology and Embryology 14, 115-129.

Neve, K.A., Seamans, J.K., and Trantham-Davidson, H. (2004). Dopamine Receptor Signaling. Journal of Receptors and Signal Transduction 24, 165-205.

Ng, M., Roorda, R.D., Lima, S.Q., Zemelman, B.V., Morcillo, P., and Miesenböck, G. (2002). Transmission of Olfactory Information between Three Populations of Neurons in the Antennal Lobe of the Fly. Neuron 36, 463-474.

Nighorn, A., Healy, M.J., and Davis, R.L. (1991). The cyclic AM P phosphodiesterase encoded by the Drosophila dunce gene is concentrated in the mushroom body neuropil. Neuron 6, 455-467.

Ofstad, T.A., Zuker, C.S., and Reiser, M.B. (2011). Visual place learning in Drosophila melanogaster. Nature 474, 204-207.

Olsen, S.R., and Wilson, R.I. (2008). Lateral presynaptic inhibition mediates gain control in an olfactory circuit. Nature 452, 956-960. 
Olsen, S.R., Bhandawat, V., and Wilson, R.I. (2010). Divisive Normalization in Olfactory Population Codes. Neuron 66, 287-299.

Olshausen, B., and Field, D. (2004). Sparse coding of sensory inputs. Current Opinion in Neurobiology 14, 481-487.

Owald, D., and Waddell, S. (2015). Olfactory learning skews mushroom body output pathways to steer behavioral choice in Drosophila. Current Opinion in Neurobiology 35, 178-184.

Owald, D., Felsenberg, J., Talbot, C.B., Das, G., Perisse, E., Huetteroth, W., and Waddell, S. (2015). Activity of Defined Mushroom Body Output Neurons Underlies Learned Olfactory Behavior in Drosophila. Neuron 86, 417-427.

Pai, T.-P., Chen, C.-C., Lin, H.-H., Chin, A.-L., Lai, J.S.-Y., Lee, P.-T., Tully, T., and Chiang, A.-S. (2013). Drosophila ORB protein in two mushroom body output neurons is necessary for long-term memory formation. Proceedings of the National Academy of Sciences 110, 7898-7903.

Parnas, M., Lin, A.C., Huetteroth, W., and Miesenböck, G. (2013). Odor Discrimination in Drosophila: From Neural Population Codes to Behavior. Neuron 79, 932-944.

Pascual, A., and Preat, T. (2001). Localization of Long-Term Memory Within the Drosophila Mushroom Body. Science 294, 1115-1117.

Patterson, M.M., Cegavske, C.F., and Thompson, R.F. (1973). Spinal conditioning. Bulletin of the Psychonomic Society 1, 139-140.

Pavlov, I.P. (1906). The Scientific Investigation of the Psychical Faculties or Processes in the Higher Animals. Science 24, 613-619.

Pech, U., Pooryasin, A., Birman, S., and Fiala, A. (2013). Localization of the contacts between Kenyon cells and aminergic neurons in the Drosophila melanogaster brain using splitGFP reconstitution: splitGFP in Drosophila Mushroom Bodies. Journal of Comparative Neurology 521, 3992-4026.

Pech, U., Revelo, N.H., Seitz, K.J., Rizzoli, S.O., and Fiala, A. (2015). Optical Dissection of Experience-Dependent Pre- and Postsynaptic Plasticity in the Drosophila Brain. Cell Reports 10, 2083-2095.

Perez-Orive, J. (2002). Oscillations and Sparsening of Odor Representations in the Mushroom Body. Science 297, 359-365.

Perisse, E., Burke, C., Huetteroth, W., and Waddell, S. (2013a). Shocking Revelations and Saccharin Sweetness in the Study of Drosophila Olfactory M emory. Current Biology 23, R752-R763.

Perisse, E., Yin, Y., Lin, A.C., Lin, S., Huetteroth, W., and Waddell, S. (2013b). Different Kenyon Cell Populations Drive Learned Approach and Avoidance in Drosophila. Neuron 79, 945-956.

Perisse, E., Owald, D., Barnstedt, O., Talbot, C.B., Huetteroth, W., and Waddell, S. (2016). Aversive Learning and Appetitive Motivation Toggle Feed-Forward Inhibition in the Drosophila Mushroom Body. Neuron 90, 1086-1099.

Pitman, J.L., M cGill, J.J., Keegan, K.P., and Allada, R. (2006). A dynamic role for the mushroom bodies in promoting sleep in Drosophila. Nature 441, 753-756. 
Pitman, J.L., DasGupta, S., Krashes, M.J., Leung, B., Perrat, P.N., and Waddell, S. (2009). There are many ways to train a fly. Fly 3, 3-9.

Plaçais, P.-Y., Trannoy, S., Friedrich, A.B., Tanimoto, H., and Preat, T. (2013). Two Pairs of Mushroom Body Efferent Neurons Are Required for Appetitive Long-Term Memory Retrieval in Drosophila. Cell Reports 5, 769-780.

Poo, C., and Isaacson, J.S. (2009). Odor Representations in Olfactory Cortex: "Sparse" Coding, Global Inhibition, and Oscillations. Neuron 62, 850-861.

Poo, M., Pignatelli, M., Ryan, T.J., Tonegawa, S., Bonhoeffer, T., Martin, K.C., Rudenko, A., Tsai, L.H., Tsien, R.W., Fishell, G., et al. (2016). What is memory? The present state of the engram. BMC Biology 14.

Potter, C.J., Tasic, B., Russler, E.V., Liang, L., and Luo, L. (2010). The Q System: A Repressible Binary System for Transgene Expression, Lineage Tracing, and M osaic Analysis. Cell 141, 536-548.

Ptashne, M. (1988). How eukaryotic transcriptional activators work. Nature 335, 683-689.

Qin, H., Cressy, M., Li, W., Coravos, J.S., Izzi, S.A., and Dubnau, J. (2012). Gamma Neurons Mediate Dopaminergic Input during Aversive Olfactory Memory Formation in Drosophila. Current Biology 22, 608-614.

Quinn, W.G., and Dudai, Y. (1976). Memory phases in Drosophila. Nature 262, 576-577.

Quinn, W.G., Harris, W.A., and Benzer, S. (1974). Conditioned Behavior in Drosophila melanogaster. Proceedings of the National Academy of Sciences 71, 708-712.

Rescorla, R.A. (1967). Pavlovian Conditioning and Its Proper Control Procedures. Psychological Review 74, 71-80.

Rescorla, R.A. (1968). Probability of shock in the presence and absence of cs in fear conditioning. Journal of Comparative and Physiological Psychology 66, 1-5.

Riemensperger, T., Völler, T., Stock, P., Buchner, E., and Fiala, A. (2005). Punishment Prediction by Dopaminergic Neurons in Drosophila. Current Biology 15, 1953-1960.

Roberts, P.D., and Bell, C.C. (2002). Spike timing dependent synaptic plasticity in biological systems. Biological Cybernetics 87, 392-403.

Robertson, H.M., Warr, C.G., and Carlson, J.R. (2003). Molecular evolution of the insect chemoreceptor gene superfamily in Drosophila melanogaster. Proceedings of the National Academy of Sciences 100, 14537-14542.

Rollmann, S.M., Zwarts, L., Edwards, A.C., Yamamoto, A., Callaerts, P., Norga, K., Mackay, T.F.C., and Anholt, R.R.H. (2008). Pleiotropic Effects of Drosophila neuralized on Complex Behaviors and Brain Structure. Genetics 179, 1327-1336.

Root, C.M., M asuyama, K., Green, D.S., Enell, L.E., Nässel, D.R., Lee, C.-H., and Wang, J.W. (2008). A Presynaptic Gain Control Mechanism Fine-Tunes Olfactory Behavior. Neuron 59, 311-321.

Rosenzweig, M. (2005). The Drosophila ortholog of vertebrate TRPA1 regulates thermotaxis. Genes \& Development 19, 419-424. 
Ryner, L.C., Goodwin, S.F., Castrillon, D.H., Anand, A., Villella, A., Baker, B.S., Hall, J.C., Taylor, B.J., and Wasserman, S.A. (1996). Control of Male Sexual Behavior and Sexual Orientation in Drosophila by the fruitless Gene. Cell 87, 1079-1089.

Scaplen, K.M., and Kaun, K.R. (2016). Reward from bugs to bipeds: a comparative approach to understanding how reward circuits function. Journal of Neurogenetics 30, 133-148.

Scheunemann, L., Jost, E., Richlitzki, A., Day, J.P., Sebastian, S., Thum, A.S., Efetova, M., Davies, S.A., and Schwarzel, M. (2012). Consolidated and Labile Odor Memory Are Separately Encoded within the Drosophila Brain. Journal of Neuroscience 32, 17163-17171.

Schnaitmann, C., Garbers, C., Wachtler, T., and Tanimoto, H. (2013). Color Discrimination with Broadband Photoreceptors. Current Biology 23, 2375-2382.

Schneiderman, N., Fuentes, I., and Gormenzano, I. (1961). Acquisition and Extinction of the Classically Conditioned Eyelid Response in the Albino Rabbit. Science 136, 650-652.

Schroll, C., Riemensperger, T., Bucher, D., Ehmer, J., Völler, T., Erbguth, K., Gerber, B., Hendel, T., Nagel, G., Buchner, E., et al. (2006). Light-Induced Activation of Distinct Modulatory Neurons Triggers Appetitive or Aversive Learning in Drosophila Larvae. Current Biology 16, 1741-1747.

Schürmann, F.-W. (1974). Bemerkungen zur Funktion der Corpora pedunculata im Gehirn der Insekten aus morphologischer Sicht. Experimental Brain Research 19, 406-432.

Schwaerzel, M., M onastirioti, M., Scholz, H., Friggi-Grelin, F., Birman, S., and Heisenberg, M. (2003). Dopamine and Octopamine Differentiate between Aversive and Appetitive Olfactory M emories in Drosophila. The Journal of Neuroscience 23, 10495-10502.

Schwartz, J.H., Castellucci, V.F., and Kandel, E.R. (1971). Functioning of identified neurons and synapses in abdominal ganglion of Aplysia in absence of protein synthesis. Journal of Neurophysiology 34, 939-953.

Séjourné, J., Plaçais, P.-Y., Aso, Y., Siwanowicz, I., Trannoy, S., Thoma, V., Tedjakumala, S.R., Rubin, G.M., Tchénio, P., Ito, K., et al. (2011). Mushroom body efferent neurons responsible for aversive olfactory memory retrieval in Drosophila. Nature Neuroscience 14, 903-910.

Semon, R.W. (1904). Die Mneme - Als erhaltendes Prinzip im Wechsel des organischen Geschehens (Leipzig: Wilhelm Engelmann).

Shanbhag, S.R., Iler, B.M., and Steinbrecht, R.A. (1999). Atlas of olfactory organs of Drosophila melanogaster 1 . Types, external organization, innervation and distribution of olfactory sensilla. International Journal of Insect M orphology and Embryology 28, 377-397.

Shaner, N.C., Campbell, R.E., Steinbach, P.A., Giepmans, B.N.G., Palmer, A.E., and Tsien, R.Y. (2004). Improved monomeric red, orange and yellow fluorescent proteins derived from Discosoma sp. red fluorescent protein. Nature Biotechnology 22, 1567-1572.

Shannon, C.E. (1948). A Mathematical Theory of Communication. The Bell System Technical Journal 27, 379-423, 623-656.

Shaw, P.J. (2000). Correlates of Sleep and Waking in Drosophila melanogaster. Science 287, 18341837. 
Shearin, H.K., M acdonald, I.S., Spector, L.P., and Stowers, R.S. (2014). Hexameric GFP and mCherry Reporters for the Drosophila GAL4, Q, and LexA Transcription Systems. Genetics 196, 951-960.

Shih, H.-W., Wu, C.-L., Chang, S.-W., Liu, T.-H., Sih-Yu Lai, J., Fu, T.-F., Fu, C.-C., and Chiang, A.-S. (2015). Parallel circuits control temperature preference in Drosophila during ageing. Nature Communications 6, 7775.

Shimomura, O., Johnson, F.H., and Saiga, Y. (1962). Extraction, Purification and Properties of Aequorin, a Bioluminescent Protein from the Luminous Hydromedusan, Aequorea. Journal of Cellular and Comparative Physiology 59, 223-239.

Siegel, R.W., and Hall, J.C. (1979). Conditioned responses in courtship behavior of normal and mutant Drosophila. Proceedings of the National Academy of Sciences 76, 3430-3434.

Sinakevitch, I., and Strausfeld, N.J. (2006). Comparison of octopamine-like immunoreactivity in the brains of the fruit fly and blow fly. The Journal of Comparative Neurology 494, 460-475.

Sitaraman, D., Zars, M., LaFerriere, H., Chen, Y.-C., Sable-Smith, A., Kitamoto, T., Rottinghaus, G.E., and Zars, T. (2008). Serotonin is necessary for place memory in Drosophila. Proceedings of the National Academy of Sciences 105, 5579-5584.

Sitaraman, D., Aso, Y., Jin, X., Chen, N., Felix, M., Rubin, G.M., and Nitabach, M.N. (2015). Propagation of Homeostatic Sleep Signals by Segregated Synaptic M icrocircuits of the Drosophila Mushroom Body. Current Biology 25, 2915-2927.

Skinner, B.F. (1938). The Behavior of Organisms - An Experimental Analysis (New York: AppletonCentury-Crofts, Inc.).

Skoulakis, E.M.C., Kalderon, D., and Davis, R.L. (1993). Preferential expression in mushroom bodies of the catalytic subunit of protein kinase $A$ and its role in learning and memory. Neuron 11, 197-208.

Spanne, A., and Jörntell, H. (2015). Questioning the role of sparse coding in the brain. Trends in Neurosciences 38, 417-427.

Stettler, D.D., and Axel, R. (2009). Representations of Odor in the Piriform Cortex. Neuron 63, 854-864.

Stocker, R.F. (1994). The organization of the chemosensory system in Drosophila melanogaster: a rewiew. Cell and Tissue Research 275, 3-26.

Stocker, R.F., Lienhard, M.C., Borst, A., and Fischbach, K.F. (1990). Neuronal architecture of the antennal lobe in Drosophila melanogaster. Cell and Tissue Research 262, 9-34.

Stopfer, M., Jayaraman, V., and Laurent, G. (2003). Intensity versus Identity Coding in an Olfactory System. Neuron 39, 991-1004.

Strausfeld, N.J. (1998). Crustacean - Insect Relationships: The Use of Brain Characters to Derive Phylogeny amongst Segmented Invertebrates. Brain, Behavior and Evolution 52, 186-206.

Strausfeld, N.J., Hansen, L., Li, Y., Gomez, R.S., and Ito, K. (1998). Evolution, Discovery, and Interpretations of Arthropod M ushroom Bodies. Learning \& M emory 5, 11-37. 
Strausfeld, N.J., Sinakevitch, I., and Vilinsky, I. (2003). The mushroom bodies of Drosophila melanogaster: An immunocytological and golgi study of Kenyon cell organization in the calyces and lobes. Microscopy Research and Technique 62, 151-169.

Strausfeld, N.J., Sinakevitch, I., Brown, S.M., and Farris, S.M. (2009). Ground plan of the insect mushroom body: Functional and evolutionary implications. The Journal of Comparative Neurology $513,265-291$.

Stuart, G.J., and Spruston, N. (2015). Dendritic integration: 60 years of progress. Nature Neuroscience 18, 1713-1721.

Sweeney, S.T., Broadie, K., Keane, J., Niemann, H., and O'Kane, C.J. (1995). Targeted expression of tetanus toxin light chain in Drosophila specifically eliminates synaptic transmission and causes behavioral defects. Neuron 14, 341-351.

van Swinderen, B., M cCartney, A., Kauffman, S., Flores, K., Agrawal, K., Wagner, J., and Paulk, A. (2009). Shared Visual Attention and M emory Systems in the Drosophila Brain. PLoS ONE 4, e5989.

Takeda, K. (1961). Classical conditioned response in the honey bee. Journal of Insect Physiology 6 , 168-179.

Takemura, S., Aso, Y., Hige, T., Wong, A., Lu, Z., Xu, C.S., Rivlin, P.K., Hess, H., Zhao, T., Parag, T., et al. (2017). A connectome of a learning and memory center in the adult Drosophila brain. ELife 6.

Takeuchi, T., Duszkiewicz, A.J., and Morris, R.G.M. (2014). The synaptic plasticity and memory hypothesis: encoding, storage and persistence. Philosophical Transactions of the Royal Society B: Biological Sciences 369, 20130288.

Tanaka, N.K., Awasaki, T., Shimada, T., and Ito, K. (2004). Integration of Chemosensory Pathways in the Drosophila Second-Order Olfactory Centers. Current Biology 14, 449- 457.

Tanaka, N.K., Tanimoto, H., and Ito, K. (2008). Neuronal assemblies of the Drosophila mushroom body. The Journal of Comparative Neurology 508, 711- 755.

Tang, S., and Guo, A. (2001). Choice Behavior of Drosophila Facing Contradictory Visual Cues. Science 294, 1543-1547.

Tanimoto, H., Heisenberg, M ., and Gerber, B. (2004). Experimental psychology: event timing turns punishment to reward. Nature 430, 983.

Technau, G.M. (1984). Fiber Number in the Mushroom Bodies of Adult Drosophila melanogaster depends on Age, Sex and Experience. Journal of Neurogenetics 1, 113-126.

Tempel, B.L., Bonini, N., Dawson, D.R., and Quinn, W.G. (1983). Reward learning in normal and mutant Drosophila. Proceedings of the National Academy of Sciences 80, 1482-1486.

Tempel, B.L., Livingstone, M.S., and Quinn, W.G. (1984). Mutations in the dopa decarboxylase gene affect learning in Drosophila. Proceedings of the National Academy of Sciences 81, 35773581.

Thompson, R.F. (2005). In Search of Memory Traces. Annual Review of Psychology 56, 1-23.

Thompson, R.F., and Steinmetz, J.E. (2009). The role of the cerebellum in classical conditioning of discrete behavioral responses. Neuroscience 162, 732-755. 
Thorndike, E.L. (1898). Animal Intelligence : An Experimental study of the Associative Processes in Animals (New York: The Macmillan Company).

Thum, A.S., Jenett, A., Ito, K., Heisenberg, M., and Tanimoto, H. (2007). Multiple M emory Traces for Olfactory Reward Learning in Drosophila. Journal of Neuroscience 27, 11132-11138.

Tian, L., Hires, S.A., M ao, T., Huber, D., Chiappe, M.E., Chalasani, S.H., Petreanu, L., Akerboom, J., M cKinney, S.A., Schreiter, E.R., et al. (2009). Imaging neural activity in worms, flies and mice with improved GCaM P calcium indicators. Nature M ethods 6, 875-881.

Tomchik, S.M., and Davis, R.L. (2009). Dynamics of Learning-Related CAMP Signaling and Stimulus Integration in the Drosophila Olfactory Pathway. Neuron 64, 510-521.

Truman, J.W., and Bate, M. (1988). Spatial and temporal patterns of neurogenesis in the central nervous system of Drosophila melanogaster. Developmental Biology 125, 145-157.

Tsien, R.Y. (1998). The Green Fluorescent Protein. Annual Review of Biochemistry 67, 509-544.

Tully, T. (1996). Discovery of genes involved with learning and memory: An experimental synthesis of Hirschian and Benzerian perspectives. Proceedings of the National Academy of Sciences 93, 13460-13467.

Tully, T., and Quinn, W.G. (1985). Classical conditioning and retention in normal and mutant Drosophila melanogaster. Journal of Comparative Physiology A 157, 263-277.

Tully, T., Preat, T., Boynton, S.C., and Del Vecchio, M. (1994). Genetic Dissection of Consolidated Memory in Drosophila. Cell 79, 35-47.

Turner, G.C., Bazhenov, M., and Laurent, G. (2008). Olfactory Representations by Drosophila Mushroom Body Neurons. Journal of Neurophysiology 99, 734-746.

Vaysse, G., and Médoni, J. (1976). Further experiments on the conditioning and pseudoconditioning of the tarsal reflex in the Drosophila (Drosophila melanogaster): effect of low intensity electric shocks. Comptes Rendus Des Seances de La Societe de Biologie et de Ses Filiales 170, 1299- 1304.

Venken, K.J.T., Simpson, J.H., and Bellen, H.J. (2011). Genetic Manipulation of Genes and Cells in the Nervous System of the Fruit Fly. Neuron 72, 202-230.

Vergoz, V., Roussel, E., Sandoz, J.-C., and Giurfa, M. (2007). Aversive Learning in Honeybees Revealed by the Olfactory Conditioning of the Sting Extension Reflex. PLoS ONE 2, e288.

Viswanath, V., Story, G.M., Peier, A.M., Petrus, M.J., Lee, V.M ., Hwang, S.W., Patapoutian, A., and Jegla, T. (2003). Opposite thermosensor in fruitfly and mouse: Ion channels. Nature 423, 822-823.

Vogt, K., Schnaitmann, C., Dylla, K.V., Knapek, S., Aso, Y., Rubin, G.M., and Tanimoto, H. (2014). Shared mushroom body circuits underlie visual and olfactory memories in Drosophila. ELife 3.

Vogt, K., Aso, Y., Hige, T., Knapek, S., Ichinose, T., Friedrich, A.B., Turner, G.C., Rubin, G.M., and Tanimoto, H. (2016). Direct neural pathways convey distinct visual information to Drosophila mushroom bodies. ELife 5 .

Vosshall, L.B., Wong, A.M., and Axel, R. (2000). An Olfactory Sensory Map in the Fly Brain. Cell 102, 147-159. 
Waddell, S. (2013). Reinforcement signalling in Drosophila; dopamine does it all after all. Current Opinion in Neurobiology 23, 324-329.

Waddell, S., Armstrong, J.D., Kitamoto, T., Kaiser, K., and Quinn, W.G. (2000). The amnesiac Gene Product Is Expressed in Two Neurons in the Drosophila Brain that Are Critical for Memory. Cell 103, 805-813.

Wang, J.-H., Ko, G.Y.P., and Kelly, P.T. (1997). Cellular and Molecular Bases of Memory: Synaptic and Neuronal Plasticity. Journal of Clinical Neurophysiology 14, 264-293.

Wang, J.W., Wong, A.M., Flores, J., Vosshall, L.B., and Axel, R. (2003). Two-Photon Calcium Imaging Reveals an Odor-Evoked Map of Activity in the Fly Brain. Cell 112, 271-282.

Wang, Y., M amiya, A., Chiang, A. -S., and Zhong, Y. (2008). Imaging of an Early Memory Trace in the Drosophila M ushroom Body. Journal of Neuroscience 28, 4368-4376.

Ward, J.H. (1963). Hierarchical Grouping to Optimize an Objective Function. Journal of the American Statistical Association 58, 236-244.

Watson, J.B., and Rayner, R. (1920). Conditioned Emotional Reactions. Journal of Experimental Psychology 3, 1-14.

White, B.H., Osterwalder, T.P., Yoon, K.S., Joiner, W.J., Whim, M.D., Kaczmarek, L.K., and Keshishian, H. (2001). Targeted Attenuation of Electrical Activity in Drosophila Using a Genetically Modified K+Channel. Neuron 31, 699-711.

Wilson, R.I., and Laurent, G. (2005). Role of GABAergic Inhibition in Shaping Odor-Evoked Spatiotemporal Patterns in the Drosophila Antennal Lobe. Journal of Neuroscience 25, 90699079.

Wilson, R.I., Turner, G.C., and Laurent, G. (2004). Transformation of Olfactory Representations in the Drosophila Antennal Lobe. Science 303, 366-370.

Wolf, R., Wittig, T., Liu, L., Wustmann, G., Eyding, D., and Heisenberg, M. (1998). Drosophila M ushroom Bodies Are Dispensable for Visual, Tactile, and M otor Learning. Learning \& Memory 5 , 166-178.

Wong, A.M., Wang, J.W., and Axel, R. (2002). Spatial Representation of the Glomerular Map in the Drosophila Protocerebrum. Cell 109, 229-241.

Wright, T.R.F., Beermann, W., Bishop, C.P., Steward, R., Black, B.C., Tomsett, A.D., and Wright, E.Y. (1981). The Genetics of Dopa Decarboxylase in Drosophila melanogaster. Chromosoma 83, 45-58.

Wu, C.-L., Shih, M.-F.M., Lee, P.-T., and Chiang, A.-S. (2013). An Octopamine-Mushroom Body Circuit Modulates the Formation of Anesthesia-Resistant Memory in Drosophila. Current Biology 23, 2346-2354.

Xia, S., Liu, L., Feng, C., and Guo, A. (1997). Memory consolidation in Drosophila operant visual learning. Learning \& M emory 4, 205-218.

Xie, Z, Huang, C., Ci, B., Wang, L., and Zhong, Y. (2013). Requirement of the combination of mushroom body $\gamma$ lobe and $\alpha / \beta$ lobes for the retrieval of both aversive and appetitive early memories in Drosophila. Learning \& Memory 20, 474- 481. 
Xu, T., and Rubin, G.M. (1993). Analysis of genetic mosaics in developing and adult Drosophila tissues. Development 117, 1223-1237.

Yamazaki, D., Hiroi, M., Abe, T., Shimizu, K., M inami-Ohtsubo, M., Maeyama, Y., Horiuchi, J., and Tabata, T. (2018). Two Parallel Pathways Assign Opposing Odor Valences during Drosophila Memory Formation. Cell Reports 22, 2346-2358.

Yang, M.Y., Armstrong, J.D., Vilinsky, I., Strausfeld, N.J., and Kaiser, K. (1995). Subdivision of the Drosophila mushroom bodies by enhancer-trap expression patterns. Neuron 15, 45-54.

Yasuyama, K., M einertzhagen, I.A., and Schürmann, F.-W. (2002). Synaptic organization of the mushroom body calyx in Drosophila melanogaster. Journal of Comparative Neurology 445, 211226.

Yin, J.C.., and Tully, T. (1996). CREB and the formation of long-term memory. Current Opinion in Neurobiology 6, 264-268.

Yin, J.C.., Del Vecchio, M., Zhou, H., and Tully, T. (1995). CREB as a Memory Modulator: induced expression of a dCREB2 activator isoform enhances long-term memory in Drosophila. Cell 81, 107-115.

Yin, J.C.P., Wallach, J.S., Del Vecchio, M., Wilder, E.L., Zhou, H., Quinn, W.G., and Tully, T. (1994). Induction of a dominant negative CREB transgene specifically blocks long-term memory in Drosophila. Cell 79, 49-58.

Yu, D., Baird, G.S., Tsien, R.Y., and Davis, R.L. (2003). Detection of Calcium Transients in Drosophila Mushroom Body Neurons with Camgaroo Reporters. The Journal of Neuroscience 23, 64-72.

Yu, D., Ponomarev, A., and Davis, R.L. (2004). Altered Representation of the Spatial Code for Odors after Olfactory Classical Conditioning: Memory Trace Formation by Synaptic Recruitment. 13.

Yu, D., Keene, A.C., Srivatsan, A., Waddell, S., and Davis, R.L. (2005). Drosophila DPM Neurons Form a Delayed and Branch-Specific M emory Trace after Olfactory Classical Conditioning. Cell 123, 945-957.

Yu, D., Akalal, D.-B.G., and Davis, R.L. (2006). Drosophila $\alpha / \beta$ Mushroom Body Neurons Form a Branch-Specific, Long-Term Cellular Memory Trace after Spaced Olfactory Conditioning. Neuron 52, 845-855.

Yuan, Q., Lin, F., Zheng, X., and Sehgal, A. (2005). Serotonin M odulates Circadian Entrainment in Drosophila. Neuron 47, 115-127.

Zars, T., Fisher, M., Schulz, R., and Heisenberg, M. (2000). Localization of a Short-Term Memory in Drosophila. Science 288, 672-675.

Zhang, S., and Roman, G. (2013). Presynaptic Inhibition of Gamma Lobe Neurons Is Required for Olfactory Learning in Drosophila. Current Biology 23, 2519-2527.

Zhang, J., Campbell, R.E., Ting, A.Y., and Tsien, R.Y. (2002). Creating new fluorescent probes for cell biology. Nature Reviews M olecular Cell Biology 3, 906-918.

Zhang, K., Guo, J.Z., Peng, Y., Xi, W., and Guo, A. (2007a). Dopamine-Mushroom Body Circuit Regulates Saliency-Based Decision-M aking in Drosophila. Science 316, 1901-1904. 
Zhang, W., Ge, W., and Wang, Z. (2007b). A toolbox for light control of Drosophila behaviors through Channelrhodopsin 2-mediated photoactivation of targeted neurons: Control Drosophila behaviors by light. European J ournal of Neuroscience 26, 2405-2416.

Zhou, C., Huang, H., Kim, S.M., Lin, H., M eng, X., Han, K.-A., Chiang, A.-S., Wang, J.W., Jiao, R., and Rao, Y. (2012). Molecular Genetic Analysis of Sexual Rejection: Roles of Octopamine and Its Receptor OAM B in Drosophila Courtship Conditioning. Journal of Neuroscience 32, 14281-14287. 


\section{Acknowledgments}

First and foremost, I want to thank Prof. Dr. André Fiala who gave me the opportunity to work in his lab and provided a lot of help and support. His constant input and knowledge helped me to conduct the experiments. He always had an open ear for problems and helped solving them.

Furthermore, I want to thank my thesis committee members Prof. Dr. Ralf Heinrich and Ph.D. Camin Dean, who gave helpful support and discussions during the thesis committee meeting. Additionally, I thank Ralf Heinrich for reviewing my thesis.

I want to thank the GGNB (Göttingen Graduate School for Neurosciences, Biophysics, and M olecular Biosciences) for their efforts and organization in mounting this doctoral program.

I couldn't have done this work without the help of Dr. Ulrike Pech and Dr. Shubham Dipt, who taught me the calcium imaging procedure and whose imaging and analysis programs I could use. I also thank Dr. David Vasmer for teaching me the MARCM technique, providing me with fly strains, and his scientific input and support.

Special thanks go to Dr. Bart Geurten for the fruitful discussions about the analysis of the data, writing the Matlab scripts, and his inputs regarding science, but also other topics outside the daily lab life.

I further want to thank Dr. Thomas Riemensperger, Dr. Carlotta M artelli, Büşra Çoban, and Clare Hancock for their constant support and intellectual input throughout the Ph.D. project. Especially Jutta Böcker should be mentioned here; her enormous effort in preparing the fly food and every kind of solution, placing orders, and supporting every lab related work; our lab angel, without whom the lab wouldn't run.

I also want to thank Dr. Annekathrin Widman and Clare for reviewing and proof reading of this thesis giving me very helpful suggestions and corrections.

I especially want to mention Haiko Poppinga, who supported me inside the lab, but also outside; and for doing sports and game nights together. Haiko became a special friend to me! 
I also want to thank Büşra for her great hospitality, the endless discussion, hosting game nights, and her friendship. And I don't want to forget Clare, who supported me in the lab, but also has a great humor, which made the time with her always fun.

I also want to thank my students and HiWis - Teresa Lüffe, Annika Hinze, Sophie Siems, Jenifer Rachel, M iriam Berger, and Tim Wellinghof - who supported this work as well.

I want to thank my family, my mother and sister, who are always there for me in every situation of my life. I want to thank my grandmother, who gave me strength, and supported me throughout her and my life, for what I want to dedicate this work to her!

Additionally, I want to thank my girlfriend Jessica Zablotny, who gave me support, especially during writing, and always gives me her affection and love. 


\section{Appendix}

\section{A1 Abbreviations}

(i/o/m)ACT (inner/outer/medial) antennocerebral tract

1-Oct 1-Octanol

3-Oct 3-Octanol

$5 \mathrm{HT} \quad$ serotonin

AC

adenylate cyclase

ACC

activity corrected correlation

$A L$

antennal lobe

ALH

after larval hatching

APF

after pupal formation

APL

anterior paired lateral

ARM

anesthesia resistant memory

ASM

anesthesia sensitive memory

BLA

basolateral complex

BRC

bouton response class

CaM

calmodulin

CAMP

3'5'-cyclic adenosine monophosphate

ChR

channel rhodopsin

$\mathrm{Cl}$

confidence interval

CR

conditioned response

CREB

CAM $P$ response element-binding protein

CS

conditioned stimulus

CXM

cyclohexamide

DA dopamine

DAN dopaminergic neuron

Ddc dopa-decarboxylase

DLG discs large

DNA deoxyribonucleic acid

DPM dorsal paired medial 


\begin{tabular}{|c|c|}
\hline dTRPA1 & Drosophila transient receptor potential A1 \\
\hline FDR & false discovery rate \\
\hline FLP & flippase \\
\hline FRET & fluorescence resonance energy transfer \\
\hline FRT & flippase recognition target \\
\hline GABA & p-aminobutyric acid \\
\hline GFP & green fluorescent protein \\
\hline $\mathrm{GL}$ & glomerulus \\
\hline GRASP & GFP reconstitution across synaptic partners \\
\hline GTP & guanosin-5'-triphosphate \\
\hline HoTX & Hololena toxin \\
\hline hs & heat shock \\
\hline $\mathrm{KC}$ & Kenyon cell \\
\hline lexAop & lexA operator \\
\hline $\mathrm{LH}$ & lateral horn \\
\hline $\mathrm{LN}$ & local inter neuron \\
\hline LTD & long term depression \\
\hline LTM & long term memory \\
\hline LTP & long term potentiation \\
\hline MARCM & mosaic analysis with a repressible cell marker \\
\hline MB & mushroom body \\
\hline MBNbs & MB neuroblasts \\
\hline MBON & MB output neuron \\
\hline $\mathrm{MCH}$ & 4-Methylcyclohexanol \\
\hline M Oil & mineral oil \\
\hline M TM & middle term memory \\
\hline NPY & neuropeptide $Y$ \\
\hline NS & neutral stimulus \\
\hline $\mathrm{OA}$ & octopamine \\
\hline OR & odorant receptor \\
\hline ORCO & OR co-receptor \\
\hline OSN & olfactory sensory neurons \\
\hline PAM & protocerebral anterior medial \\
\hline PBS & phosphate buffered saline \\
\hline PBST & PBS + Triton X 100 \\
\hline
\end{tabular}


Apendix

$\begin{array}{ll}\text { PER } & \text { proboscis extension reflex } \\ \text { PFA } & \text { paraformaldehyde } \\ \text { PKA } & \text { protein kinase A } \\ \text { PLTX } & \text { Plectreurys toxin } \\ \text { PN } & \text { projection neurons } \\ \text { PPL } & \text { protocerebral posterior lateral } \\ \text { PTX } & \text { pertussis toxin } \\ \text { QUAS } & \text { QF upstream activating sequence } \\ \text { RFP } & \text { red fluorescent protein } \\ \text { rUt } & \text { rutabaga } \\ \text { SEM } & \text { standard error of the mean } \\ \text { SER } & \text { sting extension reflex } \\ \text { Shit } & \text { Shibire temperature-sensitive } \\ \text { SNPF } & \text { short neuropeptide F } \\ \text { SPGFP } & \text { split GFP } \\ \text { STD } & \text { standard deviation } \\ \text { STM } & \text { short term memory } \\ \text { syb } & \text { synaptobrevin } \\ \text { Syp } & \text { synaptophysin } \\ \text { TeTxLC } & \text { tetanus toxin light chain } \\ \text { TH } & \text { tyrosine hydroxylase } \\ \text { UAS } & \text { upstream activating sequence } \\ \text { UR } & \text { unconditioned response } \\ \text { US } & \end{array}$




\section{- Florian Bilz -}

\section{Personal Details}

Home address:

Auf der Lehmbünde 21,

37085 Göttingen

E-mail:

florian.bilz@gmx.de

Family status:

unmarried

Nationality:

German

Birthday/ -place:

07. August 1986, Wismar

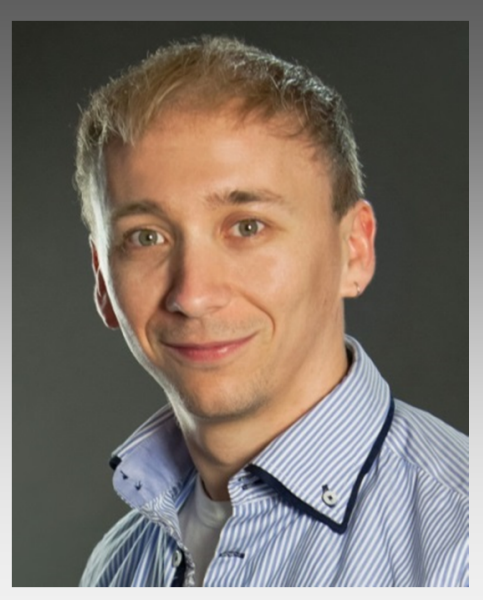

\section{Academic Education}

Feb. 2015 - Jul. 2018

Oct. 2011 - Mar. 2014

Oct. 2008 - Sep. 2011

\section{Trainings}

Jun. 2013 - Sep. 2013
Ph.D. student in Neuroscience, Georg-August-Universität Göttingen Thesis: "Optical Analysis of Synaptic Plasticity Underlying Associative Learning in Drosophila melanogaster"

Master program in Biology with focus on Neurobiology and Behavior, Freie Universität Berlin

Thesis: "Coding and Interaction of Sex and Food Related Odors in the Drosophila Antennal Lobe". Degree: Master of Science

Bachelor program in Biological Science, Universität Rostock Thesis: "Untersuchungen zur Rolle von Connexin 31 und Connexin 43 während der Proliferation und Differenzierung humaner neuronaler Progenitorzellen". Degree: Bachelor of Science

Summer Scholarship, autonomic development and implementation of the project "Aversive Training Changes Odor Response" in mice, Stowers Institute for M edical Research,

AG Dr. Ron Yu, 1000 E 50th Street, Kansas City, M issouri 64110, USA

\section{Awards}

Félix Dujardin Award 2018 for the best poster presentation. Biennial Conference of the Arthropod Neuroscience Network 2018. Altleinigen, 29th March, 2018 


\section{Publications}

\section{Paper:}

2018

(submitted)

2015

\section{Book chapter}

2017

Posters:

2018

2017

2015

2014

2013
Bilz F., Geurten B.R.H., Fiala A. (submitted). Visualization of a distributed synaptic memory code in the Drosophila brain. Science Advances.

Lebreton S., Trona F., Borrero-Echeverry F., Bilz F., Grabe V., Becher P.G., Carlsson M.A., Nässel D.R., Hansson B.S., Sachse S., Witzgall P. 2015. Feeding regulates sex pheromone attraction and courtship in Drosophila females. Scientific Reports 5: 13132.

Riemensperger T., Bilz F., Fiala A. (2017). Visualization of Synapses and Synaptic Plasticity in the Drosophila Brain. Decoding Neural Circuit Structure and Function (Çelik A. and Wernet M.F. (eds), Springer International Publishing AG 2017).

F. Bilz, B. Geurten, A. Fiala. Optical Analysis of Synaptic Plasticity Underlying Associative Learning in Drosophila melanogaster. Arthropod Neuroscience Network Meeting 2018, Altleiningen, 27th29th March, 2018

F. Bilz, B. Geurten, A. Fiala. Optical Analysis of Synaptic Plasticity Underlying Associative Learning in Drosophila melanogaster. 12th Göttingen Meeting of the German Neuroscience Society, Göttingen, 22nd-25th March, 2017

F. Bilz, S. Nagel, H.-J. Pflüger \& M. Schubert. Optophysiological investigation on the integral role of octopaminergic neurons in the motor neuron system of Drosophila melanogaster. 11th Göttingen Meeting of the German Neuroscience Society, Göttingen, 18th-21th March, 2015

H.-J. Pflüger, F. Bilz, M. Schubert, K. Lehmann, S. Hartfil, N. L. Kononenko. Tyraminergic/ octopaminergic neurons and some of their functions in locusts and fruitflies. 11th International Congress of Neuroethology (ICN), Sapporo, Hokkaido, Japan, 28th July - 1st August, 2014

M. Schubert, F. Bilz, V. Antemann \& H.-J. Pflüger. Anatomical and in vivo optical imaging analysis of metathoracic DUM neurons in the locust, Schistocerca gregaria. Proc. of the 34th Göttingen Neurobiology Conference, Göttingen, 13th-16th March, 2013 